LAWRENCE LIVERMORE N A T IO N A L LABORATORY
Weighted Essentially Non-Oscillatory Simulations and Modeling of Complex Hydrodynamic Flows. Part 2. Single-Mode Richtmyer-Meshkov Instability with Reshock

O. Schilling, M. Latini

November 1, 2004 
This document was prepared as an account of work sponsored by an agency of the United States Government. Neither the United States Government nor the University of California nor any of their employees, makes any warranty, express or implied, or assumes any legal liability or responsibility for the accuracy, completeness, or usefulness of any information, apparatus, product, or process disclosed, or represents that its use would not infringe privately owned rights. Reference herein to any specific commercial product, process, or service by trade name, trademark, manufacturer, or otherwise, does not necessarily constitute or imply its endorsement, recommendation, or favoring by the United States Government or the University of California. The views and opinions of authors expressed herein do not necessarily state or reflect those of the United States Government or the University of California, and shall not be used for advertising or product endorsement purposes.

This work was performed under the auspices of the U.S. Department of Energy by University of California, Lawrence Livermore National Laboratory under Contract W-7405-Eng-48. 


\title{
Weighted Essentially Non-Oscillatory Simulations and Modeling of Complex Hydrodynamic Flows. Part 2. Single-Mode Richtmyer-Meshkov Instability with Reshock
}

\author{
Marco Latini* $\quad$ Oleg Schilling ${ }^{\dagger}$
}

June 30, 2004

\begin{abstract}
The Richtmyer-Meshkov instability is a fundamental fluid instability that occurs when perturbations on an interface separating gases with different properties grow following the passage of a shock. This instability is typically studied in shock tube experiments, and constitutes a fundamental example of a complex hydrodynamic flow. Numerical simulations and models for the instability growth and evolution have also been used to further understand the physics of the Richtmyer-Meshkov instability. In the present work, the formally high-order accurate weighted essentially non-oscillatory (WENO) shock-capturing method using a third-order total-variation diminishing (TVD) Runge-Kutta time-evolution scheme (as implemented in the HOPE code [57]) is applied to simulate the single-mode RichtmyerMeshkov instability with reshock in two spatial dimensions. The initial conditions and computational domain for the simulations are modeled after the Collins and Jacobs [23] single-mode, Mach 1.21 air(acetone) $/ \mathrm{SF}_{6}$ shock tube experiment. The following boundary conditions are used: (1) periodic in the spanwise direction corresponding to the cross-section of the test section; (2) outflow at the entrance of the test section in the streamwise direction, and; (3) reflecting at the end wall of the test section in the streamwise direction. The present investigation has three principal motivations: (1) to provide additional validation of the HOPE code against available experimental data; (2) to provide numerical simulation data for detailed analysis of mixing induced by the Richtmyer-Meshkov instability with reshock, and; (3) to systematically investigate the dependence of mixing properties on both the order of WENO reconstruction and spatial resolution. The present study constitutes the first comprehensive application of the high-resolution WENO method to the Richtmyer-Meshkov instability with reshock, as well as analysis of the resulting mixing.

First, analytical, semi-analytical, and phenomenological models for the growth of a single- and multi-mode perturbation are reviewed (impulsive, vortex, perturbation, potential flow, and asymptotic power-law growth models), including models for diffuse and reshocked interfaces. A model for baroclinic circulation deposition is also reviewed. $\mathrm{Nu}-$ merical simulations are performed using the third-, fifth-, and ninth-order WENO method with spatial resolutions corresponding to a uniform grid with 128, 256, and 512 points per initial perturbation wavelength. The density from the fifth- and ninth-order simulation
\end{abstract}

\footnotetext{
${ }^{*}$ Applied and Computational Mathematics, California Institute of Technology, Pasadena, CA 91125. E-mail: mlatini@acm.caltech.edu

${ }^{\dagger}$ University of California, Lawrence Livermore National Laboratory, Livermore, CA 94551. E-mail: schilling1@llnl.gov
} 
is compared to the corrected experimental PLIF images of Collins and Jacobs at selected times. The amplitude obtained from the fifth-order simulation at a resolution of 256 points per initial perturbation wavelength is compared to the experimental data of Collins and Jacobs and to the predictions of linear and nonlinear amplitude growth models before and after reshock. The prediction of the Zhang-Sohn nonlinear amplitude growth model is in best agreement with the simulation data prior to reshock. The simulation data is also in excellent agreement with the experimentally-measured amplitude prior to reshock. The absence of the initial rarefaction wave (resulting from the rupture of the membrane that generates the first shock in the experiment) in the numerical simulations results in a time lag between the numerical and experimental interface evolution following reshock. The results of this component of the present investigation also serve as an additional validation of the HOPE code as applied to a shock-induced hydrodynamic instability.

Second, local and global properties of the mixing during the linear, nonlinear, pre- and post-reshock, and late-time phases are investigated and discussed, including a quantitative investigation of the time-dependence and structure of various related mixing parameters defined in terms of the mole fraction and one-dimensional energy spectra. Spatial averaging of quantities along the spanwise (periodic) flow direction yields streamwise profiles, and is used to define instantaneous Reynolds and Favre averages and fluctuations. The fluctuations are Fourier-transformed along the spanwise direction to define time-dependent energy spectra as a function of the one-dimensional wavenumber. Global statistics are obtained by integrating these spectra over all wavenumbers. Several time-dependent volume-averaged quantities are also considered. The effects of reshock on these quantities are examined and discussed in detail. A comparison of simulations with reflecting and outflow boundary conditions at the end wall of the test section exhibits the effects of additional reflected wave interactions following reshock on the above quantities. The simulations are carried out to much longer times $(t=18 \mathrm{~ms})$ than reported in the experiment $(t=11 \mathrm{~ms})$ to study the evolution of profiles, spectra, and statistics in the decay and quasi-decay regimes (corresponding to outflow and reflecting boundary conditions, respectively) - quantities that are not currently experimentally-measurable.

Third, the dependence of the mixing layer width, mixing properties, spectra, and statistics on the grid resolution and order of WENO spatial flux reconstruction is comprehensively investigated. Simulations with varying orders of reconstruction and grid resolutions have different characteristics of intrinsic numerical diffusion arising from the truncation and other numerical errors in the algorithm. Quantities such as mixing fractions and energy spectra are sensitive to the numerical diffusion prior to reshock: it is shown that these sensitivities are significantly amplified following reshock when the energy deposited by the shock on the evolving interface induces the formation of small-scale features and amplifies the fluctuations of all quantities within the mixing layer. It is shown that simulations on coarse grids and using low orders of WENO reconstruction preserve large-scale structures and symmetry even at late times, while simulations on fine grids and using higher orders of reconstruction exhibit fragmentation of the structures, breaking of symmetry, and increased mixing. The differences between the simulations can be quantified by the time-evolution of the fluctuating kinetic energy and enstrophy, as well as the density and pressure variance. The investigation suggests that similar flow features are qualitatively and quantitatively captured by either increasing the spatial resolution or the order of reconstruction. The computational scaling shows that increasing the order of reconstruction from third to fifth or from fifth to ninth approximately doubles the computational cost. However, for a given order of reconstruction, doubling the spatial resolution from 128 to 256 or from 256 to 512 points per initial perturbation wavelength incurs a five- to eight-fold increase in computational cost.

Based on the results of the present investigation, it is concluded that the WENO method using high order reconstruction is well-suited for the simulation and analysis of complex hydrodynamic flows induced by shock-interface interactions. 


\section{Contents}

1 Introduction $\quad \mathbf{1 5}$

1.1 Previous single-mode Richtmyer-Meshkov instability experiments with reshock 17

1.2 Previous numerical simulations of single-mode Richtmyer-Meshkov instability with reshock ............................ 17

1.3 Objectives of the current investigation . . . . . . . . . . . 20

2 Models for the perturbation amplitude and mixing layer growth and for circulation deposition $\quad 23$

2.1 Impulsive models . . . . . . . . . . . . . . . . . . . . . . . . 23

2.1.1 The Richtmyer model . . . . . . . . . . . . . . . . . . . 24

2.1.2 The Meyer-Blewett model . . . . . . . . . . . . . . . . 25

2.1.3 The Fraley perturbation solution . . . . . . . . . . . . . . . 25

2.1.4 The Vandenboomgaerde et al. model . . . . . . . . . . . . . 26

2.1.5 The Brouillette-Sturtevant model for diffuse interfaces . . . . . . . . . . 27

2.2 The Jacobs-Sheeley vortex model . . . . . . . . . . . . . . . . . . . 28

2.3 Perturbation models . . . . . . . . . . . . . . . . . . . . . . . 29

2.3.1 The Zhang-Sohn model . . . . . . . . . . . . . . . . . . . 29

2.3.2 The Vandenboomgaerde et al. model . . . . . . . . . . . . . . . . 32

2.4 Potential flow models . . . . . . . . . . . . . . . . . . . . . . . 33

2.4.1 The Layzer model for the Rayleigh-Taylor instability . . . . . . . . . . . 33

2.4.2 The Hecht-Alon-Shvarts model for the Richtmyer-Meshkov instability . 35

2.4.3 The Mikaelian model for arbitrary initial perturbations . . . . . . . . 35

2.4.4 The Zhang model for the velocity of spikes . . . . . . . . . . . 36

2.4.5 The Goncharov model for arbitrary Atwood numbers . . . . . . . . . . . 37

2.4 .6 The Sohn model . . . . . . . . . . . . . . . . . . . 37

2.4.7 The Sadot et al. empirical model for the Richtmyer-Meshkov instability 38

2.5 Power-law models for multi-mode initial perturbations and following reshock . 38

2.5.1 The Alon et al. bubble merger model for $A=1 \ldots$. . . . . . . . . . 39

2.5.2 The Rikanati et al. vortex model for bubble merger when $A \rightarrow 0 \quad \ldots \quad 40$

2.5.3 The Prasad et al. late-time model . . . . . . . . . . . . . . . 41

2.5.4 The Youngs model for multi-mode initial conditions . . . . . . . . . . 42

2.5.5 The Mikaelian model for a reshocked interface . . . . . . . . . . . . 43

2.5.6 The Brouillette-Sturtevant model for reshocked interfaces . . . . . . . . 43

2.6 The Samtaney-Zabusky-Ray model for baroclinic circulation deposition . . . . 44

3 Numerical simulations of the single-mode Richtmyer-Meshkov instability $\begin{array}{ll}\text { with reshock } & 47\end{array}$

3.1 Numerical simulation parameters . . . . . . . . . . . . . . . . . . . . . 48

3.1 .1 Initial gas composition . . . . . . . . . . . . . . . . . 48

3.1.2 Computational domain and interfacial perturbation . . . . . . . . . 49

3.2 Qualitative comparison of instability evolution to experimental PLIF images . . 49

3.3 Evolution of the amplitude prior to reshock . . . . . . . . . . . . 52

3.3.1 Numerical determination of the mixing layer width . . . . . . . . . . 52

3.3.2 Quantitative comparison to experimental data and simulated Schlieren images . . . . . . . . . . . . . . . . . . 5 5 53 
3.3.3 Comparison to the predictions of impulsive models . . . . . . . . . . 56

3.3.4 Comparison to the predictions of the Jacobs-Sheeley vortex model . . . 56

3.3.5 Comparison to the predictions of perturbation models . . . . . . . 58

3.4 Evolution of the mixing layer amplitude following reshock and comparison to reshock models . . . . . . . . . . . . . . . . . . 58

3.5 Circulation deposition on the interface . . . . . . . . . . . . . . 63

4 Local and global analysis of mixing in two-dimensional, single-mode Richtmyer$\begin{array}{ll}\text { Meshkov instability with reshock } & 65\end{array}$

4.1 Time-evolution of mole, volume fraction, and mixing fraction profiles . . . . . . 65

4.1.1 Evolution of the mole fraction profile $\langle X\rangle \ldots \ldots 6$

4.1.2 Evolution of the profiles of the averaged chemical product from a fast kinetic reaction $\left\langle X_{p}\right\rangle, X_{p}(\langle X\rangle)$ and of the mixing fraction $\xi \ldots 68$

4.1.3 Evolution of the profiles of the volume fractions $\left\langle f_{1}\right\rangle,\left\langle f_{2}\right\rangle$ and of the mixing fractions $\xi$ and $\theta \ldots \ldots \ldots 70$

4.2 Time-evolution of mixing fractions and of volume-averaged quantities . . . . 70

4.2.1 Evolution of $P_{t}, P_{m}, \Theta$, and $\Xi \ldots \ldots \ldots \ldots$

4.2.2 Evolution of the volume-averaged velocity components and pressure . . 73

4.3 Time-evolution of the fluctuating kinetic energy, fluctuating enstrophy, density variance, and pressure variance spectra . . . . . . . . . . . . . . 73

4.4 Time-evolution of statistics . . . . . . . . . . . . . . . . . 81

4.5 Comparison of mixing quantities at selected times . . . . . . . . . . 84

4.5.1 Comparison of mixing quantities at early and late times . . . . . . 85

4.5.2 Comparison of mixing quantities before and after reshock . . . . . . 88

4.6 The effects of reflected waves: outflow and reflecting boundary conditions . . . 88

4.6.1 Comparison of mixing layer width, mixing fractions, and profiles . . . . 93

4.6 .2 Comparison of statistics . . . . . . . . . . . . . . . . . 93

5 The effects of spatial resolution and order of spatial reconstruction on mix$\begin{array}{ll}\text { ing } & \mathbf{9 7}\end{array}$

5.1 Grid resolutions and orders of reconstruction . . . . . . . . . . . . . . 98

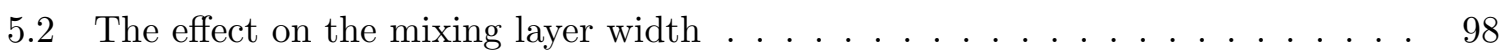

5.3 The effect on mixing profiles . . . . . . . . . . . . . . . . . . . . 99

5.4 The effect on mixing fractions . . . . . . . . . . . . . . . . . . 102

5.5 The effect on spectra . . . . . . . . . . . . . . . . . 108

5.6 The effect on statistics . . . . . . . . . . . . . . . . . . . . . 118

5.7 Temporal progression of density and vorticity . . . . . . . . . . . . . . . 120

5.8 Computational scaling of the simulations . . . . . . . . . . . . . . . . . 140

6 Summary and conclusions $\quad 141$

6.1 Mixing layer growth . . . . . . . . . . . . . . . . . . . . . 143

6.1.1 Comparison to linear and nonlinear models prior to reshock . . . . . . . 143

6.1.2 Comparison to reshock models . . . . . . . . . . . . . . . . . 143

6.1.3 The effects of outflow and reflecting boundary conditions . . . . . . . . 144

6.2 Local and global mixing analysis . . . . . . . . . . . . . . . . . 144

6.2.1 Profiles, mixing fractions, spectra, and statistics . . . . . . . . . 144

6.2 .2 The effects of reshock . . . . . . . . . . . . . . . . . 145 
6.2.3 The effects of outflow and reflecting boundary conditions . . . . . . . 145

6.3 The effects of order of reconstruction and grid resolution . . . . . . . . . . . 146

6.4 Conclusions . . . . . . . . . . . . . . . . . . . . . 147 


\section{List of Figures}

1 The single-mode Richtmyer-Meshkov instability prior to reshock . . . . . . . 18

2 The single-mode Richtmyer-Meshkov instability during reshock . . . . . . . . . 19

3 The single-mode Richtmyer-Meshkov instability following reshock . . . . . . . . 19

4 Schematic of the bubble and spike amplitudes $a_{b}$ and $a_{s}$ and the mixing layer width $h \ldots \ldots \ldots \ldots \ldots \ldots \ldots \ldots$

5 Oblique shock refraction with reflected shock wave . . . . . . . . . . 46

6 Comparison of Collins and Jacobs experimental PLIF images and numerical simulation results for the evolution of the Richtmyer-Meshkov instability . . . .

7 Further comparison of Collins and Jacobs experimental PLIF images and numerical simulation results for the evolution of the Richtmyer-Meshkov instability 51

$8 x$ - $t$ diagram from the simulation of the Richtmyer-Meshkov instability . . . . 54

9 Comparison of experimental measurements and numerical data for the mixing layer width in the case of a $M a=1.21$ shock . . . . . . . . . . . . . 55

10 Simulated density Schlieren images of the Richtmyer-Meshkov instability with

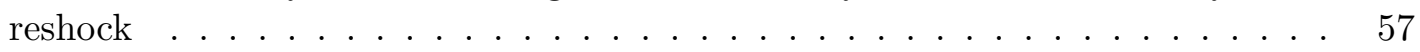

11 Linear models for the mixing layer amplitude . . . . . . . . . . . . . . . . . 59

12 Jacobs-Sheeley vortex model for the mixing layer amplitude . . . . . . . . . . . . 59

13 Nonlinear perturbation series models for mixing layer amplitude . . . . . . . . 60

14 Nonlinear Padé models for mixing layer amplitude . . . . . . . . . . . . . . . 61

15 Ratio of amplitude from models to the simulation data . . . . . . . . . . . . 62

16 Mixing layer width for reshocked interface with comparison to the Mikaelian and Brouillette and Sturtevant models . . . . . . . . . . . . . . . . . 62

17 Circulation deposition . . . . . . . . . . . . . . . . . . . 64

18 Time-evolution of $\langle X\rangle$ with $x$-axis recentered and $\langle X\rangle$ with $x$-axis recentered

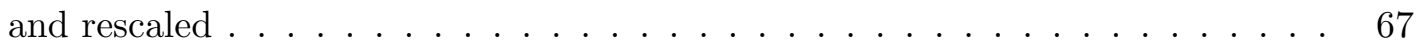

19 Time-evolution of $\left\langle X_{p}\right\rangle$ and $X_{p}\langle X\rangle$ recentered and rescaled . . . . . . . . . 69

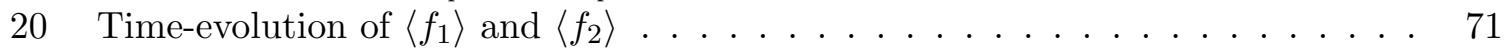

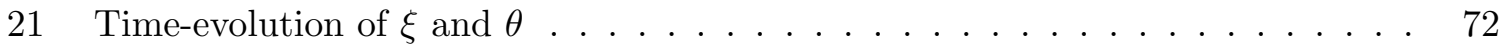

22 Time-evolution of $h(t), W(t), P_{t}, P_{m}, \Theta, \Xi,\langle u\rangle_{x y},\langle v\rangle_{x y}$, and $\langle p\rangle_{x y} \ldots \ldots .74$

23 Time-evolution of the streamwise and spanwise fluctuating kinetic energy spectra $E_{u^{\prime \prime} u^{\prime \prime}}(k, t)$ and $E_{v^{\prime \prime} v^{\prime \prime}}(k, t) \ldots \ldots \ldots \ldots \ldots$. . . . . . . . . 76

24 Time-evolution of the fluctuating kinetic energy spectrum $E(k, t)$ and fluctuating enstrophy spectrum $E_{\omega^{\prime \prime} \omega^{\prime \prime}}(k, t) \ldots \ldots \ldots \ldots \ldots$

25 Time-evolution of the ratio of the streamwise and spanwise fluctuating kinetic energy spectra $E_{u^{\prime \prime} u^{\prime \prime}}(k, t) / E_{v^{\prime \prime} v^{\prime \prime}}(k, t) \ldots \ldots \ldots \ldots$. . . . . . . . . .

26 Time-evolution of the density variance spectrum $E_{\rho^{\prime} \rho^{\prime}}(k, t)$ and the pressure variance spectrum $E_{p^{\prime} p^{\prime}}(k, t) \ldots \ldots \ldots \ldots \ldots$

27 Time-evolution of fluctuating kinetic energy $E(t)$ and its components, fluctuating enstrophy $E_{\omega^{\prime \prime} \omega^{\prime \prime}}(t)$, density variance $E_{\rho^{\prime} \rho^{\prime}}(t)$, and pressure variance $E_{p^{\prime} p^{\prime}}(t)$

28 Time-evolution of the ratio of the streamwise and spanwise fluctuating kinetic energy $E_{u^{\prime \prime} u^{\prime \prime}}(t) / E_{v^{\prime \prime} v^{\prime \prime}}(t) \ldots \ldots \ldots \ldots \ldots$

29 Profiles of $\langle X\rangle,\left\langle X_{p}\right\rangle, X_{p}(\langle X\rangle), \xi,\left\langle f_{1}\right\rangle,\left\langle f_{2}\right\rangle, \theta$ and fluctuating kinetic energy spectrum $E(k, t)$ at $t=6 \mathrm{~ms}$ and $t=7 \mathrm{~ms} \ldots \ldots \ldots \ldots$

30 Profiles of $\langle X\rangle,\left\langle X_{p}\right\rangle, X_{p}(\langle X\rangle), \xi,\left\langle f_{1}\right\rangle,\left\langle f_{2}\right\rangle, \theta$ and fluctuating kinetic energy spectrum $E(k, t)$ at $t=12 \mathrm{~ms}$ and $t=18 \mathrm{~ms} \ldots \ldots \ldots$ 
31 Comparison of $\langle X\rangle$ centered and not centered, $\left\langle X_{p}\right\rangle$, and $X_{p}(\langle X\rangle)$ at $t=6.38$ $\mathrm{ms}$ and $t=6.68 \mathrm{~ms} \ldots \ldots \ldots \ldots \ldots \ldots \ldots \ldots$

32 Comparison of $\left\langle f_{1}\right\rangle$ and $\left\langle f_{2}\right\rangle$ and $\theta$ at $t=6.38$ and $t=6.68 \mathrm{~ms} \ldots \ldots$

33 Comparison of the fluctuating kinetic energy spectrum $E(k, t)$, fluctuating enstrophy spectrum $E_{\omega^{\prime \prime} \omega^{\prime \prime}}(k, t)$, density variance spectrum $E_{\rho^{\prime} \rho^{\prime}}(k, t)$, and pressure variance spectrum $E_{p^{\prime} p^{\prime}}(k, t)$ at $t=6.38 \mathrm{~ms}$ and $t=6.68 \mathrm{~ms} \ldots \ldots$

34 Ratios of $E(k, t), E_{\omega^{\prime \prime} \omega^{\prime \prime}}(k, t), E_{\rho^{\prime} \rho^{\prime}}(k, t)$, and $E_{p^{\prime} p^{\prime}}(k, t)$ before and after reshock 91

35 Time-evolution of $h(t), W(t), P_{m}, P_{t}, \Theta, \Xi,\langle u\rangle_{x y},\langle v\rangle_{x y}$, and $\langle p\rangle_{x y}$ for outflow and reflecting boundary conditions . . . . . . . . . . . .

36 Time-evolution of fluctuating kinetic energy $E(t)$, fluctuating enstrophy $E_{\omega^{\prime \prime} \omega^{\prime \prime}}(t)$, density variance $E_{\rho^{\prime} \rho^{\prime}}(t)$, and pressure variance $E_{p^{\prime} p^{\prime}}(t)$ for outflow and reflecting

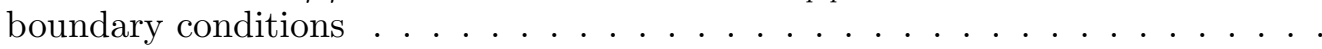

37 Comparison of mixing layer width $h(t)$ when the order of reconstruction and grid resolution are varied . . . . . . . . . . . . . . 100

38 Comparison of the mole fraction profile $\langle X\rangle$ at $t=6,7,12,18$ ms when the order of reconstruction and grid resolution are varied . . . . . . . . . . . . . 101

39 Comparison of the product mole fraction profile $\left\langle X_{p}\right\rangle$ at $t=6,7,12,18$ ms when the order of reconstruction and the grid resolution are varied . . . . . . . 103

40 Comparison of $X_{p}(\langle X\rangle)$ at $t=6,7,12,18 \mathrm{~ms}$ when the order of reconstruction and the grid resolution are varied . . . . . . . . . . . . . 104

41 Comparison of $\theta$ at $t=6,7,12,18 \mathrm{~ms}$ when the order of reconstruction and grid resolution are varied . . . . . . . . . . . . . . . 105

42 Comparison of $\xi$ at $t=6,7,12,18 \mathrm{~ms}$ when the order of reconstruction and the grid resolution are varied . . . . . . . . . . . . 106

43 Comparison of the mixing fractions $P_{t}, P_{m}, \Xi$, and $\Theta$ when the order of reconstruction and grid resolution are varied . . . . . . . . . . . . . 107

44 Comparison of the fluctuating kinetic energy spectrum $E(k, t)$ at $t=6,7,12,18$ $\mathrm{ms}$ when the order of reconstruction and the grid resolutions are varied . . . . 109

45 Ratio of the fluctuating kinetic energy spectrum from the simulations to the spectrum from the ninth-order simulation on the fine grid at $t=6,7,12,18 \mathrm{~ms}$ when the order of reconstruction and grid resolution are varied . . . . . . .

46 Comparison of the fluctuating enstrophy spectrum $E_{\omega^{\prime \prime} \omega^{\prime \prime},}(k, t)$ at $t=6,7,12,18$ ms when the order of reconstruction and grid resolutions are varied . . . . . . . 111

47 Ratio of the fluctuating enstrophy spectrum from the simulations to the spectrum from the ninth-order simulation on the fine grid at $t=6,7,12,18 \mathrm{~ms}$ when the order of reconstruction and grid resolution are varied $\ldots \ldots \ldots$

48 Comparison of the density variance spectrum $E_{\rho^{\prime} \rho^{\prime}}(k, t)$ at $t=6,7,12,18 \mathrm{~ms}$ when the order of reconstruction and the grid resolutions are varied . . . . . 114

49 Ratio of the density variance spectrum from the simulations to the spectrum from the ninth-order simulation on the fine grid at $t=6,7,12,18 \mathrm{~ms}$ when the order of reconstruction and grid resolution are varied . . . . . . . . . .

50 Comparison of the pressure variance spectrum $E_{p^{\prime} p^{\prime}}(k, t)$ at $t=6,7,12,18 \mathrm{~ms}$ when the order of reconstruction and the grid resolutions are varied . . . . 116

51 Ratio of the pressure variance spectrum from the simulations to the spectrum from the ninth-order simulation on the fine grid at $t=6,7,12,18 \mathrm{~ms}$ when the order of reconstruction and grid resolution are varied . . . . . . . . . . . 117 
52 Comparison of $E_{u^{\prime \prime} u^{\prime \prime}}(t), E_{v^{\prime \prime} v^{\prime \prime}}(t), E(t), E_{\omega^{\prime \prime} \omega^{\prime \prime}}(t), E_{\rho^{\prime} \rho^{\prime}}(t)$, and $E_{p^{\prime} p^{\prime}}(t)$ when the order of reconstruction and grid resolution are varied . . . . . . . . . . 119

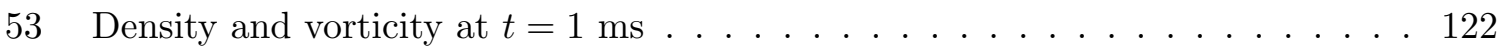

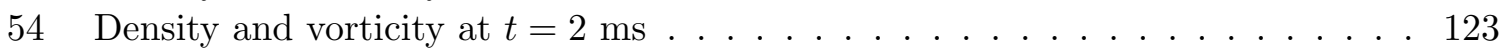

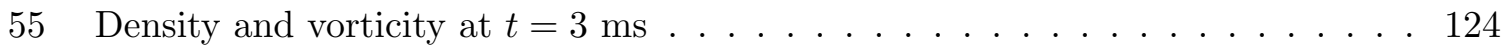

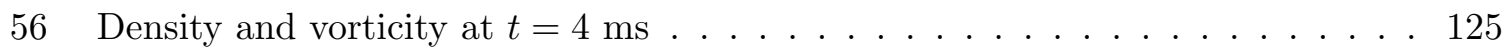

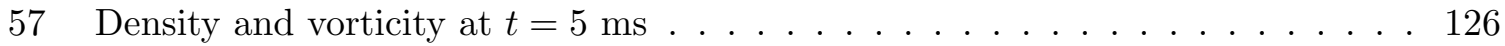

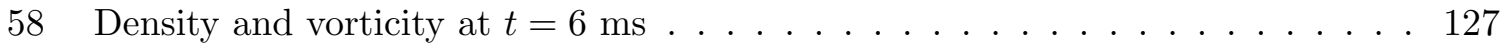

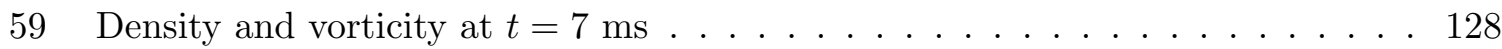

60 Density and vorticity at $t=8 \mathrm{~ms} \ldots \ldots \ldots \ldots \ldots \ldots \ldots$

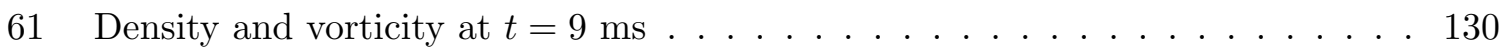

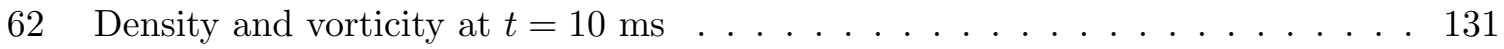

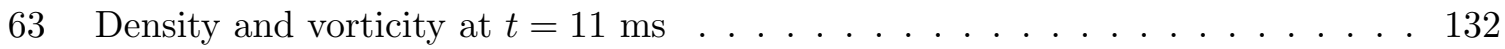

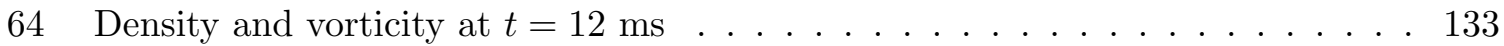

65 Density and vorticity at $t=13 \mathrm{~ms} \ldots \ldots \ldots \ldots \ldots \ldots \ldots$

66 Density and vorticity at $t=14 \mathrm{~ms} \ldots \ldots \ldots \ldots \ldots \ldots$

67 Density and vorticity at $t=15 \mathrm{~ms} \ldots \ldots \ldots \ldots \ldots \ldots$

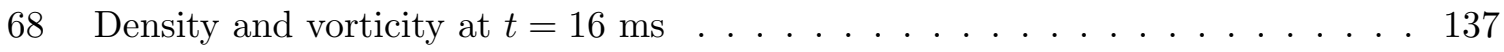

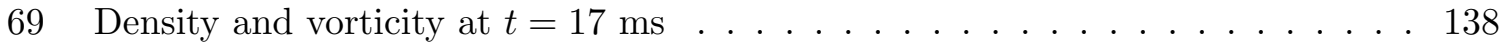

70 Density and vorticity at $t=18 \mathrm{~ms} \ldots \ldots \ldots \ldots \ldots \ldots$ 


\section{List of Tables}

1 Pre- and post-shock values obtained in the simulation . . . . . . . . . 54

2 Grid resolutions for the simulations . . . . . . . . . . . . . . . 98

3 Ratio of CPU times for advancing the simulations by $\Delta t=0.1 \mathrm{~ms}$ compared with the time for the fifth-order medium grid simulation . . . . . . . . . . . 140 


\section{List of Symbols}

$a(t) \quad$ mixing layer amplitude, defined in Eq. (6), page 23

$a_{0}^{-(+)}$

pre-shock (post-shock) initial perturbation amplitude, page 24

$a_{1}^{-(+)}$

pre-reshock (post-reshock) perturbation amplitude, page 63

$a_{b}(t)$

bubble amplitude, page 23

$\bar{A}$

average of $A^{+}$and $A^{-}$, page 27

$a_{b}^{R T(R M)}$

amplitude of a Rayleigh-Taylor (Richtmyer-Meshkov) bubble, page 34

$\alpha$ shock/interface angle, page 44

$A^{-(+)}$

pre-shock (post-shock) Atwood number, page 24

$a_{s}(t)$

spike amplitude, page 23

$a_{s}^{R T(R M)}$

$c_{r}$

amplitude of a Rayleigh-Taylor (Richtmyer-Meshkov) spike, page 34 molar concentration of fluid $r$, defined in Eq. (151), page 52

$c_{s}$

speed of sound, page 120

$\frac{\mathrm{d}}{\mathrm{d} t}$

$\bar{\delta}$

convective derivative, page 16

$\delta^{-(+)}$

average of $\delta^{+}$and $\delta^{-}$, page 27

$\delta(t)$

pre-shock (post-shock) characteristic width of the interface, page 27

$\Delta x$

Dirac delta function, page 24

grid spacing in the $x$-direction, page 47

$(\Delta x)_{\text {coarse }} \quad$ spacing on the coarse grid, page 98

$(\Delta x)_{\text {fine }} \quad$ spacing on the fine grid, page 98

$(\Delta x)_{\text {medium }}$

spacing on the medium grid, page 98

$\Delta y$

grid spacing in the $y$-direction, page 47

$E_{\omega^{\prime \prime} \omega^{\prime \prime}}(t)$

fluctuating enstrophy per unit volume, defined in Eq. (197), page 84

$E_{\omega^{\prime \prime} \omega^{\prime \prime}}(k, t)$

fluctuating enstrophy spectrum, page 78

$E_{\phi \phi}(t)$

statistic, defined in Eq. (193), page 81

$E_{\phi \psi}(k, t)$

energy spectra corresponding to the correlation of $\phi$ and $\psi$, defined in Eq. (186), page 75

$E_{p^{\prime} p^{\prime}}(t)$

density variance, defined in Eq. (199), page 84

$E_{p^{\prime} p^{\prime}}(k, t)$

pressure variance spectrum, page 81 


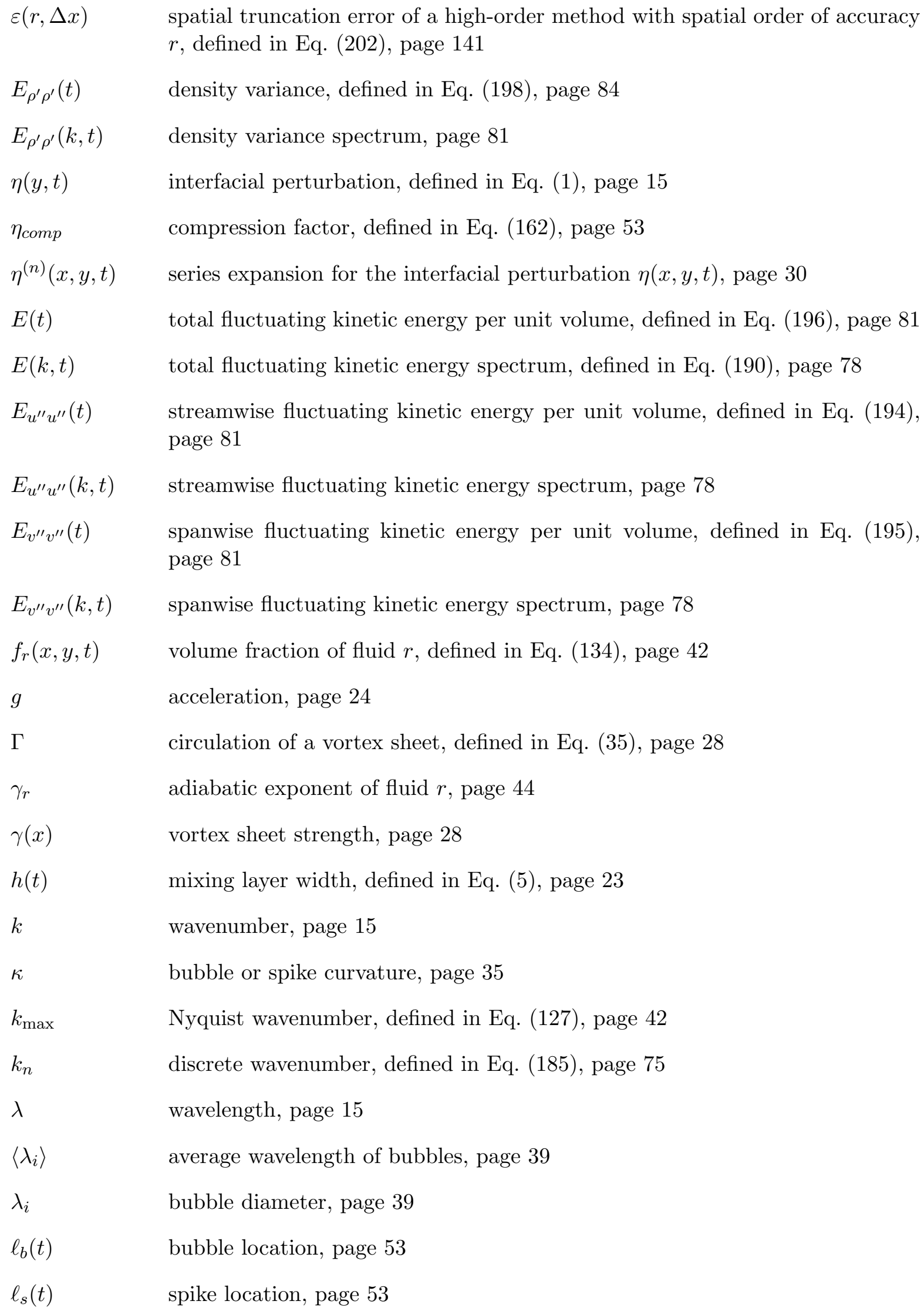




\begin{tabular}{|c|c|}
\hline$M a$ & shock Mach number, page 15 \\
\hline $\bar{M}(x, y, t)$ & mean molecular weight, defined in Eq. (155), page 52 \\
\hline$M_{r}$ & molecular weight of fluid $r$, page 52 \\
\hline$m_{r}(x, y, t)$ & mass fraction offluid $r$, defined in Eq. (151), page 52 \\
\hline$\nabla^{2}$ & Laplacian, page 34 \\
\hline$\omega(x, y, t)$ & vorticity field, page 16 \\
\hline$\omega\left(\lambda_{i}, \lambda_{i+1}\right)$ & bubble merger rate, page 39 \\
\hline$\Omega(t)$ & enstrophy, defined in Eq. (192), page 78 \\
\hline$\Omega(x, y, t)$ & enstrophy density, defined in Eq. (191), page 78 \\
\hline$p(x, y, t)$ & pressure field, page 16 \\
\hline$\Phi(x, y, t)$ & Schlieren function, defined in Eq. (163), page 55 \\
\hline$\langle\phi\rangle(x, t)$ & $\begin{array}{l}\text { average of the function } \phi(x, y, t) \text { over the periodic (spanwise) direction } y \text {, de- } \\
\text { fined in Eq. (170), page } 65\end{array}$ \\
\hline$\widetilde{\phi}(x, t)$ & Favre average of $\phi$, defined in Eq. (172), page 66 \\
\hline$\widehat{\phi}(k, x, t)$ & $\begin{array}{l}\text { Fourier transform of } \phi(x, y, t) \text { in the periodic } y \text {-direction, defined in Eq. (183), } \\
\text { page } 75\end{array}$ \\
\hline$\widehat{\phi}\left(k_{n}, x, t\right)$ & $\begin{array}{l}\text { discrete Fourier transform of } \phi(x, y, t) \text { in the periodic } y \text {-direction, defined in } \\
\text { Eq. (184), page } 75\end{array}$ \\
\hline$\phi(x, y, t)^{\prime}$ & Reynolds fluctuating field, defined in Eq. (171), page 66 \\
\hline$\phi(x, y, t)^{\prime \prime}$ & Favre fluctuating field, defined in Eq. (173), page 66 \\
\hline$\phi_{r}(x, y, t)$ & potential function in fluid $r$, page 30 \\
\hline$\phi_{r}^{(n)}(x, y, t)$ & series expansion for the potential function $\phi_{r}(x, y, t)$, page 30 \\
\hline$\langle\phi\rangle_{x y}(t)$ & volume average of $\phi(x, y, t)$, defined in Eq. (182), page 73 \\
\hline$P_{M}^{N}(z)$ & Padé approximant, defined in Eq. (57), page 31 \\
\hline$\psi^{-(+)}$ & pre-shock (post-shock) growth reduction factor, page 27 \\
\hline$\psi_{1}^{-(+)}$ & pre-reshock (post-reshock) growth reduction factor, page 63 \\
\hline$\psi(x, y)$ & streamfunction, page 29 \\
\hline$P_{m}(t)$ & maximum production fraction, defined in Eq. (179), page 70 \\
\hline$P_{t}(t)$ & production fraction, defined in Eq. (177), page 70 \\
\hline
\end{tabular}


$r$

$R e_{\Delta x}$

$\rho(x, y, t)$

$\bar{\rho}$

$\sigma$

*

$t$

$\tau$

$\theta$

$\theta_{b}$

$\theta_{s}$

$\Theta(t)$

$\theta(x, t)$

$t^{-(+)}$

[u]

$[u]_{1}$

$u(x, y, t)$

$\mathbf{u}(x, y, t)$

$v_{b}\left(\lambda_{i}\right)$

$u_{\text {shock }}$

$v(x, y, t)$

$v_{0}$

$\varpi$

$\varpi_{0}(x / y)$

$\left\langle v_{b}\left(\lambda_{i}\right)\right\rangle$

$v_{b}^{R T(R M)}$

$v_{s}^{R T(R M)}$

$W(t)$ density ratio, page 44

grid Reynolds number, page 120

density field, page 16

average density, page 27

initial perturbation growth, defined in Eq. (46), page 30

complex conjugation, page 75

time

rescaled time, defined in Eq. (161), page 53

power-law growth exponent for the mixing layer width, page 41

power-law growth exponent for the bubble amplitude, page 40

power-law growth exponent for the spike amplitude, page 40

molecular mixing fraction, defined in Eq. (181), page 73

molecular mixing profile, defined in Eq. (176), page 70

time before (after) reshock, page 88

jump in $u$ velocity due to the passage of the shock

jump in $u$ velocity after reshock, page 63

streamwise velocity, page 16

velocity field, page 16

velocity of bubble with diameter $\lambda_{i}$, defined in Eq. (110), page 39

shock velocity, page 26

spanwise velocity, page 16

Richtmyer velocity, defined in Eq. (12), page 25

eigenvalue in the Duff-Harlow-Hirt analysis for diffused interfaces, defined in Eq. (26), page 27

dimensionless merger rate, page 40

mean velocity for bubbles, page 39

velocity of a Rayleigh-Taylor (Richtmyer-Meshkov) bubble, page 36

velocity of a Rayleigh-Taylor (Richtmyer-Meshkov) spike, page 36

integral lengthscale, defined in Eq. (138), page 43 
$w(z) \quad$ complex potential, defined in Eq. (119), page 40

$x$ streamwise coordinate in the direction of shock propagation

$X(x, y, t) \quad$ mole fraction, defined in Eq. (157), page 53

$\Xi(t) \quad$ mixing fraction, defined in Eq. (180), page 73

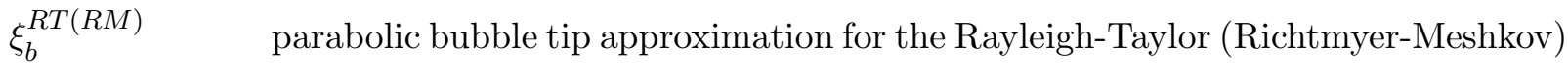
instability, defined in Eq. (87), page 36

$\xi(M a) \quad$ normalized pressure jump, defined in Eq. (148), page 45

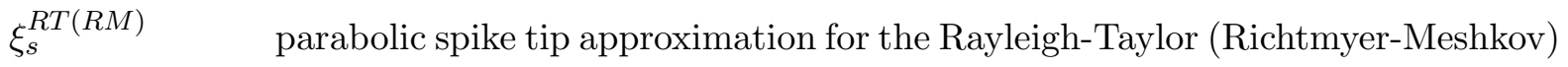
instability, defined in Eq. (87), page 36

$\xi(x, t) \quad$ mixing profile, defined in Eq. (175), page 68

$X_{p}(x, y, t) \quad$ product mole fraction, defined in Eq. (174), page 68

$X_{s} \quad$ parameter for determining $X_{p}(x, y, t)$, page 68

$y \quad$ spanwise coordinate in the periodic direction

$Y(t) \quad$ regularized Heaviside function

$z \quad$ complex variable $z=x+i y$, page 40

$z_{0} \quad$ separation between vortices, page 40 


\section{Introduction}

The Richtmyer-Meshkov instability occurs when perturbations on the interface separating two different fluids grow following the passage of a shock and eventually develop complex structure. The instability derives its name from the linear instability analysis and numerical work of Richtmyer [87], who considered the instability generated by a shock impulsively accelerating a sinusoidally-perturbed interface. The predictions of Richtmyer were subsequently confirmed in shock tube experiments by Meshkov [69]. This instability is of great fundamental interest in fluid dynamics [104, 13], as well as of interest to inertial confinement fusion $[63,77,64,8]$, and to supernovae dynamics $[33,6,7,49,5]$. One of the challenges in understanding the Richtmyer-Meshkov instability is modeling the growth of the mixing layer in the nonlinear phase and following reshock, as well as predicting the statistical properties and dynamics of turbulent mixing induced by the instability.

The classical Richtmyer-Meshkov instability is typically experimentally investigated in shock tubes. Consider a two-dimensional flow, where $x$ is the streamwise direction (the direction of shock propagation) and $y$ is the spanwise direction. Two gases with different densities are initially at rest and separated by a perturbed interface $\eta(y, t=0)$. A multi-mode perturbation can be represented as a superposition of sinusoidal modes

$$
\eta(y, 0)=\sum_{n=1}^{N} a_{n} \sin \left(k_{n} y\right),
$$

where $\left\{a_{n}\right\}$ are the initial amplitudes, and

$$
\begin{aligned}
k_{n} & =\frac{2 \pi}{\lambda_{n}} \\
& =\frac{2 \pi n}{\lambda}
\end{aligned}
$$

are the wavenumbers with wavelengths $\left\{\lambda_{n}\right\}$. Only a single sinusoidal mode

$$
\eta(y, 0)=a_{0} \sin (k y)
$$

is considered in the present investigation (with $k=2 \pi / \lambda$ ), and the gases are taken to be a mixture of air and acetone [denoted air(acetone) in the sequel] and sulfur hexafluoride $\left(\mathrm{SF}_{6}\right)$. A planar shock with Mach number $M a$ is generated in air(acetone) by the rupture of a membrane (or diaphragm), propagates down the shock tube, enters the test section, and interacts with the perturbed interface. In the present work, the evolution of the Richtmyer-Meshkov instability is considered in two spatial dimensions using the HOPE code (see Part 1 of this report [57] for the governing equations and a detailed description of the code and numerical method). A particular validation of the HOPE code using shock refraction theory was also presented in Part 1 [57] of this report. Two-dimensional simulations, analysis and modeling of the Richtmyer-Meshkov instability resulting from the interaction of a planar shock with a perturbed inclined interface, including a study of the effects of shock-interface angle, Mach number, perturbation amplitude, perturbation wavelength, and gas composition are presented in Part 3 of this report [58]. Two- and three-dimensional simulations, analysis, and modeling of the Richtmyer-Meshkov instability are presented in Part 4 of this report [59]. Molecular dissipation and diffusion effects are neglected in the present simulations. Hence, these simulations can be regarded as monotone-integrated (or implicit) large-eddy simulations [12, 30, 31]. 
As the shock passes through the interface, the misalignment of the pressure and density gradients causes a deposition of vorticity through the baroclinic production mechanism illustrated in Fig. 1. Defining the vorticity $\omega=\nabla \times \mathbf{u}$, where $\mathbf{u}$ is the velocity, the vorticity evolution equation (shown here for three dimensions and in the absence of dissipation terms)

$$
\frac{\mathrm{d} \omega}{\mathrm{d} t}=(\omega \cdot \nabla) \mathbf{u}-\omega \nabla \cdot \mathbf{u}+\frac{\nabla \rho \times \nabla p}{\rho^{2}}
$$

describes the dynamics of vorticity generation, where $\mathrm{d} / \mathrm{d} t=\partial / \partial t+\mathbf{u} \cdot \nabla$ is the convective derivative, $\rho$ is the density, and $p$ is the pressure. The first term on the right side is the vortexstretching term, which is zero in the present two-dimensional investigation, as the vorticity and velocity field are orthogonal. Vortex stretching is a fundamental mechanism in the dynamics of turbulence and distinguishes 'two-dimensional turbulence' [53] from actual three-dimensional turbulence. In particular, vortex stretching is associated with the cascade of energy from large scales to smaller scales through an inertial subrange at sufficiently large Reynolds numbers. In two-dimensional flows, the energy transfer is predominantly from small scales to larger scales, resulting in the generation of larger and more coherent structures than observed in three-dimensional flows [22]. Vortex stretching enhances dissipation, resulting in more diffuse and smaller scale structures in three dimensions. Thus, the properties of turbulent mixing are expected to be significantly different in two and three dimensions. These differences will be examined in detail in Part 4 [59] of this report. The second term on the right side is the compression term, and does not contribute significantly to the vorticity evolution. The third term on the right side is the baroclinic production term, and constitutes the principal mechanism of vorticity generation by the Richtmyer-Meshkov instability. This term is large when the shock passes through the interface and when waves interact with the interface. Following the passage of the shock, the perturbed interface is set in motion along the direction of shock propagation, a reflected shock returns back into the air(acetone) gas, and a transmitted shock enters the $\mathrm{SF}_{6}$ gas.

The vorticity baroclinically deposited on the interface by the shock drives the evolution of the instability, with spikes of the heavier fluid $\left(\mathrm{SF}_{6}\right)$ penetrating the lighter fluid [air(acetone)] and bubbles of the lighter fluid "rising" in the heavier fluid. When the fluids are miscible, molecular mixing occurs between these interpenetrating spikes and bubbles, generating a mixing layer and a topologically-complex flow. In the present investigation, explicit molecular mixing is not modeled; instead, numerical diffusion across the interface models the 'molecular mixing' process. At late times, the vorticity coalesces into strong cores causing the characteristic mushroom roll-ups to form, as illustrated in Fig. 1. Additional deposition of vorticity and enhanced mixing occurs when the interface interacts with another shock wave, as in the configuration considered here. The transmitted shock from the initial shock-interface interaction reflects from the end wall of the shock tube test section and interacts with the evolving layer, as illustrated in Fig. 2. This second interaction (referred to as reshock in the sequel) deposits vorticity of opposite sign so that bubbles transform into spikes and vice versa in a process called inversion. The inversion process induces the formation of additional complex structures, with additional disorganized small-scale flow features observed at late times, as shown in Fig. 3. Following reshock, the interface changes its direction of motion and now moves away from the end wall of the test section: a transmitted shock enters the air(acetone) and a reflected rarefaction returns into the $\mathrm{SF}_{6}$. Unlike in the case of the first shock-interface interaction, the reflected wave is now a rarefaction wave, as the shock refracts from the heavier $\mathrm{SF}_{6}$ gas into the lighter air(acetone) gas. The rarefaction wave is reflected from the end wall of the test section 
and interacts with the evolving interface, resulting in the formation of additional complex, small-scale structures. Throughout the evolution of the instability, both prior to and following reshock, shear due to the secondary Kelvin-Helmholtz instability [28, 29, 67] develops at the interface. This secondary instability further enhances the development of a complex topology with increasing surface area, eventually resulting in a mixing layer that becomes turbulent at sufficiently large Reynolds numbers. The single-mode Richtmyer-Meshkov instability with reshock is considered in the present work, as it offers the possibility to observe two phases of the instability development and their coupling: the nonlinear phase prior to reshock, and the post-reshock phase. As summarized below in $\S 1.1$ and 1.2, this flow configuration has not been extensively studied either experimentally or numerically.

\subsection{Previous single-mode Richtmyer-Meshkov instability experiments with reshock}

Many experiments have been performed to investigate the properties of the Richtmyer-Meshkov instability (see Brouillette [13] for a recent review). However, most of the experiments conducted to date only consider the evolution of the instability in the linear and nonlinear regimes. Relatively few experiments have been performed to study the dynamics of the flow following reshock. Briefly summarized below are single-mode, compressible experiments with reshock in a planar geometry, which are relevant to the present investigation (multi-mode experiments are summarized in Part 4 [59] of this report). The present discussion is limited to classical fluid (shock tube) experiments and does not consider high-energy density (laser) experiments.

Houas and Chemouni [43] performed shock tube experiments using $\mathrm{CO}_{2} / \mathrm{He}, \mathrm{CO}_{2} / \mathrm{Ar}$, and $\mathrm{CO}_{2} / \mathrm{Kr}$ over a range of shock Mach numbers $M a=2-4.7$ to determine the power-law governing the width of the mixing layer before and after reshock. The measurements were compared against the linear [71] and $t^{2 / 3}[10]$ growth power-laws, and it was concluded that the data was in good agreement with the $t^{2 / 3}$ law both before and after reshock. Sadot et al. [90] performed a shock tube experiment using air $/ \mathrm{SF}_{6}$ with $\lambda=2.6 \mathrm{~cm}, a_{0}=0.2 \mathrm{~cm}$, and $M a=1.3$. Collins and Jacobs [23] performed shock tube experiments using air(acetone) $/ \mathrm{SF}_{6}$ with $\lambda=5.93 \mathrm{~cm}, a_{0}=0.229 \mathrm{~cm}$ and $0.183 \mathrm{~cm}$ for $M a=1.11$ and 1.21 , respectively. The experimental amplitude growth prior to reshock was compared to the predictions of models. These experiments are described in more detail in $\S 3.1$. The quantitative data obtained from these experiments was mainly limited to perturbation amplitude growth.

\subsection{Previous numerical simulations of single-mode Richtmyer-Meshkov in- stability with reshock}

The vast majority of numerical simulations of single- and multi-mode Richtmyer-Meshkov instabilities to date have considered the flow evolution initiated by only a single shock-interface interaction. As in the case of experiments, very few simulations have considered the effects of reshock on an evolving interface. Briefly summarized below are single-mode, compressible simulations with reshock in a planar geometry, which are relevant to the present investigation (multi-mode simulations are summarized in Part 4 [59] of this report). The present discussion is limited to classical fluid (shock tube) simulations and does not consider simulations in converging geometry.

Mikaelian [73] performed two-dimensional arbitrary Lagrangian-Eulerian simulations of gas configurations consisting of three layers, $1 / 2 / 1$, with fluid 1 representing semi-infinite layers of 


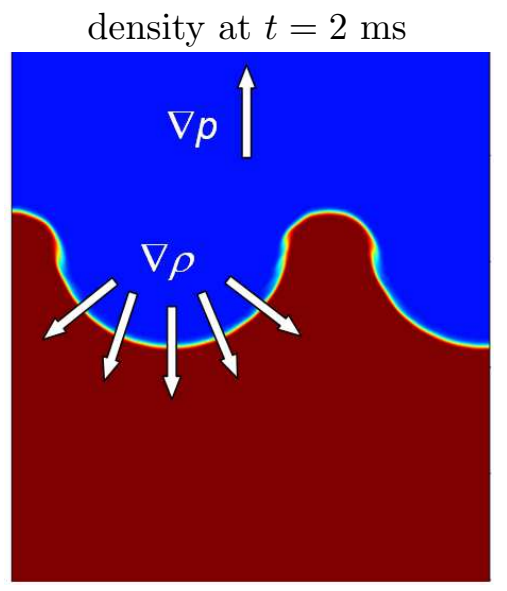

density at $t=5 \mathrm{~ms}$

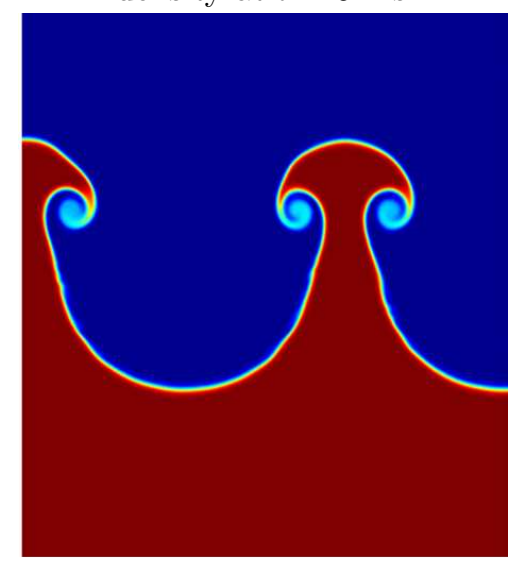

vorticity at $t=2 \mathrm{~ms}$

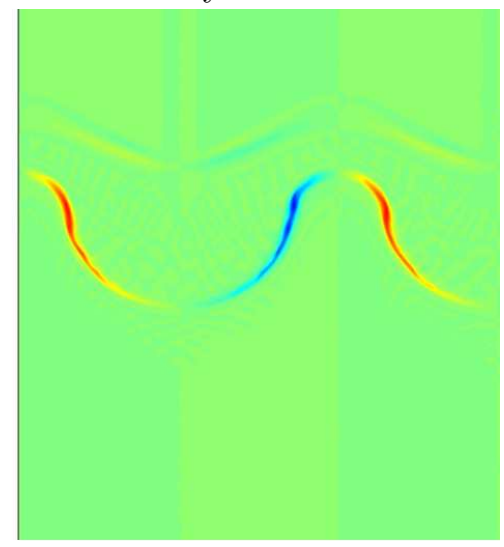

vorticity at $t=5 \mathrm{~ms}$

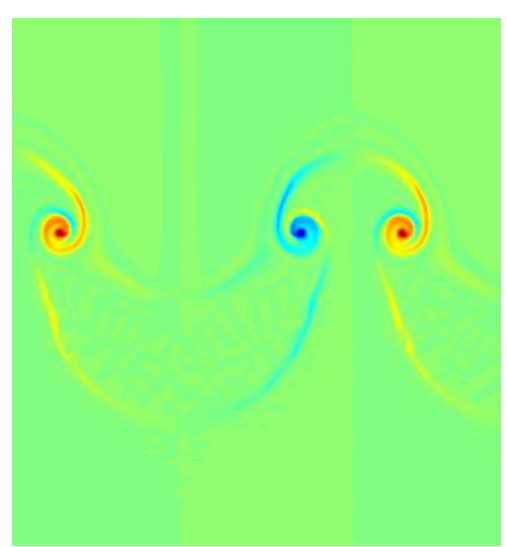

Figure 1: The Richtmyer-Meshkov instability occurs when perturbations on an interface separating two fluids with different properties grow following the passage of a shock. The vorticity deposited baroclinically through the misalignment of the density and pressure gradients drives the evolution of the instability. As the instability develops, spikes of heavier fluid penetrate into the lighter fluid and bubbles of the lighter fluid penetrate into the heavier fluid. The vorticity coalesces into vortices with strong cores forming the characteristic "mushrooms" at late times. The images are taken from the simulation described in $\S 3$. 

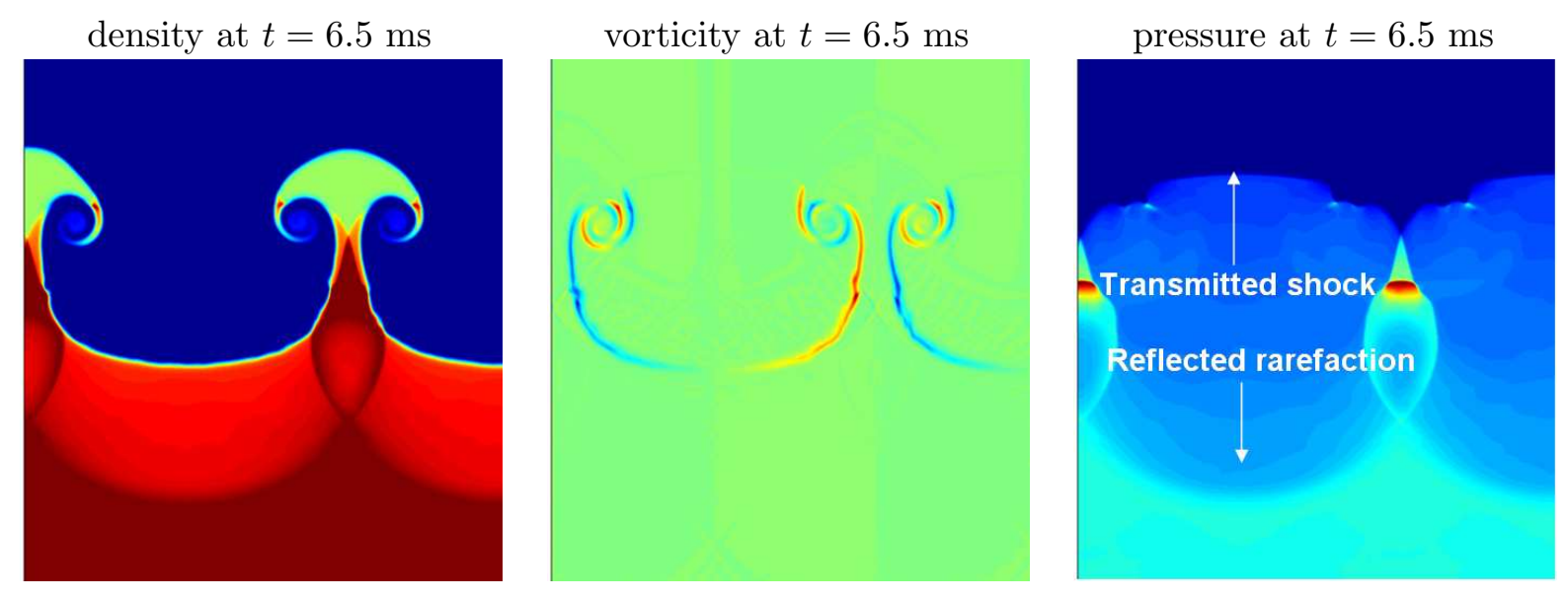

Figure 2: The single-mode Richtmyer-Meshkov instability during reshock. The reflected shock compresses the interface and deposits vorticity of opposite sign on the interface. Note the reflected rarefaction wave and the transmitted shock wave in the pressure. The images are taken from the simulation described in $\S 3$.
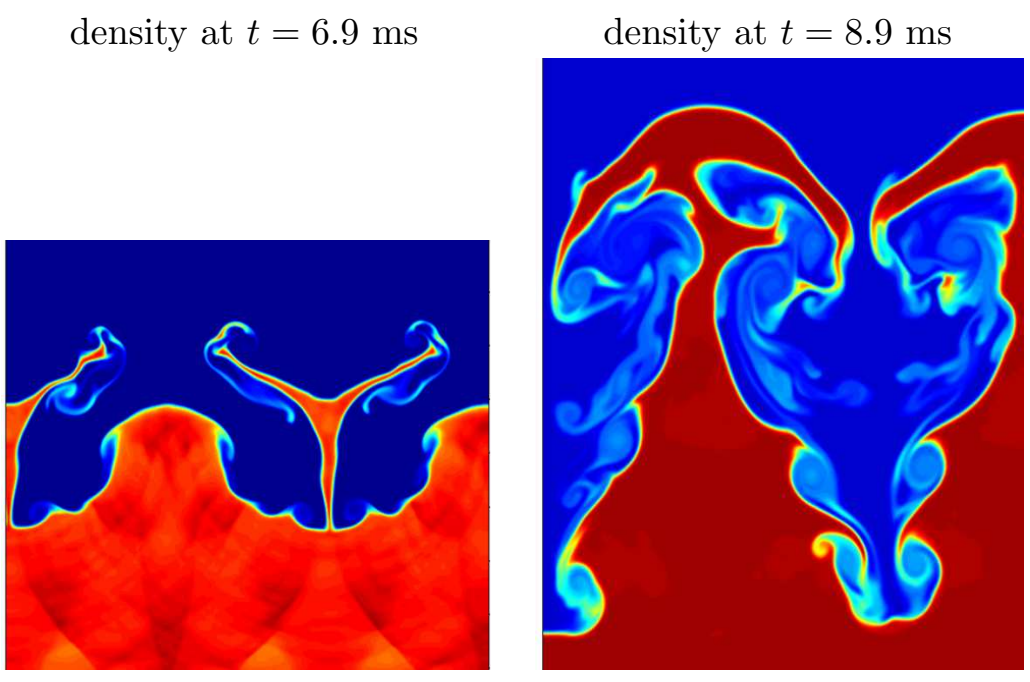

Figure 3: Following reshock of the evolving interface, bubbles transform into spikes and vice versa during the inversion process, causing additional structures to form. At late times, the roll-ups develop more disorganized, small-scale structures. The images are taken from the simulation described in $\S 3$. 
air and fluid 2 representing a finite-thickness layer of freon, $\mathrm{SF}_{6}$, or He having perturbations either on the upstream or downstream side. These perturbations were in phase (sinuous) or out of phase (varicose). The shock Mach number was 1.5. The primary purpose of the simulations was to investigate freeze-out, interface coupling, and feedthrough. Sadot et al. [90] compared the amplitude growth from their single-mode reshock experiment to numerical simulation data and found very good agreement prior to the arrival of the rarefaction wave from the end wall. As in the case of experiments, the quantitative data obtained from these simulations was mainly limited to the consideration of perturbation amplitude growth.

\subsection{Objectives of the current investigation}

A central objective of the present work is to establish a systematic procedure to investigate the dynamics of the mixing process induced by the Richtmyer-Meshkov instability, and more generally by complex hydrodynamic flows. The methods used are adapted from classical investigations of turbulence and turbulent mixing, and synthesize high-resolution numerical simulation data, theoretical models for instability growth, and available experimental data. This procedure results in:

1. the application of a modern, high-resolution, flexible numerical method that has been validated against available experimental data;

2. a numerical database that provides quantities that can be compared to model predictions and to experimental measurements, as well as quantities that have not been modeled (or are difficult to model) or are not available experimentally;

3. numerical data for configurations extended to times beyond what is possible to achieve experimentally, or for configurations that are difficult to achieve experimentally;

4. a systematic understanding of the important effects of spatial resolution and formal order of the method on quantities of interest to modeling the instability evolution and mixing.

This study is part of a larger, longer-term program aimed at:

1. developing improved theoretical models for instability growth in the nonlinear regime, as well as for the evolution following reshock;

2. investigating closure models for ensemble-averaged descriptions of turbulent transport and mixing, as well as for the development of subgrid-scale models for large-eddy simulations;

3. improving the numerical methods used to simulate complex hydrodynamic flows induced by shocks;

4. aiding the design of new experimental configurations and new experimental diagnostics.

This report is organized according to three principal components. First, a comprehensive review of the main linear and nonlinear models for single- and multi-mode perturbation amplitude growth is presented in $\S 2$, including impulsive, vortex, perturbation, potential flow, and asymptotic power-law growth models. Models for diffuse and reshocked interfaces are also reviewed. Two-dimensional numerical simulations of the single-mode Richtmyer-Meshkov 
instability with reshock using the fifth-order WENO method (using the HOPE code) and a uniform grid resolution based on 256 points per initial perturbation wavelength are performed. The initial conditions and computational domain for the simulations are adapted from the Mach 1.21 air(acetone)/ $\mathrm{SF}_{6}$ - shock tube experiment of Collins and Jacobs [23]. The interface evolution (density) is compared to the experimental PLIF images of Collins and Jacobs before and after reshock in $\S 3$. In addition, the mixing layer width from the simulation before and after reshock is compared to the experimental data and to the predictions of the analytical, semi-analytical, and phenomenological models summarized in $\S 2$.

Second, a comprehensive quantitative analysis of the local and global properties of mixing is presented in $\S 4$. The analysis characterizes the mixing process along the direction of shock propagation using mole fractions and a fast kinetic reaction model. The modal distribution of energy in the mixing layer is quantified using a Fourier (spectral) analysis of the fluctuating kinetic energy (and its streamwise and spanwise components) and enstrophy, as well as the pressure and the density variance. The evolution of mixing is characterized using mixing fractions before and after reshock up to time $t=18 \mathrm{~ms}$. Finally, statistics (wavenumberintegrated energy spectra) are considered to understand the time-evolution of energy present in the fluctuations. Furthermore, to investigate the decay of fluctuations in the mixing layer in the absence of additional waves interacting with the evolving interface following reshock, the boundary condition at the end of the computational domain (corresponding to the end wall of the test section) is varied from reflecting to outflow to allow the reflected rarefaction wave to exit the domain. At late time, this case is referred to as the decay regime, as distinguished from the quasi-decay regime occurring when reflected waves interact with the evolving interface following reshock. Comparisons of mole fractions, spatially-integrated mixing profiles and fractions, and statistics between the reflecting and outflow boundary condition cases are presented and discussed.

Third, a comprehensive investigation of the dependence of the mixing layer width and of mixing quantities on the order of reconstruction and grid resolution is presented in $\S 5$. The results from two-dimensional numerical simulations using the third-, fifth-, and ninth-order WENO reconstruction and three different uniform grid resolutions corresponding to 512, 256, and 128 points per initial perturbation wavelength are compared. Simulations with different grid resolutions and orders of reconstruction have different numerical diffusion properties introduced by the algorithm. In particular, high-order, high-resolution simulations have lower numerical diffusion than low-order, low-resolution simulations. The differences in the timeevolution and structure of the mole fractions, spectra, mixing fractions, and statistics are investigated before and after reshock. A temporal progression of the density and vorticity fields is presented at intervals of $1 \mathrm{~ms}$ for the three orders of reconstruction and three grid resolutions in $\S 5.7$. 



\section{Models for the perturbation amplitude and mixing layer growth and for circulation deposition}

The prediction and modeling of the mixing layer growth in the nonlinear and turbulent regimes resulting from the Richtmyer-Meshkov instability is of great interest. An overview of the principal models categorized according to the underlying physical assumptions on the flow is presented in this section. Note that it is implicitly assumed in all of these models that molecular dissipation and diffusion effects, as well as surface tension and other effects, are negligible. These models all have important limitations and a limited domain of applicability, but represent an effort to better understand the fundamental aspects of Richtmyer-Meshkov instability growth into the nonlinear regime.

Figure 4 shows an illustration of the bubble and spike amplitudes $a_{b}$ and $a_{s}$, respectively, and the mixing layer width $h$. The blue contour shows a typical early-time evolution of the interface induced by the Richtmyer-Meshkov instability. The spikes penetrate into the lighter fluid and roll up, while bubbles "rise" into the heavier fluid. The solid red line shows the location of the shocked, unperturbed interface used as a reference for the measurements of the bubble and spike amplitudes. The distance from the unperturbed interface to the tip of the bubble represents the bubble amplitude $a_{b}$, while the distance from the unperturbed interface to the tip of the spike represents the spike amplitude $a_{s}$. The mixing layer width is defined as the sum of the bubble and spike amplitudes

$$
h(t)=a_{b}(t)+a_{s}(t)
$$

The mixing layer amplitude predicted by the models presented in this section is the average of the bubble and spike amplitude

$$
\begin{aligned}
a(t) & =\frac{a_{b}(t)+a_{s}(t)}{2} \\
& =\frac{h(t)}{2},
\end{aligned}
$$

i.e., one-half the mixing layer width.

Impulsive models based on representing the shock as a $\delta$-function acceleration are reviewed in $\S 2.1$. Models based on representing the vorticity deposited by the shock as point vortices are reviewed in $\S 2.2$. Models based on asymptotic expansions of the perturbed compressible fluid equations are reviewed in $\S 2.3$. Models based on potential theory are reviewed in $\S 2.4$. Scaling laws for multi-mode initial conditions are reviewed in $\S 2.5$. Finally models for the deposition of circulation by a shock are reviewed in $\S 2.6$.

\subsection{Impulsive models}

Impulsive models based on representing the shock as an instantaneous $\delta$-function acceleration are briefly reviewed and summarized here. Impulsive models for the Richtmyer-Meshkov instability were developed by adapting existing models for the Rayleigh-Taylor instability. These models predict a linear growth in time for the mixing layer that captures the early stages of the instability evolution before nonlinear effects become important. 


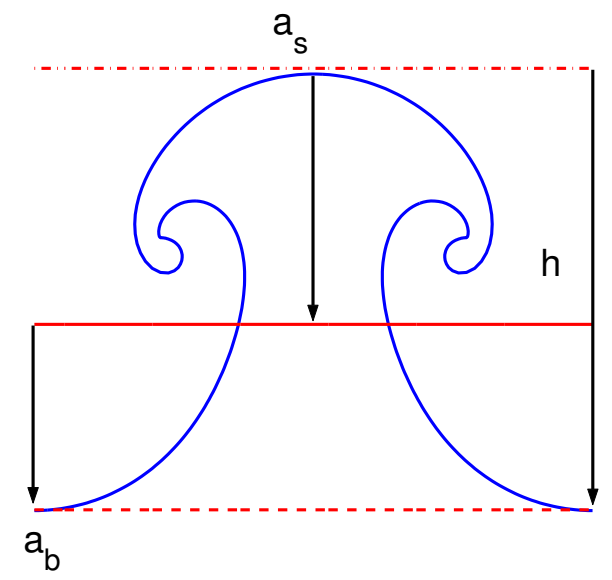

Figure 4: The solid blue line represents a typical interface evolving according to the RichtmyerMeshkov instability with spikes penetrating the lighter fluid and rolling up, and bubbles rising in the lighter fluid. The solid red line is the location of the shocked unperturbed interface and is used as the reference to measure the bubble and spike amplitudes $a_{b}$ and $a_{s}$. The mixing layer width is $h=a_{s}+a_{b}$ and the amplitude of the perturbation is $a=\left(a_{s}+a_{b}\right) / 2$. The image is from a simulation of the Richtmyer-Meshkov instability using a vortex method [56].

\subsubsection{The Richtmyer model}

The first impulsive model proposed to predict the growth of the single-mode perturbation amplitude is due to Richtmyer [87]. Richtmyer modified earlier work by Taylor [96] for the growth of a small perturbation with amplitude $a(t)$ and wavenumber $k$ when a dense fluid is accelerated continuously into a lighter fluid, and the fluids are initially separated by a singlemode interfacial perturbation (the single-mode Rayleigh-Taylor instability). Replacing the constant gravitational acceleration $g$ in the Taylor result

$$
\frac{\mathrm{d}^{2} a}{\mathrm{~d} t^{2}}=g A k a
$$

where $A=\left(\rho_{2}-\rho_{1}\right) /\left(\rho_{2}+\rho_{1}\right)$ is the Atwood number and $\rho_{1}$ and $\rho_{2}$ are the densities of the lighter and heavier fluid, respectively] by the impulsive acceleration that the interface undergoes after the passage of the shock

$$
g=[u] \delta(t)
$$

where $[u]$ is the jump in the interface velocity due to shock passage, and integrating Eq. (7) gives

$$
\frac{\mathrm{d} a}{\mathrm{~d} t}=k[u] A^{-} a_{0},
$$

where $a_{0}^{-} \equiv a(0)$ is the initial (pre-shock) perturbation amplitude and $A^{-}$is the pre-shock Atwood number. As the right side of this equation is constant, the Richtmyer impulsive model predicts linear growth according to

$$
a(t)=\left(1+k[u] A^{-} t\right) a_{0}^{-} .
$$


Richtmyer found good agreement between the prediction of this model and the amplitude growth obtained by the direct numerical solution of the full set of perturbation equations [87] in the case when the shock propagates from a lighter fluid into a heavier fluid $\left(A^{-}>0\right)$. Even better agreement was obtained when the pre-shock Atwood number, $A^{-}$, was replaced by the post-shock Atwood number, $A^{+}$, and the pre-shock amplitude, $a_{0}^{-}$, was replaced by the post-shock amplitude, $a_{0}^{+}$:

$$
\frac{\mathrm{d} a}{\mathrm{~d} t}=v_{0}
$$

where the Richtmyer velocity is

$$
v_{0} \equiv k[u] A^{+} a_{0}^{+},
$$

so that

$$
\begin{aligned}
a(t) & =a_{0}^{+}+v_{0} t \\
& =\left(1+k[u] A^{+} t\right) a_{0}^{+} .
\end{aligned}
$$

\subsubsection{The Meyer-Blewett model}

Meyer and Blewett [70] found that their experimental amplitude growth data for shocks propagating from heavy to light gases was better modeled if the post-shock initial amplitude $a_{0}^{+}$ was replaced by the pre-shock initial amplitude, $a_{0}^{-}$. For this reason, Meyer and Blewett empirically replaced the initial post-shock amplitude in the Richtmyer formulation Eq. (11) by the average of the pre- and post-shock amplitudes

$$
\frac{\mathrm{d} a}{\mathrm{~d} t}=k[u] A^{+} \frac{a_{0}^{+}+a_{0}^{-}}{2}
$$

so that

$$
a(t)=\left(1+k[u] A^{+} t\right) \frac{a_{0}^{+}+a_{0}^{-}}{2} .
$$

\subsubsection{The Fraley perturbation solution}

Fraley [34] presented an analytic solution to the linearized perturbation equations in the case of a reflected shock wave. The linearized perturbation equations were considered first by Richtmyer [87], who solved them numerically. Fraley reconsidered the perturbation equations for a single-mode initial perturbation and solved the equations by taking the Laplace transform in time. For weak shocks the solution is given by (see [13])

$$
\begin{gathered}
\frac{\mathrm{d} a}{\mathrm{~d} t}=k[u] a_{0}^{-}\left[A^{-}+\epsilon \frac{F\left(c, A^{-}\right)}{\gamma_{1}}\right] \\
F(c, A)=\left\{\frac{(c-1)^{2}}{2}-\frac{1+A}{1-A}-c+\frac{1}{c}\left[\frac{(1+A)^{2}}{1-A}+(1-A) c^{2}\right]\right\} \frac{1-A}{c+1} \\
c=\sqrt{\frac{\left(1+A^{-}\right) \gamma_{2}}{\left(1-A^{-}\right) \gamma_{1}}} .
\end{gathered}
$$


with the Richtmyer model recovered with $\epsilon \rightarrow 0$. Thus,

$$
a(t)=\left\{1+k[u]\left[A^{-}+\epsilon \frac{F\left(c, A^{-}\right)}{\gamma_{1}}\right] t\right\} a_{0}^{-} .
$$

This solution was first recognized by Mikaelian [72] as the most accurate solution for the initial growth of the perturbation. In particular, Mikaelian showed that when $A^{+}$is chosen to be zero, the Richtmyer formula (11) predicts a zero growth rate - the so-called "freeze-out". However, numerical experiments do not show a freeze-out for $A^{+}=0$. Instead, freeze-out is observed when the values are chosen so that $\mathrm{d} a / \mathrm{d} t=0$.

\subsubsection{The Vandenboomgaerde et al. model}

Vandenboomgaerde, Mügler and Gauthier [99] developed an amplitude growth model based on modifying the impulsive acceleration assumption of Richtmyer [87]. Returning to the RayleighTaylor instability result for incompressible flows, Eq. (7), the constant terms for the acceleration $g$, Atwood number $A$, and amplitude $a$ were replaced by linearly time-varying values from the pre- to post-shock quantities. The linear variation occurs as the shock crosses the interface between times $t_{0}^{-}=-a_{0}^{-} /\left(2 u_{\text {shock }}\right)$ and $t_{0}^{+}=a_{0}^{-} /\left(2 u_{\text {shock }}\right)$. Therefore,

$$
\begin{gathered}
g(t)=\frac{u_{\text {shock }}[u]}{a_{0}^{-}} Y_{-}\left(1-Y_{+}\right), \\
A(t)=\left(1-Y_{-}\right) A^{-}+Y_{-}\left(1-Y_{+}\right)\left[\frac{u_{\text {shock }}\left(A^{+}-A^{-}\right) t}{a_{0}^{-}}+\frac{A^{+}+A^{-}}{2}\right]+Y_{+} A^{+}, \\
a(t)=\left(1-Y_{-}\right) a_{0}^{-}+Y_{-}\left(1-Y_{+}\right)\left[\frac{u_{\text {shock }}\left(a_{0}^{+}-a_{0}^{-}\right) t}{a_{0}^{-}}+\frac{a_{0}^{+}+a_{0}^{-}}{2}\right]+Y_{+} a_{0}^{+},
\end{gathered}
$$

where $Y_{ \pm}=Y\left(t-t_{0}^{ \pm}\right)$, and $Y(t)$ is the regularized Heaviside function

$$
Y(t) \equiv\left\{\begin{array}{lc}
0 & t<-\delta \\
\frac{t+\delta}{2 \delta}+\sin \left(\frac{\pi t}{\delta}\right) & -\delta \leq t \leq \delta \\
1 & t>\delta
\end{array}\right.
$$

Substituting Eqs. (19)-(21) into Eq. (7) and integrating gives

$$
\frac{\mathrm{d} a}{\mathrm{~d} t}=\frac{1}{2} k[u]\left(A^{+} a_{0}^{+}+A^{-} a_{0}^{-}\right)-\frac{1}{6} k[u]\left(A^{+}-A^{-}\right)\left(a_{0}^{+}-a_{0}^{-}\right),
$$

so that

$$
a(t)=a_{0}^{-}+k[u]\left\{\frac{A^{+} a_{0}^{+}+A^{-} a_{0}^{-}}{2}-\frac{\left(A^{+}-A^{-}\right)\left(a_{0}^{+}-a_{0}^{-}\right)}{6}\right\} t .
$$

Note that the second term on the right side is generally very small, and is therefore typically neglected. The Meyer-Blewett formula (15) takes into account the variation in amplitude for a shocked interface, whereas the above formulation also considers the change in Atwood number.

To determine the range of validity of Eq. (23), the predicted amplitude is compared to the exact solution of the linearized perturbation equations provided by Fraley [34] using a normalized growth rate analysis. In this analysis [72], the normalized growth rate obtained via Eq. (17) and Eq. (23) are plotted as a function of the shock strength. The region over which the two formulae agree constitutes the region of validity for the Vandenboomgaerde model. Such an analysis can also be applied to other amplitude growth models. 


\subsubsection{The Brouillette-Sturtevant model for diffuse interfaces}

Brouillette and Sturtevant [15] modified the Richtmyer model to account for the presence of a diffuse, perturbed interface separating two gases. Their model is based on the classical analysis of Duff, Harlow and Hirt [32] concerning the evolution of the Rayleigh-Taylor instability in fluids separated by a thick, diffuse interface.

The Duff, Harlow, and Hirt analysis begins with the linear eigenvalue equation for the perturbation velocity $u$ corresponding to a sinusoidally-perturbed, arbitrary density profile subject to a gravitational acceleration (see [20])

$$
\begin{aligned}
\frac{\mathrm{d}}{\mathrm{d} x}\left(\rho \frac{\mathrm{d} u}{\mathrm{~d} x}\right) & =u k^{2}\left(\rho-\frac{g}{\varpi^{2}} \frac{\mathrm{d} \rho}{\mathrm{d} x}\right) \\
\frac{\mathrm{d}^{2} a}{\mathrm{~d} t^{2}} & =\varpi^{2} a
\end{aligned}
$$

where the eigenvalue $\varpi^{2}=g A k$ is appropriate for a discontinuous interface. For a diffuse interface, Duff, Harlow, and Hirt heuristically proposed the eigenvalue

$$
\varpi^{2}=\frac{g A k}{\psi},
$$

where $\psi$ is the growth reduction factor and is, in general, a function of the interface width and Atwood number. Substituting this expression into Eq. (25), $\psi$ becomes the new eigenvalue to be determined. Specifically, the equation for the amplitude evolution becomes

$$
\frac{\mathrm{d}^{2} a}{\mathrm{~d} t^{2}}=\frac{g A k}{\psi} a
$$

Substituting the gravitational acceleration by an impulsive acceleration of Eq. (8) allows the resulting equation to be integrated directly to account for the width of the interface in the Richtmyer model:

$$
\frac{\mathrm{d} a}{\mathrm{~d} t}=\frac{k[u] A^{+}}{\psi} a_{0}^{+} .
$$

The value of $\psi$ is obtained by solving the eigenvalue problem with the diffuse density profile

$$
\rho(x)=\bar{\rho}\left[1+\bar{A} \operatorname{erf}\left(\frac{x}{\bar{\delta}}\right)\right]
$$

where $\bar{\rho}=\left(\rho_{1}+\rho_{2}\right) / 2, \bar{A}=\left(A^{-}+A^{+}\right) / 2$, and $\bar{\delta}=\left(\delta^{-}+\delta^{+}\right) / 2$. Note that $\delta$ is the characteristic width of the interface, and is taken as the maximum slope of the density profile at a time $\tau$ after molecular diffusion begins. The growth reduction factor is larger for smaller Atwood number, and $\psi=1+\pi \bar{\delta} / \lambda$ in the limit $\bar{A} \rightarrow 0$. In the limit of a thin interface with $\bar{\delta} / \lambda=0$, it follows that $\psi=1$. Also, $\psi=1$ in the limit $\bar{A} \rightarrow 1$.

When the width of the interface is larger than the wavelength of the perturbation, $\bar{\delta} / \lambda>1$,

$$
\psi=1+C \frac{\bar{\delta}}{\lambda}
$$

where $C$ is a constant. This yields

$$
\frac{\mathrm{d} a}{\mathrm{~d} t}=\frac{2 \pi}{\bar{\delta}} \frac{A^{+}[u] a_{0}^{+}}{C}
$$


so that

$$
a(t)=\left(1+\frac{2 \pi}{\bar{\delta}} \frac{A^{+}[u] a_{0}^{+}}{C} t\right) a_{0}^{+} .
$$

This model is easily integrated into other models by the substitution

$$
k \longrightarrow \frac{k}{\psi} \text {. }
$$

Note that Collins and Jacobs [23] also included the growth reduction factor $\psi$ in their comparison of model predictions to their experimental data. The value $\psi=1.08$ is used in the comparisons of model predictions to numerical simulation data later in this report, and is taken from Collins and Jacobs [23].

\subsection{The Jacobs-Sheeley vortex model}

Jacobs and Sheeley [47] developed a vortex model for the amplitude growth measured in their incompressible Richtmyer-Meshkov instability experiments. This model was presented together with other models to reconcile their experimental results with the model predictions available at the time. Jacobs and Sheeley considered the 'impulsive Richtmyer-Meshkov instability' observed when a tank containing two liquids is impulsively accelerated by rapidly decelerating it after a short drop. The use of liquids allows sharper initial interfaces without the presence of a membrane, as liquids do not diffuse very rapidly. Furthermore, the experimental configuration with the free-fall component removes any effect from gravity.

Jacobs and Sheeley argued that the appearance of mushrooms in the Richtmyer-Meshkov instability is a manifestation of the coalescence of the initial sheet of vorticity into vortices with well-defined cores located at midpoints along the interface. The strength of the initial vortex sheet $\gamma$ can be computed based on linear analysis as

$$
\gamma(x)=-2 v_{0} \sin (k x),
$$

where $v_{0}$ is the initial growth rate of the interface and $k$ is the perturbation wavenumber. As the vortex sheet coalesces into single point vortices, it is possible to determine the circulation $\Gamma$ as

$$
\begin{aligned}
\Gamma & =\int_{0}^{\pi / k} \gamma(x) \mathrm{d} x \\
& =-\frac{4}{k} v_{0} .
\end{aligned}
$$

Additional mechanisms for the production of vorticity can be neglected, as they are typically much smaller compared to the baroclinic production mechanism arising from the passage of the shock.

Assume that a periodic array of point vortices with alternating signs is initially located along the interface at points midway between the peaks and troughs of the initial perturbation. The velocity field generated by this set of vortices is determined by the streamfunction

$$
\psi(x, y)=\frac{\Gamma}{4 \pi} \ln \left[\frac{\cosh (k y)+\sin (k x)}{\cosh (k y)-\sin (k x)}\right] .
$$

As the instability evolves in time, the flow will distort the interface and wrap it around the vortex cores. The growth of the mixing layer is determined by the velocity of the point located 
between two successive vortices. The component of the velocity normal to the array of vortices is

$$
\begin{gathered}
v(x, y)=-\frac{\partial \psi}{\partial x} \\
=-\frac{k \Gamma}{2 \pi} \frac{\cosh (k y) \cos (k x)}{\cosh ^{2}(k y)-\sin ^{2}(k x)} .
\end{gathered}
$$

At the midpoints $x= \pm n \pi / k$, the normal velocity is given by

$$
v(y)= \pm \frac{1}{2 \pi} \frac{k \Gamma}{\cosh (k y)} .
$$

At the midpoints, the vertical position $y(t)$ is also equivalent to the amplitude of the mixing layer $a(t)$. Therefore, the initial value problem for the amplitude

$$
\frac{\mathrm{d} a}{\mathrm{~d} t}=v(t), a(0)=a_{0}^{+}
$$

is obtained, which can be solved via a substitution from Eq. (35): the result is

$$
a(t)=\frac{1}{k} \sinh ^{-1}\left[\frac{2}{\pi} k v_{0}^{+} t+\sinh \left(k a_{0}^{+}\right)\right] .
$$

It was found that this model underestimates the observed data, but gives a late-time logarithmic scaling of the amplitude, corresponding to a $1 / t$ scaling of the velocity. To address the discrepancy between the model prediction and the data, it was suggested that this model becomes valid when the initial sheet of vorticity has had sufficient time to coalesce into a single vortex. At this point, the interface has already developed for some time and, therefore, a larger value for $k a_{0}^{+}$must be used. With this correction, the model overestimates the data, but somewhat better agreement is obtained at late times.

\subsection{Perturbation models}

Models based on the asymptotic expansion of the perturbation equations are reviewed here. These models generate asymptotic series with limited radii of convergence: the convergence can be improved using Padé approximants.

\subsubsection{The Zhang-Sohn model}

Zhang and Sohn [107] developed a model to investigate the growth rate of a Richtmyer-Meshkov unstable interface, valid for compressible fluids from early to late times in the case of a reflected shock (light-to-heavy transition). The dynamics of the initially-perturbed interface are modeled using the linear, compressible Euler equations for early times and using the nonlinear, incompressible equations for later times.

Let $y=\eta(x, t)$ denote the initial perturbation, and let $\phi_{1}(x, y, t)$ and $\phi_{2}(x, y, t)$ denote the velocity potentials for the flows in the inviscid, irrotational fluids 1 and 2 . Then, the differential equations

$$
\nabla^{2} \phi_{i}=0 \text { in fluid } i
$$

govern the potential flow: the conditions at the interface are given by

$$
\left.\frac{\partial \eta}{\partial t}\right|_{y=\eta}-\left.\frac{\partial \phi_{1}}{\partial x} \frac{\partial \eta}{\partial x}\right|_{y=\eta}+\left.\frac{\partial \phi_{1}}{\partial y}\right|_{y=\eta}=0
$$




$$
\begin{gathered}
\left.\frac{\partial \eta}{\partial t}\right|_{y=\eta}-\left.\frac{\partial \phi_{2}}{\partial x} \frac{\partial \eta}{\partial x}\right|_{y=\eta}+\left.\frac{\partial \phi_{2}}{\partial y}\right|_{y=\eta}=0 \\
\left.\rho_{1} \frac{\partial \phi_{1}}{\partial t}\right|_{y=\eta}-\left.\rho_{2} \frac{\partial \phi_{2}}{\partial t}\right|_{y=\eta}+\left.\frac{\rho_{2}}{2}\left[\left(\frac{\partial \phi_{2}}{\partial x}\right)^{2}+\left(\frac{\partial \phi_{2}}{\partial y}\right)^{2}\right]\right|_{y=\eta}-\left.\frac{\rho_{1}}{2}\left[\left(\frac{\partial \phi_{1}}{\partial x}\right)^{2}+\left(\frac{\partial \phi_{1}}{\partial y}\right)^{2}\right]\right|_{y=\eta} \\
=0
\end{gathered}
$$

The initial conditions are

$$
\eta(x, 0)=a_{0}^{+} \cos (k x),\left.\frac{d \eta}{d t}\right|_{t=0}=a_{0}^{+} \sigma \cos (k x),
$$

where

$$
\sigma=A^{+}[u] k
$$

is a constant given by the linear initial growth predicted by the Richtmyer model [see Eq. (11)].

A perturbation solution is assumed, and each term is further expanded into a series as

$$
\begin{aligned}
\eta(x, t) & =\sum_{n=1}^{\infty} \eta^{(n)}(x, t) \\
\phi_{i}(x, y, t) & =\sum_{n=0}^{\infty} \phi_{i}^{(n)}(x, y, t) \\
\eta^{(n)}(x, t) & =\sum_{j=1}^{n} a_{j}^{(n)}(t) \cos (j k x) \\
\phi_{1}^{(n)}(x, y, t) & =\sum_{j=0}^{n} b_{1 j}^{(n)}(t) \cos (j k x) \mathrm{e}^{-j k y} \\
\phi_{2}^{(n)}(x, y, t) & =\sum_{j=0}^{n} b_{2 j}^{(n)}(t) \cos (j k x) \mathrm{e}^{j k y} .
\end{aligned}
$$

Collecting terms of the same order leads to a system of ordinary differential equations in time that can be easily integrated. The first three terms of the solution are given by

$$
\begin{aligned}
\eta^{(1)}(x, t) & =\left(a_{0}^{+}+a_{0}^{+} \sigma t\right) \cos (k x) \\
\eta^{(2)}(x, t) & =\frac{1}{2} k\left(a_{0}^{+}\right)^{2} \sigma^{2} A^{+} t^{2} \cos (2 k x) \\
\eta^{(3)}(x, t) & =-\frac{1}{24} k^{2}\left(a_{0}^{+}\right)^{3} \sigma\left\{\left[4\left(A^{+}\right)^{2}+1\right] \sigma^{2} t^{3}+3 \sigma t^{2}+6 t\right\} \cos (k x) \\
& +\frac{1}{8} k^{2}\left(a_{0}^{+}\right)^{3} \sigma\left\{\left[4\left(A^{+}\right)^{2}-1\right] \sigma^{2} t^{3}-3 \sigma t^{2}\right\} \cos (3 k x)
\end{aligned}
$$

As seen from the expression for $\eta^{(3)}$, additional higher-order terms in the perturbation series becomes quite complicated.

The series approximation can be evaluated at the locations of the spike and bubble to yield the mixing layer width. The bubble and spike are located at $x=0$ and $x=\pi / k$, respectively; thus, the width defined as half the distance between spike and bubble is

$$
a(t)=\frac{1}{2}\left[\eta(0, t)-\eta\left(\frac{\pi}{k}, t\right)\right]
$$


The above formulation yields an independent series for the spike and the bubble. These two separate series can be used to evaluate models for the amplitude of the bubble or spike. The growth of the mixing layer is given by

$$
\frac{\mathrm{d} a}{\mathrm{~d} t}=v_{0}\left\{1-k^{2} v_{0} t a_{0}^{+}+\left[\left(A^{+}\right)^{2}-\frac{1}{2}\right] k^{2} v_{0}^{2} t^{2}\right\},
$$

where $k$ is the wavenumber of the initial perturbation, and $v_{0}$ is the Richtmyer velocity (12). Unfortunately, the range of validity of this finite Taylor series approximation is limited. For this reason, Padé approximations (see below) are used to extend the approximation into the nonlinear regime:

$$
\frac{\mathrm{d} a}{\mathrm{~d} t}=\frac{v_{0}}{1+k^{2} v_{0} a_{0}^{+} t+\max \left[0,\left(k a_{0}^{+}\right)^{2}-\left(A^{+}\right)^{2}+\frac{1}{2}\right] k^{2} v_{0}^{2} t^{2}} .
$$

Thus, the amplitude is given by

$$
\begin{aligned}
& a(t)= \frac{2}{k \sqrt{4 \max \left[0,\left(k a_{0}^{+}\right)^{2}-\left(A^{+}\right)^{2}+\frac{1}{2}\right]-\left(k a_{0}^{+}\right)^{2}}} \\
& \times \tan ^{-1}\left\{\frac{k a_{0}^{+}+2 \max \left[0,\left(k a_{0}^{+}\right)^{2}-\left(A^{+}\right)^{2}+\frac{1}{2}\right] k v_{0} t}{\sqrt{4 \max \left[0,\left(k a_{0}^{+}\right)^{2}-\left(A^{+}\right)^{2}+\frac{1}{2}\right]-\left(k a_{0}^{+}\right)^{2}}}\right\} .
\end{aligned}
$$

The choice of the Padé approximant $P_{2}^{0}$ for $\left(k a_{0}^{+}\right)^{2} \geq\left(A^{+}\right)^{2}-1 / 2$ and $P_{1}^{0}$ for $\left(k a_{0}^{+}\right)^{2} \leq$ $\left(A^{+}\right)^{2}-1 / 2$ matches the asymptotic growth observed experimentally for large $t$. The amplitude growth predicted by the model was in excellent agreement with numerical simulation results and with experimental data conducted in air $/ \mathrm{SF}_{6}$.

To clarify the role of Padé approximations, a brief explanation following Bender and Orszag [11] is presented. Padé approximations arise in the context of summation of perturbation series. It is often the case that only a few terms are available when a perturbation series is formed for a small parameter $\epsilon$. These terms often converge very slowly to the desired solution, or they may diverge due to the presence of a singularity. Padé approximations offer the possibility of improving the behavior of diverging perturbation series based on the knowledge of only a few terms. Padé approximations are also used to improve the convergence properties of diverging power series. In many instances, the regular power series may diverge as it reaches the boundaries of the region of convergence: Padé approximants can further extend the range of validity.

In a Padé approximation, the power series $\sum_{n} a_{n} z^{n}$ is replaced by a sequence of rational functions $P_{M}^{N}$ called the Padé approximants. The indices $M$ and $N$ denote the order of the polynomial in the denominator and in the numerator, respectively. Thus, the Padé approximant can be expressed as

$$
P_{M}^{N}(z)=\frac{\sum_{n=0}^{N} A_{n} z^{n}}{\sum_{n=0}^{M} B_{n} z^{n}}
$$

with coefficients $\left\{A_{n}\right\}_{n=0}^{N}$ and $\left\{B_{n}\right\}_{n=0}^{M}$. Note that, without loss of generality, $B_{0}=1$. The other $M+N+1$ terms are chosen so that the first $M+N+1$ terms in the Taylor series for $P_{M}^{N}$ 
match the $M+N+1$ terms in the series $\sum_{n} a_{n} z^{n}$. As $M$ and $N$ increase, $P_{M}^{N}(z)$ converges even if the series $\sum_{n} a_{n} z^{n}$ diverges or even in regions where the series summation is no longer convergent.

It is straightforward to determine the values of the coefficients of a Padé series given the series $\sum_{n} a_{n} z^{n}$. The coefficients $B_{i}$ satisfy the matrix equation

$$
\begin{aligned}
\mathbf{C}\left[\begin{array}{c}
B_{1} \\
B_{2} \\
\vdots \\
B_{M}
\end{array}\right] & =-\left[\begin{array}{c}
a_{N+1} \\
a_{N+2} \\
\vdots \\
a_{N+M}
\end{array}\right], \\
\mathbf{C}_{i j} & =a_{N+i-j} .
\end{aligned}
$$

The elements of the matrix $\mathbf{C}$ are constant along diagonals. Also note that if the index $N+i-j<0$, then its value can be taken to be zero. Once the $B_{i}$ are determined, the $A_{i}$ are computed as

$$
A_{i}=\sum_{j=0}^{i} a_{i-j} B_{j}
$$

and $B_{j}=0$ for $j>M$.

\subsubsection{The Vandenboomgaerde et al. model}

Vandenboomgaerde, Gauthier, and Mügler [98] proposed a simplified version of the perturbation expansion of Zhang and Sohn [107]. First, choose

$$
\sigma=\frac{k[u]}{2}\left(A^{+}+\frac{A^{-}}{1-\frac{[u]}{u_{\text {shock }}}}\right)
$$

so that $a_{0} \sigma$ gives the right side of Eq. (23). Noting that an accurate perturbation series can be obtained by retaining only the secular terms (i.e., the terms with the largest unbounded part), only the largest power from each term of the Zhang and Sohn solution Eq. (52) must be retained. This yields

$$
\begin{aligned}
& \eta^{(1)}(x, t)=\left(a_{0}^{+}+a_{0}^{+} \sigma t\right) \cos (k x) \\
& \eta^{(2)}(x, t)=\frac{1}{2}\left(a_{0}^{+}\right)^{2} k \sigma^{2} A^{+} t^{2} \cos (2 k x) \\
& \eta^{(3)}(x, t)=-\frac{1}{8} k^{2}\left(a_{0}^{+}\right)^{2} \sigma^{3} t^{3}\left\{\frac{1}{3}\left[4\left(A^{+}\right)^{2}+1\right] \cos (k x)-\left[4\left(A^{+}\right)^{2}-1\right] \cos (3 k x)\right\}
\end{aligned}
$$

Such an approximation is usually valid for large times, but in this case the first two terms of the series are identical to the series of Zhang and Sohn, so that good agreement is expected between the predictions of this model and the Zhang-Sohn model, even at small times. Another advantage of this method is that high-order terms can be easily computed. As only the high-order terms in the series are retained, the determination of the coefficients shifts from integrating in time to solving an algebraic system. Using this method gives the series solution up to eleventh-order for the growth of the mixing layer:

$$
a(t)=a_{0}^{+}+\frac{1}{k} \sum_{n=0}^{5} P_{2 n+1}\left(a_{0}^{+} k \sigma t\right)^{2 n+1},
$$


where

$$
\begin{aligned}
& P_{1}(x)=1 \\
& P_{3}(x)=-\frac{1}{6}\left(1-2 x^{2}\right) \\
& P_{5}(x)=\frac{1}{240}\left(19-125 x^{2}+92 x^{4}\right) \\
& P_{7}(x)=-\frac{1}{5040}\left(264-3686 x^{2}+6997 x^{5}-3234 x^{6}\right) \\
& P_{9}(x)=\frac{1}{2903040}\left(117663-2855274 x^{2}+10086083 x^{4}-11093856 x^{6}+3805728 x^{8}\right) \\
& P_{11}(x)=-\frac{1}{159667200}\left(5507319-206796915 x^{2}+1168865775 x^{4}-2250383605 x^{6}\right. \\
& \left.\quad+1755444326 x^{8}-483163144 x^{10}\right)
\end{aligned}
$$

This series solution diverges at $t \approx\left(a_{0}^{+} k \sigma\right)^{-1}$; therefore, Padé approximants are used to extend the validity of the solution. Note that the Padé approximation is constructed for the amplitude growth rate $\mathrm{d} a / \mathrm{d} t$ and not for the amplitude itself $a(t)$, which yields better results and is also in the spirit of the work of Zhang and Sohn [107]. The growth rate is given by the tenth-degree polynomial

$$
\frac{\mathrm{d} a}{\mathrm{~d} t}=a_{0}^{+} \sigma \sum_{n=0}^{5}(2 n+1) P_{2 n+1}\left(a_{0}^{+} k \sigma t\right)^{2 n} .
$$

A $P_{6}^{4}$ Padé approximant is constructed as

$$
P_{6}^{4}(t)=\frac{\sum_{n=0}^{4} A_{n}\left(a_{0}^{+} k \sigma t\right)^{n}}{\sum_{n=0}^{6} B_{n}^{n}\left(a_{0}^{+} k \sigma t\right)}
$$

with the Padé coefficients $\left\{A_{n}\right\}$ and $\left\{B_{n}\right\}$ computed as described above (the values are not presented as the analytical expressions are complex and can be easily computed).

\subsection{Potential flow models}

Potential flow models can describe the amplitude evolution of the Rayleigh-Taylor and RichtmyerMeshkov instabilities through the nonlinear regime. A potential flow model provides a description of the flow through late times by the velocity evolution of bubbles and spikes. Layzer [60] developed the first potential flow model to describe the Rayleigh-Taylor instability. This model was subsequently extended to the Richtmyer-Meshkov instability.

\subsubsection{The Layzer model for the Rayleigh-Taylor instability}

Layzer [60] presented analytic solutions for the flow observed when an ideal, incompressible fluid contained in the upper half of a vertical tube falls under the action of gravity. In the experiments, the lower half of the tube was empty and the surface was initially flat. Disturbances were applied so that a single "vacuum" bubble rises at the center of the tube. Layzer obtained solutions for the velocity of the bubble tip in the case of a two-dimensional channel and a three-dimensional circular tube. Note that the density ratio of the two fluids in the system above is effectively infinite, corresponding to an Atwood number $A=1$.

For inviscid fluids initially at rest, the velocity field can be described by a scalar potential $\phi(x, y, t)$ and $\phi(x, y, z, t)$ in two and three dimensions, respectively, satisfying the Laplace and Bernoulli equations. The Laplace equation is

$$
\nabla^{2} \phi=0
$$


where $\nabla^{2}=\partial^{2} / \partial x^{2}+\partial^{2} / \partial y^{2}$ in two dimensions and $\nabla^{2}=\partial^{2} / \partial x^{2}+\partial^{2} / \partial y^{2}+\partial^{2} / \partial z^{2}$ in three dimensions. The Bernoulli equations are

$$
\begin{aligned}
& \left.\frac{\partial \phi}{\partial t}\right|_{y=\eta}-\left.\frac{1}{2}\left[\left(\frac{\partial \phi}{\partial x}\right)^{2}+\left(\frac{\partial \phi}{\partial y}\right)^{2}\right]\right|_{y=\eta}-\left.g \eta\right|_{y=\eta}=\text { constant } \\
& \left.\frac{\partial \phi}{\partial t}\right|_{z=\eta}-\left.\frac{1}{2}\left[\left(\frac{\partial \phi}{\partial r}\right)^{2}+\left(\frac{\partial \phi}{\partial z}\right)^{2}\right]\right|_{z=\eta}-\left.g \eta\right|_{z=\eta}=\text { constant }
\end{aligned}
$$

in two and three dimensions, respectively, where the three-dimensional equation is written in polar coordinates. The interface further satisfies the kinematic condition

$$
\begin{aligned}
& \left.\frac{\partial \phi}{\partial z}\right|_{y=\eta}=\left.\frac{\partial \eta}{\partial t}\right|_{y=\eta}+\left.\frac{\partial \phi}{\partial x} \frac{\partial \eta}{\partial x}\right|_{y=\eta}, \\
& \left.\frac{\partial \phi}{\partial z}\right|_{z=\eta}=\left.\frac{\partial \eta}{\partial t}\right|_{z=\eta}+\left.\frac{\partial \phi}{\partial r} \frac{\partial \eta}{\partial r}\right|_{z=\eta},
\end{aligned}
$$

in two and three dimensions, respectively. The ansatz for the perturbation is given by

$$
\begin{aligned}
\phi(x, y, t) & =F(t) \mathrm{e}^{-y} \cos x, \\
\phi(r, z, t) & =F(t) \mathrm{e}^{-z} J_{0}(r)
\end{aligned}
$$

in two and three dimensions, respectively, where $J_{0}(r)$ is the Bessel function of order zero.

Let $g=1$ and define

$$
T(t)=1+\int_{t_{0}}^{t} F(t) \mathrm{d} t
$$

so that $F(t) \equiv \mathrm{d} T(t) / \mathrm{d} t$ and $\eta$ satisfy

$$
\mathrm{e}^{\eta}=T\left[1-\frac{r^{2}}{8}\left(1-\frac{1}{T^{2}}\right)\right] .
$$

Substituting these expressions into the Bernoulli equations gives a differential equation

$$
\begin{gathered}
T\left(T^{2}+1\right) \frac{\mathrm{d}^{2} T}{\mathrm{~d} t^{2}}-\left(\frac{\mathrm{d} T}{\mathrm{~d} t}\right)^{2}-T^{2}\left(T^{2}-1\right)=0 \\
T\left(2 T^{3}+1\right) \frac{\mathrm{d}^{2} T}{\mathrm{~d} t^{2}}-\left(T^{3}-1\right)\left(\frac{\mathrm{d} T}{\mathrm{~d} t}\right)^{2}-T^{2}\left(T^{3}-1\right)=0
\end{gathered}
$$

in two and three dimensions, respectively.

Let $a_{b}^{R T}(t)$ denote the amplitude of the Rayleigh-Taylor bubble, related to $T(t)$ by

$$
a_{b}^{R T}(t)=\log T(t) .
$$

Let $v_{b}^{R T}$ denote the velocity of the bubble. Then, for $a_{b}^{R T}(0)=0$, it follows that Eqs. (76) and (77) can be integrated to give

$$
\begin{aligned}
v_{b}^{R T}(t) & =\frac{\mathrm{d} a_{b}^{R T}}{\mathrm{~d} t} \\
& =\sqrt{\frac{\exp \left(3 a_{b}^{R T}\right)-3 \eta-1}{3\left[\exp \left(3 a_{b}^{R T}\right)+\frac{1}{2}\right]}},
\end{aligned}
$$




$$
\begin{aligned}
v_{b}^{R T}(t) & =\frac{\mathrm{d} a_{b}^{R T}}{\mathrm{~d} t} \\
& =\sqrt{\frac{\exp \left(2 a_{b}^{R T}\right)-2 \eta-1}{\exp \left(2 a_{b}^{R T}\right)+1}}
\end{aligned}
$$

in two and three dimensions, respectively. These equations can be integrated to obtain the position of the bubble tip. For late times, the velocities are given by

$$
\begin{aligned}
v_{b}^{R T} & =\sqrt{\frac{g R}{\beta_{1}}} \\
v_{b}^{R T} & =\sqrt{\frac{g}{3 k}}
\end{aligned}
$$

in two and three dimensions, respectively, where $\beta_{1} \approx 3.832$ is the first zero of the Bessel function of order one.

\subsubsection{The Hecht-Alon-Shvarts model for the Richtmyer-Meshkov instability}

Hecht, Alon, and Shvarts [38] extended the Layzer model to the Richtmyer-Meshkov instability. The two-dimensional equations for the potential $\phi$ are modified so that $g=0$ and an initial velocity perturbation $v_{b}^{R M}(0)$ equal to the change in velocity after the shock is introduced. The velocity at late times is

$$
v_{b}^{R M}(t)=\frac{2}{3 k t},
$$

and is independent of the initial velocity perturbation. The asymptotic bubble curvature is

$$
\kappa=\frac{3 \lambda}{2 \pi}
$$

which is also the same as for Rayleigh-Taylor bubbles. The predictions of this model were in good agreement with numerical simulations for $A=1$.

\subsubsection{The Mikaelian model for arbitrary initial perturbations}

Mikaelian [74] extended the Layzer model to the case when $a_{b}(0) \neq 0$ for both the RayleighTaylor and the Richtmyer-Meshkov instability. Equations for the bubble velocity in the Rayleigh-Taylor and Richtmyer-Meshkov instabilities were derived in two- and three-dimensional geometries. The late-time limit of $v_{b}^{R T}$ in two and three dimensions was shown to be consistent with the values determined by Layzer in Eqs. (82) and (81), respectively. For the RichtmyerMeshkov instability, the late-time two- and three-dimensional bubble velocities are

$$
\begin{aligned}
v_{b}^{R M}(t) & =\frac{2}{3 k t}, \\
v_{b}^{R M}(t) & =\frac{R}{\beta_{1} t},
\end{aligned}
$$

respectively, in agreement with the earlier result of Hecht [38] in Eq. (83). 


\subsubsection{The Zhang model for the velocity of spikes}

Zhang [106] extended the Layzer model to determine the velocity of spikes for both the Richtmyer-Meshkov and the Rayleigh-Taylor instabilities in two and three dimensions. Expressions for the spike and bubble velocity were determined by assuming that the interface is locally parabolic,

$$
\eta(y, t)=a(t)+\xi(t) k y^{2} .
$$

Substitution into the governing equations gives a system of ordinary differential equations for $a(t)$ and $\xi(t)$ in terms of $F(t)$ from Eq. (72):

$$
\begin{gathered}
\frac{\mathrm{d} a}{\mathrm{~d} t}=F k \mathrm{e}^{-k a}, \\
\frac{\mathrm{d} \xi}{\mathrm{d} t}=-F k^{2}\left(3 \xi+\frac{1}{2}\right) \mathrm{e}^{-k a}, \\
k \mathrm{e}^{-k a}\left(\xi+\frac{1}{2}\right) \frac{\mathrm{d} F}{\mathrm{~d} t}=-F^{2} k^{3} \xi \mathrm{e}^{-2 k a}-g \xi .
\end{gathered}
$$

It is possible to eliminate $F(t)$ from the first two equations to obtain

$$
\xi(a(t))=\left\{\left[\xi(0)+\frac{1}{6}\right] \mathrm{e}^{-3 k[a-a(0)]}-\frac{1}{6}\right\}
$$

and a differential equation for $v(t)=\mathrm{d} a(t) / \mathrm{d} t=v(\xi(t))$ :

$$
-\frac{k^{2}}{4}(6 \xi+1) \frac{\mathrm{d} v^{2}}{\mathrm{~d} \xi}+\frac{k^{2}}{2 \xi+1} v^{2}+\frac{2 \xi}{2 \xi+1} g=0 .
$$

The differential equation (92) can be solved for $v=v(\xi(t))$. It is then possible to obtain an expression $v=v(a(t))$ via the substitution $\xi=\xi(a(t))$ in Eq. (91) to obtain the velocity for the bubble and spike

$$
v=\sqrt{\frac{9[2 \xi(0)+1] k v(0)^{2}-6[6 \xi(0)+1][a-a(0)] k g+2\left(\mathrm{e}^{3 k[z-z(0)]}-1\right) g}{3 k\left[6 \xi(0)+1+2 \mathrm{e}^{3 k[a-a(0)]}\right]}} .
$$

In the case of a sinusoidal initial perturbation, $\xi(0)=-a(0) k / 2$ for the bubble and $\xi(0)=$ $a(0) k / 2$ for the spike. The late-time asymptotic solutions are

$$
\begin{array}{cccc}
v_{b}^{R T} \longrightarrow \sqrt{\frac{g}{3 k}} & \xi_{b}^{R T} & \longrightarrow & -\frac{1}{6} \\
v_{s}^{R T}(t) \longrightarrow-g t & \xi_{s}^{R T} & \longrightarrow \infty \\
v_{b}^{R M}(t) \longrightarrow \frac{2}{3 k t} & \xi_{b}^{R M} & \longrightarrow & -\frac{1}{6} \\
v_{s}^{R M} \longrightarrow v_{0} \sqrt{\frac{6 \xi_{0}+3}{6 \xi_{0}+1}} & \xi_{s}^{R M} & \longrightarrow & \infty,
\end{array}
$$

where $b$ and $s$ denote the bubble and spike, respectively. The novel components of this model are the spike equations (95) and (97). The equations for the bubble in two dimensions, (94) and (96), were previously derived by Layzer [60] in Eq. (82) and by Hecht [38] in Eq. (85). The model predicts constant linear acceleration for a spike in the Rayleigh-Taylor instability and constant velocity in the Richtmyer-Meshkov instability (depending on the initial conditions $v_{0}$ and $\left.\xi_{0}\right)$. The solutions are consistent with the results of numerical computations based on conformal mapping [68] and on finite-differencing [2]. 


\subsubsection{The Goncharov model for arbitrary Atwood numbers}

Goncharov [35] extended the two-dimensional Layzer model to the case of $A \neq 1$ for both the Rayleigh-Taylor and the Richtmyer-Meshkov instability. The Bernoulli equation is modified to include the density and is given at $y=\eta$ by

$$
\begin{aligned}
\left\{\rho_{1} \frac{\partial \phi_{1}}{\partial t}+\frac{\rho_{1}}{2}\left[\left(\frac{\partial \phi_{1}}{\partial x}\right)^{2}+\right.\right. & \left.\left.\left(\frac{\partial \phi_{1}}{\partial y}\right)^{2}\right]+\rho_{1} g y\right\} \\
& -\left\{\rho_{2} \frac{\partial \phi_{2}}{\partial t}+\frac{\rho_{2}}{2}\left[\left(\frac{\partial \phi_{2}}{\partial x}\right)^{2}+\left(\frac{\partial \phi_{2}}{\partial y}\right)^{2}\right]+\rho_{2} g y\right\}=f(t),
\end{aligned}
$$

where $f(t)$ is an arbitrary function, and $\eta(t)$ is given by Eq. (87). The velocity potentials for the two fluids $\phi_{1}$ and $\phi_{2}$ assume the form

$$
\begin{gathered}
\phi_{1}(x, y, t)=a_{1}(t) \cos (k x) \mathrm{e}^{-k\left(y-\eta_{0}\right)}, \\
\phi_{2}(x, y, t)=b_{1}(t) \cos (k x) \mathrm{e}^{k\left(y-\eta_{0}\right)}+b_{2}(t) y,
\end{gathered}
$$

where $a_{1}, b_{1}$ and $b_{2}$ are unknown functions. The form of the potential is dictated by the boundary conditions. The equations are again expanded around (87) and solved. Note that $\xi$ is related to the curvature by $\kappa=-1 /(2 \xi)$. The final results for the bubble velocities when $A \neq 1$ are

$$
\begin{gathered}
v_{b}^{R T} \longrightarrow \sqrt{\frac{2 A g}{3(1+A) k}}, \xi_{b}^{R T} \longrightarrow-\frac{k}{6}, \\
v_{b}^{R M}(t) \longrightarrow \frac{3+A}{3(1+A) k t}, \xi_{b}^{R M} \longrightarrow-\frac{k}{6} .
\end{gathered}
$$

\subsubsection{The Sohn model}

Sohn [94] also extended the Layzer model to fluids with arbitrary density ratio. The approach differs from the Goncharov model in the use of a simpler form for the potential functions from Layzer [60] in Eq. (72). The shape of the interface near a bubble tip is assumed to be parabolic [see Eq. (87)]. Substituting this form and expanding yields the system of equations of Zhang, Eqs. (88)-(90), with Eq. (90) now modified to include the Atwood number:

$$
k \mathrm{e}^{-k a}\left(\xi+\frac{1}{2}\right) \frac{\mathrm{d} F}{\mathrm{~d} t}=-A F^{2} k^{3} \xi \mathrm{e}^{-2 k a}-A g \xi .
$$

The asymptotic bubble velocities are determined by solving the evolution equations to obtain

$$
\begin{aligned}
v_{b}^{R T} & \longrightarrow \sqrt{\frac{A g}{(2+A) k}}, \xi_{b}^{R T} \longrightarrow-\frac{k}{6}, \\
v_{b}^{R M}(t) & \longrightarrow \frac{2}{(2+A) k t}, \xi_{b}^{R M} \longrightarrow-\frac{k}{6} .
\end{aligned}
$$

Thus, this model predicts that the velocity of a bubble in a Richtmyer-Meshkov flow decays to zero at asymptotic times. The predictions of this model were validated against numerical simulations in two dimensions. 


\subsubsection{The Sadot et al. empirical model for the Richtmyer-Meshkov instability}

The Sadot et al. [80] empirical model for the Richtmyer-Meshkov instability is based on fits to experimental data and on asymptotic growth laws. The model was presented in the context of providing a single formula that could capture the initial linear growth, as well as the later nonlinear growth for both the bubbles and the spikes. Let $a_{b}(t)$ and $a_{s}(t)$ denote the amplitudes of the bubbles and spikes, and let $v_{0}=k A^{+}[u] a_{0}$ be the Richtmyer velocity, but using the postshock Atwood number. The Sadot model for the velocities of the bubble, spike, and mixing layer is

$$
\begin{gathered}
\frac{\mathrm{d} a_{b}}{\mathrm{~d} t}=\frac{v_{0}\left(1+k v_{0} t\right)}{1+\left(1+A^{+}\right) k v_{0} t+\frac{1}{2 \pi C} k^{2} v_{0}^{2} t^{2}}, \\
\frac{\mathrm{d} a_{s}}{\mathrm{~d} t}=\frac{v_{0}\left(1+k v_{0} t\right)}{1+\left(1-A^{+}\right) k v_{0} t+\frac{1-A^{+}}{1+A^{+}} \frac{1}{2 \pi C} k^{2} v_{0}^{2} t^{2}}, \\
\frac{\mathrm{d} a}{\mathrm{~d} t}=\frac{1}{2}\left(\frac{\mathrm{d} a_{b}}{\mathrm{~d} t}+\frac{\mathrm{d} a_{s}}{\mathrm{~d} t}\right) .
\end{gathered}
$$

The value of $C$ is determined using experimental data, and it is found that for $A^{+} \geq 0.5$, $C=1 /(3 \pi)$. In the limit $A^{+} \rightarrow 0, C=1 /(2 \pi)$. For intermediate values of the Atwood number, the value of $C$ is poorly-determined. The bubble and spike amplitudes corresponding to these growth rates are

$$
\begin{gathered}
a_{b}(t)=a_{b}(0)+\frac{2-2 \pi C\left(1+A^{+}\right)}{k \sqrt{\frac{2}{\pi C}-\left(1+A^{+}\right)^{2}}} \tan ^{-1}\left[\frac{1+A^{+}+\frac{k v_{0} t}{\pi C}}{\sqrt{\frac{2}{\pi C}-\left(1+A^{+}\right)^{2}}}\right] \\
+\frac{\pi C}{k} \ln \left[1+\left(1+A^{+}\right) k v_{0} t+\frac{\left(k v_{0} t\right)^{2}}{2 \pi C}\right], \\
a_{s}(t)=a_{s}(0)+\frac{2-2 \pi C\left(1+A^{+}\right)}{k \sqrt{\frac{2}{\pi C} \frac{1-A^{+}}{1+A^{+}}-\left(1-A^{+}\right)^{2}}} \tan ^{-1}\left[\frac{1-A^{+}+\frac{1-A^{+}}{1+A^{+}} \frac{k v_{0} t}{\pi C}}{\sqrt{\frac{2}{\pi C} \frac{1-A^{+}}{1+A^{+}}-\left(1-A^{+}\right)^{2}}}\right] \\
+\frac{\pi C}{k} \frac{1+A^{+}}{1-A^{+}} \ln \left[1+\left(1-A^{+}\right) k v_{0} t+\frac{1-A^{+}}{1+A^{+}} \frac{\left(k v_{0} t\right)^{2}}{2 \pi C}\right] .
\end{gathered}
$$

This model has been extensively tested against new and previous experimental data, and excellent agreement was found for both the spike and bubble growth. Furthermore, the model appears to be valid over the range $M a=1.3-3.5$. As the model is mainly based on incompressible flow considerations, the authors conclude that compressibility effects are not significant in modeling the growth of the mixing layer.

\subsection{Power-law models for multi-mode initial perturbations and following reshock}

Scaling laws for the mixing layer width arising from a multi-mode initial interfacial perturbation and following reshock of an evolving interface are presented here. In both cases, the shockinterface interaction generates a complex and possibly turbulent mixing layer at late times. The determination of the late-time or asymptotic scaling laws for the growth of the mixing 
layer in the Richtmyer-Meshkov instability remains an open question, and is not considered in the present work.

Multi-mode initial perturbations are particularly relevant, as such perturbations are typically found in nature. The effects of reshock, occurring as the interface undergoes multiple impulsive accelerations by waves, are also of great interest. Reshock occurs in a shock tube experiment when the transmitted shock following the initial interaction with the interface reflects from the end wall of the test section and interacts with the evolving interface. Following reshock, a transmitted shock continues in the first fluid and a reflected rarefaction wave returns into the second fluid. The reflected rarefaction wave reflects from the end wall and interacts again with the evolving interface, generating a reflected compression wave. The reflected compression wave reflects from the end wall and again interacts with the evolving interface. Eventually, the interface comes to rest following a sufficient number of such interactions. Each interaction with a reflected wave deposits additional baroclinic vorticity on the complex evolving interface, and imparts additional energy into the mixing layer. The amount of energy deposited depends on the characteristic of the small scales: for this reason, different growth characteristics are expected in the reshocked, single-mode Richtmyer-Meshkov instability than in the multi-mode Richtmyer-Meshkov instability without reshock.

\subsubsection{The Alon et al. bubble merger model for $A=1$}

Alon et al. [1] developed a statistical bubble merger model for the late-time evolution of a two-dimensional Richtmyer-Meshkov bubble front in the limit $A=1$ corresponding to fluids with very large density ratios; the choice of the Atwood number allows the use of potential theory to model the flow. The use of bubbles is justified by the observation that the late-time evolution of a Richtmyer-Meshkov unstable interface can be modeled by the rise and merger of large bubbles.

The bubbles are characterized by their diameter or wavelength $\lambda_{i}$ and are initially arranged along a line with some distribution for $\lambda$ given by $g(\lambda)$. The bubbles begin rising at velocities

$$
v_{b}\left(\lambda_{i}\right)=\frac{\lambda_{i}}{3 \pi t}
$$

and two adjacent bubbles merge at the rate $\omega\left(\lambda_{i}, \lambda_{i+1}\right)$, giving rise to a new bubble of diameter $\lambda_{i}+\lambda_{i+1}$ with velocity $v_{b}\left(\lambda_{i}+\lambda_{i+1}\right)$. The amplitude of the interface $a_{b}(t)$ is obtained using the average of bubble velocities,

$$
\frac{\mathrm{d} a_{b}}{\mathrm{~d} t}=\left\langle v_{b}\right\rangle .
$$

As observed earlier [3], the dynamics eventually reach a scale-invariant regime in which the distribution of the bubble sizes scales with the average bubble size. In this regime, the mean velocity $\left\langle v_{b}\right\rangle$, the average wavelength $\langle\lambda\rangle$, and the parameter $\theta_{b}$ satisfy the relations

$$
\begin{gathered}
\left\langle v_{b}\right\rangle=\frac{\langle\lambda\rangle}{3 \pi t}, \\
\frac{\mathrm{d}\langle\lambda\rangle}{\mathrm{d} t}=\frac{\theta_{b}\langle\lambda\rangle}{t}, \\
\theta_{b}=\int_{0}^{\infty} \int_{0}^{\infty} \varpi_{0}\left(\frac{x}{y}\right) f(x) f(y) \mathrm{d} x \mathrm{~d} y,
\end{gathered}
$$


where $\varpi_{0}(x / y)$ is the dimensionless merger rate and $f(x)$ is the scaled distribution function that is independent of the initial distribution of bubbles. These yield the power-law scaling

$$
a_{b}(t) \sim a\left(\lambda_{0}, \theta_{b}\right)\left(\frac{t}{t_{0}}\right)^{\theta_{b}}, a\left(\lambda_{0}, \theta_{b}\right)=\frac{\lambda_{0}}{3 \pi \theta_{b}}
$$

for the height of the bubble front in Richtmyer-Meshkov unstable flows, where $t_{0}$ is an arbitrary time in the scale-invariant regime, $\lambda_{0}$ is the average wavelength at that time, and $\theta_{b}=0.4$. This scaling can also be related to the initial conditions (see [2]) by

$$
a_{b}(t)=\frac{c_{B} \bar{\lambda}_{0}}{\theta_{b} \eta^{\theta_{b}}}\left[\frac{\bar{v}_{b}(0) t}{\bar{\lambda}_{0}}\right]^{\theta_{b}},
$$

where $\bar{\lambda}_{0}$ and $\bar{v}_{b}(0)$ are the average initial wavelength and velocity of the bubble, $\eta=O(1)$ is a parameter depending on the initial spectrum, and $c_{B}$ is the scaling constant in the scaleinvariant regime with $\left\langle v_{b}\right\rangle=c_{B}\langle\lambda\rangle / t$. Comparison with numerical simulations showed good agreement with the model prediction.

The model was later extended to arbitrary density ratios [2] by assuming that the merger rate $\varpi$ of Eq. (114) is insensitive to Atwood number variations. Therefore, the scaling law with $\theta_{b}=0.4$ does not change for smaller Atwood numbers. A scaling law was also derived for the amplitude of the spikes

$$
a_{s}(t) \sim \bar{\lambda}_{0}\left(\frac{\bar{u}_{0} t}{\bar{\lambda}_{0}}\right)^{\theta_{s}(A)}
$$

where

$$
\theta_{s}(A)=1-\beta(A)\left(1-\theta_{b}\right)
$$

depends on the Atwood number. The sum $h=a_{b}+a_{s}$ gives the total mixing layer width corresponding to a multi-mode Richtmyer-Meshkov instability at all Atwood numbers.

\subsubsection{The Rikanati et al. vortex model for bubble merger when $A \rightarrow 0$}

Rikanati, Alon and Shvarts [89] reconsidered the modeling of the bubble merger process in the limit of $A \rightarrow 0$. Noting that the potential flow model (which is the basis of the Alon et al. [1] model) is no longer appropriate at small Atwood numbers, an alternative model based on the Jacobs and Sheeley [47] vortex model was proposed, as vortices form shortly after the passage of the shock.

Consider an initial array of identical vortices forming a vortex line, for which the complexvalued potential is

$$
\begin{aligned}
w(z) & =\frac{i \Gamma}{2 \pi} \sum_{n=-\infty}^{\infty} \ln \left(z-n z_{0}\right) \\
& =\frac{i \Gamma}{2 \pi} \ln \left[\sin \left(\frac{\pi z}{z_{0}}\right)\right],
\end{aligned}
$$

where $z_{0}$ is the constant separation between the vortices and $\Gamma$ is the vortex circulation. The single-mode Richtmyer-Meshkov instability can be modeled by two periodic arrays with vortices of opposite strength adjacent to one other. When the initial perturbation is sinusoidal, it is possible to obtain an expression for $\Gamma=a_{0} / k$, which yields

$$
h(t)=\frac{1}{k} \sinh ^{-1}\left(\frac{\Gamma k^{2}}{2 \pi t}\right)
$$


for the amplitude of the mixing layer. Thus, the asymptotic velocity scales as

$$
v_{b}(t)=\frac{1}{2 \pi} \frac{\lambda}{t}
$$

compared to the asymptotic velocity in the case $A=0$ [38] based on the potential model,

$$
v_{b}(t)=\frac{1}{3 \pi} \frac{\lambda}{t}
$$

The difference between the two values is due to the added mass in the case $A=0$.

Now consider the case of a periodic array of bubbles with two different strengths. The merger rate was then approximated as

$$
\omega\left(\frac{\lambda_{1}}{\lambda_{2}}\right)=\frac{1}{\Delta t_{m}}
$$

where $t_{m}$ is the merger time - the time required for the tip of a small bubble to go from positive to negative. This result for $\omega$ and the resulting asymptotic velocity for the single bubble in Eq. (121) are used in the bubble merger model, which yields the scaling law for the bubble amplitude in Eq. (116) with $\theta_{b}=0.4$. Note that the value of $\theta_{b}$ is the same for $A=0$ and $A=1$, further confirming the observation of [2].

\subsubsection{The Prasad et al. late-time model}

Prasad et al. [84] performed experiments to study the late-time evolution of the single-mode Richtmyer-Meshkov instability and developed an empirical power-law model based on their data. At late times, the mixing layer width was assumed to scale according a power-law

$$
h(t)=c t^{\theta}
$$

Experiments were conducted for a $M a=1.55$ shock refracting at a perturbed air $/ \mathrm{SF}_{6}$ interface with different initial amplitudes and wavelengths. The width of the interface was measured up to 32 initial perturbation wavelengths from the initial location to determine the power-law that best fits the data. The scatter from different measurements with different initial amplitudes and wavenumbers is reduced if the data is normalized by the wavenumber $k$. A composite least-squares fit to the data yielded

$$
\begin{aligned}
k h & =0.96(k x)^{0.33} \\
& =0.96(k[u] t)^{0.33}
\end{aligned}
$$

with $x=[u] t$. In a separate study, the empirical scaling

$$
\begin{aligned}
h & =2.43(\lambda x)^{0.26} \\
& =2.43(\lambda[u] t)^{0.26}
\end{aligned}
$$

was shown to minimize the scatter of the data and to give the best fit. 


\subsubsection{The Youngs model for multi-mode initial conditions}

Youngs [103] proposed a model for the late-time scaling of the mixing layer width emerging from the multi-mode Richtmyer-Meshkov instability. Youngs considered random initial amplitudes $a_{0}$ chosen from a Gaussian distribution. The standard deviation in the region $k<k_{\max }$ is flat, where

$$
k_{\max }=\frac{\pi}{\Delta y}
$$

is the Nyquist wavenumber. Therefore, the relation

$$
\sigma^{2}=C \int_{0}^{k_{\max }} \mathrm{d} k
$$

between the standard deviation $\sigma$ and the integral of the wavenumbers $k$ was obtained in two dimensions. For a single-mode Richtmyer-Meshkov instability, $\sigma^{2}=a_{0}^{2} / 2$. The random initial kinetic energy is

$$
K_{0}=\frac{1}{3}\left(\rho_{1}+\rho_{2}\right) k_{\max }(\sigma A[u])^{2} .
$$

Most of the initial kinetic energy arises from modes with wavenumbers near $k_{\text {max }}$ corresponding to an initial wavelength $\lambda_{\text {min }}$. A turbulent layer is assumed to develop when the mixing layer width becomes of order $\lambda_{\text {min }}$. This layer was shown to have width evolving as $t^{\theta}$ with $\theta<1$. The following model was developed to explain this.

Assuming that the velocity field is characterized by a lengthscale $L$ and magnitude $V$, the kinetic energy dissipation rate scales as $\epsilon \sim V^{3} / L$. The model equations are

$$
\begin{gathered}
\frac{\mathrm{d}}{\mathrm{d} t}\left(L V^{2}\right)=-a V^{3}, \\
\frac{\mathrm{d} W}{\mathrm{~d} t}=V, \\
L=b W+c \lambda_{\min },
\end{gathered}
$$

where $a, b$, and $c$ are model parameters, and $W$ is an integral lengthscale [4]. This lengthscale is defined in terms of the averaged volume fractions $\left\langle f_{1}\right\rangle$ and $\left\langle f_{2}\right\rangle$ according to

$$
W(t)=\int_{a_{s}(t)}^{a_{b}(t)}\left\langle f_{1}\right\rangle\left\langle f_{2}\right\rangle \mathrm{d} x,
$$

where the angle brackets denote a spatial average over the periodic direction $y$ (see $\S 4.1$ ). This lengthscale provides a measure of the diffusive width of the mixing layer, and is less sensitive to statistical fluctuations than $h$. The volume fractions of the two fluids $f_{r}$ are defined in terms of their mass fractions $m_{r}(x, y, t)$ by [103]

$$
f_{r}(x, y, t)=\frac{\frac{m_{r}(x, y, t)}{\rho_{r}}}{\frac{m_{1}(x, y, t)}{\rho_{1}}+\frac{m_{2}(x, y, t)}{\rho_{2}}},
$$

such that $f_{1}+f_{2}=1$. Note that $W$ provides an alternative measure of the mixing layer width. The initial value of $V$ is given by

$$
V_{0}=\left(\frac{\mathrm{d} W}{\mathrm{~d} t}\right)_{t=0}=c \sqrt{\left\langle u_{0}^{2}\right\rangle}
$$




$$
\begin{gathered}
c=\frac{1}{\sqrt{2}} \int_{0}^{\infty}[1-\operatorname{erf}(s)] \mathrm{d} s=0.564, \\
\sqrt{\left\langle u_{0}^{2}\right\rangle}=\frac{k_{\max } \sigma U}{\sqrt{2}}|A| .
\end{gathered}
$$

Therefore, for initial conditions $W(0)=0$ and $V(0)=V_{0}$, the power-law solution

$$
W(t)=\frac{c}{b} \lambda_{\min }\left[\left(1+\frac{V_{0} t b}{\theta a \lambda_{\min }}\right)^{\theta}-1\right]
$$

is obtained, where

$$
\theta=\frac{2}{3+a b}
$$

For the case when no kinetic energy dissipation occurs (corresponding to $a=0$ ), the $\theta=2 / 3$ result of Barenblatt [10] is recovered. When kinetic energy dissipation is present, the equations predict $\theta<2 / 3$.

Youngs compared the predictions of the model (138) with the growth rates obtained from multi-mode simulations in two and three dimensions. Initially, the growth rate of $W / \lambda_{\min }$ was the same in two and three dimensions. At intermediate times, $W / \lambda_{\min }$ was larger for the three-dimensional simulations, indicating that mixing is more rapid in three dimensions. At later times, the larger dissipation of kinetic energy in three dimensions causes the value of $W / \lambda_{\min }$ to become smaller than that observed in two-dimensional simulations. For late times, the three-dimensional data was fitted to the model equation with $\theta=0.30$.

\subsubsection{The Mikaelian model for a reshocked interface}

A linear power-law model for the mixing layer width following reshock was developed by Mikaelian [71] based on the experimental results of Read [86] and Youngs [101] for the the width of the mixing layer of a Rayleigh-Taylor mixing layer

$$
h(t)=0.14 A g t^{2},
$$

where $A$ is the Atwood number and $g$ is the acceleration. Differentiating this expression twice, taking $g=[u]_{1} \delta(t)$ (where $[u]_{1}$ is the change in velocity resulting from the reshock), and integrating twice gives (assuming no dependence on initial conditions and neglecting molecular dissipation effects) [71]

$$
h(t)=0.28[u]_{1} A_{1}^{+} t
$$

where $A_{1}^{+}$is the post-reshock Atwood number.

\subsubsection{The Brouillette-Sturtevant model for reshocked interfaces}

Brouillette and Sturtevant [14] performed shock tube experiments to measure the effect of a thick, diffuse interface on the growth of the Richtmyer-Meshkov instability. In these experiments, a thin metal plate initially separated air and $\mathrm{SF}_{6}$ or air and Freon-22 gas. Prior to launching the shock wave in air, the plate was slowly withdrawn to generate a quasi-sinusoidal perturbation, leaving a diffuse, thick interface separating the two gases. The shock launched in air at Mach numbers 1.12-1.66 refracted at the interface and was transmitted into the second

gas. The transmitted shock reflected from the end wall of the shock tube test section and reshocked the interface. 
The growth of the width of a single-mode interface following $N+1$ impulsive accelerations was empirically modeled by generalizing the Richtmyer model:

$$
\left(\frac{\mathrm{d} h}{\mathrm{~d} t}\right)_{N}=2 k \sum_{i=0}^{N} \frac{[u]_{i} A_{i}^{+} a_{i}^{+}}{\psi_{i}^{+}}
$$

where $k$ is the initial perturbation wavenumber, $[u]_{i}$ is the change in the velocity of the interface corresponding to the ith wave, $A_{i}^{+}$and $a_{i}^{+}$are the post-shock Atwood number and interface width, $\psi_{i}^{+}$is the post-shock growth reduction factor of Duff et al. [32] (see $\S 2.1 .5$ ), and the factor of 2 accounts for the mixing layer width (which is twice the mixing layer amplitude a). As the growth is based on the Richtmyer model for each impulsive acceleration, the Brouillette-Sturtevant model predicts piecewise-linear in time amplitude growth phases. This model derived for shocks can also be used for reflected rarefaction waves and, thus, constitutes an appropriate model for the description of the reshock phase, as it accounts for the multiple waves that successively interact with the interface. The reflected shock refracts at the evolving interface during reshock to produce an expansion wave. This expansion wave reflects from the end wall of the shock tube and interacts with the interface, producing a reflected compression wave. The compression wave reflects from the end wall and interacts again with the interface, and this process continues. Brouillette and Sturtevant reported good agreement between their experimental data and the prediction of Eq. (142).

\subsection{The Samtaney-Zabusky-Ray model for baroclinic circulation deposition}

Samtaney and Zabusky [92] and Samtaney, Ray and Zabusky [91, 85] derived analytical scaling laws for the circulation $\Gamma$ per unit unshocked length deposited on a planar interface by a shock. The interaction can be classified as fast/slow or slow/fast based on the relative speed of the incident and transmitted shocks. Fast/slow interactions occur when the refraction is from a lighter fluid into a heavier fluid and is associated with a reflected shock wave. Slow/fast interactions occur when the refraction is from a heavier fluid to a lighter fluid and is usually associated with a reflected rarefaction wave. In the present investigation, a slow/fast interaction is observed for the initial shock refraction from air(acetone) into $\mathrm{SF}_{6}$ and a fast/slow interaction is observed at reshock.

The circulation deposition model is derived from shock polar analysis (see [57]). In shock refraction, five regions can be identified when the flow is considered in a frame moving with the triple-point, as shown in Fig. 5. The parameters of the model are the density ratio $r \equiv \rho_{1} / \rho_{2}$, the shock/interface angle $\alpha$, the shock Mach number $M a$, and the adiabatic exponents of the two gases $\gamma_{r}$. For scaling purposes, the adiabatic exponents are taken to be equal.

In the case of a fast/slow interaction in the limit of a strong shock $M a \rightarrow \infty$, the circulation deposition $\Gamma$ is given by

$$
\frac{\Gamma}{M a} \longrightarrow \frac{\sqrt{\gamma}}{\sin \alpha}\left(\sqrt{1-\frac{2 p_{\infty} \sin ^{2} \alpha}{(\gamma+1) r}}-\sqrt{1-\frac{2\left(1+\mu^{2} p_{\infty}\right) \sin ^{2} \alpha}{(\gamma+1)\left(\frac{1}{\mu^{2}}+\frac{1+\mu^{2}}{p_{\infty}}\right)}}\right) \frac{\cos \alpha}{\cos \left(\alpha-\delta_{b \infty}\right)},
$$

where $\mu \equiv(\gamma-1) /(\gamma+1), p_{\infty} \equiv p_{5} / M a^{2}$, and $\delta_{b \infty}$ represents the asymptotic flow turning angle in region 2 as $M a \rightarrow \infty$.

For weak shocks with $M a \rightarrow 1$,

$$
\Gamma=\frac{2 \sqrt{\gamma}}{\gamma+1} \frac{\sqrt{r}-1}{\sqrt{r}} \sin \alpha\left(1+\frac{1}{M a}+\frac{2}{M a^{2}}\right)(M a-1) .
$$


For small density discontinuities across the interface, the circulation is expressed as a series in $r^{\prime}=1-1 / \sqrt{r}$.

The circulation scaling law proposed is

$$
\Gamma=\frac{2 \sqrt{\gamma}}{\gamma+1}\left(1-\frac{1}{\sqrt{\gamma}}\right) \sin \alpha\left(1+\frac{1}{M a}+\frac{2}{M a^{2}}\right)(M a-1) .
$$

In the case of a slow/fast interaction and for strong shocks $(M a \rightarrow \infty)$,

$$
\Gamma \longrightarrow \frac{K(r, \alpha, \gamma)}{\sqrt{1-\xi(M a)}},
$$

where

$$
\begin{gathered}
K(r, \alpha, \gamma)=\frac{\sqrt{\gamma+1} \cot \alpha}{\sqrt{2} \cos \left(\alpha-\delta_{b}\right)} \\
\times\left(\sqrt{1-\frac{1}{r} \frac{4 \gamma p^{(\gamma-1) / \gamma} \sin ^{2} \alpha}{\left(1+\mu^{2}\right)^{(\gamma-1) / \gamma}(\gamma+1)^{2}}}-\sqrt{1-\frac{4 \gamma p^{(\gamma-1) / \gamma} \sin ^{2} \alpha}{\left(1+\mu^{2}\right)^{(\gamma-1) / \gamma}\left[(\gamma+1)^{2}-4 \gamma \sin ^{2} \alpha\right]}}\right),
\end{gathered}
$$

and

$$
\xi(M a)=\frac{\frac{2 \gamma}{\gamma+1}\left(M a^{2}-1\right)}{1+\frac{2 \gamma}{\gamma+1}\left(M a^{2}-1\right)}
$$

is the normalized pressure jump.

In the limit of weak shocks with $M a \rightarrow 1$,

$$
\Gamma=\frac{2}{\sqrt{\gamma}}\left(1-\frac{1}{\sqrt{r}}\right) \xi(M a) \sin \alpha .
$$




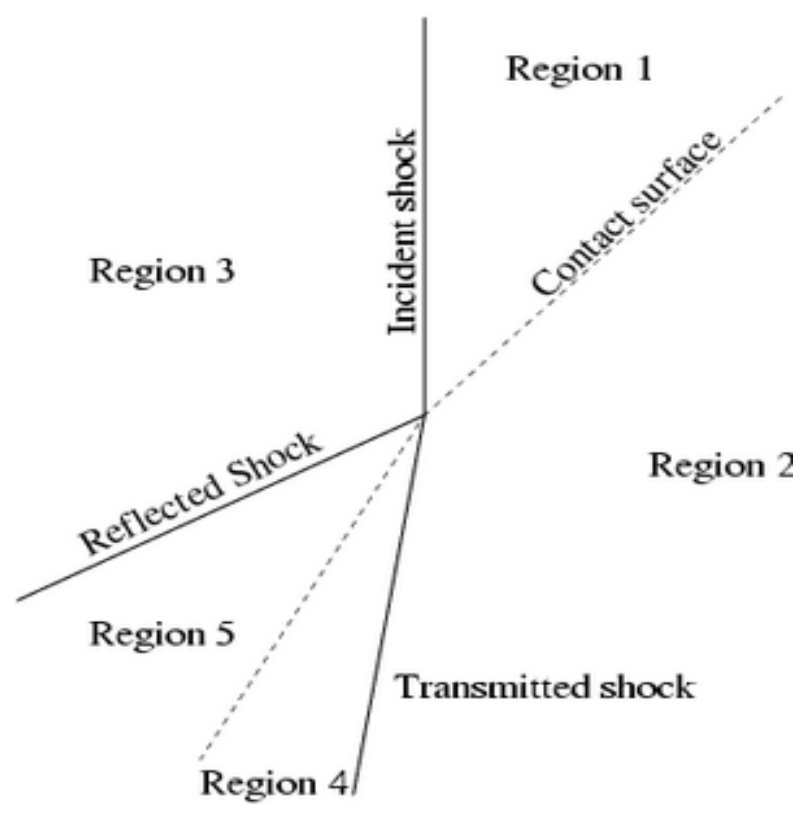

Figure 5: The wave structure of a shock interacting with a contact surface in a reference frame moving with the point of contact. Note the reflected and the transmitted shocks. 


\section{Numerical simulations of the single-mode Richtmyer-Meshkov instability with reshock}

In this section, the HOPE code based on the formally high-order accurate weighted essentially non-oscillatory (WENO) shock-capturing method is used to simulate the two-dimensional, single-mode Richtmyer-Meshkov instability with reshock (see Part 1 of this report [57] for a detailed discussion of the WENO method and a description of the HOPE code). A third-order TVD Runge-Kutta time-evolution scheme was used with a CFL number of 0.75 . The WENO method is a modern, high-resolution reconstruction-evolution method for shock-capturing [55, 62]. As such, the numerical algorithm based on the discretization of the equations contains implicit truncation errors that can be interpreted as a nonlinear numerical dissipation. Hence, the present simulations can be interpreted as a class of monotone integrated large-eddy simulations (MILES) [12, 30, 31], in which the discrete equations are implicitly filtered and the implicit numerical dissipation is a surrogate for a dissipation provided by an explicit subgrid-scale model. As the non-dissipative compressible fluid dynamics equations are formally ill-posed [21], this numerical dissipation regularizes the method and renders it numerically stable for increasingly fine grids. Formally higher-order reconstructions are less dissipative than lower-order reconstructions. MILES methods typically dissipate velocity and scalar fluctuations approximately in the same manner numerically. Thus, the numerical Schmidt number is of $O(1)$, which may provide a reasonable approximate model for the mixing of gases. However, this approximation is clearly not valid for the case for fluids with large (molecular) Schmidt numbers. All of the numerical results presented in this section were obtained using the fifth-order WENO method with a grid resolution $\Delta x=\Delta y$ corresponding to 256 points per initial perturbation wavelength. It should be noted that the present work should not be regarded as a complete endorsement of the WENO method; rather, this work should be regarded as a preliminary investigation of the properties of this method as applied to the Richtmyer-Meshkov instability with reshock. Ultimately, the WENO method should be further modified to reduce the numerical dissipation and improve its resolving power: the hybridization of the WENO method with a central difference scheme, based on multi-resolution analysis, represents one such approach (see [57]).

It should be noted that the WENO method has been previously applied to the RichtmyerMeshkov instability. Kremeyer et al. [54] used a fifth-order WENO method with a thirdorder TVD Runge-Kutta time-evolution scheme to perform two-dimensional simulations of the Richtmyer-Meshkov instability evolution in a shock tube containing gases with different initial transverse density profiles to investigate shock splitting and, in particular, the role of shock bowing and vorticity dynamics. Top-hat shaped perturbations, including those shaped as a notch, were considered instead of a single-mode sinusoidal perturbation considered in classical investigations of this instability. Zhang et al. [108] used the fifth- and seventh-order WENO method to simulate the interactions between planar Mach 1.095 and 1.2 shocks with an $\mathrm{SF}_{6}$ gas cylinder in two dimensions in order to qualitatively and quantitatively study the dynamical mechanisms of baroclinic vorticity and circulation generation. The results from the WENO simulations were also compared to the results obtained using the FLASH code. The interface between the vertical cylinder and surrounding air was modeled by a transition layer of finite thickness. In addition to flow visualizations at different evolution times, cylinder lengths, integrated positive and negative vorticity components, normalized circulation, the distributions of the velocity and density gradient, and cylinder aspect ratio as a function of time were extracted from the numerical simulation data. 
A description of the initial conditions for the simulations and of the numerical study is presented in $\S$ 3.1. Density plots from the simulations are compared to experimental PLIF images in $\S 3.2$. The mixing layer width is compared to experimental data and to the predictions of models before and after reshock in $\S 3.3$ and $\S 3.4$, respectively. The time-evolution of the circulation is considered in $\S 3.5$. Comparisons of quantities obtained from simulations using different orders of reconstruction and grid resolutions are presented later in $\S 5$.

\subsection{Numerical simulation parameters}

The initial conditions for the present numerical simulations were taken from the experimental shock tube configuration of Collins and Jacobs [23]. The Mach 1.21 experiment was selected for additional validation of the HOPE code by comparing the numerical results to the highresolution density PLIF images showing the evolution of the instability and to the measured mixing layer amplitude prior to reshock. The experiments were conducted in a shock tube using a novel technique to generate a membrane-less perturbed interface. The entire shock tube had a length of $4.3 \mathrm{~m}$ with a square test section having cross-section $8.9 \mathrm{~cm} \times 8.9 \mathrm{~cm}$ and length $75 \mathrm{~cm}$. The shock was generated through the rupture of a membrane and was launched into a mixture of $75 \%$ air and $25 \%$ acetone by volume [referred to as air(acetone)] at standard room temperature and pressure. The shock then refracted at a perturbed interface separating the air(acetone) mixture and the denser sulfur hexafluoride $\left(\mathrm{SF}_{6}\right)$ gas, giving rise to the Richtmyer-Meshkov instability.

The membrane-less interface was generated through a technique described by Jones and Jacobs [48]. The shock tube contained horizontal slots on two opposite walls. The gases entered the shock tube from opposite ends, flowed toward each other, and exited through the two slots, resulting in a fine, diffuse interface. A perturbation was then generated by gently oscillating the vertical shock tube at a prescribed frequency to establish a standing wave. The diffused interface was estimated to be $0.5 \mathrm{~cm}$ wide, and the oscillations produced a sinusoidal perturbation. Planar laser-induced fluorescence (PLIF) was used to visualize the instability evolution using a mixture of fluorescent acetone with air. The PLIF images were corrected for the non-uniform laser illumination and Beer's law attenuation.

A set of experiments was conducted for shocks with $M a=1.11 \pm 0.01$ and $M a=1.21 \pm 0.02$, and images were captured up to $11 \mathrm{~ms}$ following the initial shock-interface interaction. The pre-shock Atwood number was $A^{-}=0.604$. The evolution of the instability with spikes of heavier fluid penetrating the lighter fluid and bubbles of lighter fluid "rising" in the heavier fluid was investigated. The reshock phase observed when the transmitted shock reflects from the end wall of the test section and interacts with the evolving interface was also described. Concurrent with the arrival of the reflected shock, a reflected rarefaction wave also interacted with the interface as seen in Fig. 4 of [23]. This initial rarefaction wave was created by the rupture of the membrane used to generate the initial shock, was subsequently reflected from the end (top) wall of the shock tube, and then interacted with the evolving interface. This interaction with the reflected rarefaction wave induced the formation of additional complex structure on the evolving interface.

\subsubsection{Initial gas composition}

The experiment was performed using a mixture of air and acetone initially separated from sulfur hexafluoride $\left(\mathrm{SF}_{6}\right)$ by a diffuse interface. Here it is assumed that the gas composition consists of a mixture of $25 \%$ acetone and $75 \%$ air by volume. See Table 1 in Part 1 of this 
report [57] for a summary of the thermodynamic properties of the air(acetone) and $\mathrm{SF}_{6}$ gases. A mixture of $50 \%$ air(acetone) and $50 \% \mathrm{SF}_{6}$ by volume was assumed, yielding a single value of the adiabatic exponent $\gamma=1.24815$. Only the Mach 1.21 experiment is considered in the present report. The conditions in region 1 ahead of the shock are $p_{1}=0.925551$ bar, $T_{1}=296$ $\mathrm{K}$, and $u_{1}=0 \mathrm{~cm} / \mathrm{s}$. Cook, Cabot and Greenough [24] also considered this experiment prior to reshock, and modified their simulation parameters to account for non-ideal effects in the shock tube, such as an effectively smaller Mach number due to the outflow of gas through the slots. In the present investigation, no such modifications are used.

\subsubsection{Computational domain and interfacial perturbation}

To match the shock tube test section dimensions [23], the computational domain has spanwise dimension $L_{y}=8.9 \mathrm{~cm}$, with the perturbed initial interface located $3 \mathrm{~cm}$ from the edge of the shock tube. To obtain a test section length of $75 \mathrm{~cm}$, the total length of the computational domain along the streamwise direction is $L_{x}=78 \mathrm{~cm}$. The adaptive domain capability in the HOPE code [57] allows the initial domain in $x$ to be much smaller than $L_{x}$. In the present simulation, the initial value of $L_{x}$ is chosen to be approximately $9.3 \mathrm{~cm}$ (see Table 2 in $\S 5$ for the values used in the simulations). The computational domain in the $x$ direction is elongated in increments of $3 \mathrm{~cm}$ until a total length of $78 \mathrm{~cm}$ is attained.

As in the experiment, in the numerical simulation the sinusoidal interfacial perturbation (3) had amplitude $a_{0}=0.2 \mathrm{~cm}$ and wavelength $\lambda=5.93333 \mathrm{~cm}$, and the diffuse interface had width $\delta=0.5 \mathrm{~cm}$. Thus, $k a_{0}=0.21 \ll 1$, so that the initial growth is in the linear regime.

\subsection{Qualitative comparison of instability evolution to experimental PLIF images}

In Fig. 6, corrected PLIF density images from the Collins and Jacobs Mach 1.21 shock tube experiment are compared to density plots from the numerical simulations at selected times before reshock: the experimental images are presented in the middle row, the images from the ninth-order WENO simulation are presented in the top row, and the images from the fifth-order WENO simulation are presented in the bottom row. The grid resolution of both simulations was 256 points per initial perturbation wavelength. Very good agreement is observed between the numerical and experimental images. The density from the ninth-order simulation shows sharper roll-ups than that from the fifth-order simulation.

Figure 7 continues this comparison for selected times following reshock. The ninth-order simulation captures the secondary instability within the roll-ups at $t=6 \mathrm{~ms}$. The agreement continues to be very good, although an increasing time discrepancy between the numerical and experimental results develops, with the simulation images lagging in time behind the PLIF images. This time delay can be explained by the arrival of the initial rarefaction wave, which decelerates the evolving interface, causing a progressively larger delay in the time of reshock. The rarefaction wave also causes the formation of small-scale structures on the interface that are amplified during reshock. Note that at late time $(t=10 \mathrm{~ms})$, the experimental data shows increased mixing and fragmentation of large structures. By contrast, the numerical simulation shows that large structures persist. This is due to the excitation of fluctuations in the third spatial dimension caused by the initial rarefaction wave present in the experiment and subsequently amplified by reshock, which is not modeled in the numerical simulation. 

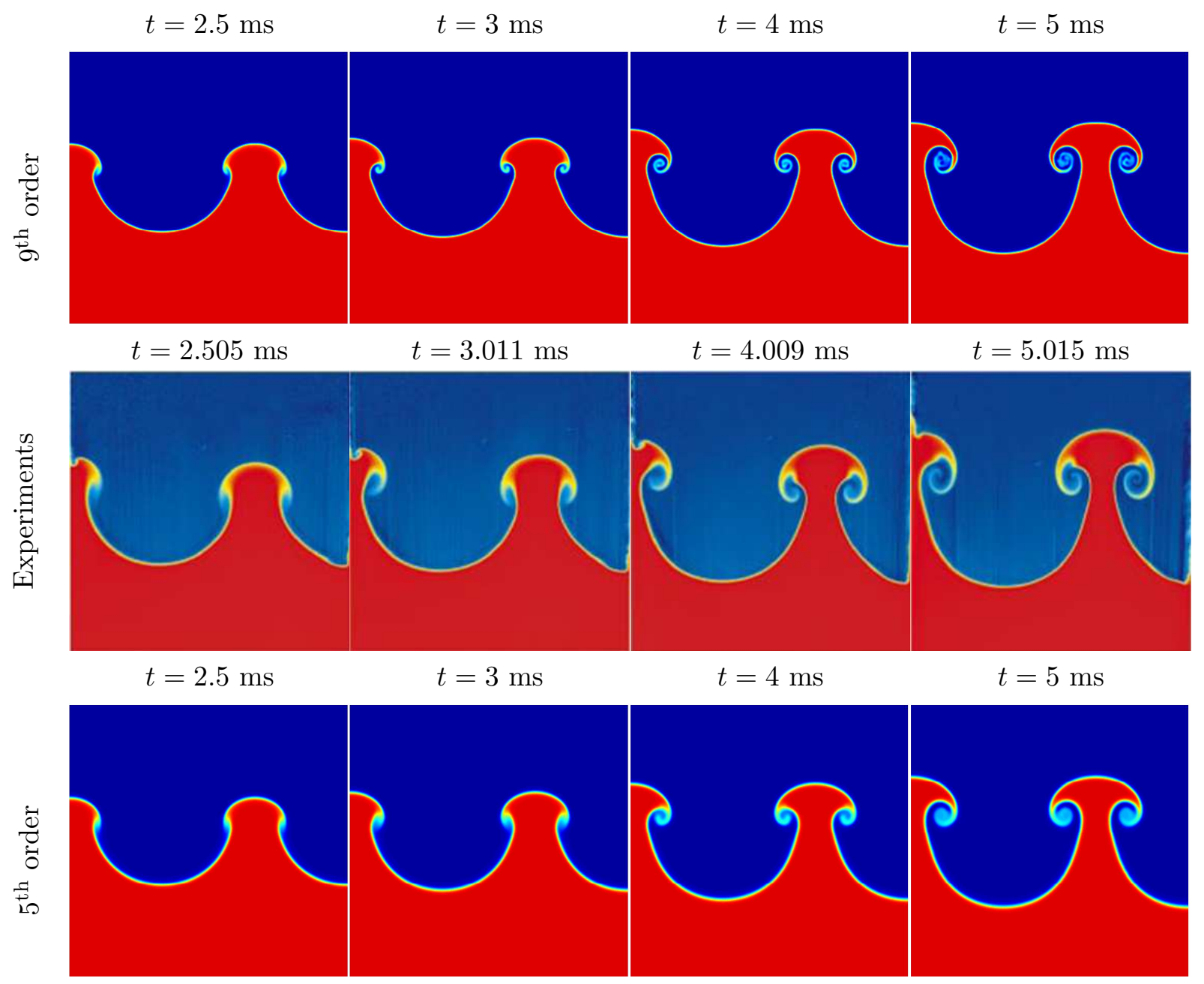

Figure 6: Comparison of corrected PLIF images from the experiment of Collins and Jacobs (middle row) and the density from the ninth-order WENO simulation (top row) and from the fifth-order WENO simulation (bottom row), both on the medium resolution grid. The gases are air(acetone) (blue) and $\mathrm{SF}_{6}$ (red). Note the very good agreement between the experiment and simulation. The ninth-order simulation captures more of the structure of the roll-up observed in the experiment at late times than does the fifth-order simulation. The experimental images are taken from Fig. 6 of Collins and Jacobs [23] (reprinted with the permission of Cambridge University Press). 


$$
t=6 \mathrm{~ms}
$$

$t=6.58 \mathrm{~ms}$

$t=6.88 \mathrm{~ms}$

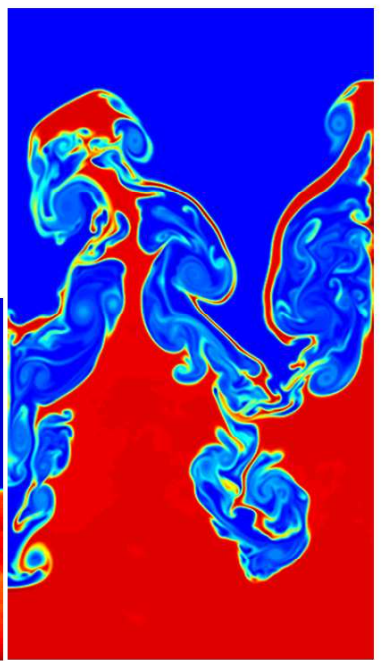

$t=6.006 \mathrm{~ms}$

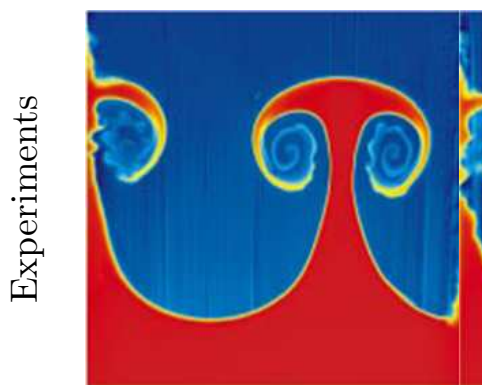

$t=6 \mathrm{~ms}$

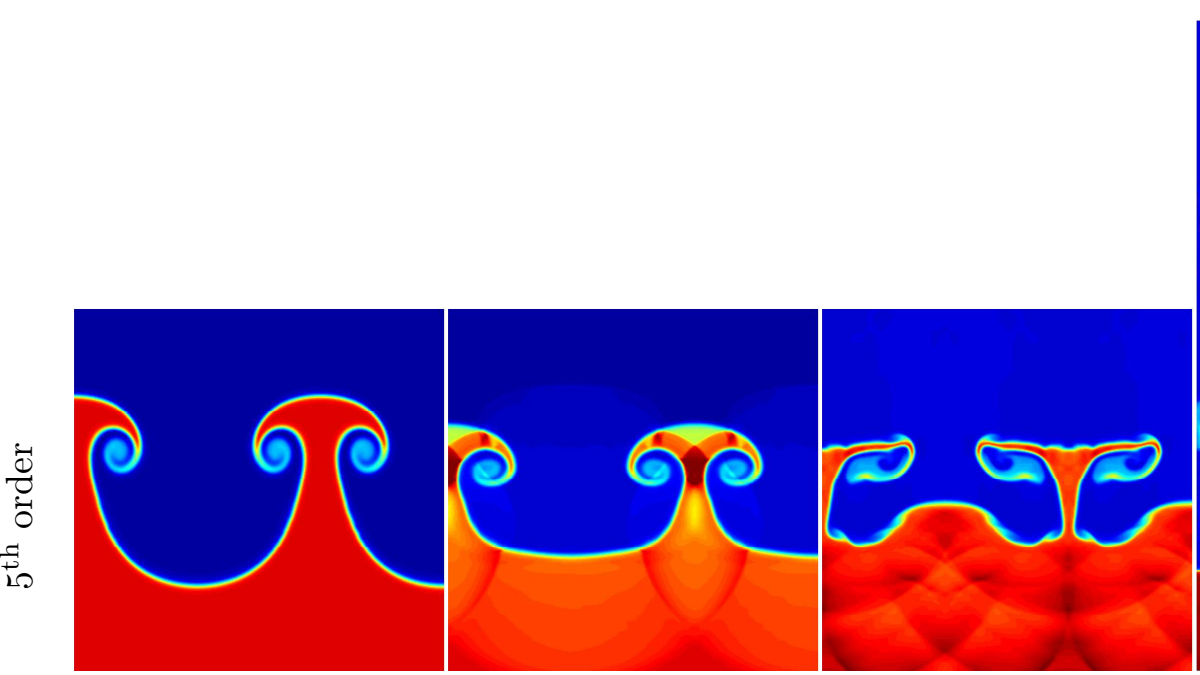

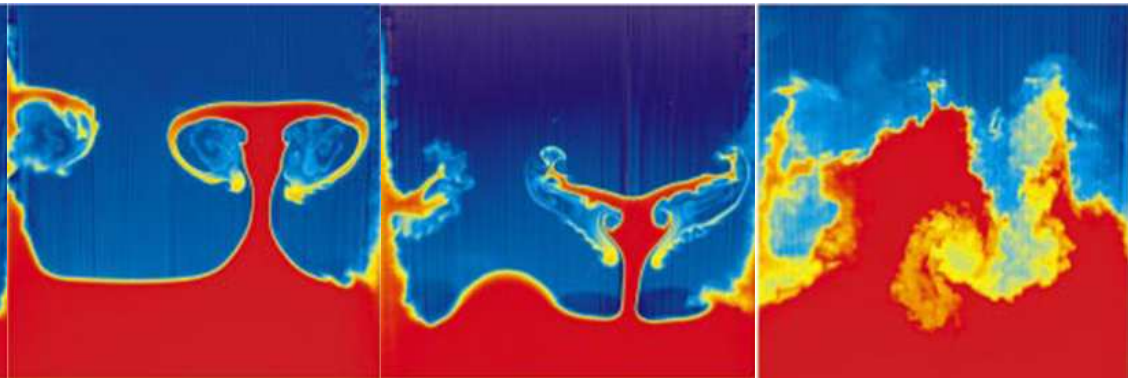

$t=10 \mathrm{~ms}$

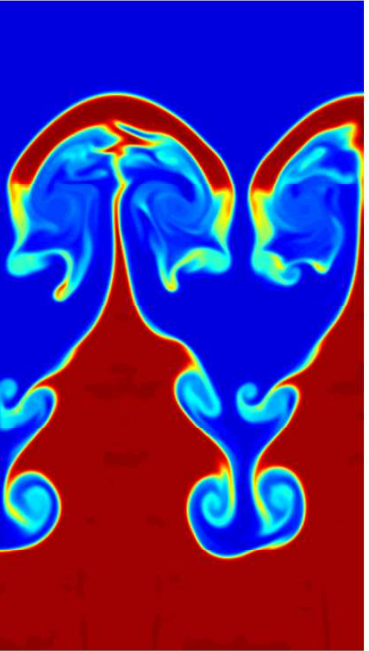

$t=10.02 \mathrm{~ms}$

Figure 7: Further comparison of the corrected PLIF images from the experiment of Collins and Jacobs and the density from the numerical simulation (see Fig. 6). A progressively larger time delay develops between the experimental and simulation images due to the rarefaction wave in the experiments, which is not captured in the simulations. The rarefaction wave decelerates the interface, causing reshock to occur later in time in the experiment compared to the simulation. The experimental images are taken from Fig. 6 of Collins and Jacobs [23] (reprinted with the permission of Cambridge University Press). 


\subsection{Evolution of the amplitude prior to reshock}

In this section, the perturbation amplitude growth from the numerical simulation is compared to the experimentally-measured amplitude from Collins and Jacobs [23] and to the predictions of the models presented in $\S 2$. Figure 8 shows the locations of the interface and shock in the $x-t$ diagram from the simulation. The location of the spike and of the bubble is indicated by the dashed lines. The horizontal distance between the spike and bubble represents the amplitude (see Fig. 4 for a schematic of the total amplitude and the bubble and spike amplitudes). Reshock occurs at $t \approx 6.5 \mathrm{~ms}$ when the shock wave refracts at the evolving interface, generating a transmitted shock in the air(acetone) and a reflected rarefaction wave in the $\mathrm{SF}_{6}$. The reflected rarefaction wave is not plotted in the $x$ - $t$ diagram. Note that the transmitted shock moves faster, as indicated by the change in slope, corresponding to a slow-fast refraction [39]. Following reshock, the interface is compressed (as seen from the kink in the bubble and spike locations) and moves back into the shock tube away from the end wall of the test section. Additionally, the amplitude grows more rapidly than prior to reshock. The increased growth is due to the additional vorticity deposited during reshock on the evolving interface. The reshock (inversion) process occurs over $\approx 0.2-0.3 \mathrm{~ms}$.

\subsubsection{Numerical determination of the mixing layer width}

The mixing layer width is obtained from the mole fraction as follows. In incompressible or variable-density flows, the mole fraction is typically defined as [25]

$$
X(x, y, t)=\frac{\rho(x, y, t)-\rho_{2}}{\rho_{2}-\rho_{1}} .
$$

However, for compressible flows this definition is not desirable, as it is unclear how $\rho_{1}$ and $\rho_{2}$ should be defined. An alternative definition of the mole fraction based on the molecular weights and on the mass fraction is therefore needed. In the present numerical simulations, the mass fraction

$$
m_{2}=\frac{c_{2} M_{2}}{c_{1} M_{1}+c_{2} M_{2}}
$$

of fluid $2\left(\mathrm{SF}_{6}\right)$ is evolved, where $M_{r}$ and $c_{r}$ are the molecular weight and molar concentration of fluid $i$, respectively. By definition, the mass fraction of fluid 1 is

$$
\begin{aligned}
m_{1} & =1-m_{2} \\
& =\frac{c_{1} M_{1}}{c_{1} M_{1}+c_{2} M_{2}} .
\end{aligned}
$$

Then, the mole fraction of fluid 2 can be defined in terms of the molar concentrations by

$$
X=\frac{c_{2}}{c_{1}+c_{2}} .
$$

Using the above definitions, define a mean molecular weight

$$
\bar{M}=\frac{c_{1} M_{1}+c_{2} M_{2}}{c_{1}+c_{2}},
$$

which can also be obtained from the mass fractions $m_{r}$ as

$$
\bar{M}=\frac{M_{1} M_{2}}{M_{1} m_{2}+M_{2} m_{1}} .
$$


Finally, the mole fraction is obtained from the mass fraction $m_{2}$ and $\bar{M}$ as

$$
X=\frac{\bar{M} m_{2}}{M_{2}} .
$$

Equations (156) and (157) yield the desired relation $X=X\left(M_{1}, M_{2}, m_{2}\right)$ independent of the densities, and therefore suitable for compressible flows.

The mixing layer width is obtained by spatially-averaging the mole fraction in the periodic $y$-direction

$$
\langle X\rangle=\frac{1}{L_{y}} \int_{0}^{L_{y}} X(x, y, t) \mathrm{d} y,
$$

where $L_{y}$ is the width of the shock tube in the spanwise direction. The spike and bubble locations, $\ell_{s}(t)$ and $\ell_{b}(t)$, are defined as the $x$ position where $\langle X\rangle \geq \epsilon$ and $\langle X\rangle \leq 1-\epsilon$, respectively, with $\epsilon=0.01$ in the present investigation (corresponding to a $1-99 \%$ criterion in the mole fraction). Therefore, the total mixing layer width is numerically determined by

$$
h(t)=\ell_{b}(t)-\ell_{s}(t) .
$$

Note that this definition of the mixing layer width is quite sensitive to the choice of $\epsilon$. This sensitivity will be discussed elsewhere [59].

When comparing the numerical simulation data to the predictions of the models presented in $\S 2$, the following conventions are used. The wavenumber is adjusted to account for the diffuse interface by including the growth reduction factor $\psi$ (see $\S 2.1 .5$ ) by

$$
k \longrightarrow \frac{k}{\psi}
$$

Furthermore, the time and initial velocity are rescaled according to

$$
\tau=k v_{0} t, v_{0}=k A^{+}[u] a_{0}^{+},
$$

where $a_{0}^{+}$is the post-shock amplitude and $A^{+}$is the post-shock Atwood number. Note that the post-shock amplitude $a_{0}^{+}$is determined by multiplying the pre-shock amplitude $a_{0}^{-}$by the compression factor

$$
\eta_{\text {comp }}=1-\frac{[u]}{u_{\text {shock }}},
$$

where $u_{\text {shock }}$ is the velocity of the shock. The values of the pre-shock and post-shock quantities are summarized in Table 1.

Note that all of the models predict the growth rate of the mixing layer $\mathrm{d} a / \mathrm{d} t$, rather than $a$. Therefore, in the following sections, the mixing layer amplitude $a$ is numerically computed by solving an initial value problem (here using a fourth-order Runge-Kutta scheme [16]).

\subsubsection{Quantitative comparison to experimental data and simulated Schlieren im- ages}

In Fig. 9, the mixing layer amplitude from the simulation (blue) is compared with the experimental measurements from Collins and Jacobs [23] (red). The mixing layer begins growing immediately after the passage of the initial shock. The initial rapid growth saturates at approximately $4 \mathrm{~ms}$, and then reshock occurs approximately $2.5 \mathrm{~ms}$ later. During reshock, the 


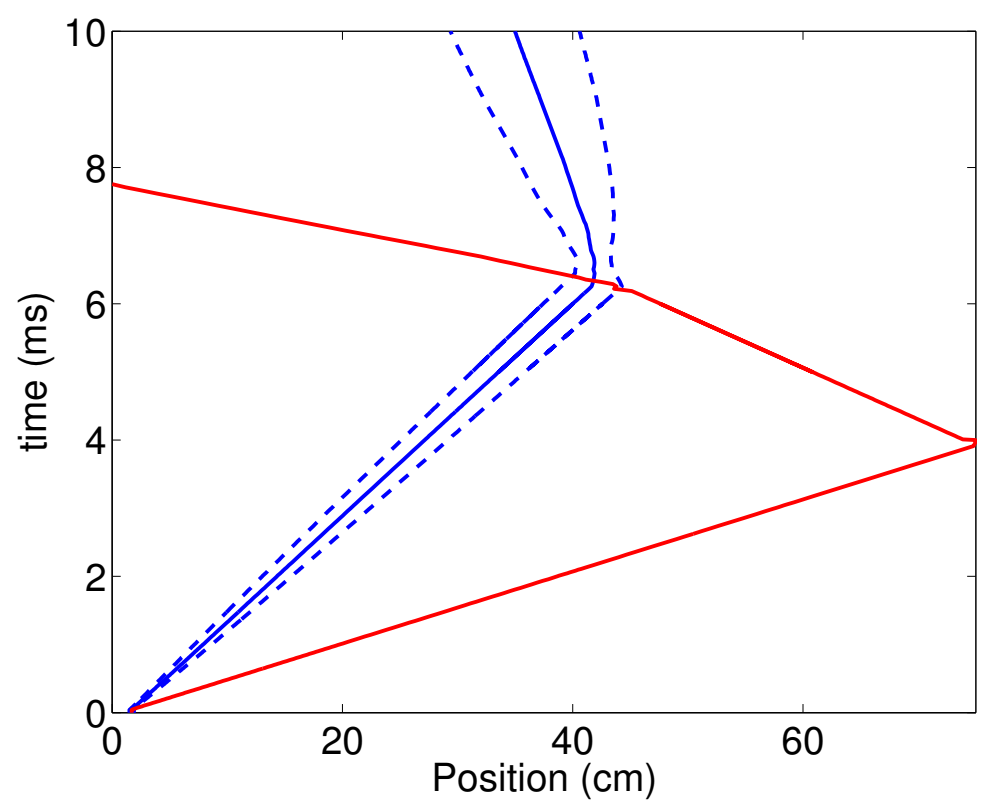

Figure 8: The $x$-t diagram showing the position of the interface (solid blue line), shock (red line), and bubble and spike locations (dashed blue lines) from the simulation. The horizontal distance between the spike and bubble represents the total amplitude.

\begin{tabular}{|c||c|c|}
\hline & pre-shock & post-shock \\
\hline$a_{0}(\mathrm{~cm})$ & 0.2 & 0.1614 \\
$A$ & 0.605 & 0.489 \\
{$[u](\mathrm{cm} / \mathrm{s})$} & & 6902 \\
$u_{\text {shock }}(\mathrm{cm} / \mathrm{s})$ & 35775 & 18085 \\
$\eta_{\text {comp }}$ & & 0.8071 \\
\hline
\end{tabular}

Table 1: Pre- and post-shock values obtained from the simulation. 


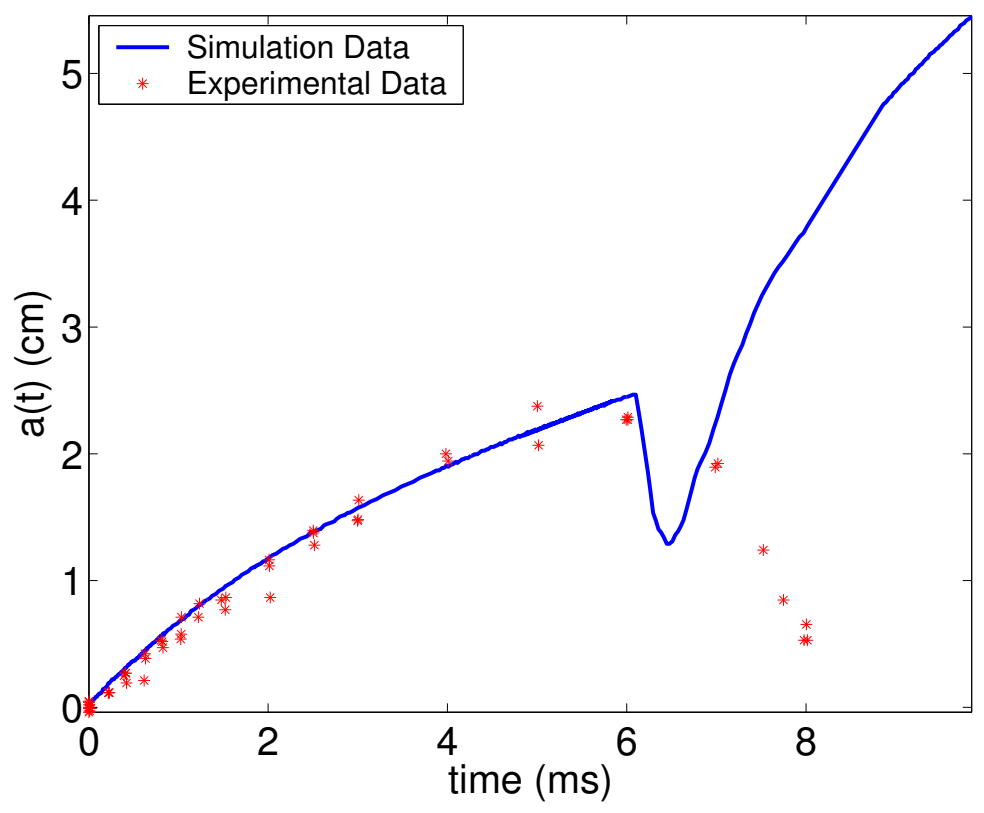

Figure 9: Comparison of the mixing layer width obtained from the numerical simulation to the experimental data from Collins and Jacobs in the case of a Mach 1.21 incident shock. Note the excellent agreement up to reshock. The subsequent large discrepancy is due to the arrival of the initial rarefaction wave not modeled in the simulation.

interface is compressed by approximately $1 \mathrm{~cm}$ and then grows rapidly. Comparison of the numerical data with the experimental data points shows excellent agreement up to reshock. The difference observed after reshock is due to the arrival of the initial rarefaction wave that is not modeled in the present simulations, as discussed in $\S 3.1$. The initial rarefaction wave decelerates the interface and, thus, prolongs the reshock phase to after $8 \mathrm{~ms}$ in the experiments. The large difference observed after reshock emphasizes the importance of the reflected rarefaction wave on the evolution of the instability. The instability evolution in this experiment is essentially two-dimensional prior to reshock (by virtue of the manner in which the initial perturbation was produced), and becomes three-dimensional following reshock, as the shockinterface interaction excites fluctuations in all spatial directions. Thus, it may be expected that a two-dimensional numerical simulation can reproduce the experimentally-measured amplitude growth prior to reshock. However, three-dimensional effects presumably become important following reshock, and three-dimensional numerical simulations are necessary to correctly capture the mixing layer width evolution and other quantities. According to Collins and Jacobs [23], the best fit to their amplitude growth data is given by the Sadot et al. [80] model.

The wave structure observed in the numerical simulation can be investigated using simulated density Schlieren images. The definition of the Schlieren function $\Phi$ used here is [66]

$$
\Phi=\exp \left[-\alpha\left(m_{2}\right) \frac{|\nabla \rho|}{\max |\rho|}\right]
$$

where $m_{2}$ denotes the mass fraction of $\mathrm{SF}_{6}$, and

$$
\alpha\left(m_{2}\right)=\left\{\begin{array}{ll}
20 & \text { if } m_{2}>\bar{\rho} \\
100 & \text { if } m_{2}<\bar{\rho}
\end{array},\right.
$$


where $\bar{\rho}=\left(\rho_{1}+\rho_{2}\right) / 2$. The Schlieren shows the density gradients associated with waves and fine-scale mixing structures.

Figure 10 shows a time sequence of simulated density Schlieren images from the numerical simulation during reshock and at late times. The image sharply captures the diffuse interface, the complex wave structure during reshock, and the small-scale mixing structures at late times. Note the focusing effect of the waves and the inversion of the bubble transforming into the spike and vice versa. The images indicate that reshock occurs over a timescale of approximately $0.2-$ $0.3 \mathrm{~ms}$. The image corresponding to $t=11 \mathrm{~ms}$ also shows the arrival of the reflected rarefaction after it has reflected from the end wall of the test section. The reflected rarefaction wave was produced by the reshock.

\subsubsection{Comparison to the predictions of impulsive models}

In this section, numerical and experimental data are compared with the predictions of the linear instability models described in $\S 2.1$. In Fig. 11, the mixing layer amplitude from the simulation is compared with the Richtmyer [87] model [see Eq. (11)], the Meyer and Blewett [70] model [see Eq. (15)], the Fraley [34] model [see Eq. (17)] and the Vandenboomgaerde et. al. [99] model [see Eq. (23)]. These models capture the initial linear growth rate of the mixing layer for normalized times $\tau \leq 1$. For times $\tau>1$, nonlinear effects become significant and the models significantly overestimate the mixing layer amplitude. For the initial conditions considered here, the Richtmyer model gives the smallest slope as it uses the postshock Atwood number and amplitudes. The Meyer-Blewett model uses the post-shock Atwood number, but averages the pre- and post-shock amplitudes, resulting in the largest slope. The Vandenboomgaerde model averages the pre- and post-shock amplitudes by the pre- and postshock Atwood numbers, respectively, and thus, has a slightly smaller slope than that predicted by the Meyer-Blewett model, but larger than the slope predicted by the Richtmyer model. The Fraley model, corresponding to the exact initial slope, has a slope intermediate between those predicted by the Meyer-Blewett and the Vandenboomgaerde models. This indicates that the impulsive models can successfully capture the linear growth of the mixing layer, and it is difficult to determine which model agrees best with the data. However, this is not the case for all initial conditions, as a normalized growth rate analysis [72] would show. In this analysis, the normalized growth rate is plotted as a function of the shock strength. The Fraley solution is taken to be the exact solution and the impulsive models are compared to this solution. For large shock strengths, the impulsive models exhibit large deviations from the Fraley solution, indicating that they are no longer reliable.

Overall, all of the impulsive models correctly capture the initial growth of the mixing layer for $\tau<1$. After the initial linear phase, the models do not accurately capture the late-time evolution of the interface, as nonlinear effects become significant and the amplitude saturates. Consequently, the late-time evolution $(\tau>1)$ is often referred to as the nonlinear growth phase.

\subsubsection{Comparison to the predictions of the Jacobs-Sheeley vortex model}

The numerical data and the experimental data are compared in Fig. 12 with the predictions of the vortex model proposed by Jacobs and Sheeley [47] [see Eq. (40)] in the case $k a_{0}^{+}=0$ and $k a_{0}^{+}=2$. The first value $k a_{0}^{+}=0$ corresponds to modeling the deposition of vorticity on the interface by two large point vortices located at the midpoints of the sinusoidal perturbation. The second value $k a_{0}^{+}=2$ accounts for the delay between the deposition of vorticity and its roll-up into two large vortices of opposite sign. While the model captures an asymptotic $1 / t$ 
$t=6.38 \mathrm{~ms}$

$t=6.48 \mathrm{~ms}$

$t=6.58 \mathrm{~ms}$

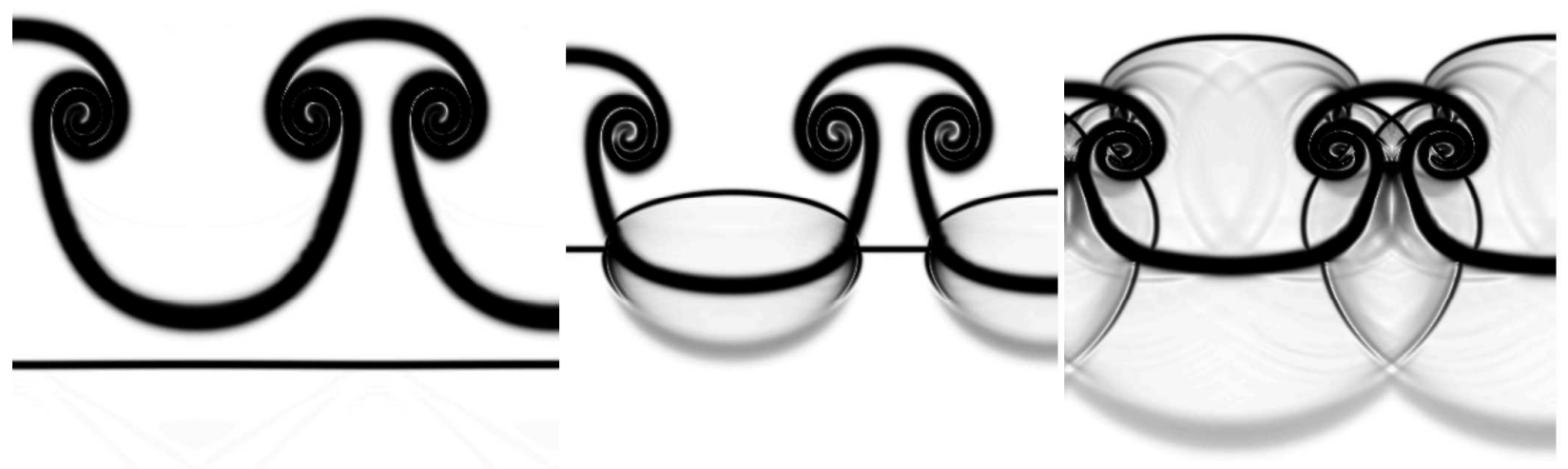

$t=6.68 \mathrm{~ms}$

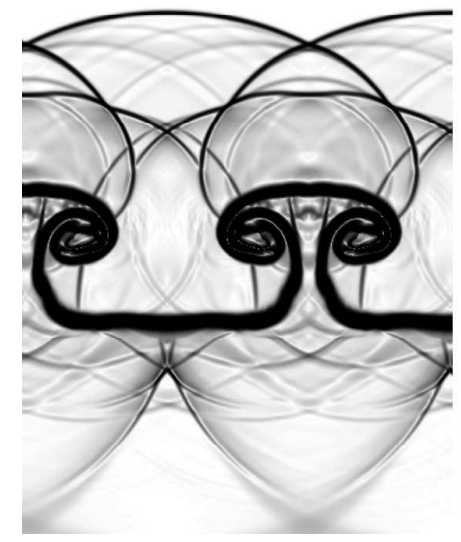

$t=7.18 \mathrm{~ms}$

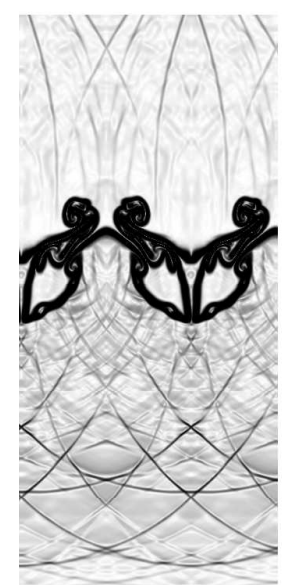

$t=6.78 \mathrm{~ms}$

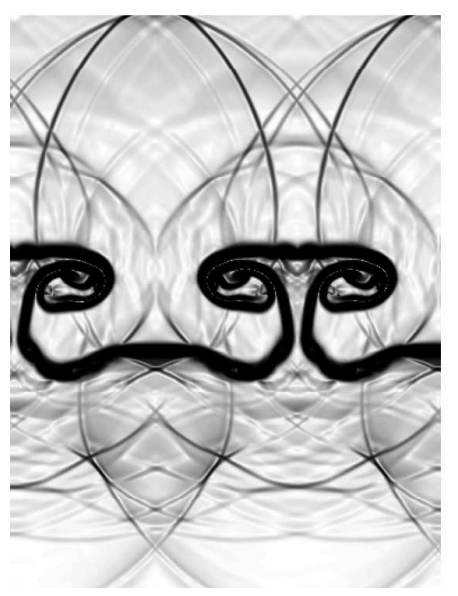

$t=11 \mathrm{~ms}$

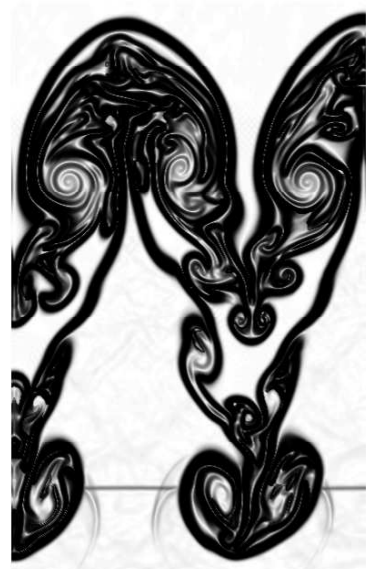

$t=7 \mathrm{~ms}$

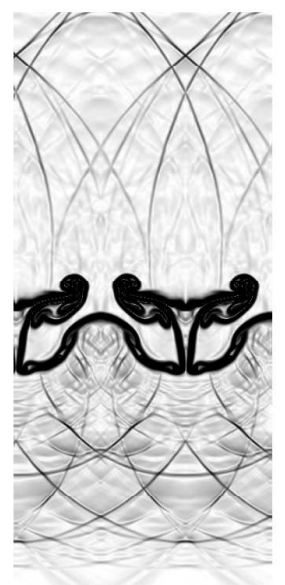

$t=18 \mathrm{~ms}$

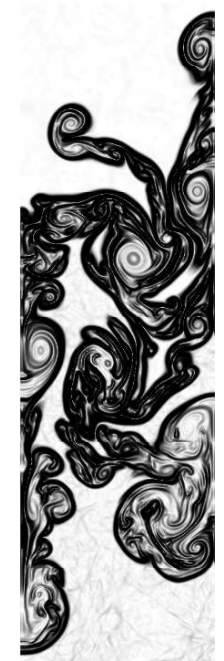

Figure 10: Simulated density Schlieren images of the two-dimensional, single-mode RichtmyerMeshkov instability illustrating the diffuse interface, complex wave structure during reshock, and the small-scale structures at late times. 
amplitude growth, both the numerical and experimental data are in poor agreement with the predictions of the model. The model underestimates the mixing layer width for $k a_{0}^{+}=0$, and overestimates the mixing layer width for $k a_{0}^{+}=2$. This is expected, as the model is based on the assumption that the initial vorticity deposited by the shock immediately coalesces into cores and does not account for the finite-time formation of the cores.

\subsubsection{Comparison to the predictions of perturbation models}

The numerical data and the experimental data are compared with the predictions of the nonlinear models summarized in $\S 2.3$. In Fig. 13, experimental and numerical data are compared with the perturbation series solutions of Zhang and Sohn [107] [see Eq. (54)], and the perturbation series solution of Vandenboomgaerde et al. [98] [see Eq. (63)] of degree 9 and 11. The comparison shows that the perturbation series successfully capture the initial growth but quickly diverge. In particular, note that the result for the eleventh-order Vandenboomgaerde perturbation series has a smaller radius of convergence than the ninth-order series. To expand the radius of convergence, the series are extended via Padé approximants.

In Fig. 14, the experimental and numerical data are compared with the predictions of the nonlinear models extended via Padé approximants. Shown are the $P_{2}^{0}$ Padé approximant of Zhang and Sohn (see Eq. 55) and the $P_{6}^{4}$ Padé approximant of Vandenboomgaerde [see Eq. (66)]. In addition, the empirical model of Sadot et al. [80] [see Eq. (107)] is also presented. Note the excellent agreement between the Padé models and the measured data for both the linear and nonlinear regime. The models show slight variations in their respective predictions. In particular, the Padé approximant of Vandenboomgaerde appears to capture the correct behavior at early times for $\tau<1$, but overestimates the width for later times. The Padé approximant of Zhang and Sohn underestimates the width for $\tau<1$, but gives the correct behavior for later times.

To determine which of the three models gives the amplitude growth in best agreement with the simulation data, the ratio between the Zhang-Sohn (Padé), Vandenboomgaerde (Padé) and Sadot models, and the simulation data, $h_{\text {model }}(t) / h_{\text {sim }}(t)$, are presented in Fig. 15 . The figure indicates that the Zhang-Sohn Padé model is in best agreement with the numerical simulation data. Collins and Jacobs [23] report that their data is in best agreement with the Sadot model.

\subsection{Evolution of the mixing layer amplitude following reshock and compar- ison to reshock models}

The mixing layer amplitude following reshock is investigated here. The reshock in the simulation occurs at nearly the same time as in the experiment, as confirmed by both the $x$ - $t$ diagram and the mixing layer amplitude. The mixing layer width is compared to the predictions of the Mikaelian [71] reshock model and to that of the Brouillette-Sturtevant [14] reshock model in Fig. 16. Note that the flow following reshock is essentially incompressible (see $\S 4.3$ ).

In order to fit the models, the numerical data was shifted in time and the amplitude at reshock was subtracted out so that the new origin coincides with the conditions following reshock. For the Mikaelian model of Eq. (141), the quantities $[u]_{1}=8000 \mathrm{~cm} / \mathrm{s}$ and $A_{1}^{+}=$ 0.6448 from the simulation are used. For the Brouillette-Sturtevant model of Eq. (142), the expression

$$
\left(\frac{\mathrm{d} h}{\mathrm{~d} t}\right)_{1}=2 k\left(\frac{[u] A^{+} a_{0}^{+}}{\psi^{+}}+\frac{[u]_{1} A_{1}^{+} a_{1}^{+}}{\psi_{1}^{+}}\right)
$$




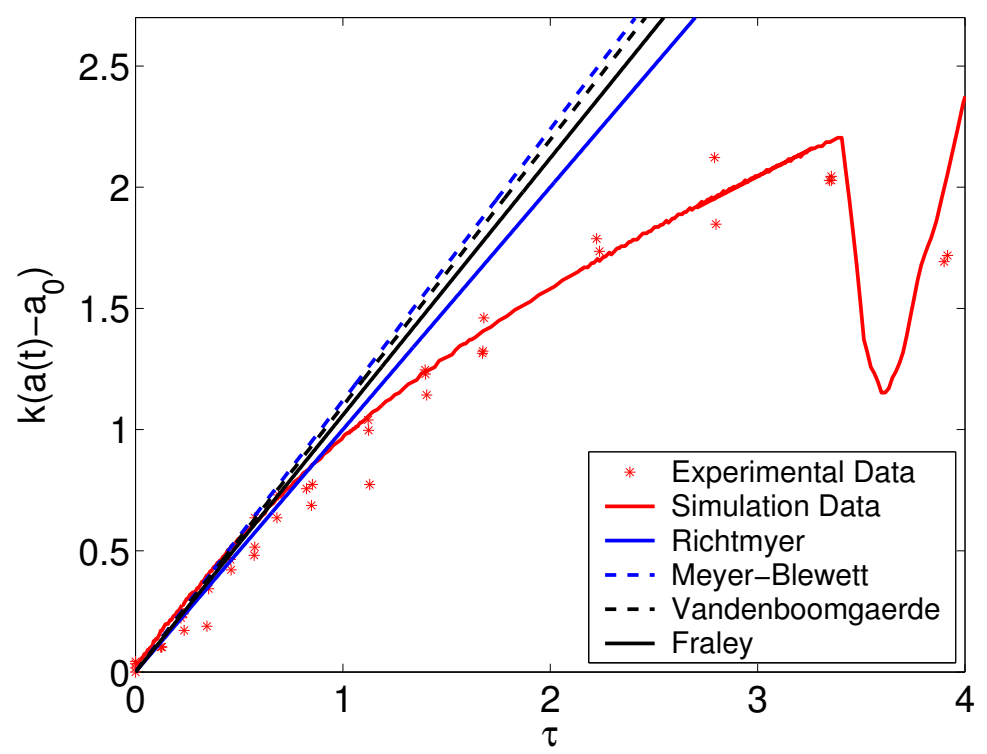

Figure 11: The normalized mixing layer amplitude $k\left[a(t)-a_{0}\right]$ from the numerical simulation and experimental data, together with the predictions from linear models. Time is rescaled such that $\tau=k v_{0} t$, where $v_{0}=k A^{+}[u] a_{0}^{+}$. All linear instability models give good predictions for the initial mixing layer growth. For $\tau>1$, nonlinear effects become relevant and linear models overestimate the mixing layer amplitude.

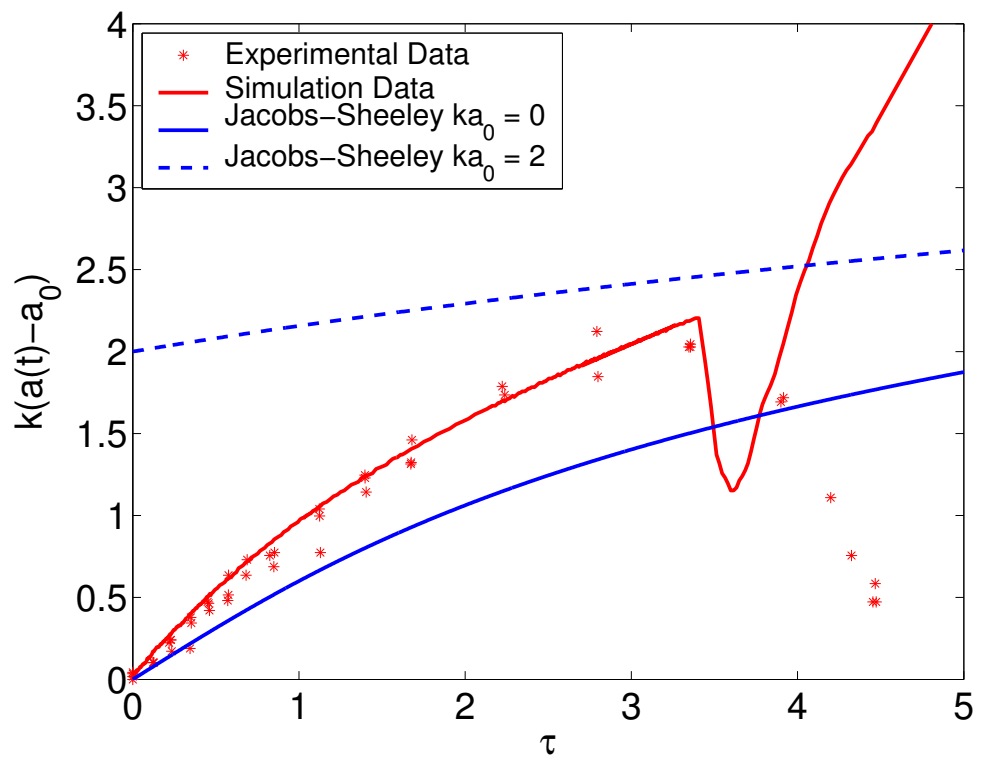

Figure 12: The normalized mixing layer amplitude $k\left[a(t)-a_{0}\right]$ from the numerical simulation and experimental data, together with the predictions from the Jacobs-Sheeley vortex model. When $k a_{0}=0$ the model underestimates the mixing layer width, and when $k a_{0}=2$ the model overestimates the mixing layer amplitude. 

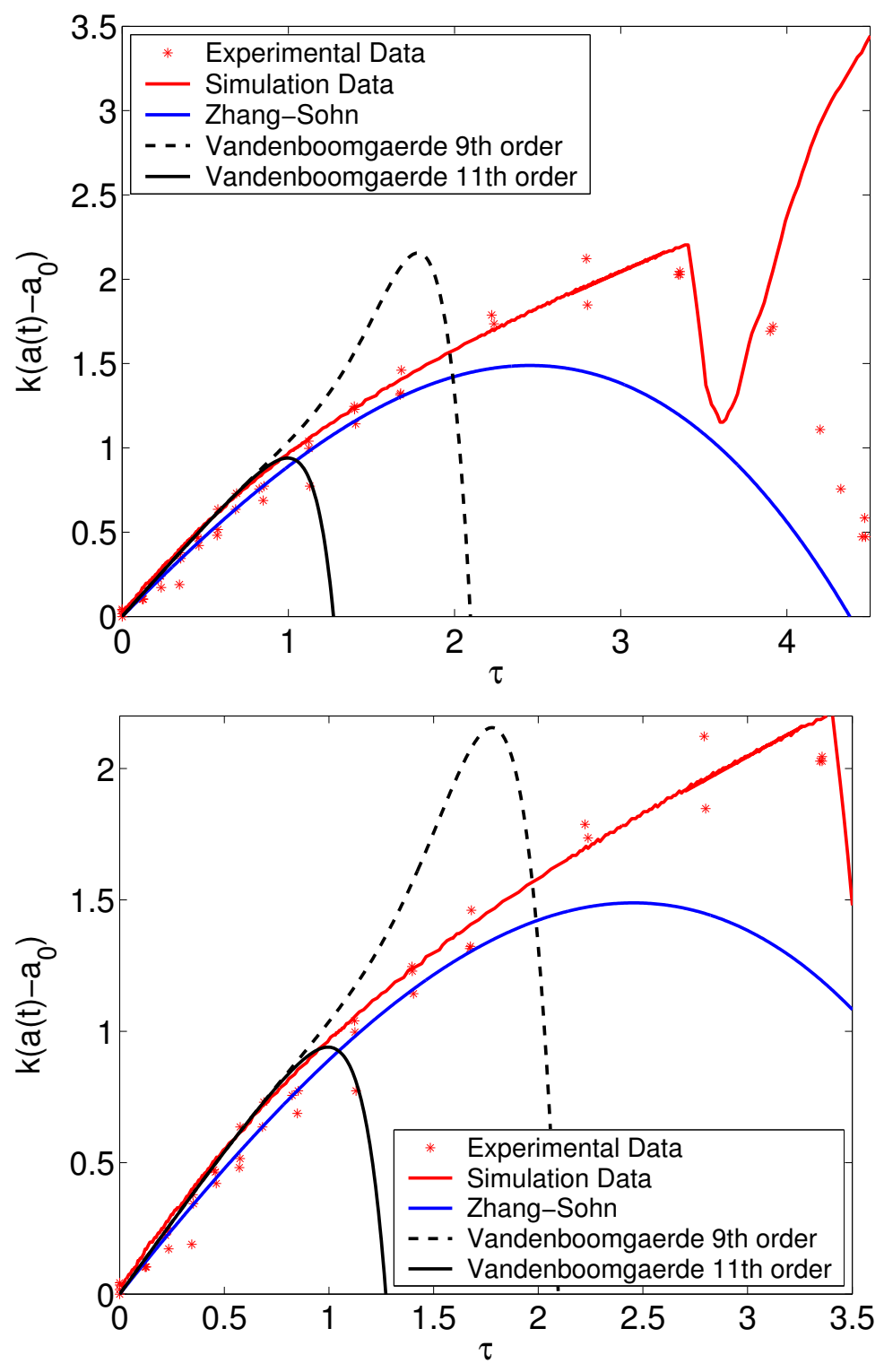

Figure 13: The normalized mixing layer amplitude $k\left[a(t)-a_{0}\right]$ from the numerical simulation and experimental data, together with the predictions from the Padé models of Zhang and Sohn and Vandenboomgaerde. The series captures the initial growth of the mixing layer into the nonlinear regime, but diverges rapidly. The Vandenboomgaerde eleventh-order series diverges sooner than the ninth-order series. 

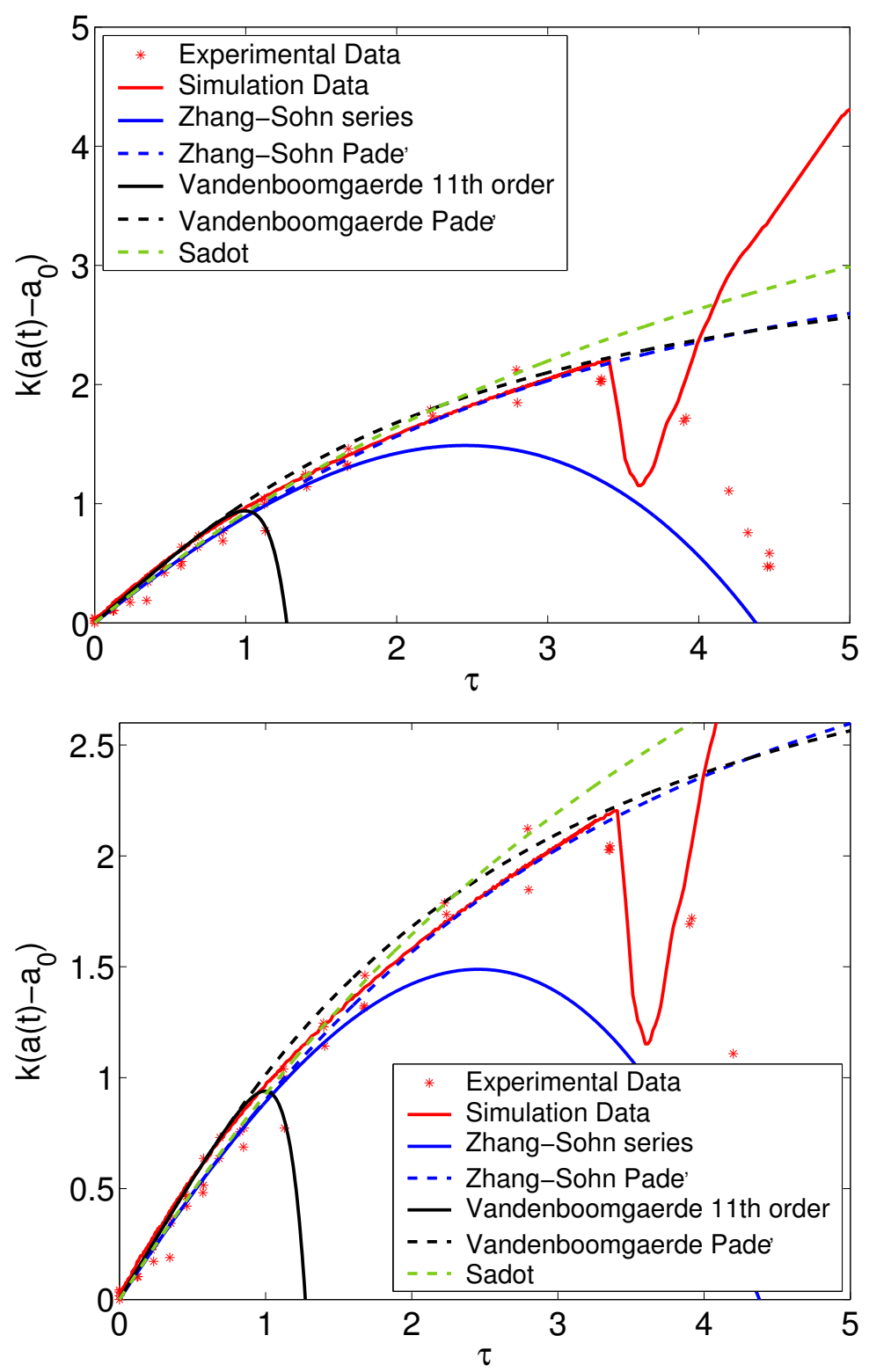

Figure 14: The normalized mixing layer amplitude $k\left[a(t)-a_{0}\right]$ from the numerical simulation and experimental data, together with the predictions of the perturbation series models of Zhang and Sohn and Vandenboomgaerde extended via Padé approximants. The prediction of the empirical model of Sadot is also shown. Note the excellent agreement between the models and the measured amplitude. 


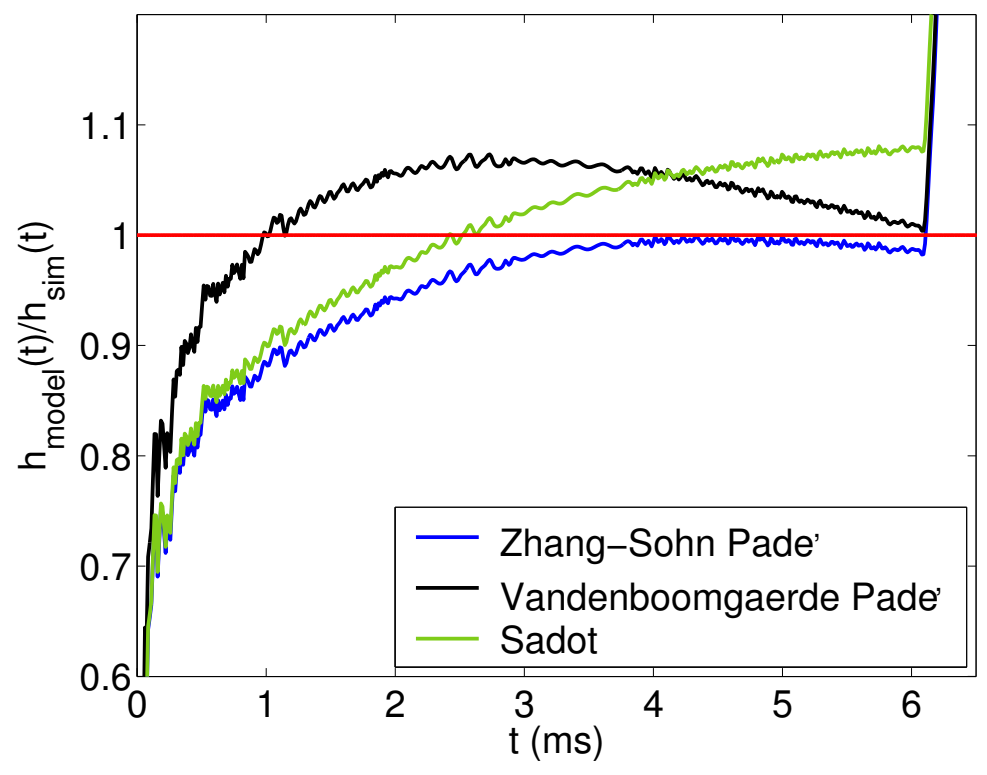

Figure 15: The predictions of the Zhang-Sohn (Padé), Vandenboomgaerde (Padé), and Sadot models for the amplitude divided by the numerical simulation data, $h_{\text {model }}(t) / h_{\text {sim }}(t)$. Well into the nonlinear regime, and just before reshock, the prediction of the Zhang-Sohn model is closest to that of the simulation.

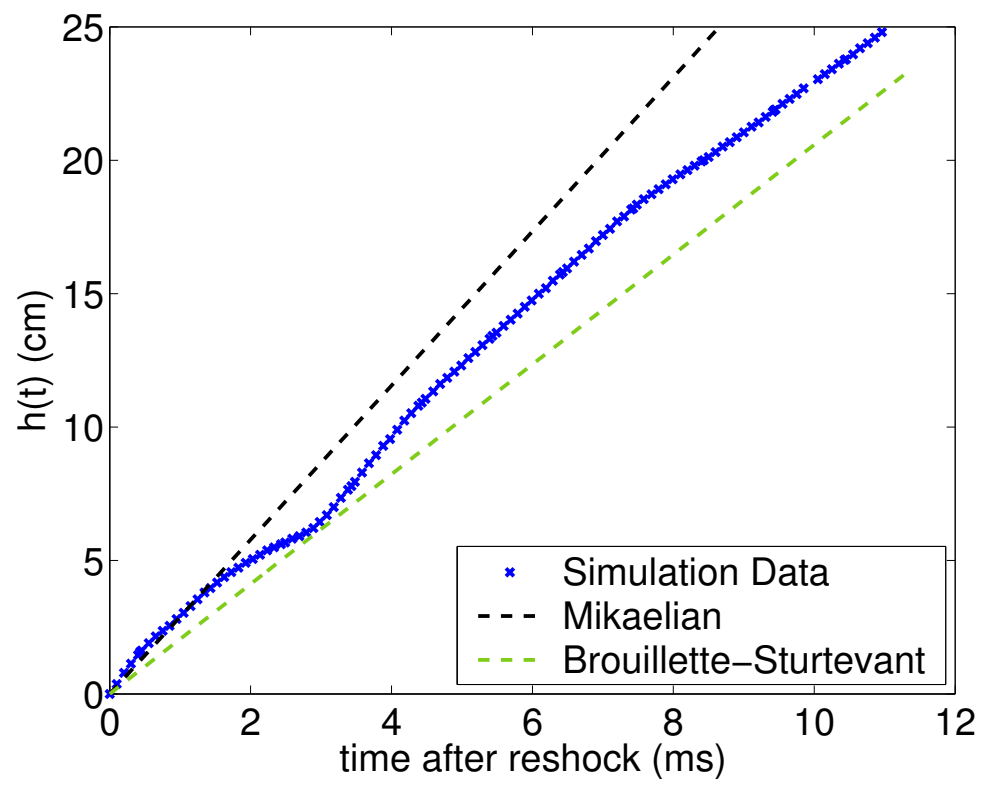

Figure 16: The mixing layer width $h(t)$ of a reshocked interface and the predictions of the Mikaelian and Brouillette-Sturtevant models. The simulation data was shifted in time and the amplitude at reshock was subtracted out so that the new origin coincides with the conditions at reshock. 
was used, where $a_{1}^{+}=1.5 \mathrm{~cm},[u]_{1}=8000 \mathrm{~cm} / \mathrm{s}$, and the value of $\psi_{1}^{+}$is extrapolated as follows. First recall that $\psi$ is a linear function in $\bar{\delta} / \lambda$ [see Eq. (30)], where $\bar{\delta}$ is the diffusive mixing layer width linear in the post-shock perturbation amplitude $a_{0}^{+}$. As $a_{1}^{+} \approx 10 a_{0}^{+}$and the value of $\psi^{+}$is known, the constant $C$ in Eq. (30) can be determined as a function of $\bar{\delta} / \lambda$. Then, substituting the new value for $a_{1}^{+}$gives the new value of $\psi_{1}^{+}$.

Note that the simulation data agrees very well with the prediction of the Mikaelian model at early times $(t>2 \mathrm{~ms})$ following reshock. Vetter and Sturtevant [100] also concluded that their experimental results were in excellent agreement with the prediction of the Mikaelian model. For late times, the Mikaelian model tends to overestimate the growth of the mixing layer. The Brouillette-Sturtevant model tends to underpredict the simulation data at all times following reshock. Using the values above yields $(\mathrm{d} h / \mathrm{d} t)_{1}=1444.35 \mathrm{~cm} / \mathrm{s}$ and $(\mathrm{d} h / \mathrm{d} t)_{1}=1029.05 \mathrm{~cm} / \mathrm{s}$ for the Mikaelian and Brouillette-Sturtevant models, respectively.

\subsection{Circulation deposition on the interface}

The circulation deposited on the interface is computed here. The circulation $\Gamma$ is defined as the line integral of the velocity field $\mathbf{u}$ around a closed contour $C$

$$
\Gamma(t)=\oint_{C} \mathbf{u} \cdot \mathrm{d} \mathbf{r},
$$

where $\mathrm{d} \mathbf{r}$ is the unit tangent to the oriented contour $C$. Using a well-known vector identity, the above expression can be alternatively written in terms of the vorticity field $\omega$ as

$$
\begin{aligned}
\Gamma(t) & =\int_{S}(\nabla \times \mathbf{u}) \cdot \mathrm{d} \mathbf{S} \\
& =\int_{S} \omega \cdot \mathrm{d} \mathbf{S},
\end{aligned}
$$

where $S$ is the surface enclosed by $C$ and $\mathrm{d} \mathbf{S}$ is the outward oriented area element.

For two-dimensional simulations, the vorticity field and the outward oriented area element are parallel. Therefore, the circulation can be computed on a uniform rectangular grid with spacings $\Delta x$ and $\Delta y$ using [92]

$$
\Gamma(t)=\sum_{i, j} \omega\left(x_{i}, y_{j}, t\right) \Delta x \Delta y
$$

where the double summation is over all of the grid points in the domain.

Figure 17 shows the circulation computed to late times. Prior to reshock, the circulation should be a constant in the absence of molecular dissipation. However, numerical dissipation and fluctuations in the density and pressure fields in the present simulations contribute to a very small growth in time following an initial transient. Following reshock, the circulation immediately increases by a factor of $\approx 10$ and increases rapidly following the interaction at $t \approx 11 \mathrm{~ms}$ with the reflected rarefaction wave until $t \approx 15 \mathrm{~ms}$, and then begins to decrease. Wave interactions with the interface inhibit the decay of the circulation for long times following reshock. 


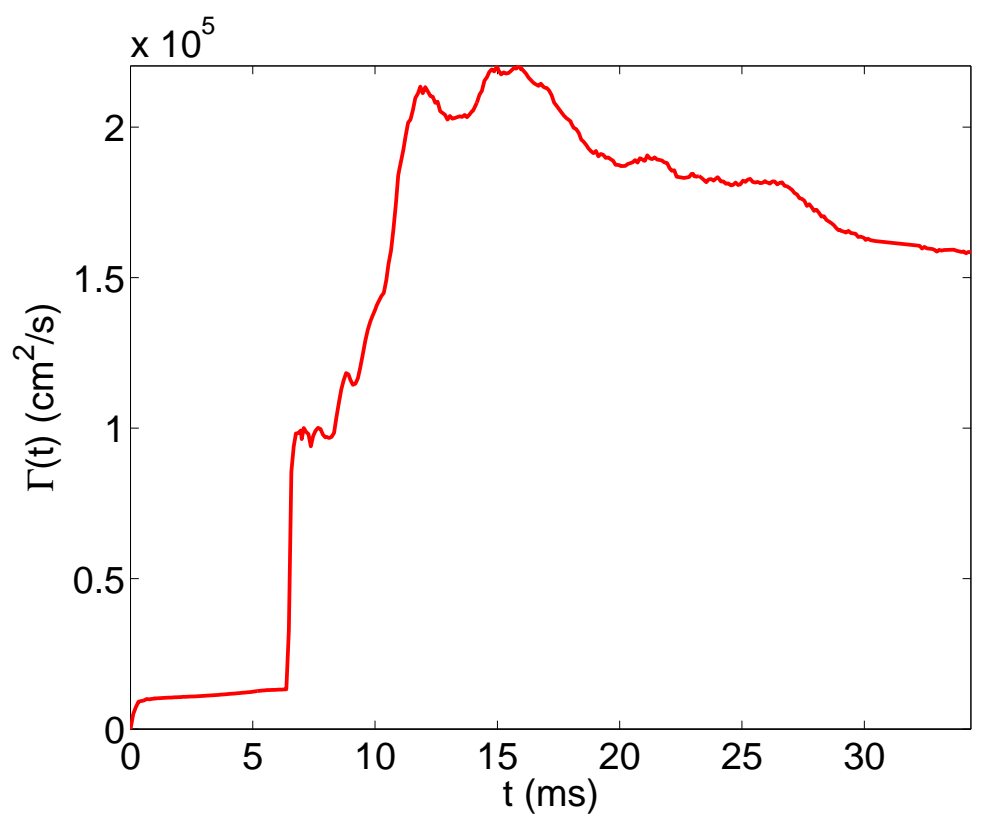

Figure 17: The circulation $\Gamma(t)$ computed to late time. Note the initial slow growth of the circulation until reshock occurs, followed by a rapid increase until $t \approx 15 \mathrm{~ms}$. The circulation decreases thereafter. 


\section{Local and global analysis of mixing in two-dimensional, single- mode Richtmyer-Meshkov instability with reshock}

Presented in this section is a comprehensive investigation of mixing induced by the twodimensional Richtmyer-Meshkov instability with reshock. The analysis of mixing considers both local and global quantities. Local analysis refers to an investigation of a quantity at a fixed time: two types of quantities are considered-mixing fractions and spectra. Mixing fractions are quantities averaged across the statistically-homogeneous (periodic) $y$-direction (so that they are only a function of the streamwise coordinate $x$ and time) characterizing the extent and efficiency of mixing. Spectra of quantities inside the mixing layer are obtained as a function of the one-dimensional wavenumber $k$ by Fourier-transforming along the periodic (spanwise) direction. Global analysis refers to an investigation of a wavenumber-integrated spectrum (a statistic) or a volume-integrated quantity (a mixing fraction or primitive field) as a function of time. Very little previous investigation of these quantities in Richtmyer-Meshkov instability-induced mixing has been performed [103, 75]. However, Youngs [102, 103] and Cook and Dimotakis [25] have conducted an investigation of the evolution of mixing fractions and energy spectra for Rayleigh-Taylor instability-induced mixing.

The analysis is applied to the simulation of the Richtmyer-Meshkov instability initialized using the Mach 1.21 Collins and Jacobs shock tube experiment, and using fifth-order WENO reconstruction and a grid resolution of 256 points per initial perturbation wavelength, as discussed in $\S 3$. To investigate the late-time decay of the mixing following reshock, the boundary condition at the end of the simulated test section is modified to outflow so that reflected waves following reshock exit the computational domain and no longer interact with the evolving mixing layer (the decay regime, as distinct from the quasi-decay regime, in which reflected waves are permitted to interact with the mixing layer). Particular emphasis is also placed on comparing mixing and spectral quantities before and after reshock. The objective of this comprehensive investigation is to quantitatively characterize the mixing induced by the single-mode Richtmyer-Meshkov instability before and after reshock in two dimensions, and to appreciate the manifestations of the reflected waves in the evolution of the reshocked interface.

Note that in the present investigation, the initial condition is deterministic. Furthermore, only one realization of the flow is simulated. Thus, the profiles, spectra, and statistics computed in subsequent sections exhibit variations that would otherwise be reduced if an ensemble average of realizations with a stochastic initial condition were used.

\subsection{Time-evolution of mole, volume fraction, and mixing fraction profiles}

Several averages must be introduced to define spanwise-averaged quantities across the mixing layer (streamwise profiles), as well as fluctuations required to define energy spectra. Let angle brackets denote an instantaneous average of a function $\phi(x, y, t)$ over the periodic (spanwise) direction $y$ with length $L_{y}$ :

$$
\langle\phi\rangle(x, t)=\frac{1}{L_{y}} \int_{0}^{L_{y}} \phi(x, y, t) \mathrm{d} y,
$$

which can be interpreted as an instantaneous Reynolds average in the present investigation (see [103] for the three-dimensional analog of this average). The Reynolds fluctuating field is

$$
\phi(x, y, t)^{\prime}=\phi(x, y, t)-\langle\phi\rangle(x, t) .
$$


Then, the instantaneous Favre average of $\phi$ is

$$
\widetilde{\phi}(x, t)=\frac{\langle\rho \phi\rangle}{\langle\rho\rangle},
$$

which is used for the statistical analysis of variable-density and compressible flows. The Favre fluctuating field is

$$
\phi(x, y, t)^{\prime \prime}=\phi(x, y, t)-\widetilde{\phi}(x, t) .
$$

Thus, the Reynolds- and Favre-averaged fields are functions only of the streamwise coordinate $x$ and time, while the fluctuating fields are functions of both the streamwise and spanwise coordinates and time.

\subsubsection{Evolution of the mole fraction profile $\langle X\rangle$}

Quantities previously introduced in the context of the analysis of Rayleigh-Taylor mixing by Youngs [103] and Cook and Dimotakis [25] are considered and adapted to the RichtmyerMeshkov instability here. First consider the mole fraction $X(x, y, t)$ averaged over the spanwise direction $y,\langle X\rangle(x, t)$. Consider two fluids with constant densities $\rho_{1}$ and $\rho_{2}>\rho_{1}$. The mole fraction $X$ defined in Eq. (157) varies from $X=0$ in the first fluid to $X=1$ in the second fluid, so that the mole fraction profile $\langle X\rangle(x, t)$ gives the relative distribution of mass of the two fluids within the mixing layer. If the two fluids are uniformly-distributed over the entire volume under consideration, as expected in complete homogeneous mixing, then $\langle X\rangle=0.5$.

To facilitate the comparison of the mole fraction profile at different times, the streamwise coordinate is recentered by the location of the midpoint between the bubble and spike position. The left column of Fig. 18 shows the recentered mole fraction profile at time intervals of $1 \mathrm{~ms}$ from $t=0$ to $t=17 \mathrm{~ms}$. The mole fraction profile increases from $\langle X\rangle=0$ in the air(acetone) mixture to $\langle X\rangle=1$ in $\mathrm{SF}_{6}$. The increasing width of the profile shows the spatial spreading of the mixing layer in time. The curves further display the varying distribution of mass inside the mixing layer prior to reshock. Initially, at $t=0 \mathrm{~ms}$ the profile is monotonically-increasing as the initial interface is slightly diffused. After the initial passage of the shock, the profile increases in width and becomes non-monotonic with a peak developing within the air(acetone) gas mixture, and moving to the left and increasing in magnitude in time. This is due to spikes of $\mathrm{SF}_{6}$ penetrating into the air(acetone), forming the characteristic roll-ups. These roll-ups are a consequence of the entrainment of additional $\mathrm{SF}_{6}$ within the air(acetone) mixture. The bubbles of air(acetone) "rise" in the $\mathrm{SF}_{6}$, causing an overall decrease in $\langle X\rangle$ in the $\mathrm{SF}_{6}$. Reshock compresses the averaged mole fraction, as shown between $t=6$ and $t=7 \mathrm{~ms}$. The width of the mixed layer increases rapidly following reshock. Reshock also generates additional structure, which is reflected in the distribution of $\langle X\rangle$ (now displaying several localized peaks). For later times, the growth of the mixing layer slows and the localized peaks decrease in magnitude, and $\langle X\rangle$ begins to approach 0.5 , indicating a well-mixed distribution of mass within the layer.

The right column of Fig. 18 shows the mole fraction profile $\langle X\rangle$ with the recentered streamwise coordinate rescaled by the mixing layer width $h$. This rescaling adjusts the profiles so that the rescaled edges of the mixing layer are identical at different times, and results in a loss of information regarding the width of the layer, but facilitates the investigation of the mechanisms of the mixing process and the distribution of mixed mass within the layer. The figures at early times indicate the transfer of mass between the bubbles and spikes, causing the non-monotonic profiles noted above. Prior to reshock, the profile evolves smoothly in space and time. Reshock causes a fundamental change in the distribution of mass with additional 
$\langle X\rangle$ recentered
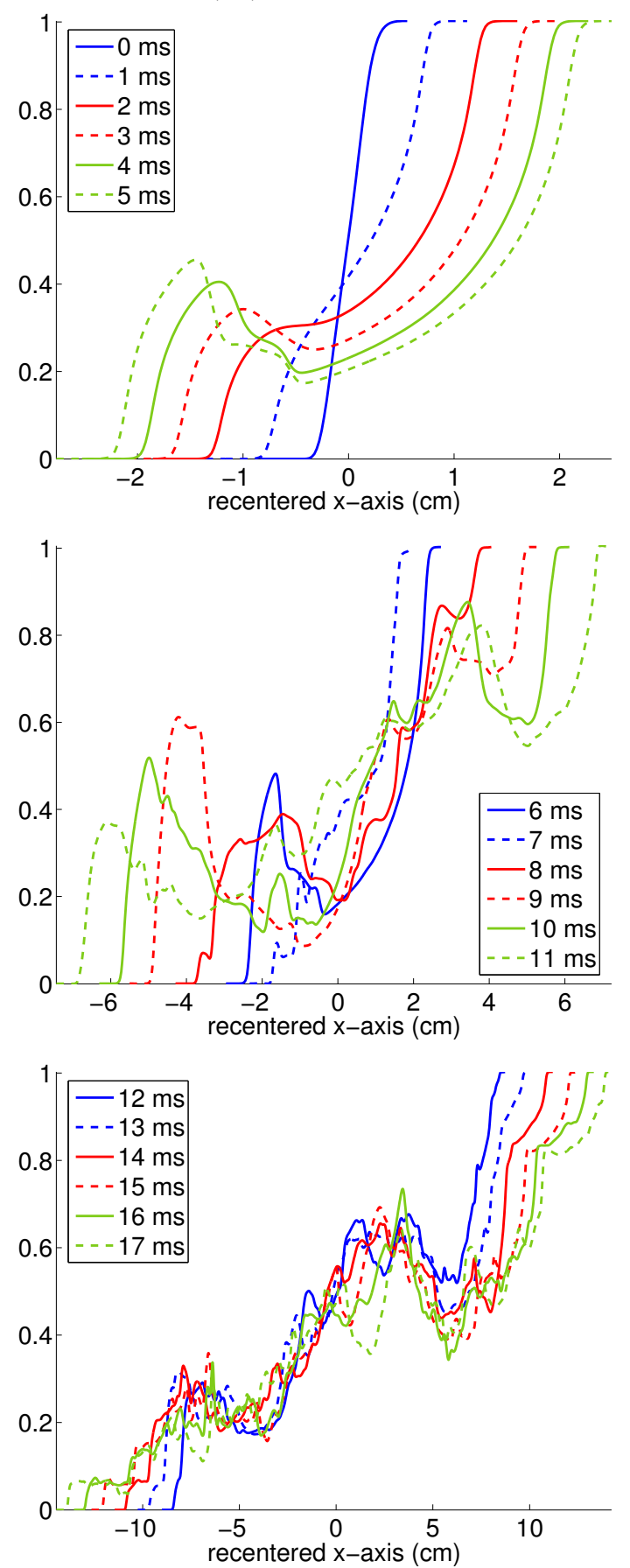

$\langle X\rangle$ recentered and rescaled
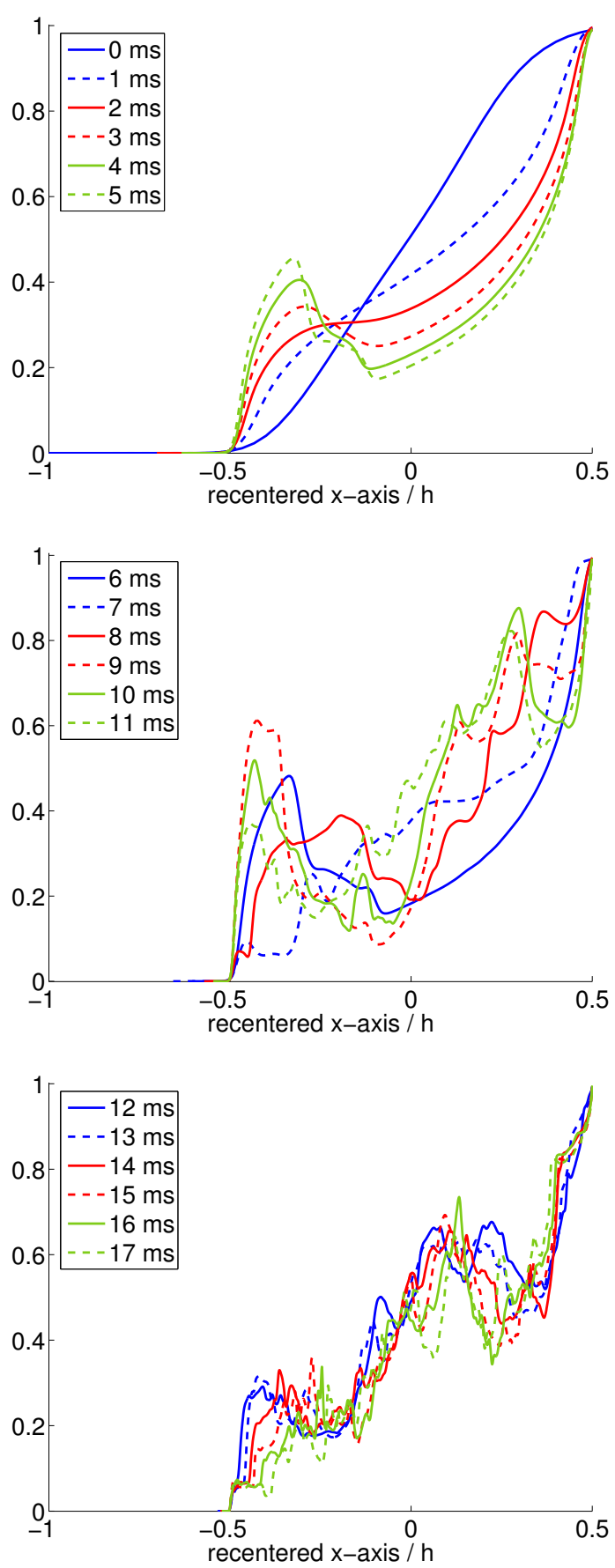

Figure 18: Time-evolution of the mole fraction profile $\langle X\rangle$ with the $x$-axis recentered (left column), and $\langle X\rangle$ with the $x$-axis recentered and rescaled by the total mixing layer width $h$ (right column). 
structures forming, as reflected in the more complex structure of the profile than at earlier times. For late times, the profile begins to collapse and exhibit increased oscillations resulting from the break-up of structures.

\subsubsection{Evolution of the profiles of the averaged chemical product from a fast kinetic reaction $\left\langle X_{p}\right\rangle, X_{p}(\langle X\rangle)$ and of the mixing fraction $\xi$}

A quantitative measure of mixing can be defined as follows. Suppose that the two fluids undergo a fast kinetic reaction, so that the amount of product produced (see Koochesfahani and Dimotakis [50] and Cook and Dimotakis [25]) is

$$
X_{p}(x, y, t)=\left\{\begin{array}{ll}
\frac{X}{X_{s}} & \text { for } X \leq X_{s} \\
\frac{1-X}{1-X_{s}} & \text { for } X>X_{s}
\end{array},\right.
$$

where $X_{s}$ is a parameter chosen to be $X_{s}=1 / 2$ here (indicating that the product is composed of one mole of each reactant), and is limited by the amount of reactant (either the heavy or the light fluid). The profile of the averaged product mole fraction $\left\langle X_{p}\right\rangle(x, t) \in[0,1]$ provides information on how well mixed the two reactants are. The maximum amount of reactant mole is $X_{p}(\langle X\rangle) \geq\left\langle X_{p}\right\rangle$ if the two reactants were homogeneously mixed in the spanwise direction. The ratio

$$
\xi(x, t)=\frac{\left\langle X_{p}\right\rangle}{X_{p}(\langle X\rangle)},
$$

which is between zero and unity, also locally characterizes how well mixed the two fluids are. If the fluids were completely and homogeneously mixed, $\left\langle X_{p}\right\rangle=X_{p}(\langle X\rangle)$, so that $\xi(x, t)=1$.

The left column of Fig. 19 shows the time-evolution of the averaged mole profile $\left\langle X_{p}\right\rangle$. The initial diffusion layer is well-mixed, with a rapid decrease in $\left\langle X_{p}\right\rangle$ between $t=0$ and 1 ms, as expected; $\left\langle X_{p}\right\rangle$ broadens and rapidly develops sharp cusps at the edges of the mixing layer $x / h(t) \approx \pm 0.5$ as the spikes of heavy fluid penetrate into the lighter fluid and bubbles of the light fluid "rise" in the lighter fluid (and the two fluids become less mixed). At these very early times in the instability evolution, $\left\langle X_{p}\right\rangle$ is nearly-symmetric, with values ranging from $\approx 0.05-0.06$ over most of the layer. A pronounced asymmetry develops for $t>2 \mathrm{~ms}$ : as the roll-ups form, a further peak within the air(acetone) mixture develops, corresponding to wellmixed fluid in the cores. Reshock significantly increases the product mole $\left\langle X_{p}\right\rangle$, as additional fine-scale structures form; $\left\langle X_{p}\right\rangle$ exhibits significant oscillations following reshock, which persist to late times. At late times the fluids tend to be more mixed and larger peaks develop in the $\mathrm{SF}_{6}$, corresponding to the arrival of multiple reflected waves that produce increased mixing at one end of the mixing layer. The gases tend to be less mixed in the region closer to the air(acetone) than in the region closer to the $\mathrm{SF}_{6}$.

The right column of Fig. 19 shows the mole fraction of the chemical product if the two fluids were completely mixed, $X_{p}(\langle X\rangle)$. This quantity measures the fraction of the maximum amount of product $X_{p}$ observed if the two reactants were completely mixed $\langle X\rangle$. This quantity indicates the maximum possible value of $\left\langle X_{p}\right\rangle$. At early times, $X_{p}(\langle X\rangle)$ is peaked at $x / h=0$. Like $\left\langle X_{p}\right\rangle, X_{p}(\langle X\rangle)$ exhibits a complex spatial structure, with large oscillations following reshock.

The left column of Fig. 21 shows the ratio $\xi$ defined in Eq. (175). This quantity constitutes a local mixing fraction characterizing how well the two fluids are mixed in the layer. For $t>0$, $\xi$ decreases rapidly, attaining its largest values near the edges of the mixing layer. At early times $t<2 \mathrm{~ms}, \xi \approx 0.1$ across most of the layer. For $t>2 \mathrm{~ms}, \xi$ develops peaks on the 

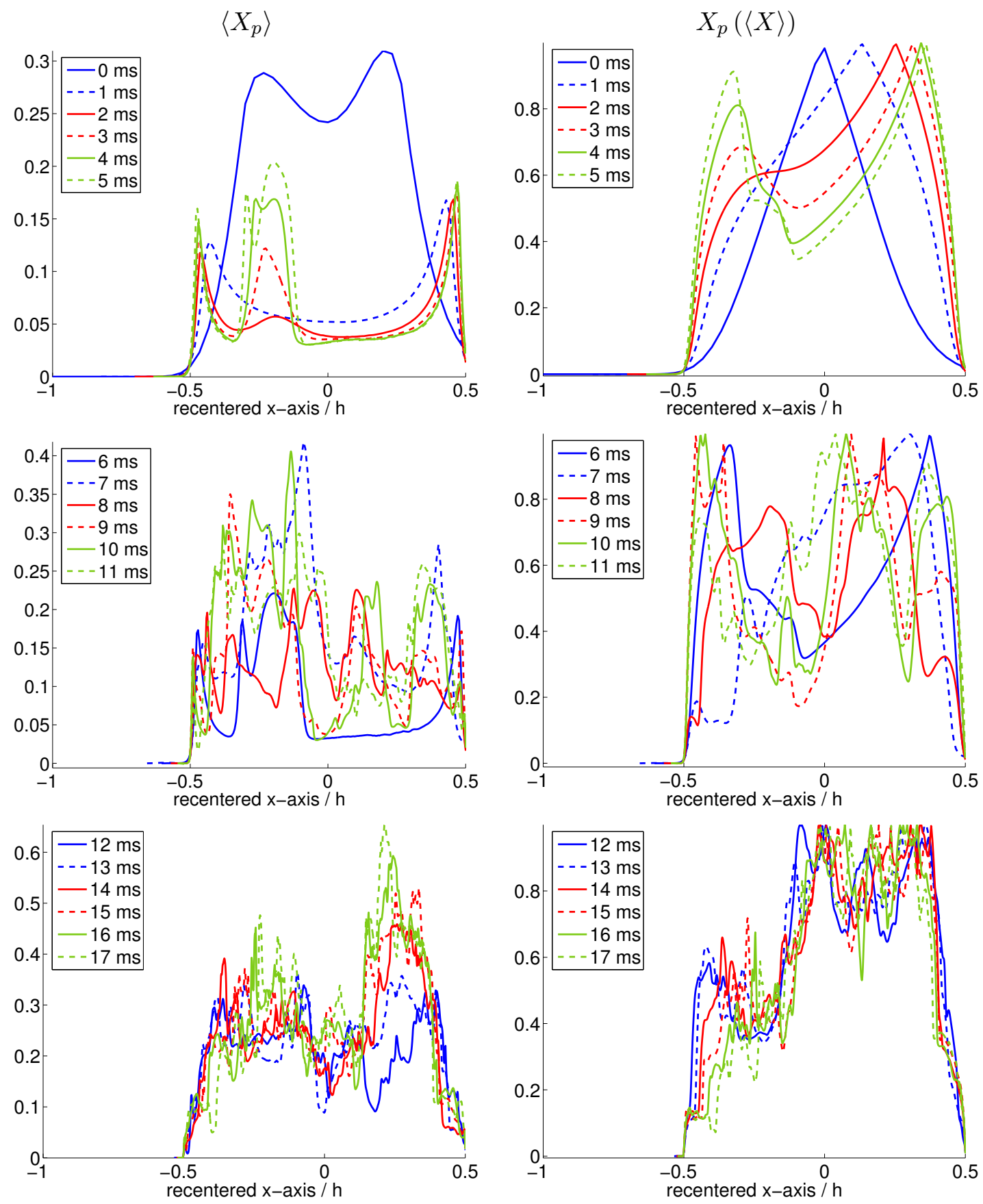

Figure 19: Time-evolution of the product mole fraction profile $\left\langle X_{p}\right\rangle$ (left column) and the product mole fraction if the fluids were homogeneously mixed $X_{p}(\langle X\rangle)$ with the $x$-axis recentered and rescaled by the total mixing layer width $h$ (right column). 
air(acetone) side. Reshock amplifies $\xi$ and induces strong oscillations across the mixing layer. At times $t>12 \mathrm{~ms}, \xi$ is strongly peaked on the air(acetone) side.

\subsubsection{Evolution of the profiles of the volume fractions $\left\langle f_{1}\right\rangle,\left\langle f_{2}\right\rangle$ and of the mixing fractions $\xi$ and $\theta$}

The volume fractions (134) can be averaged over the spanwise direction to give the volume fraction profiles $\left\langle f_{r}\right\rangle(x, t)$. Figure 20 shows the volume fraction profiles $\left\langle f_{1}\right\rangle$ and $\left\langle f_{2}\right\rangle$, where 1 and 2 correspond to the air(acetone) and $\mathrm{SF}_{6}$, respectively. Note that the spatio-temporal evolution of $\left\langle f_{2}\right\rangle$ is very similar to that of $\langle X\rangle$.

The local molecular mixing fraction $\theta(x, t)$ is obtained from the averaged volume fractions and the average of their product $[102,103]$

$$
\theta(x, t)=\frac{\left\langle f_{1} f_{2}\right\rangle}{\left\langle f_{1}\right\rangle\left\langle f_{2}\right\rangle}
$$

The mixing fractions $\xi$ and $\theta$ are shown in the left and right columns of Fig. 21, respectively. Note the similarity between $\theta$ and $\xi$ due to the fact that both quantities are a measure of mixing, indicating that both mechanisms - the fast kinetic reaction and the diffusion process characterized by $\theta$-provide a very similar qualitative description of mixing. Note that this 'molecular mixing' is induced by stirring and by the numerical diffusion present in the algorithm, rather than by molecular processes.

\subsection{Time-evolution of mixing fractions and of volume-averaged quantities}

The time-evolution of global mixing fractions such as the production quantities $P_{t}$ and $P_{m}$, and the mixing fractions $\Xi$ and $\Theta$ are presented here. The volume-averaged streamwise and spanwise velocity components, and pressure are also investigated.

The lengthscale $W$ is shown in the top right of Fig. 22, and qualitatively resembles the evolution of the mixing layer width $h$ shown in the top left of Fig. 22. Note that these lengthscales do not appear to exhibit a simple power-law growth at late times.

\subsubsection{Evolution of $P_{t}, P_{m}, \Theta$, and $\Xi$}

From the product mole fraction $X_{p}$, the total chemical product $P_{t}$ is obtained by integrating $\left\langle X_{p}\right\rangle$ over the mixing layer width,

$$
P_{t}(t)=\int_{a_{s}(t)}^{a_{b}(t)}\left\langle X_{p}\right\rangle \mathrm{d} x
$$

The total chemical product $P_{t}$ can be compared with the maximum amount of chemical product

$$
\begin{aligned}
P_{m}(t) & =\int_{a_{s}(t)}^{a_{b}(t)} X_{p}(\langle X\rangle) \mathrm{d} x \\
& \geq P_{t}(t)
\end{aligned}
$$

measuring the product obtained if both reactants were homogeneously mixed.

The time-evolution of $P_{t}$ and $P_{m}$ is shown in the middle row of Fig. 22. Before reshock, $P_{t}$ increases, indicating an increase in mixing, while $P_{m}$ decreases. The decrease in $P_{m}$ indicates that the maximum molar product decreases due to the presence of the bubble which 
$\left\langle f_{1}\right\rangle$
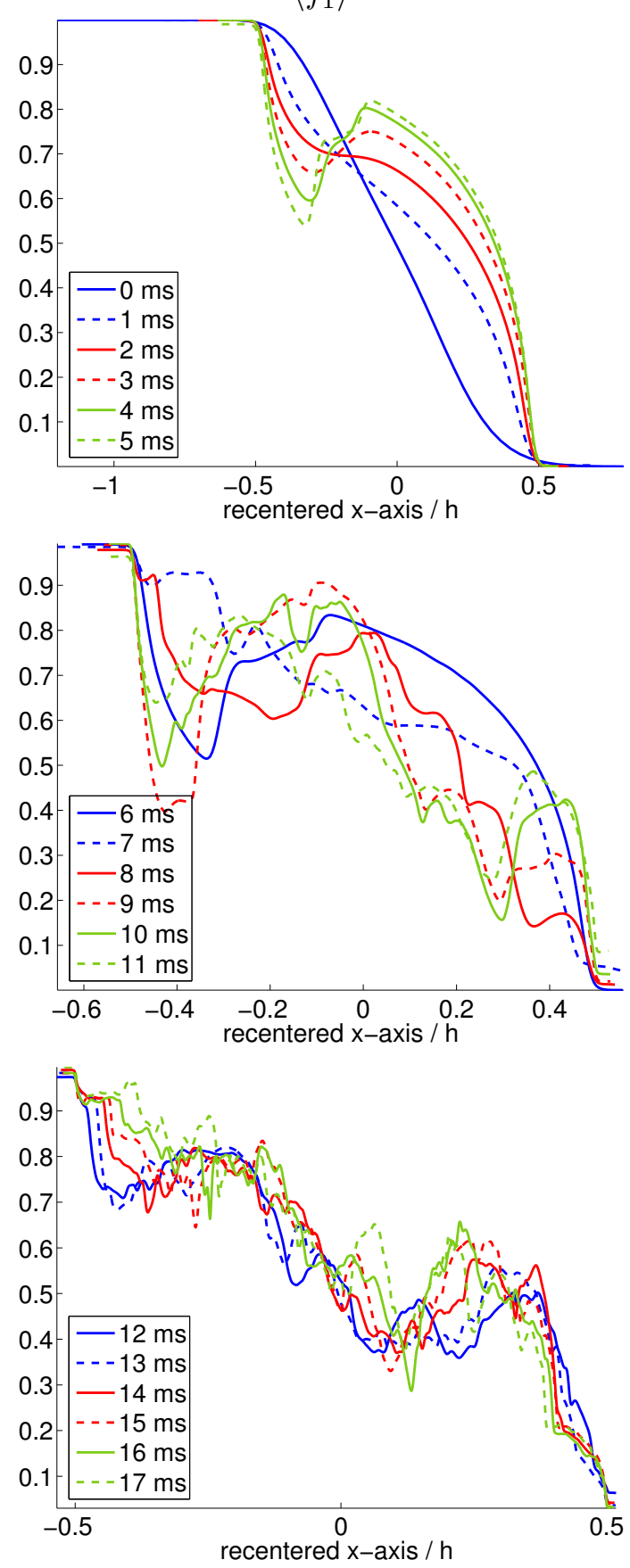

$\left\langle f_{2}\right\rangle$
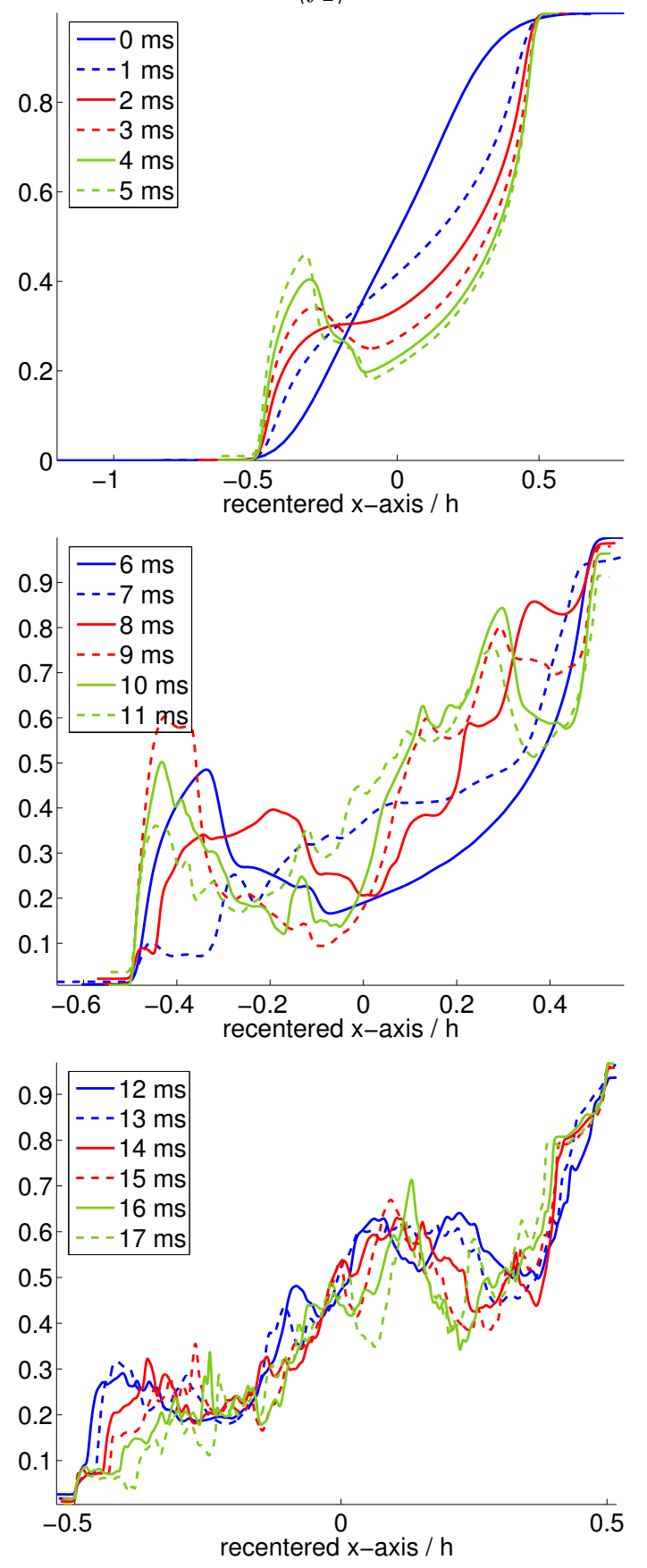

Figure 20: Time-evolution of the volume fractions $\left\langle f_{1}\right\rangle$ (left column) and $\left\langle f_{2}\right\rangle$ (right column) with the $x$-axis recentered and rescaled by the total mixing layer width $h$. 

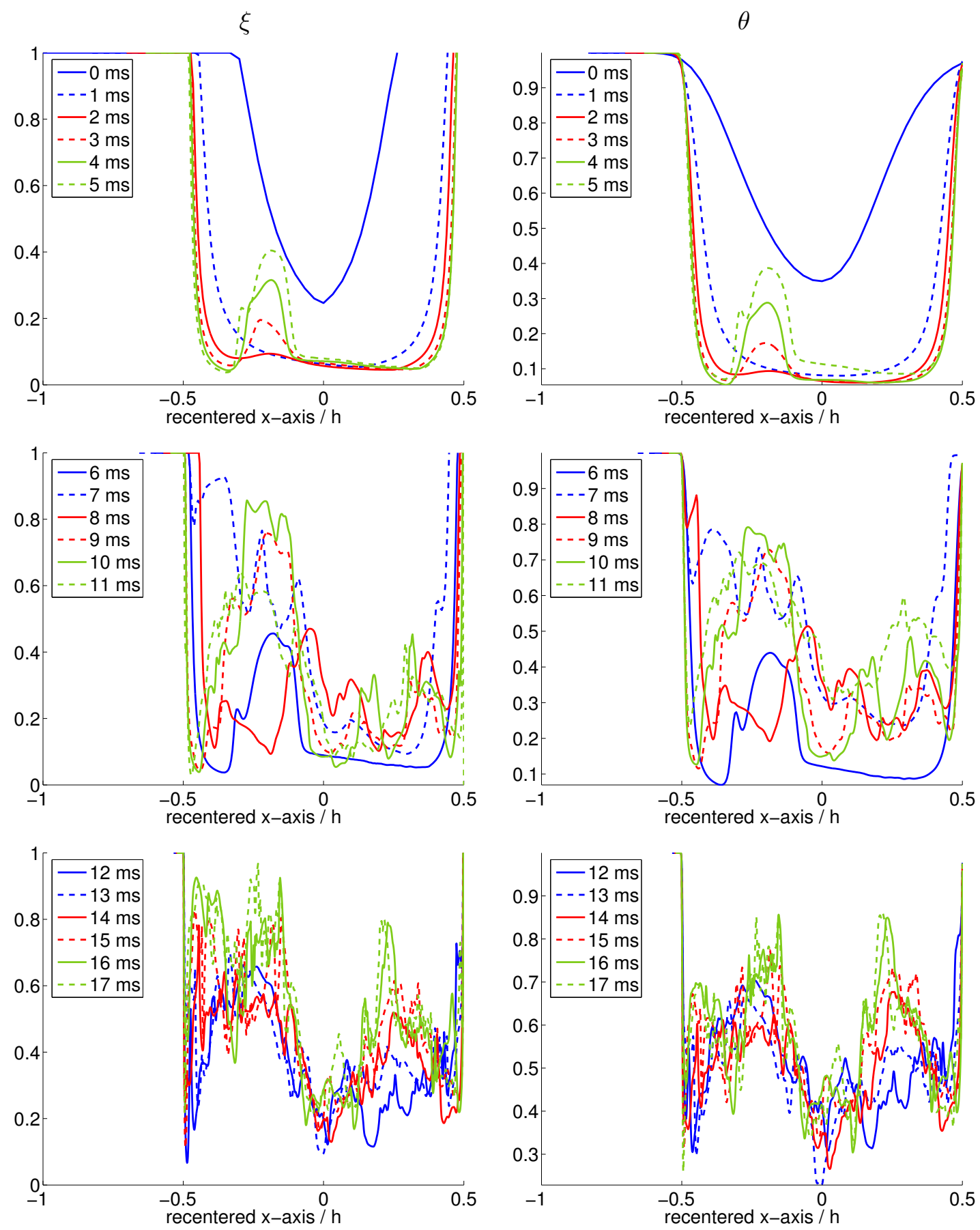

Figure 21: Time-evolution of the molecular mixing fractions $\xi$ (left column) and $\theta$ (right column) with the $x$-axis recentered and rescaled by the total mixing layer width $h$. 
creates large, unmixed structures within the mixing layer. During reshock, the mixing layer is compressed, inducing additional mixing as measured by $P_{t}$. Following reshock, $P_{t}$ increases rapidly, indicating significantly increased mixing. As the mixing progresses, large unmixed structures form, causing the decrease in $P_{m}$.

The ratio of the total and maximum chemical product gives the mixing fraction [25]

$$
\Xi(t)=\frac{P_{t}(t)}{P_{m}(t)},
$$

with increasing values of $\Xi$ signifying more complete mixing. From the local molecular mixing fraction $\theta$, another global molecular mixing fraction can be defined as [102]

$$
\Theta(t)=\frac{\int_{a_{s}(t)}^{a_{b}(t)}\left\langle f_{1} f_{2}\right\rangle \mathrm{d} x}{\int_{a_{s}(t)}^{a_{b}(t)}\left\langle f_{1}\right\rangle\left\langle f_{2}\right\rangle \mathrm{d} x},
$$

with increasing values of $\Theta$ also corresponding to more complete mixing.

The time-evolution of the mixing fractions $\Theta$ and $\Xi$ is also shown in the middle row of Fig. 22. As was observed for $\xi$ and $\theta$ in Fig. 21, the mixing fractions $\Xi$ and $\Theta$ give qualitatively similar information. As the fluids mix following the initial shock passage, the mixing fractions increase. A spike is observed during reshock as the mixing layer is compressed and the overall mixing is increased. Following reshock, the mixing fractions increase at a faster rate than before reshock, indicating that reshock increases mixing.

\subsubsection{Evolution of the volume-averaged velocity components and pressure}

The time-evolution of the volume-averaged streamwise and spanwise velocities, and pressure are presented here. In two dimensions, define the volume average of $\phi(x, y, t)$ by

$$
\langle\phi\rangle_{x y}(t)=\frac{1}{h(t) L_{y}} \int_{a_{s}(t)}^{a_{b}(t)} \int_{0}^{L_{y}} \phi(x, y, t) \mathrm{d} y \mathrm{~d} x .
$$

The volume-averaged streamwise and spanwise velocity components $\langle u\rangle_{x y}$ and $\langle v\rangle_{x y}$, respectively, are shown in the bottom row of Fig. 22 as a function of time. As expected, $\langle v\rangle_{x y}=0$ for all time. Note that $\langle u\rangle_{x y}$ is the velocity of the interface, which rapidly decreases at reshock; $\langle u\rangle_{x y}$ changes sign at reshock and remains nearly constant over $t \approx 6.5-10.5 \mathrm{~ms}$, indicating an inversion of the direction of the mean motion of the mixing layer, as well as the effect of the reflected rarefaction wave. Note that $\langle u\rangle_{x y} \rightarrow 0$ at late times, as the reflected waves following reshock decelerate the interface and eventually bring it to rest.

The volume-averaged pressure $\langle p\rangle_{x y}$ is also shown in the bottom row of Fig. 22 as a function of time. The average pressure reflects the effect of the reshock (which significantly increases the pressure during compression) and of the rarefaction wave (which decreases the pressure). A nearly constant value of $\langle p\rangle_{x y}$ is maintained over $t \approx 6.5-10.5 \mathrm{~ms}$. The temporal evolution of both $\langle u\rangle_{x y}$ and $\langle p\rangle_{x y}$ clearly show the interaction of the reflected waves with the mixing layer.

\subsection{Time-evolution of the fluctuating kinetic energy, fluctuating enstrophy, density variance, and pressure variance spectra}

The time-evolution of the spectra corresponding to the fluctuating kinetic energy, fluctuating enstrophy, density variance, and pressure variance are investigated here. An examination of 

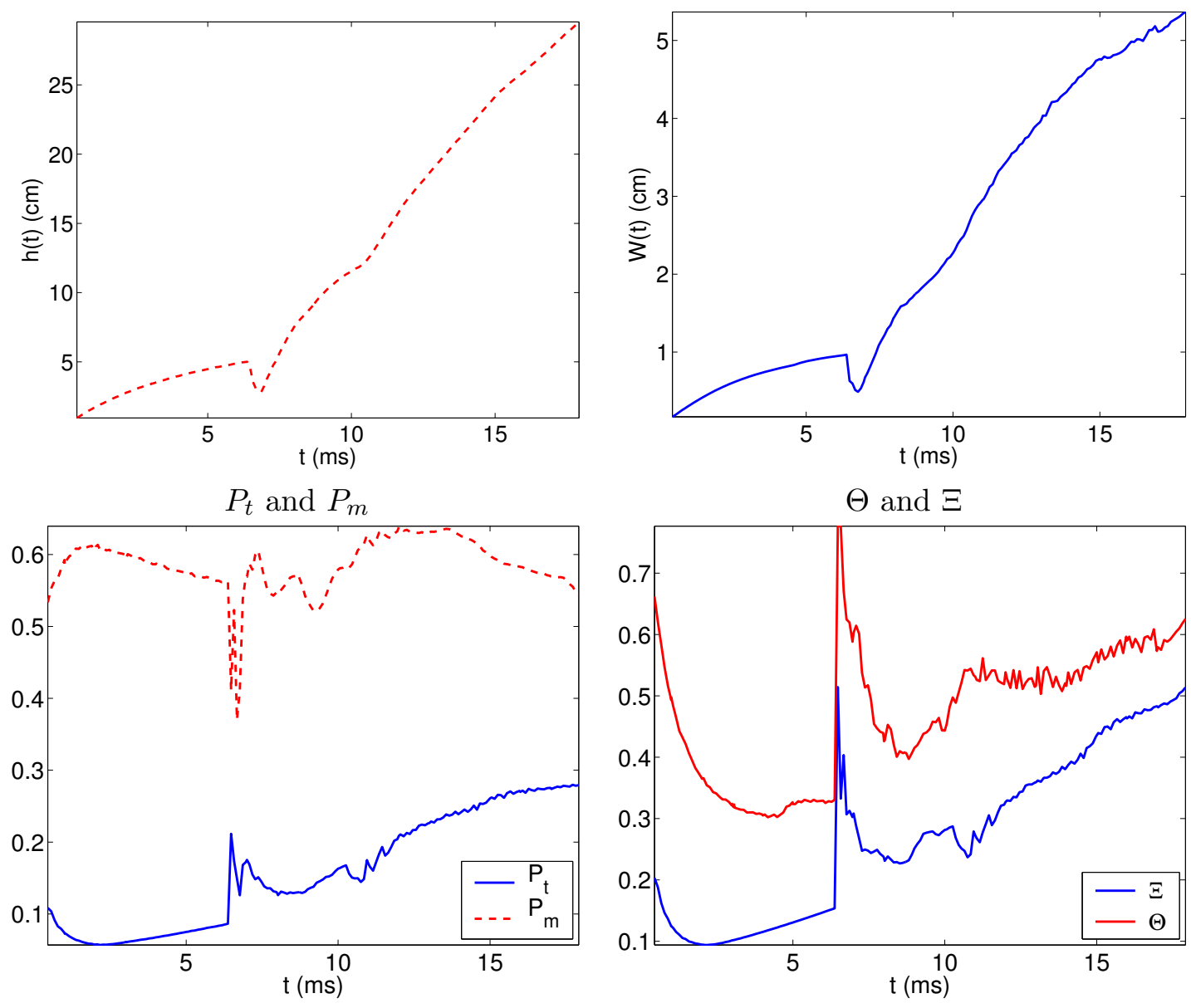

$\langle u\rangle_{x y}$ and $\langle v\rangle_{x y}$
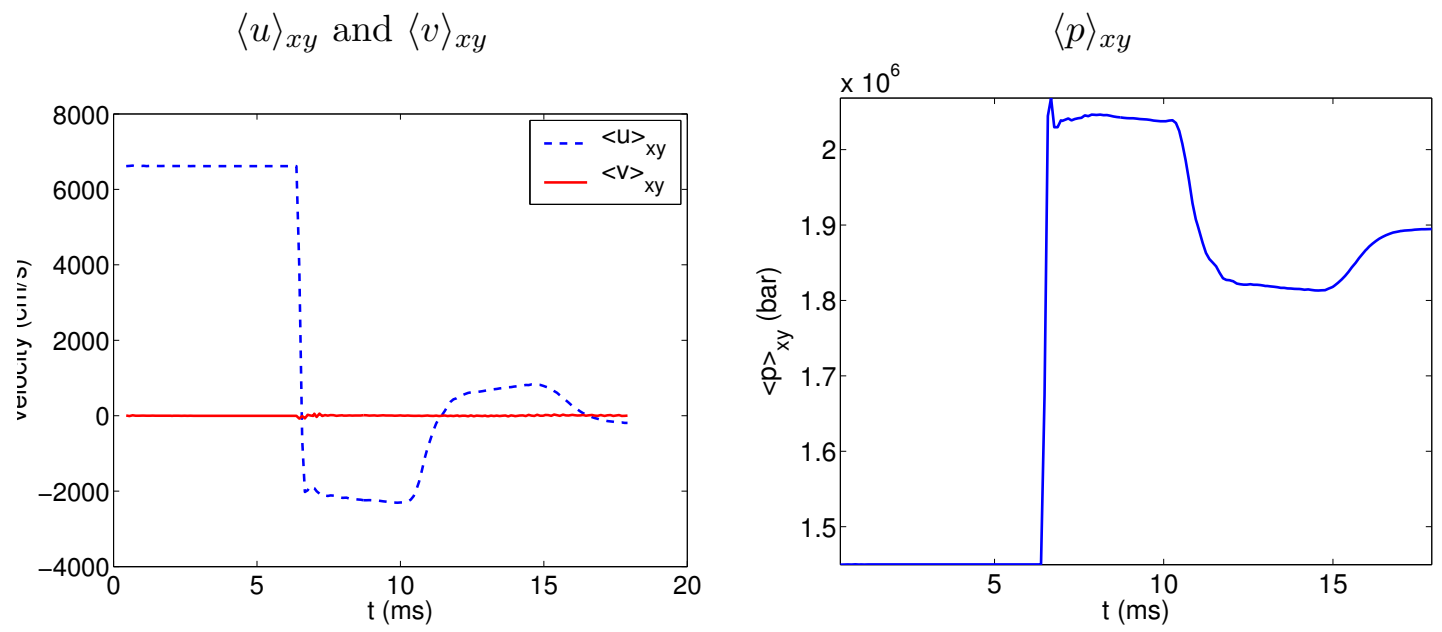

Figure 22: Time-evolution of the mixing layer width as measured by $h(t)$ and $W(t)$ in the top row. The mixing fractions $P_{t}, P_{m}, \Theta$, and $\Xi$ are shown in the middle row. The volume-averaged streamwise and spanwise velocities $\langle u\rangle_{x y}$ and $\langle v\rangle_{x y}$, and the volume-averaged pressure $\langle p\rangle_{x y}$ are shown in the bottom row. 
these spectra provides information on the growth of different scales of the flow, as well as on the energy transfer process among scales. Summarized here is the general procedure used in the present investigation to determine the spectrum of a quantity $\phi(x, y, t)$ defined in the mixing layer $x \in\left[a_{s}, a_{b}\right]$. Typically, $\phi$ is a Reynolds fluctuation $\phi^{\prime}$ defined in Eq. (171) or a Favre fluctuation $\phi^{\prime \prime}$ defined in Eq. (173). The spectral analysis used here adapts the formulations of Lesieur et al. [61] and Mügler and Gauthier [75, 76] applied to inhomogeneous flows with a direction of statistical-homogeneity (periodicity). The fundamental procedures of Fourier analysis are reviewed elsewhere [36, 17, 83].

First, the Fourier transform is taken in the periodic spanwise direction to obtain

$$
\widehat{\phi}(k, x, t)=\frac{1}{2 \pi} \int_{-\infty}^{\infty} \phi(x, y, t) \exp (-i k y) \mathrm{d} y .
$$

Numerically, the discrete Fourier transform of $\phi(x, y, t)$ is taken in the periodic $y$-direction to obtain the one-dimensional spectrum,

$$
\widehat{\phi}\left(k_{n}, x, t\right)=\frac{1}{N_{y}} \sum_{n=0}^{N_{y}-1} \phi_{n}(x, t) \exp \left(-i k_{n} \Delta y\right),
$$

where $n=0,1, \ldots, N_{y}$, and $N_{y}$ is the number of grid points in the $y$-direction with uniform grid spacing $\Delta y$, and

$$
k_{n}=\frac{2 \pi n}{N_{y} \Delta y}
$$

is the discrete wavenumber ( $n . b .$, in the subsequent analysis, the units of $k$ are $\mathrm{cm}^{-1}$ ). Such a spectrum is appropriate for a two-dimensional flow having one direction of statistical-homogeneity, in which periodic boundary conditions are assumed.

The energy associated with each Fourier mode $k$ is obtained by averaging over the extent of the mixing layer to obtain the one-dimensional energy spectrum of the quantities $\phi$ and $\psi$,

$$
E_{\phi \psi}(k, t)=\frac{1}{2 h(t)} \int_{a_{s}(t)}^{a_{b}(t)}\left[\widehat{\phi}(k, x, t) \widehat{\psi}(k, x, t)^{*}+\widehat{\phi}(k, x, t)^{*} \widehat{\psi}(k, x, t)\right] \mathrm{d} x
$$

where $h$ is given by Eq. (159) and $*$ indicates complex conjugation. Numerically, this integration over the mixing layer width is performed using the trapezoidal rule. In the results presented below, all modes above the Nyquist wavenumber (127) are neglected [83]. The spectrum $E_{\phi \psi}(k, t)$ provides information on the energy content of all of the scales present in the statistical correlation between $\phi$ and $\psi$ as a function of time. This constitutes a local analysis, as modal information is obtained within the mixing layer as a function of scale. The characteristic scale of a structure with wavenumber $k$ is $\ell \sim 1 / k$.

The spatial profiles of the one-dimensional spectral density

$$
E_{\phi \psi}\left(k_{n}, x, t\right)=\frac{\widehat{\phi}\left(k_{n}, x, t\right) \widehat{\psi}\left(k_{n}, x, t\right)^{*}+\widehat{\phi}\left(k_{n}, x, t\right)^{*} \widehat{\psi}\left(k_{n}, x, t\right)}{2}
$$

can also be considered, in addition to the spatially-integrated spectra (186). The usual energy spectrum corresponds to $\psi=\phi$ :

$$
E_{\phi \phi}\left(k_{n}, x, t\right)=\left|\widehat{\phi}\left(k_{n}, x, t\right)\right|^{2}
$$



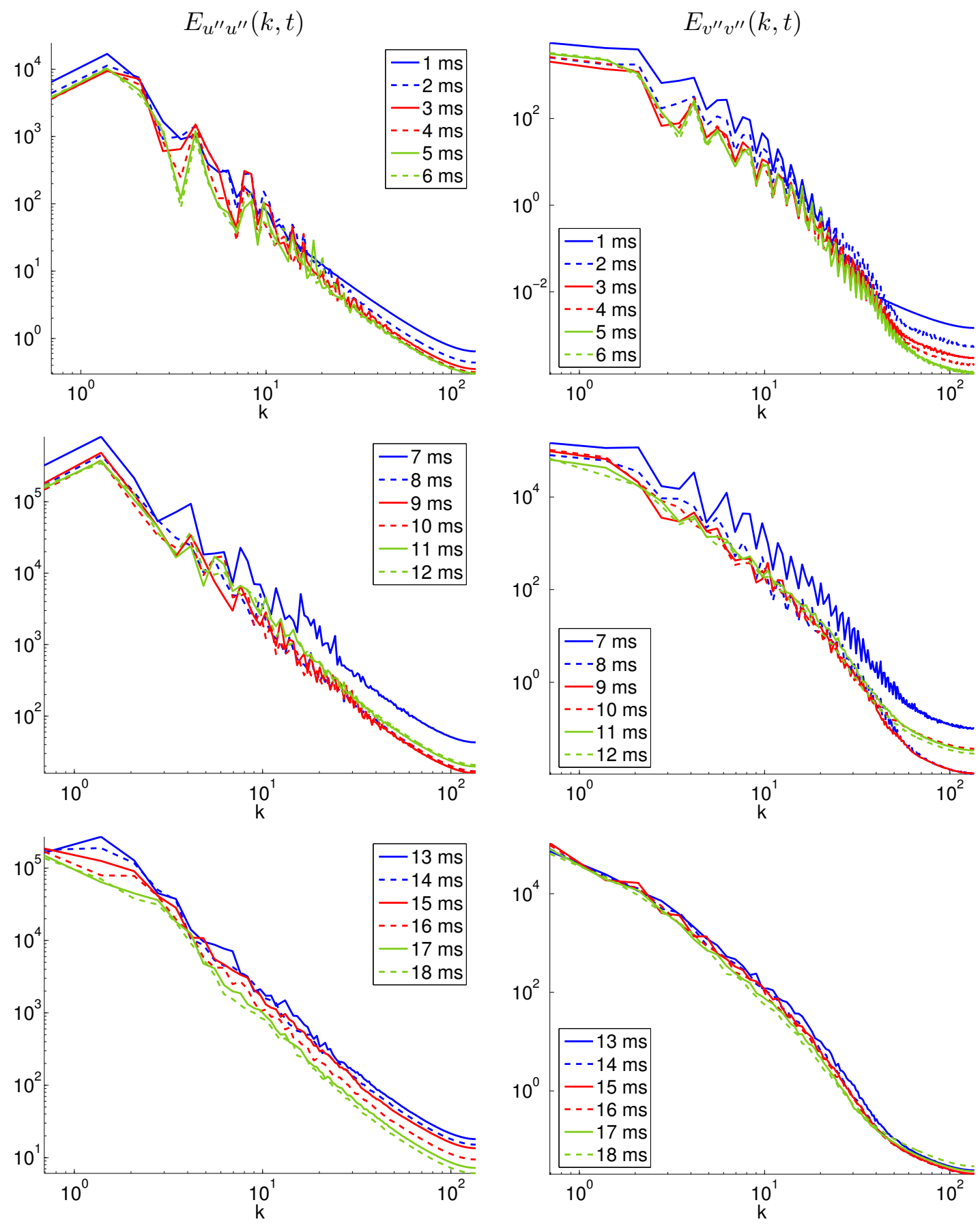

Figure 23: Time-evolution of the streamwise and spanwise fluctuating kinetic energy spectra $E_{u^{\prime \prime} u^{\prime \prime}}(k, t)$ (left column) and $E_{v^{\prime \prime} v^{\prime \prime}}(k, t)$ (right column), respectively. 

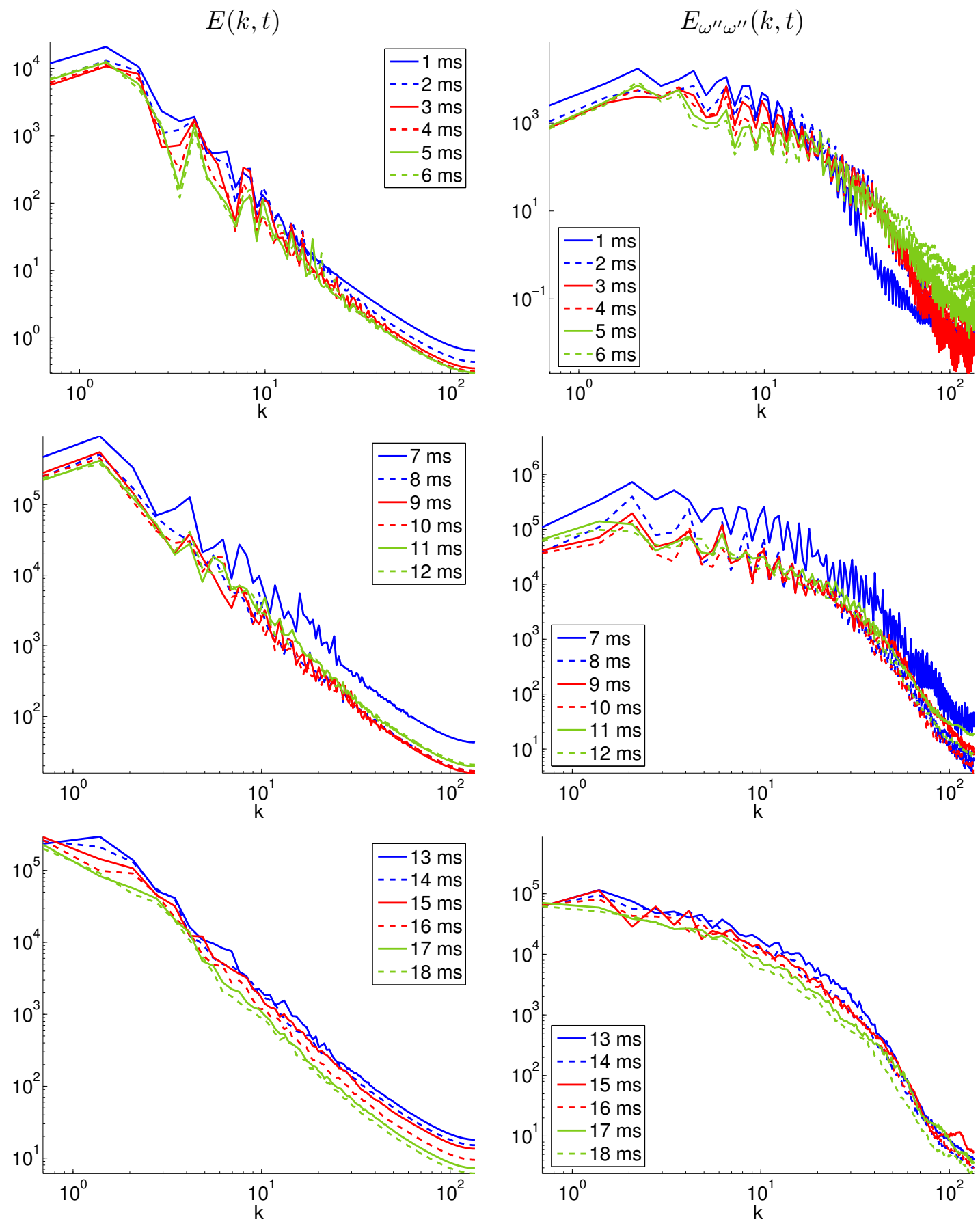

Figure 24: Time-evolution of the (total) fluctuating kinetic energy spectrum $E(k, t)=$ $E_{u^{\prime \prime} u^{\prime \prime}}(k, t)+E_{v^{\prime \prime} v^{\prime \prime}}(k, t)$ (left column) and fluctuating enstrophy spectrum $E_{\omega^{\prime \prime} \omega^{\prime \prime}}(k, t)$ (right column) 
with

$$
E_{\phi \phi}\left(k_{n}, t\right)=\frac{1}{h(t)} \int_{a_{s}(t)}^{a_{b}(t)}\left|\widehat{\phi}\left(k_{n}, x, t\right)\right|^{2} \mathrm{~d} x .
$$

Figures 23 and 24 show the time-evolution of the streamwise and spanwise fluctuating kinetic energy spectra per unit volume $E_{u^{\prime \prime} u^{\prime \prime}}(k, t)$ and $E_{v^{\prime \prime} v^{\prime \prime}}(k, t)$, respectively, and the total fluctuating kinetic energy spectrum per unit volume

$$
E(k, t)=E_{u^{\prime \prime} u^{\prime \prime}}(k, t)+E_{v^{\prime \prime} v^{\prime \prime}}(k, t) .
$$

Oscillations are present in the spectra for small and intermediate wavenumbers $k$ at early times, and following reshock. These oscillations are damped out at late times, as indicated by the smoothly decaying spectra at large $t$. Reshock induces a jump in the energy spectra between $t=6 \mathrm{~ms}$ and $t=7 \mathrm{~ms}$ at all scales of the flow by exciting a wide spatial range of fluctuations, thereby imparting additional energy into the mixing layer. For times $t>8 \mathrm{~ms}$, the energy spectra appear to decay very slowly. Note that $E_{u^{\prime \prime} u^{\prime \prime}}(k, t)$ is peaked at $k \approx 1.5$, while the peak of $E_{v^{\prime \prime} v^{\prime \prime}}(k, t)$ corresponds to the largest scale of the flow. As expected, there is more energy content in the streamwise velocity fluctuations than in the spanwise velocity fluctuations. For the same reason, the evolution of $E(k, t)$ is dominated by that of $E_{u^{\prime \prime} u^{\prime \prime}}(k, t)$. There is no apparent power-law scaling of the spectra over the limited range of scales supported by the modest grid resolution. The spectra turn up slightly at large wavenumbers due to aliasing error.

The enstrophy density is

$$
\Omega(x, y, t)=\frac{|\omega(x, y, t)|^{2}}{2} .
$$

The volume integral of the enstrophy density yields the enstrophy

$$
\Omega(t)=\iint \Omega(x, y, t) \mathrm{d} x \mathrm{~d} y
$$

The time-evolution of the fluctuating enstrophy spectrum per unit volume $E_{\omega^{\prime \prime} \omega^{\prime \prime}}(k, t)$ is shown in Fig. 24. In a homogeneous flow, the enstrophy is related to the kinetic energy spectrum by $E_{\omega^{\prime \prime} \omega^{\prime \prime}}(k, t)=k^{2} E(k, t)$, so that the peak of the enstrophy spectrum is weighted toward smaller scales than that of the kinetic energy spectrum. The enstrophy spectrum is less steep than the fluctuating kinetic energy spectrum. As in the case of $E(k, t)$, reshock primarily amplifies the enstrophy spectrum but does not change its shape. A slow decay of the enstrophy spectrum is also observed for late times. Before and immediately after reshock, the fluctuating enstrophy spectrum is more oscillatory than the fluctuating kinetic energy spectrum, particularly at large wavenumbers.

To study the spectral anisotropy of the velocity components, the time-evolution of the ratio of the streamwise and spanwise fluctuating kinetic energy spectra $E_{u^{\prime \prime} u^{\prime \prime}}(k, t) / E_{v^{\prime \prime} v^{\prime \prime}}(k, t)$ is shown in Fig. 25. Spectral isotropy is achieved if all scales contain the same amount of energy, i.e., if the ratio approaches unity. As seen in the figure, significant spectral anisotropy exists in the intermediate and small scales, with the ratios attaining a nearly constant value at large wavenumbers. It is interesting to note that a sharp increase in the ratio of the streamwise and spanwise fluctuating kinetic energy is observed following the arrival of reshock and the interaction with the reflected rarefaction wave. This is expected as the arrival of the waves excites velocity fluctuations in all directions, therefore reducing the ratio. However, as the instability further evolves following the interaction with the waves, the fluctuations in the 

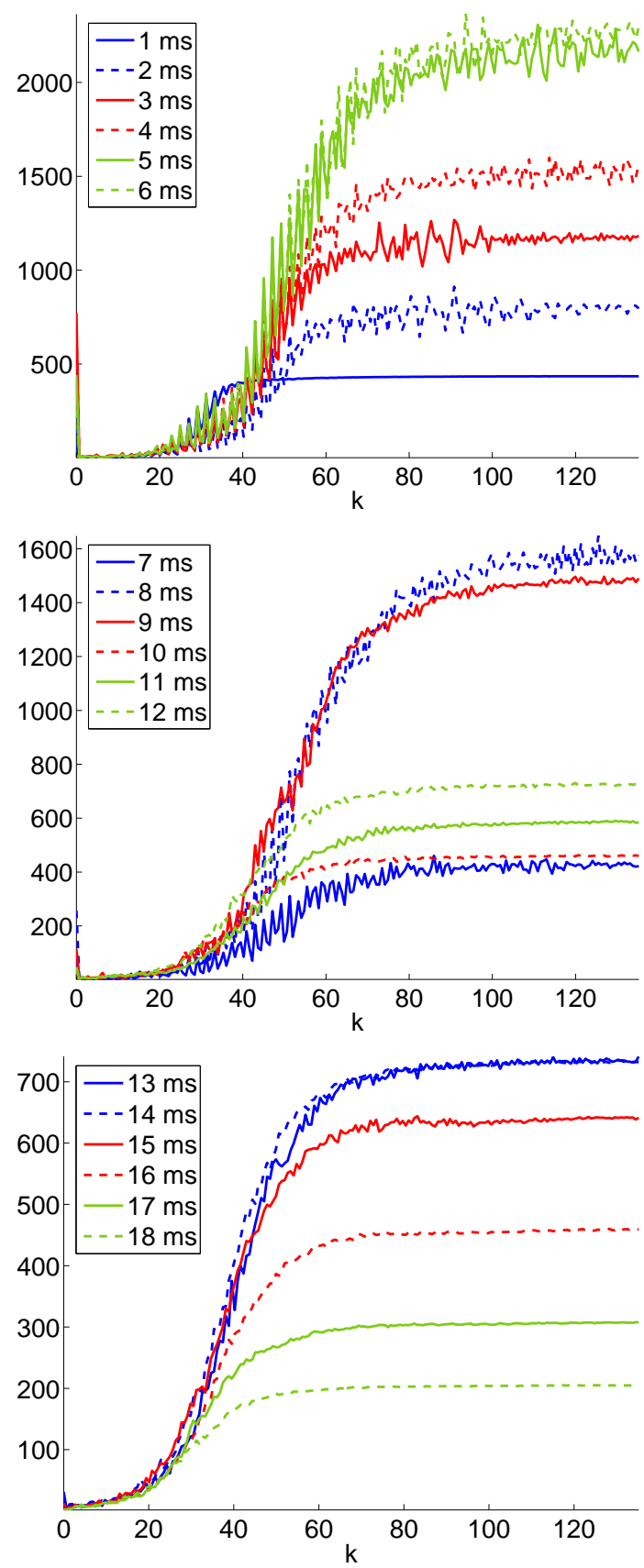

Figure 25: Time-evolution of the ratio of the streamwise and spanwise fluctuating kinetic energy spectra $E_{u^{\prime \prime} u^{\prime \prime}}(k, t) / E_{v^{\prime \prime} v^{\prime \prime}}(k, t)$. 

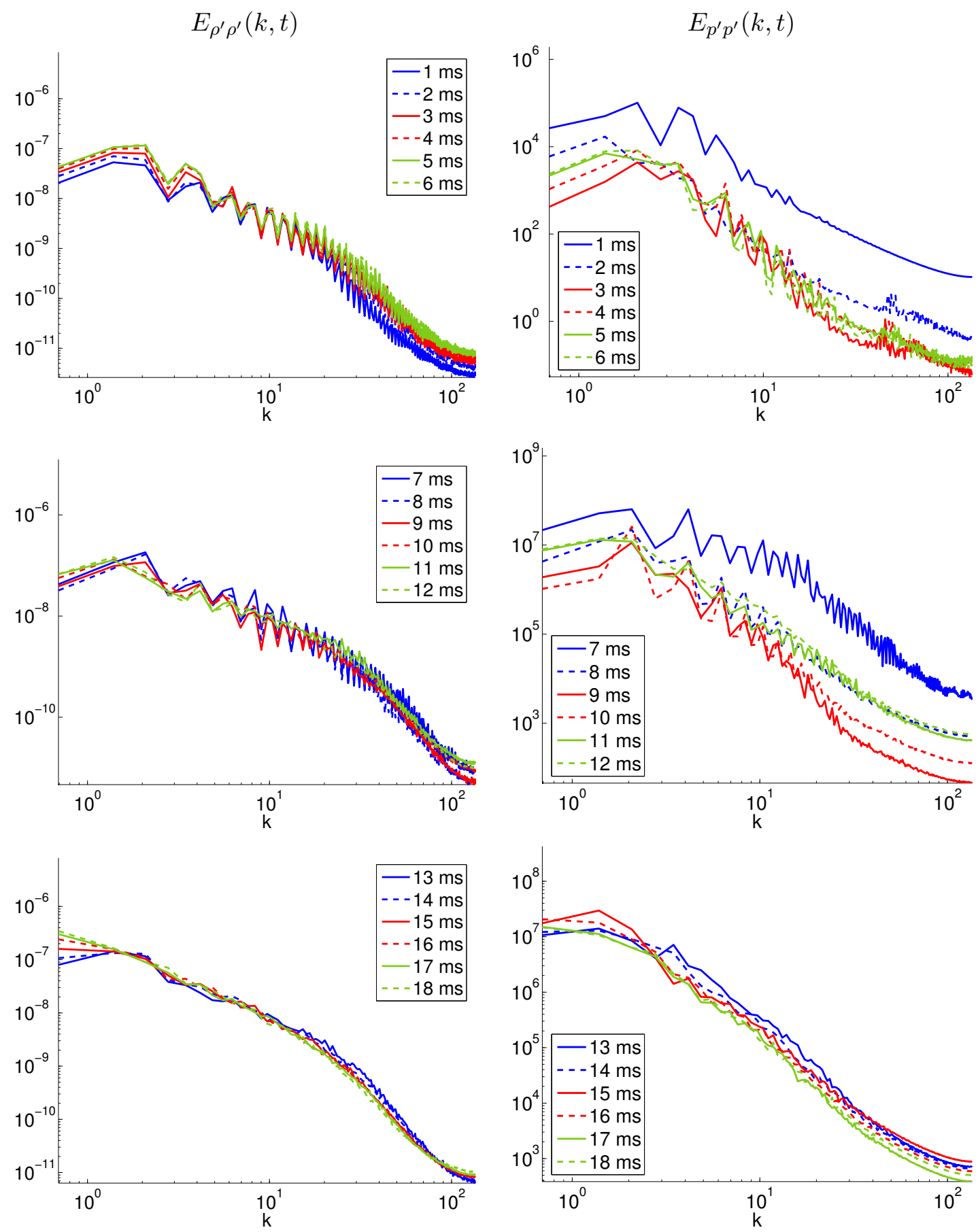

Figure 26: Time-evolution of the density variance spectrum $E_{\rho^{\prime} \rho^{\prime}}(k, t)$ (left column) and the pressure variance spectrum $E_{p^{\prime} p^{\prime}}(k, t)$ (right column). 
streamwise kinetic energy dominate, and this ratio sharply increases. The ratio again decreases at late times, as dissipative mechanisms damp the larger streamwise fluctuations.

Figure 26 shows the time-evolution of the density variance spectrum per unit volume $E_{\rho^{\prime} \rho^{\prime}}(k, t)$ and the pressure variance spectrum per unit volume $E_{p^{\prime} p^{\prime}}(k, t)$. The density variance spectrum shows very little variation with time, indicating that density fluctuations are not as strongly affected by reshock as other quantities. Moreover, the magnitude of the density variance spectrum is very small for all times, indicating that the density variance is very small and that the flow is, therefore, nearly-incompressible. Additional numerical evidence exists to support this conclusion in Richtmyer-Meshkov unstable flows [40]. The issue of nearincompressibility after reshock will be revisited in Part 4 of this report [59]. The evolution of the spectrum also shows the interaction of reflected waves with the mixing layer, which have a profound effect on the pressure variance spectrum. The pressure variance spectrum exhibits a jump as a result of reshock at $t=7 \mathrm{~ms}$, and also exhibits a jump at $t=11 \mathrm{~ms}$ when the reflected rarefaction wave interacts with the mixing layer. At late times, both the pressure and density variance spectra exhibit a slow decay in time. There is no apparent power-law scaling of these spectra.

\subsection{Time-evolution of statistics}

Statistics are obtained by summing the energy over all modes,

$$
\begin{aligned}
E_{\phi \phi}(t) & =\frac{\overline{\phi^{2}}}{2} \\
& =\int_{0}^{\infty} E_{\phi \phi}(k, t) \mathrm{d} k .
\end{aligned}
$$

Numerically, this integral is a sum over all wavenumbers up to the Nyquist wavenumber (127) $k_{\max }$. As a numerical check, statistics were computed by summing the energy spectra over all wavenumbers, $\sum_{n=1}^{N_{y}} E_{\phi \phi}\left(k_{n}, t\right)$, and directly by a volume integration $\sum_{i \in S} \sum_{j=1}^{N_{y}} \phi\left(x_{i}, y_{j}, t\right)^{2} / 2$ where $S=\left\{i: x_{i} \in\left[a_{s}(t), a_{b}(t)\right]\right\}$ to ensure their accurate computation.

Figure 27 shows the time-evolution of the streamwise and spanwise fluctuating kinetic energy per unit volume

$$
\begin{aligned}
E_{u^{\prime \prime} u^{\prime \prime}}(t) & =\frac{\overline{u^{\prime \prime 2}}}{2} \\
& =\int_{0}^{\infty} E_{u^{\prime \prime} u^{\prime \prime}}(k, t) \mathrm{d} k
\end{aligned}
$$

and

$$
\begin{aligned}
E_{v^{\prime \prime} v^{\prime \prime}}(t) & =\frac{\overline{v^{\prime \prime 2}}}{2} \\
& =\int_{0}^{\infty} E_{v^{\prime \prime} v^{\prime \prime}}(k, t) \mathrm{d} k,
\end{aligned}
$$

and the total fluctuating kinetic energy per unit volume

$$
E(t)=E_{u^{\prime \prime} u^{\prime \prime}}(t)+E_{v^{\prime \prime} v^{\prime \prime}}(t)
$$

illustrating the relative magnitude of the two components. Initially, $E_{v^{\prime \prime} v^{\prime \prime}}(t)$ is much smaller than $E_{u^{\prime \prime} u^{\prime \prime}}(t)$ due to the fact that the initial shock primarily excites modes in the streamwise 

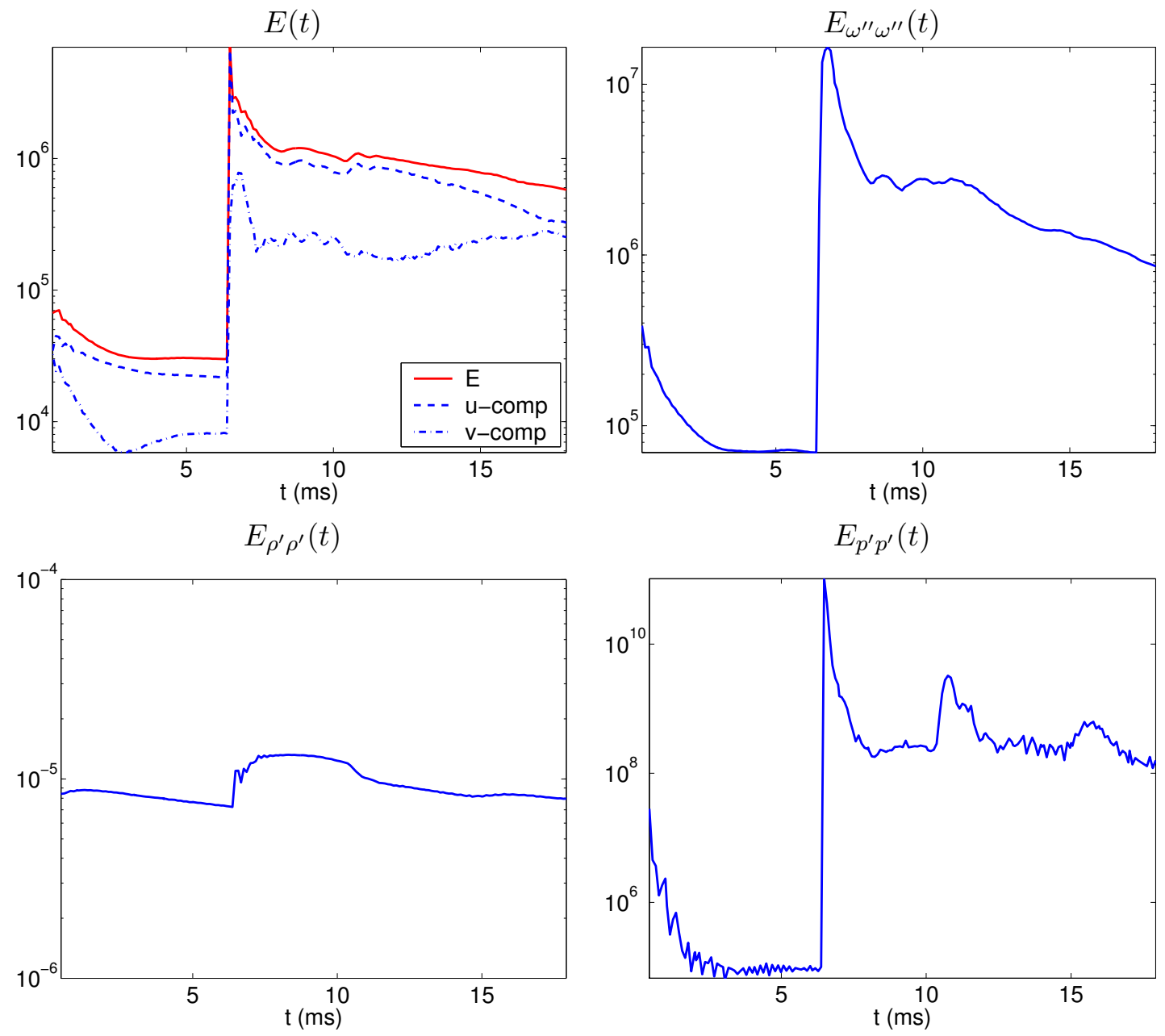

Figure 27: Time-evolution of the fluctuating kinetic energy $E(t)$ and its components, the enstrophy $E_{\omega^{\prime \prime} \omega^{\prime \prime}}(t)$, density variance $E_{\rho^{\prime} \rho^{\prime}}(t)$, and pressure variance $E_{p^{\prime} p^{\prime}}(t)$. 


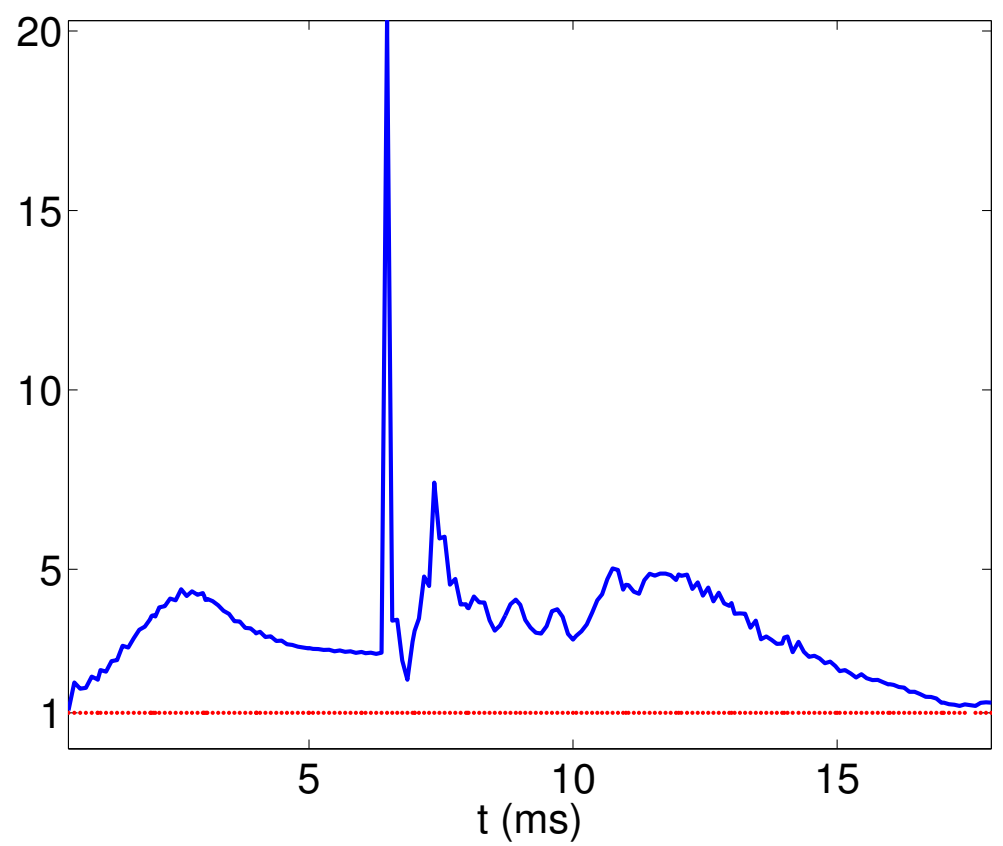

Figure 28: Time-evolution of the ratio of the streamwise and spanwise fluctuating kinetic energy $E_{u^{\prime \prime} u^{\prime \prime}}(t) / E_{v^{\prime \prime} v^{\prime \prime}}(t)$. The ratio approaches unity at late-time, indicating an approach to statistical isotropy. Note, however, that the kinetic energy spectra are still spectrallyanisotropic at late times. 
direction (as shown in Fig. 23). The kinetic energy decreases following the initial shock, while reshock deposits additional vorticity and amplifies the kinetic energy. The reshock and the subsequent reflected waves also excite spanwise velocity fluctuations. The contributions from the spanwise and streamwise fluctuations are very similar at late times, indicating an approach of the flow to statistical (but not spectral) isotropy. The ratio of the spanwise and streamwise kinetic energy is shown in Fig. 28. The ratio approaches unity at late time, indicating an approach to isotropy.

The time-evolution of the fluctuating enstrophy per unit volume

$$
\begin{aligned}
E_{\omega^{\prime \prime} \omega^{\prime \prime}}(t) & =\frac{\overline{\omega^{\prime \prime 2}}}{2} \\
& =\int_{0}^{\infty} E_{\omega^{\prime \prime} \omega^{\prime \prime}}(k, t) \mathrm{d} k
\end{aligned}
$$

is also shown in Fig. 27. Note the large increase in the fluctuating enstrophy during reshock. Following reshock, the enstrophy decays, but the arrival of the reflected waves compensates for the decrease and a nearly constant value is observed. Finally, after the interaction of the reflected rarefaction with the mixing layer at $t=11 \mathrm{~ms}$, the enstrophy decays.

Finally, consider the time-evolution of the density variance

$$
\begin{aligned}
E_{\rho^{\prime} \rho^{\prime}}(t) & =\frac{\overline{\rho^{\prime 2}}}{2} \\
& =\int_{0}^{\infty} E_{\rho^{\prime} \rho^{\prime}}(k, t) \mathrm{d} k
\end{aligned}
$$

and the pressure variance

$$
\begin{aligned}
E_{p^{\prime} p^{\prime}}(t) & =\frac{\overline{p^{\prime 2}}}{2} \\
& =\int_{0}^{\infty} E_{p^{\prime} p^{\prime}}(k, t) \mathrm{d} k .
\end{aligned}
$$

The density variance is not as significantly affected by reshock and by other reflected waves as the other quantities, and remains nearly constant in time. By contrast, the pressure variance is much more sensitive, exhibiting peaks as waves interact with the mixing layer. With the exception of $E_{\rho^{\prime} \rho^{\prime}}(t)$, all of the remaining statistics decrease from their initial values until reshock. Also, with the exception of $E_{\rho^{\prime} \rho^{\prime}}(t)$, all of the remaining statistics decrease sharply following reshock. With the present reflecting boundary condition, the simulation does not achieve a purely-decaying state at late times. Later in $\S 4.6$, the reflecting boundary condition is changed to outflow in order to remove the effects of reflected waves following reshock.

\subsection{Comparison of mixing quantities at selected times}

In the previous sections, a temporal progression of each quantity was presented at time intervals of $1 \mathrm{~ms}$. Here, comparisons of quantities at selected times are presented together to further elucidate the structure of the mixing as characterized by each quantity. The times are selected to reflect key aspects of the flow evolution:

1. at $t=6 \mathrm{~ms}$ after the initial interaction with the shock but before reshock; 
2. at $t=7 \mathrm{~ms}$ immediately after reshock;

3. at $t=12 \mathrm{~ms}$ for large times after reshock, and;

4. at $t=18 \mathrm{~ms}$ for late times.

Also presented is a comparison of quantities just before and after reshock to quantitatively investigate the effect of reshock on the mixing process. These quantities include molar profiles, volume fraction profiles, mixing fractions, and fluctuating energy spectra. Note that in the figures, quantities are shown on an $x$-axis recentered according to the location of the centerline of the mixing layer.

\subsubsection{Comparison of mixing quantities at early and late times}

Figure 29 shows the mixing profiles and spectra at $t=6 \mathrm{~ms}$ in the left column and at $t=7 \mathrm{~ms}$ in the right column. Consider first the mole fraction profile at $t=6 \mathrm{~ms}$ just before reshock. The mole fraction profile $\langle X\rangle$ shows the distribution of mass, indicating the presence of the well-developed roll-up corresponding to the peak. To its right, the decrease in $\langle X\rangle$ corresponds to the bubble of the lighter air(acetone) rising into the heavier $\mathrm{SF}_{6}$. The plot of $\left\langle X_{p}\right\rangle$ shows localized peaks, corresponding to the center of the roll-ups where mixing is most intense. The value of $\left\langle X_{p}\right\rangle$ decreases at the boundaries of the roll-up region. The profile of $X\left(\left\langle X_{p}\right\rangle\right)$ shows a first peak corresponding to the entire roll-up region and a second peak corresponding to the tip of the bubble. Finally, the mixing fraction $\xi$ shows a central peak indicates that mixing is most intense at the center of the roll-ups. Additional peaks are observed at the tip of the spike and at the tip of the bubble, resulting from the rapid decrease in those regions of $X\left(\left\langle X_{p}\right\rangle\right)$. The volume fraction profiles $\left\langle f_{1}\right\rangle$ and $\left\langle f_{2}\right\rangle$ show the relative volumetric distribution of the two fluids across the mixing layer. The molecular mixing fraction $\theta$ shows that the two fluids are mixed the most in the roll-up region. Finally, the fluctuating kinetic energy spectrum $E(k, t)$ shows that most of the energy is contributed by the streamwise velocity component.

Consider the mole fraction profile at $t=7 \mathrm{~ms}$ shortly after reshock. The mass is more evenly distributed, as measured by the monotonic profile of $\langle X\rangle$. Note that the peak previously observed at $t=6 \mathrm{~ms}$ has disappeared as a result of the mass redistribution. Mixing is most intense closer to the pure air(acetone), as measured by $\left\langle X_{p}\right\rangle$ and $\xi$. The decrease in $X_{p}(\langle X\rangle)$ close to the air(acetone) mixture indicates the presence of large, low-density regions. The volume fraction profile $\left\langle f_{1}\right\rangle$ shows qualitatively similar behavior.

Figure 30 shows the mixing profiles and spectra at $t=12 \mathrm{~ms}$ in the left column and at $t=18 \mathrm{~ms}$ in the right column. Consider the mole fraction profile $\langle X\rangle$ at $t=12 \mathrm{~ms}$, which shows a steadily increasing distribution of mass across the layer. This mass is well-mixed in the layer, as indicated by $\left\langle X_{p}\right\rangle$. However, large structures are present near the center of the layer, as indicated by the large values of $X_{p}(\langle X\rangle)$. The plot of $\xi$ indicates a well-mixed region closer to the air(acetone). However, large, low-density regions are present as $X_{p}(\langle X\rangle)$ is very small, due to the very small amount of $\mathrm{SF}_{6}$, as indicated by $\langle X\rangle$. Instead, a region with more mixing is observed closer to the center of the mixing layer. The quantities at $t=18 \mathrm{~ms}$ show increased homogeneous mixing as indicated by: (1) a monotonic distribution of mass captured by $\langle X\rangle$, and the volume fraction profiles $\left\langle f_{1}\right\rangle$ and $\left\langle f_{2}\right\rangle ;(2)$ by a distribution of $\left\langle X_{p}\right\rangle$ that does not exhibit large peaks, and; (3) by a value of $X_{p}(\langle X\rangle)$ exhibiting a nearly constant mean value. Further note that the fluctuating kinetic energy spectrum exhibits little change from

$t=12 \mathrm{~ms}$ to $t=18 \mathrm{~ms}$. The streamwise component continues to dominate the spanwise 

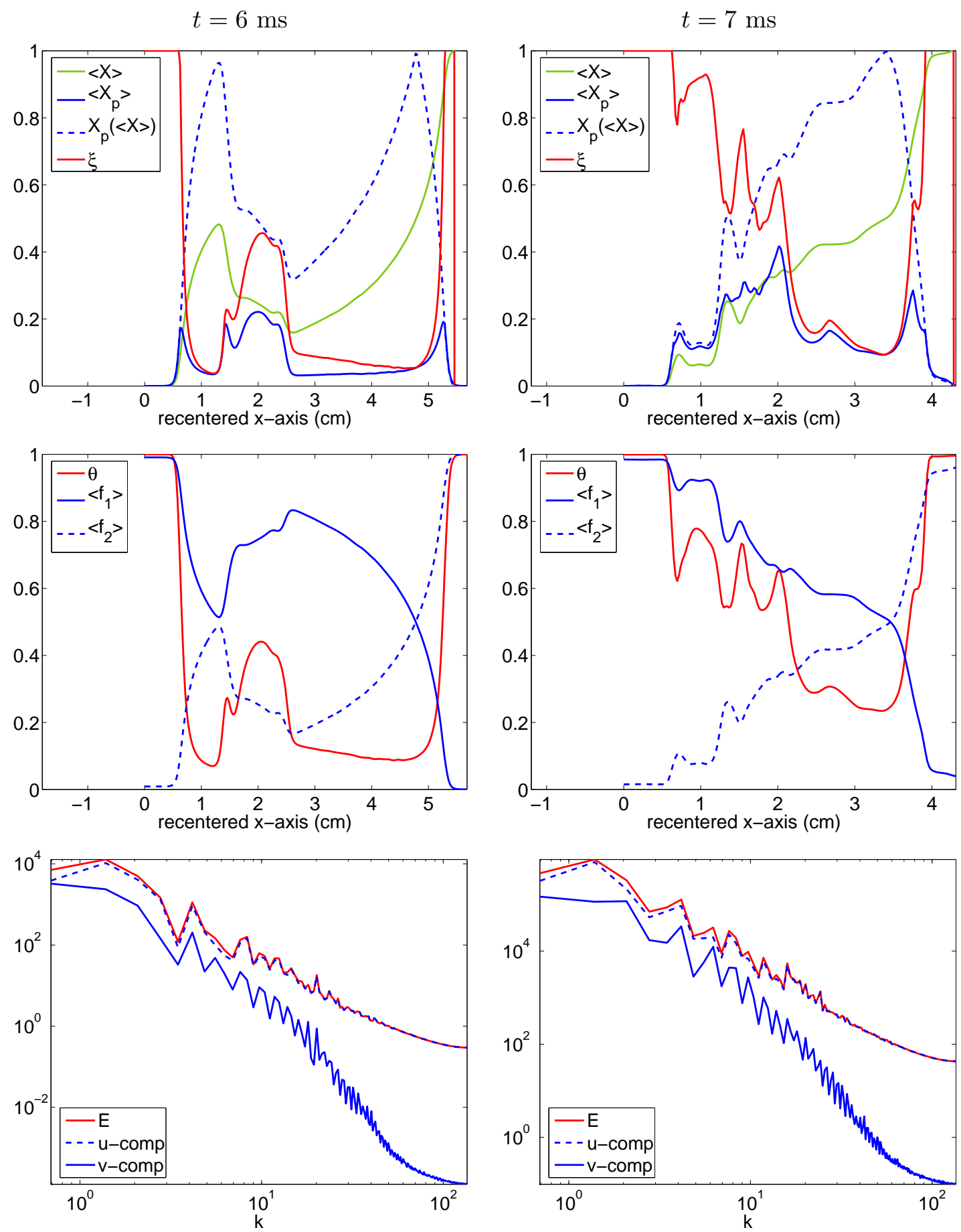

Figure 29: Profiles of $\langle X\rangle,\left\langle X_{p}\right\rangle, X_{p}(\langle X\rangle), \xi,\left\langle f_{1}\right\rangle,\left\langle f_{2}\right\rangle, \theta$, and the fluctuating kinetic energy spectrum $E(k, t)$ and its components at $t=6 \mathrm{~ms}$ (left column) and at $t=7 \mathrm{~ms}$ (right column). 

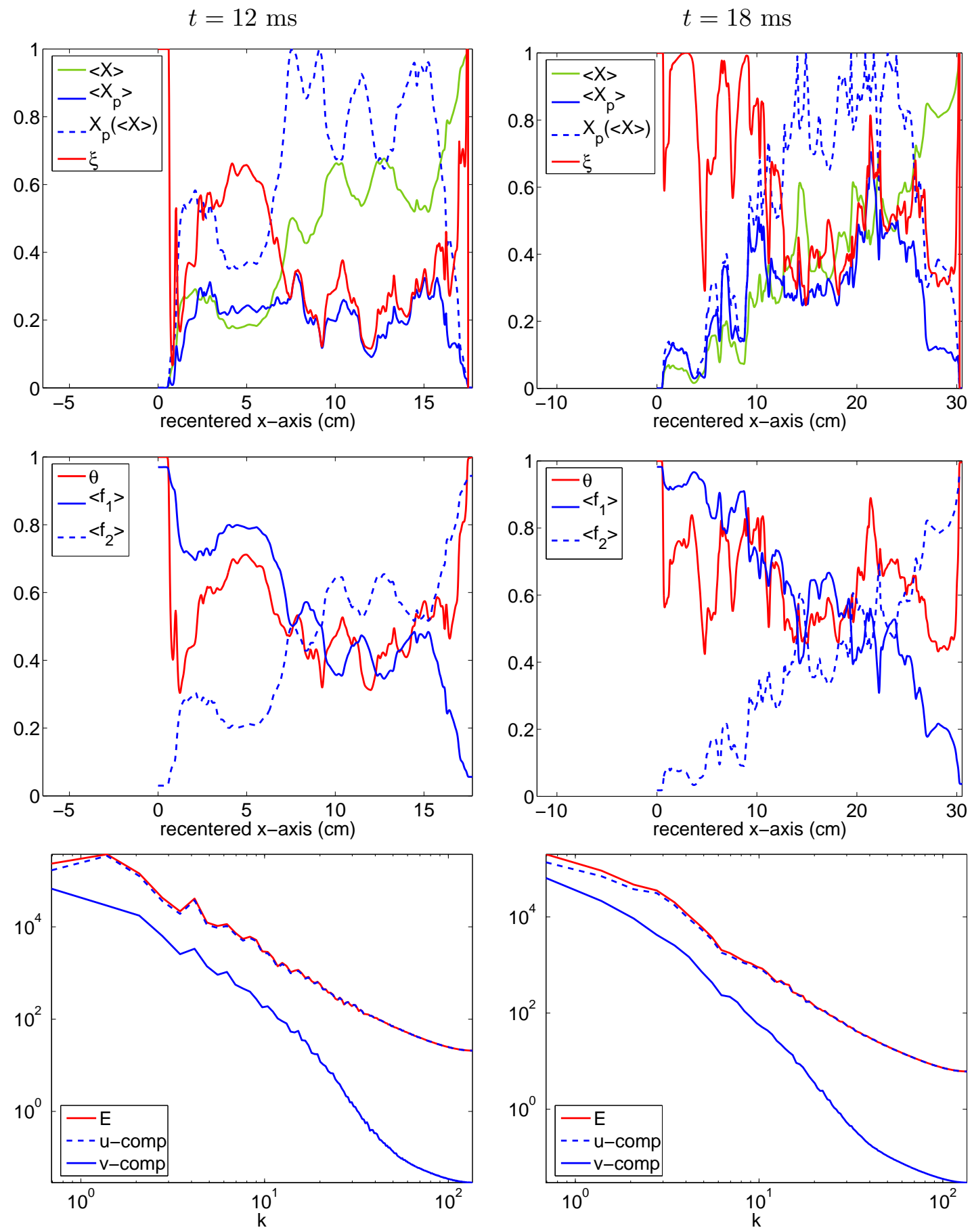

Figure 30: Profiles of $\langle X\rangle,\left\langle X_{p}\right\rangle, X_{p}(\langle X\rangle), \xi,\left\langle f_{1}\right\rangle,\left\langle f_{2}\right\rangle, \theta$, and the fluctuating kinetic energy spectrum $E(k, t)$ and its components at $t=12 \mathrm{~ms}$ (left column) and at $t=18 \mathrm{~ms}$ (right column). 
component by approximately an order of magnitude in the large and intermediate scales, and by approximately three orders of magnitude in the smallest scales.

\subsubsection{Comparison of mixing quantities before and after reshock}

Comparisons of quantities at $t=t^{-}=6.38 \mathrm{~ms}$ immediately prior to reshock and at $t=t^{+}=$ 6.68 immediately following reshock are presented here. The mole fraction profiles are shown in Fig. 31. The $x$-axis has been recentered and rescaled to provide an immediate comparison of the quantities. The following convention is adopted: quantities before and after reshock are shown in blue and red, respectively. The mole fraction profile $\langle X\rangle$ exhibits a complex structure resulting from the compression during reshock. The production quantity $\left\langle X_{p}\right\rangle$ increases during reshock, resulting in greater overall mixing. The largest peak in $\left\langle X_{p}\right\rangle$ located near the pure $\mathrm{SF}_{6}$ is a consequence of the inversion, which flattens the tip of the bubble and also creates small-scale structures. The volume fractions, as well as the mixing fractions $\theta$ and $\xi$, are shown in Fig. 32. The volume fraction profiles $\left\langle f_{1}\right\rangle$ and $\left\langle f_{2}\right\rangle$ show a sharp change close to the pure $\mathrm{SF}_{6}$ region resulting from the flattening of the bubble front. Note the strongly increased mixing, as measured by the mixing fractions $\theta$ and $\xi$ following reshock.

The fluctuating kinetic energy spectrum shown in Fig. 33 sharply increases following reshock. The fluctuating enstrophy, and pressure variance spectra also show sharp increases following reshock. By contrast, the density variance spectrum increases only slightly following reshock.

Finally, Fig. 34 shows the ratio of spectral quantities after and before reshock to quantify the amplification in energy as a function of the wavenumber $k$. The fluctuating kinetic energy spectrum $E(k, t)$ is amplified uniformly by a factor of $\approx 200$ for large wavenumbers. The largest amplification occurs at the largest scales. For the remaining spectra, the largest amplification occurs in the intermediate scales. The fluctuating enstrophy spectrum $E_{\omega^{\prime \prime} \omega^{\prime \prime}}(k, t)$ is amplified differently in different wavenumber regions. The amplification is large for small wavenumbers, reaching a peak of $\approx 1300$ near $k=30$. The amplification reduces for large wavenumbers and oscillates about a value of $\approx 100$. The density variance spectrum $E_{\rho^{\prime} \rho^{\prime}}(k, t)$ does not undergo significant amplification compared with the other spectra, and shows an average amplification of $\approx 2.5$. By contrast, the pressure variance spectrum undergoes the most amplification, showing a peak of $\approx 6 \times 10^{6}$ for $k \approx 20-25$ before relaxing to $\approx 10^{6}$ at large wavenumbers.

\subsection{The effects of reflected waves: outflow and reflecting boundary condi- tions}

Following reshock, the mixing layer undergoes further interactions with reflected waves from the end wall of the shock tube test section. The most significant reflected wave is the rarefaction wave formed during reshock. This wave interacts with the mixing layer at $t \approx 10 \mathrm{~ms}$. The interaction with the rarefaction wave causes the formation of a compression wave, which interacts with the mixing layer at $t \approx 15 \mathrm{~ms}$, as seen in $\langle v\rangle_{x y}$ and $\langle p\rangle_{x y}$ in Fig. 22. In this section, the boundary condition at the right end of the computational domain (corresponding to the end wall of the test section) is varied from reflecting to outflow immediately following reshock. This change allows the reflected rarefaction wave created during reshock to exit the computational domain, so that no further interactions of waves with the mixing layer occur. The purpose of this study is to investigate the properties of mixing in the decay regime, as distinguished from those in the quasi-decay regime occurring when reflected waves interact with the evolving interface following reshock. The following convention is adopted in this section. 
$\langle X\rangle$ recentered

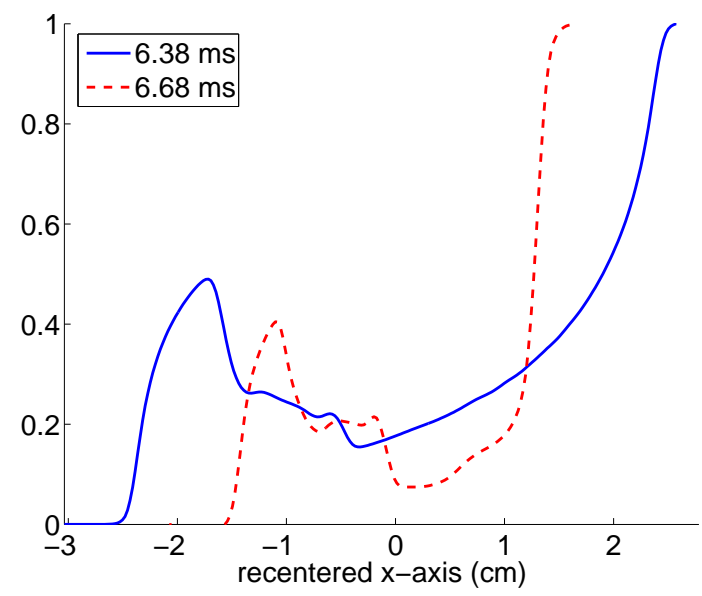

$\left\langle X_{p}\right\rangle$

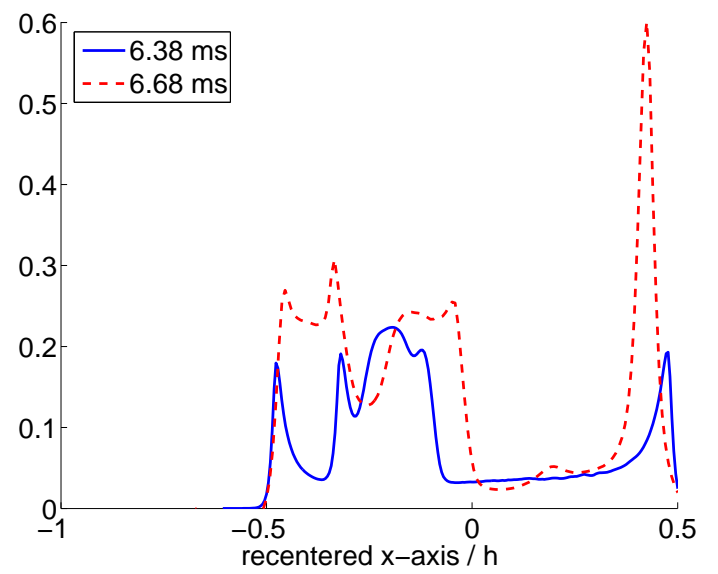

$\langle X\rangle$ recentered and rescaled
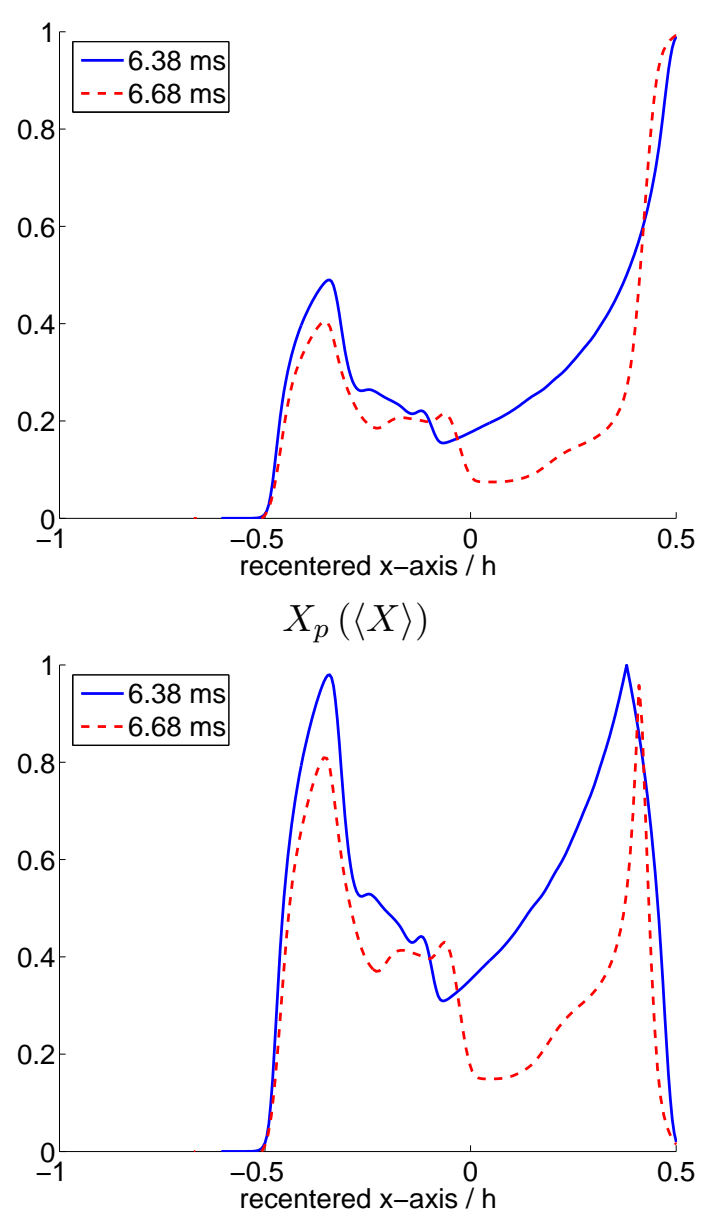

Figure 31: Comparison of $\langle X\rangle,\left\langle X_{p}\right\rangle$, and $X_{p}(\langle X\rangle)$ at $t^{-}=6.38 \mathrm{~ms}$ and at $t^{+}=6.68 \mathrm{~ms}$. 
$\left\langle f_{1}\right\rangle$

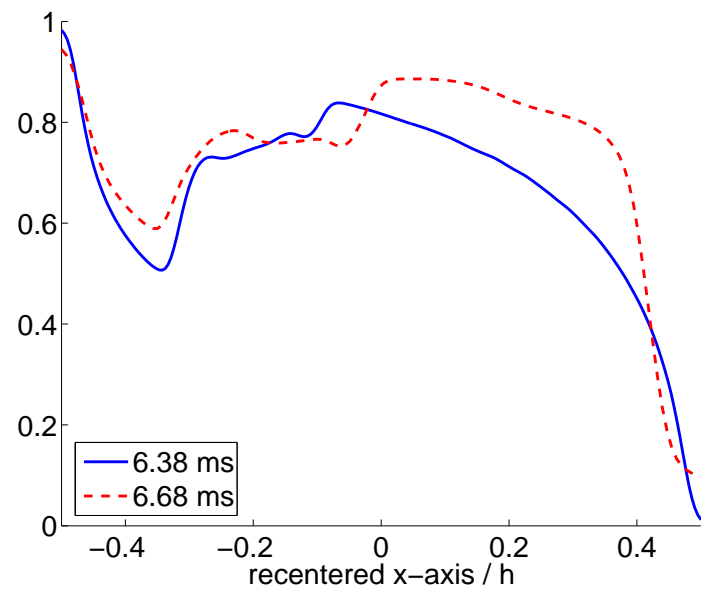

$\theta$

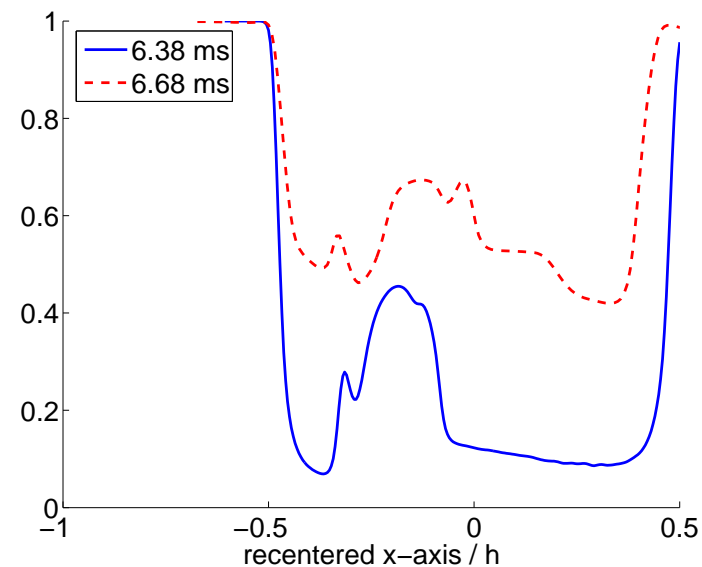

$\left\langle f_{2}\right\rangle$

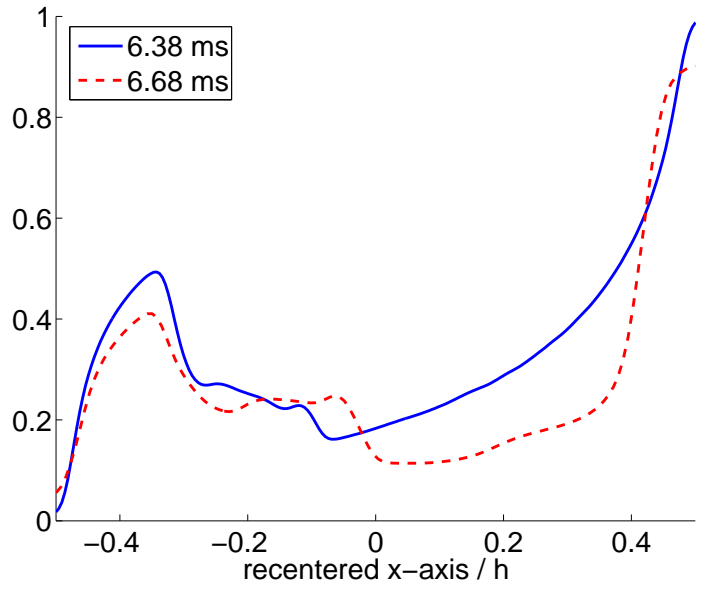

$\xi$

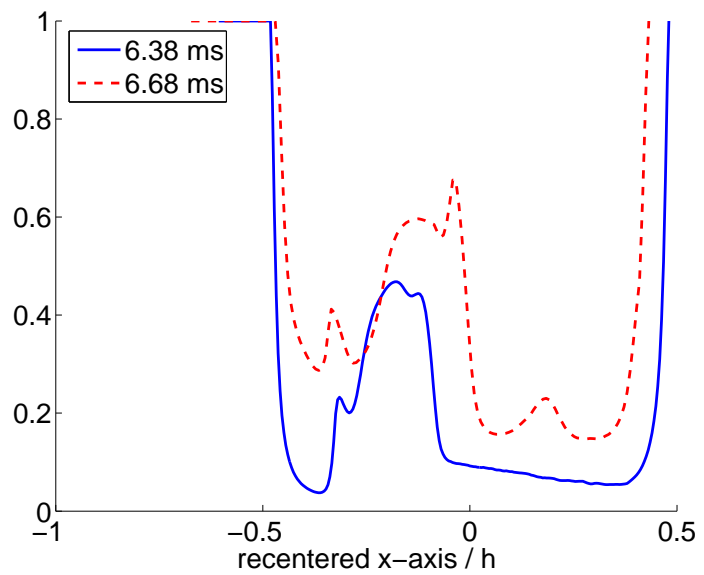

Figure 32: Comparison of $\left\langle f_{1}\right\rangle$ and $\left\langle f_{2}\right\rangle$ and $\theta$ at $t^{-}=6.38 \mathrm{~ms}$ and at $t^{+}=6.68 \mathrm{~ms}$ 

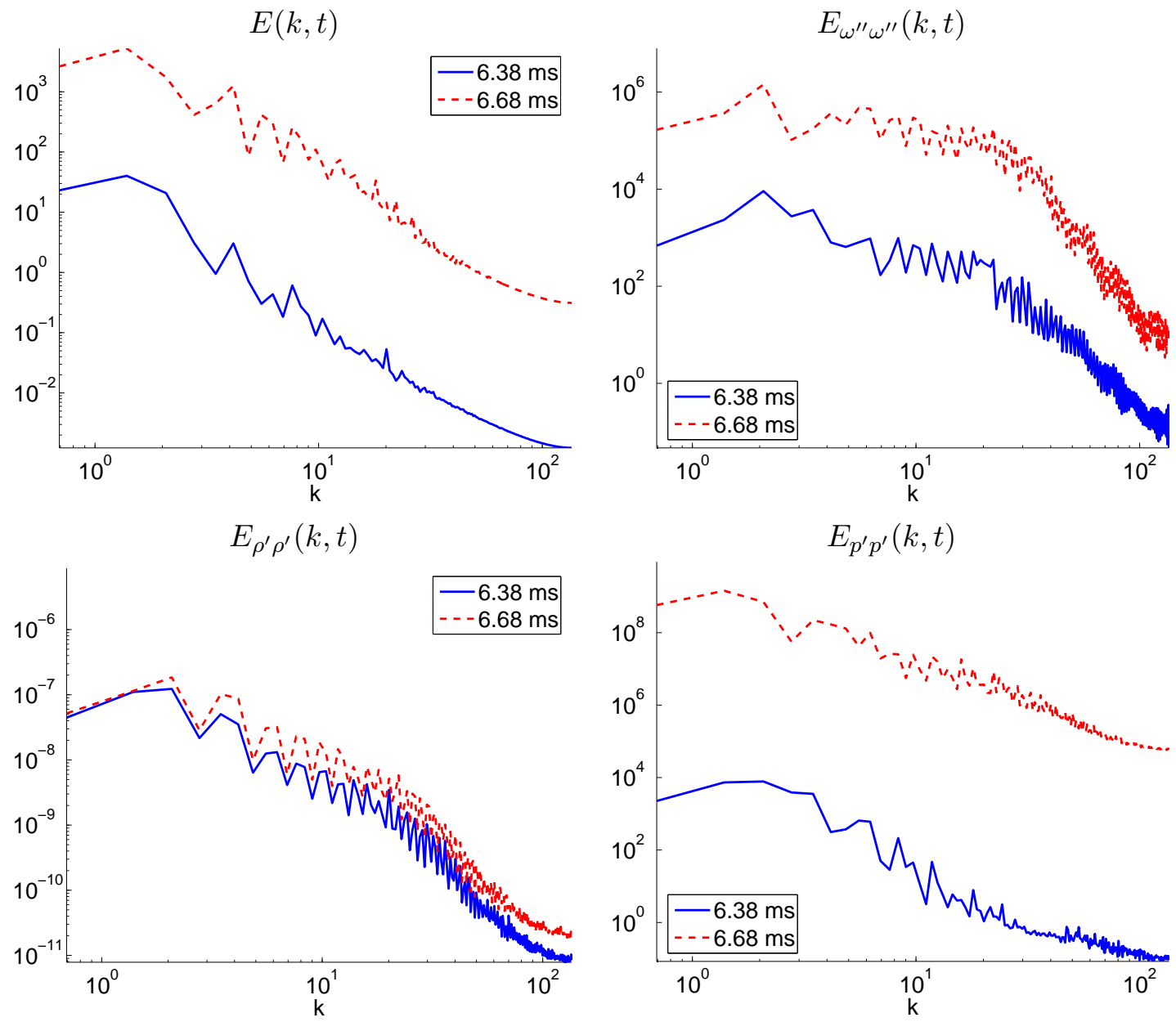

Figure 33: Comparison of the fluctuating kinetic energy spectrum $E(k, t)$, fluctuating enstrophy spectrum $E_{\omega^{\prime \prime} \omega^{\prime \prime}}(k, t)$, density variance spectrum $E_{\rho^{\prime} \rho^{\prime}}(k, t)$, and pressure variance spectrum $E_{p^{\prime} p^{\prime}}(k, t)$ at $t^{-}=6.38 \mathrm{~ms}$ and at $t^{+}=6.68 \mathrm{~ms}$. 

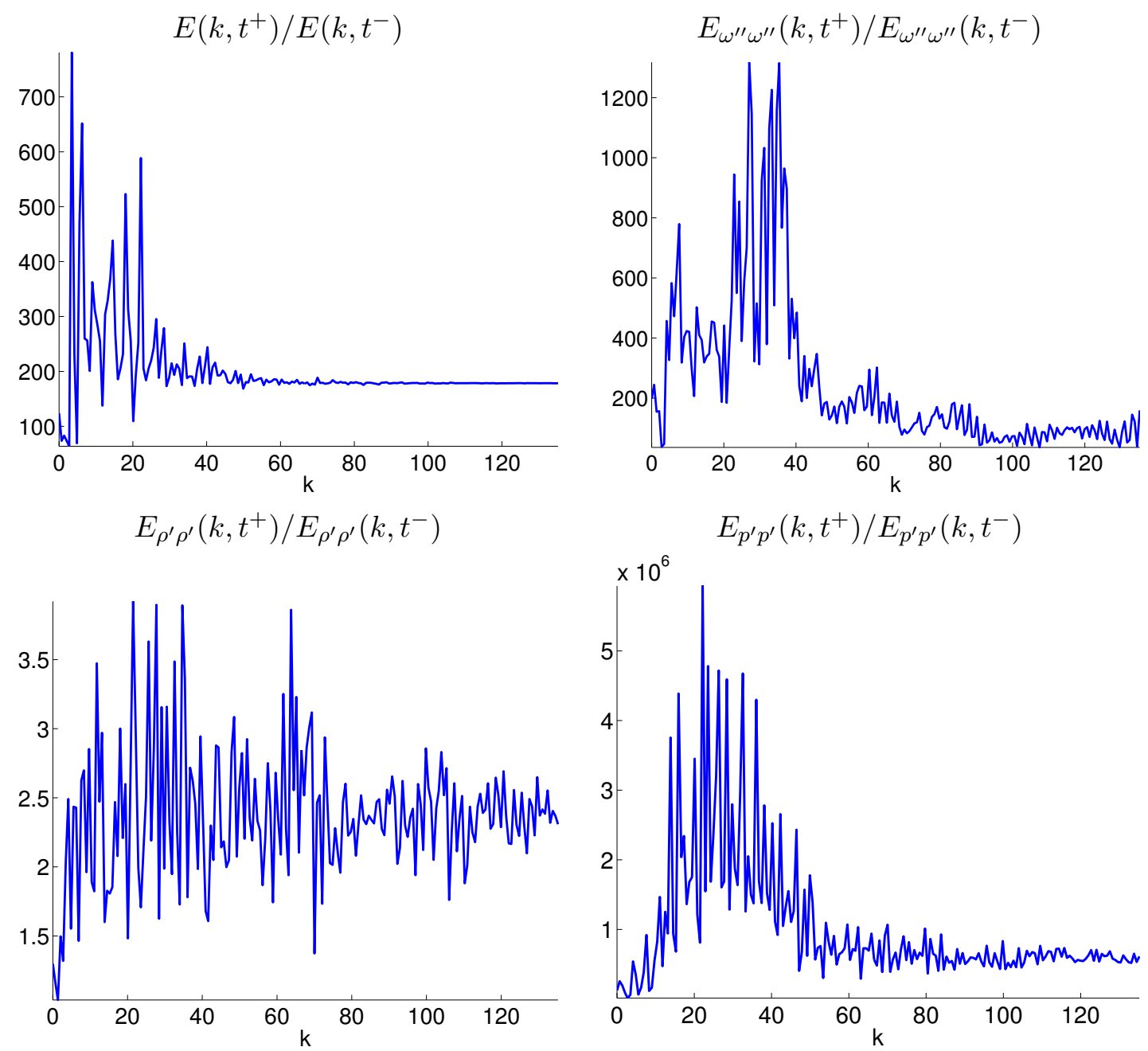

Figure 34: The ratio of fluctuating spectra $E(k, t), E_{\omega^{\prime \prime} \omega^{\prime \prime}}(k, t), E_{\rho^{\prime} \rho^{\prime}}(k, t)$, and $E_{p^{\prime} p^{\prime}}(k, t)$ before reshock at $t^{-}=6.38 \mathrm{~ms}$ and after reshock at $t^{+}=6.68 \mathrm{~ms}$. 
Results obtained when the reflected rarefaction wave exits the computational domain and does not interact with the mixing layer (outflow boundary conditions) are shown in red, and results obtained when the reflected waves interact with the mixing layer (reflecting boundary conditions) are shown in blue. The results overlap from the time of reshock $t=6 \mathrm{~ms}$ until $t \approx 10$ ms when the reflected rarefaction wave interacts with the mixing layer. In general, note that quantities computed with the outflow boundary condition are smoother compared with those computed with the reflecting boundary condition, due to the absence of wave interactions.

\subsubsection{Comparison of mixing layer width, mixing fractions, and profiles}

The top row of Fig. 35 shows the time-evolution of the mixing layer width $h(t)$ and the lengthscale $W(t)$. The reflected rarefaction amplifies the growth rate of the mixing layer. Also shown in the plot of $h(t)$ are the predictions of the Mikaelian and the Brouillette-Sturtevant models for the reshocked interface growth. These quantities were previously shown in Fig. 16 for reflecting boundary conditions. After $t=11 \mathrm{~ms}$, the amplitude corresponding to the outflow boundary condition has a considerably smaller growth rate than that corresponding to the reflecting boundary condition, showing that the reflected rarefaction wave amplifies the growth of the mixing layer. The growth of $W(t)$ qualitatively resembles that of $h(t)$.

The production and mixing fractions $P_{t}, P_{m}, \Xi$, and $\Theta$ are shown in the middle row of Fig. 35. Consider the time-evolution of $P_{t}$ and $P_{m}$. The simulation with the outflow boundary condition yields a slightly larger value of $P_{t}$ than the reflecting boundary condition simulation. The rarefaction wave increases the mixing layer width and therefore, the overall production fraction decreases when averaged over a larger mixing layer. Similarly, $P_{m}$ corresponding to the outflow boundary condition is smaller than that corresponding to the reflecting boundary condition, as the amount of product is decreased. The additional reflected waves induce oscillations in $P_{t}$ and $P_{m}$.

Consider the the time-evolution of the mixing fractions $\Xi=P_{t} / P_{m}$ and $\Theta$. For the outflow boundary condition, $\Xi$ and $\Theta$ are larger than for the reflecting boundary condition, as the reflected rarefaction wave increases the mixing layer width and, thus, decreases the overall mixing as measured by these fractions.

The time-evolution of the volume-averaged streamwise and spanwise velocities $\langle u\rangle_{x y}$ and $\langle v\rangle_{x y}$, and pressure $\langle p\rangle_{x y}$ are shown in the bottom row of Fig. 35. As expected, $\langle u\rangle_{x y}$ does not approach zero at late times in the case of the outflow boundary condition, but remains at a nearly constant value following reshock. In both cases, $\langle v\rangle_{x y}=0$. The volume-averaged pressure $\langle p\rangle_{x y}$ also remains at a nearly constant value following reshock.

\subsubsection{Comparison of statistics}

A comparison of the evolution of statistics for reflecting and outflow boundary conditions is shown in Fig. 36. Consider the fluctuating kinetic energy per unit mass $E(t)$ and its components $E_{u^{\prime \prime} u^{\prime \prime}}(t)$ and $E_{v^{\prime \prime} v^{\prime \prime}}(t)$. In the case of the outflow boundary condition, the energy is not increased by the reflected waves, and therefore decays. The reflected rarefaction wave also increases the energy in the spanwise component, contributing to the statistical isotropization of the flow. This is not observed in the case of the outflow boundary condition, in which both components retain their separation.

Consider the evolution of the fluctuating enstrophy $E_{\omega^{\prime \prime} \omega^{\prime \prime}}(t)$. The enstrophy for the case of reflecting and outflow boundary conditions are very similar, indicating that the reflected waves do not increase the enstrophy significantly. 


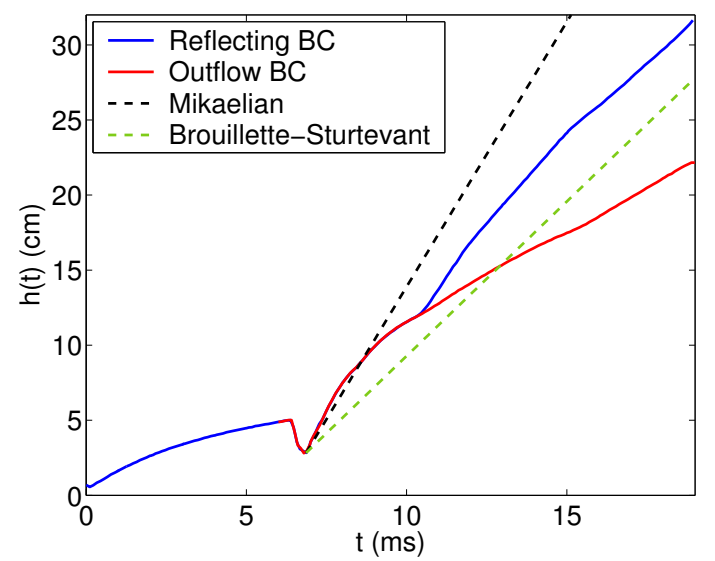

$P_{t}$ and $P_{m}$

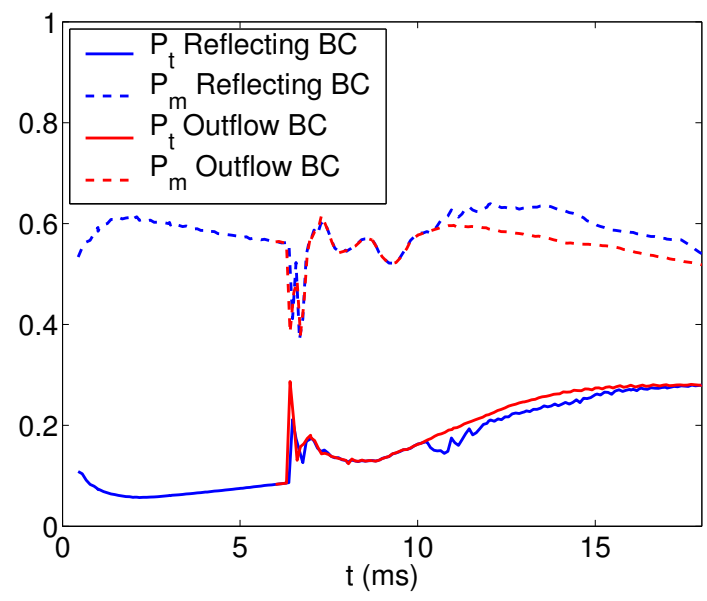

$\langle u\rangle_{x y}$ and $\langle v\rangle_{x y}$

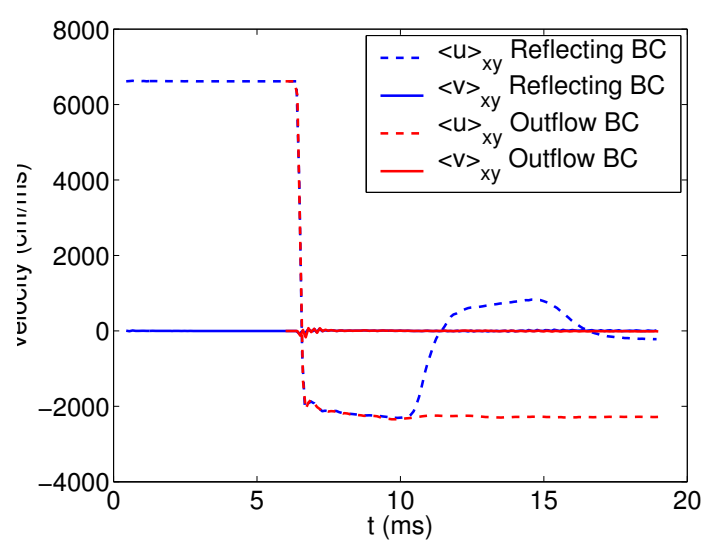

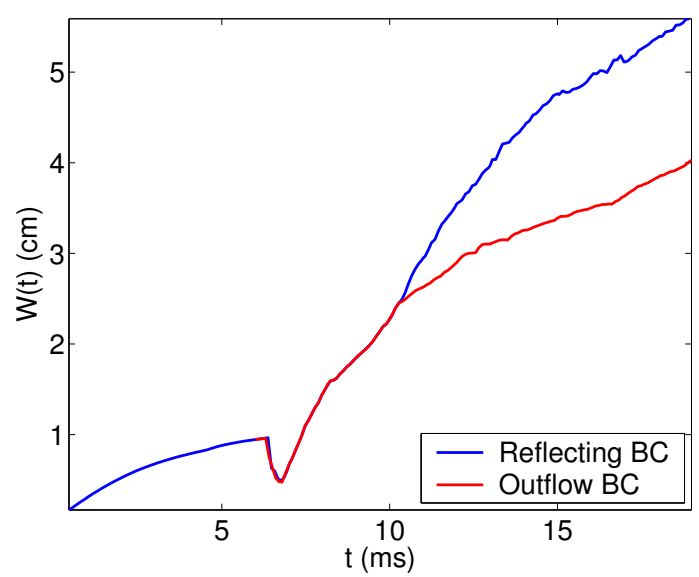

$\Theta$ and $\Xi$

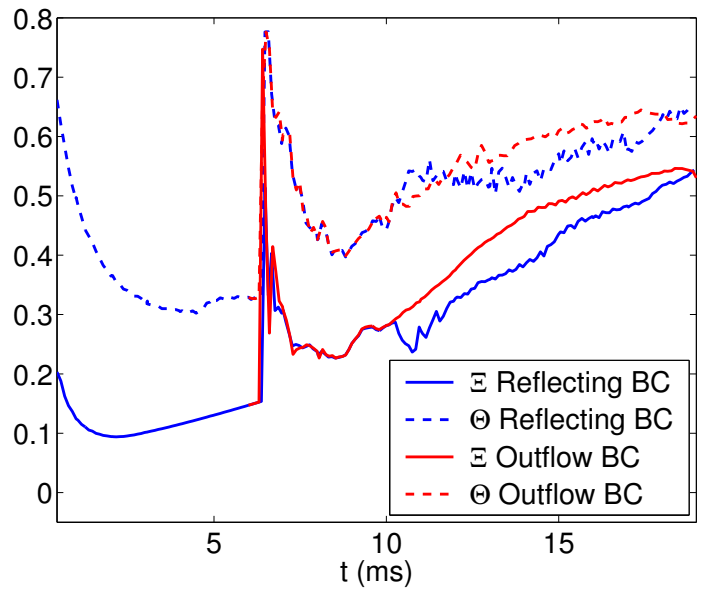

$\langle p\rangle_{x y}$

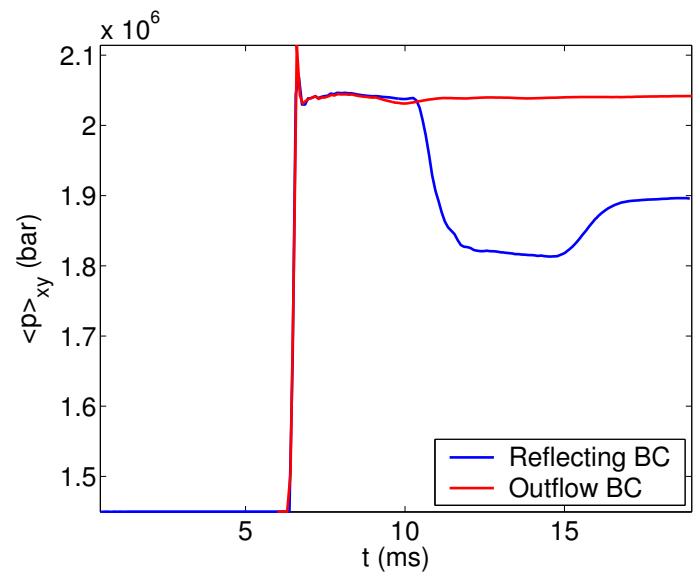

Figure 35: Time-evolution of the mixing layer widths $h(t)$ and $W(t)$, the production fractions $P_{t}$ and $P_{m}$, mixing fractions $\theta$ and $\xi$, and volume-averaged velocities $\langle u\rangle_{x y},\langle v\rangle_{x y}$ and pressure $\langle p\rangle_{x y}$ for reflecting and outflow boundary conditions. 
The behavior of the density variance $E_{\rho^{\prime} \rho^{\prime}}(t)$ is unusual. Note that $E_{\rho^{\prime} \rho^{\prime}}(t)$ corresponding to the outflow boundary condition is greater than $E_{\rho^{\prime} \rho^{\prime}}(t)$ corresponding to the reflecting boundary condition, indicating that the reflected rarefaction wave decreases the density fluctuations.

The pressure variance $E_{p^{\prime} p^{\prime}}(t)$ indicates that the pressure fluctuations are nearly constant in the absence of reflected waves. By contrast, the fluctuations corresponding to the reflecting boundary condition undergo a sudden transient increase following the interaction with reflected waves, and approach the nearly constant value corresponding to the outflow boundary condition shortly thereafter. 

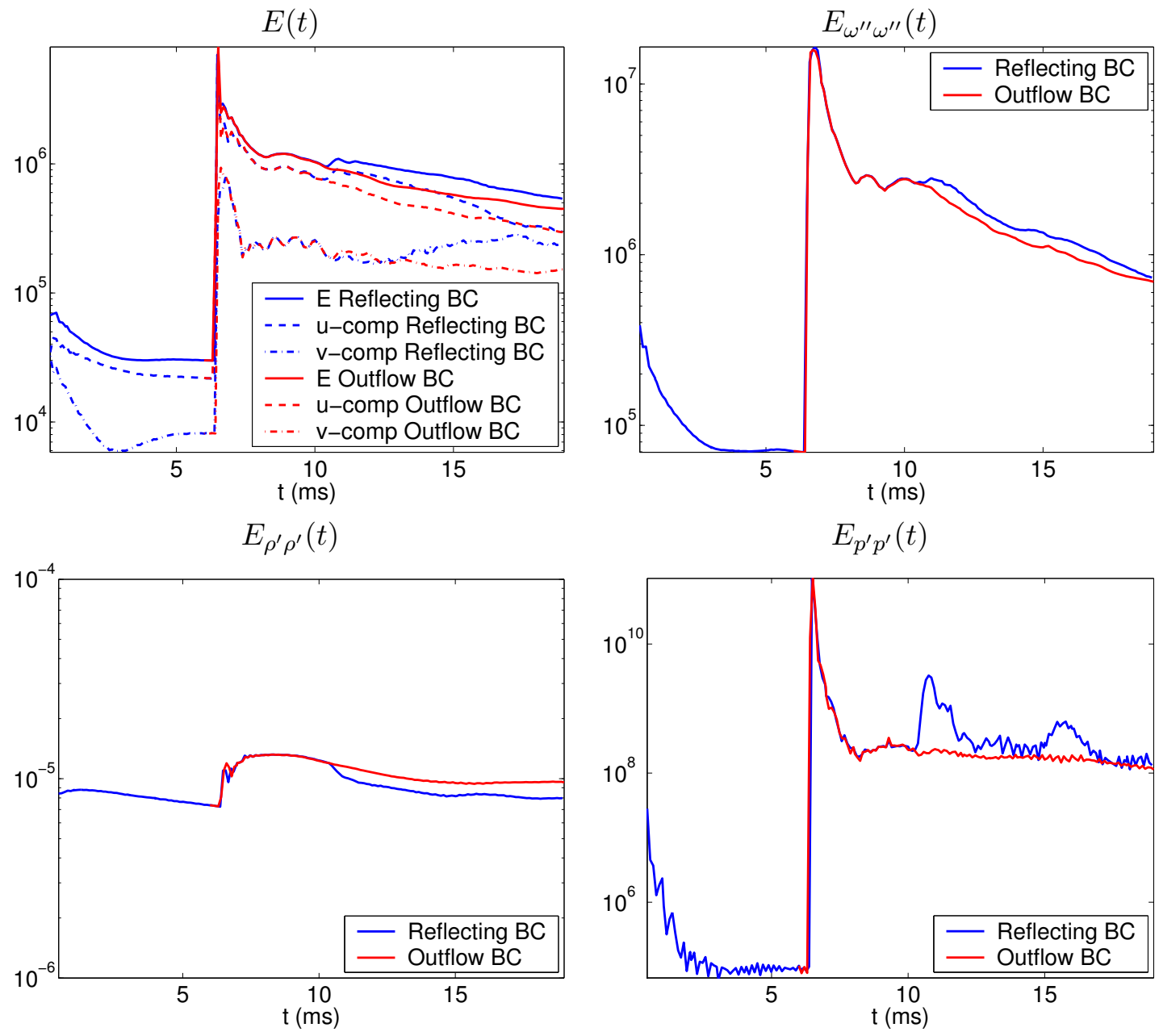

Figure 36: Time-evolution of the fluctuating kinetic energy $E(t)$ and its components, the fluctuating enstrophy $E_{\omega^{\prime \prime} \omega^{\prime \prime}}(t)$, density variance $E_{\rho^{\prime} \rho^{\prime}}(t)$, and pressure variance $E_{p^{\prime} p^{\prime}}(t)$ for outflow and reflecting boundary conditions. 


\section{The effects of spatial resolution and order of spatial recon- struction on mixing}

The effects of the spatial (grid) resolution and order of spatial reconstruction on the mixing quantities are self-consistently and quantitatively investigated in this section. Third-, fifth-, and ninth-order reconstructions are considered, together with grid resolutions of 128, 256, and 512 points per initial perturbation wavelength $\lambda$. The objective of this study is to determine the sensitivity of mixing quantities to the order of reconstruction (formal, or design, order of accuracy in sufficiently smooth flow regions) and to the spatial resolution. Most simulation studies involving the solution of the Euler equations have focused on the numerical 'convergence' of some small set of quantities (or a quantity) (e.g., perturbation amplitude or mixing layer width in Rayleigh-Taylor [97, 37, 26] and Richtmyer-Meshkov instability [42, 41, 22]) over a limited range of time with respect to increasing spatial resolution. The comparison of quantities obtained using different orders of reconstruction and grid resolution in the present work is conducted in the spirit of the investigation of Shi, Zhang and Shu [93], who considered two-dimensional double Mach reflection and Rayleigh-Taylor instability using the fifth- and ninth-order WENO method. Their investigation emphasized the computational advantage of higher-order WENO schemes over lower-order WENO schemes for complex flows mainly using qualitative (visual) comparisons. The present investigation is both quantitative and qualitative, and examines to what extent different orders of WENO reconstruction and different spatial resolutions capture specific physical quantities characterizing Richtmyer-Meshkov instabilityinduced mixing. The investigation is applied to all of the quantities considered in the previous section, including mole fractions, mixing fraction, energy spectra, and statistics. To our knowledge, this is the first systematic investigation of profiles, spectra, and statistics as a function of both spatial resolution and order of reconstruction in the case of the Richtmyer-Meshkov instability. The HOPE code is ideal for such an investigation, as it is possible to perform numerical simulations identical in every other respect except the order of reconstruction: this allows a self-consistent study that is distinct from utilizing different numerical methods with different formal orders of accuracy (see [24] where the results from a high-order Godunov method and a compact finite-difference method applied to the Richtmyer-Meshkov instability are compared).

The following conventions are adopted in the presentation of the results in this section. Quantities obtained using third-, fifth-, and ninth-order WENO reconstruction are shown in green, red, and dark blue, respectively; different line styles are used to present results with varying grid resolution. The simulations obtained with an initial resolution of 512,256 , and 128 points per initial perturbation wavelength are denoted as fine, medium, and coarse grid, respectively, and are presented using a solid line, dashed line, and dash-dot line, respectively. The results presented in $\S 4$ were obtained using the medium resolution grid and fifth-order reconstruction: this choice was based on the fact that the fifth-order WENO method has been used for other investigations by a large number of investigators, while the ninth-order WENO method is relatively new [9].

This section is organized as follows. First, details of the numerical simulations are presented in $\S 5.1$. The results for the mixing layer width are presented in $\S 5.2$, followed by the results for the time-evolution of local mixing quantities and mixing fractions in $\S 5.3$ and $\S 5.4$, respectively. The results for spectra and statistics are presented in $\S 5.5$ and $\S 5.6$, respectively. Finally, the time-evolution of the density and vorticity is shown at intervals of $1 \mathrm{~ms}$ in $\S 5.7$ to provide qualitative comparisons. 


\begin{tabular}{|c|ccc|}
\hline & Fine Grid & Medium Grid & Coarse Grid \\
\hline \hline$N$ per $\lambda$ & 512 & 256 & 128 \\
$\Delta x(\mathrm{~cm})$ & 0.01159 & 0.02318 & 0.04636 \\
$N_{y}$ & 1536 & 768 & 384 \\
$L_{y}(\mathrm{~cm})$ & {$[0,17.8]$} & {$[-0.00579,17.79621]$} & {$[-0.01739,17.78462]$} \\
$N_{x}($ initial $)$ & 801 & 401 & 201 \\
$L_{x}(\mathrm{~cm})($ initial $)$ & {$[0,9.271875]$} & {$[-0.00579,9.26608]$} & {$[-0.01739,9.25449]$} \\
\hline
\end{tabular}

Table 2: The initial grid resolutions used in the numerical simulations. The choice of starting and ending points ensures that all of the grids are aligned with one another.

\subsection{Grid resolutions and orders of reconstruction}

Numerical simulations were performed using uniform grid resolutions with $\Delta x=\Delta y$. The grids were chosen so that points of the medium grid corresponded with every other point of the fine grid. Similarly, the coarse grid was chosen so that its points would correspond to every other point of the medium grid and with every four points of the fine grid.

To define the grids, first chose the grid spacings

$$
\begin{aligned}
\Delta x & =\Delta y \\
& =\frac{\lambda}{N},
\end{aligned}
$$

rounding to a rational number divisible by four, yielding $(\Delta x)_{\text {fine }}=0.01158984375 \mathrm{~cm}$ slightly larger than the theoretical value $\Delta x=\lambda / 512=0.01158854167 \mathrm{~cm}$. This choice corresponds to 768 points in the domain with $L_{y}=8.9 \mathrm{~cm}$. Similarly, $(\Delta x)_{\text {medium }}=0.0231796875 \mathrm{~cm}$ with $N=384$, and $(\Delta x)_{\text {coarse }}=0.046359375 \mathrm{~cm}$ with $N=192$. To align the grids, the starting and ending points of the computational domain were adjusted to account for the distribution of points by the numerical method. In the $y$-direction, the first and second grid points are at locations $y_{1}=-\Delta x / 2$ and $y_{2}=\Delta x / 2$. For this reason, if the starting location is chosen to be at $y=0$ for all grids, then the grids would not be aligned. Thus, choose $y=0$ for the fine grid, corresponding to the first two grid points at $y_{1}=-(\Delta x)_{\text {fine }} / 2$ and $y_{2}=(\Delta x)_{\text {fine }} / 2$. For the medium grid, shift by $(\Delta x)_{\text {fine }} / 2$ to the left so that the position of the first two points are at $y_{1}=-3(\Delta x)_{f i n e} / 2$ and $y_{2}=(\Delta x)_{\text {fine }} / 2$. For the coarse grid, shift by $3(\Delta x)_{\text {fine }} / 2$, yielding $y_{1}=-5(\Delta x)_{\text {fine }} / 2$ and $y_{2}=(\Delta x)_{\text {fine }} / 2$. In all three cases, the points at $y_{2}$ coincide. A similar procedure is followed for the $x$-direction. The actual values for the number of grid points in the $y$-direction and for the length of the domain $L_{y}$ used in the simulations were double the values described above, as the symmetry option in the HOPE code was activated (see [57]). The grid resolutions are summarized in Table 2.

\subsection{The effect on the mixing layer width}

Shown in Fig. 37 is a comparison of the dependence of the mixing layer width on the grid resolution and on the order of the WENO reconstruction up to $t=18 \mathrm{~ms}$. Also shown in Fig. 37 is the ratio of the widths with respect to the width measured for the ninth-order simulation on the fine grid. Prior to reshock, the mixing layer width is weakly-dependent on the resolution and on the order of reconstruction with all simulations giving very similar 
results. This is due to the fact that the flow is dominated by a single, large-scale evolving bubble (or spike), the front of which is not significantly affected by dissipation. However, following reshock, the dependence on both order and resolution becomes significant. In particular, decreasing the order of the WENO reconstruction, as well as decreasing the grid resolution, result in smaller mixing layer widths and vice versa. Note that the differences are further amplified by the arrival of the reflected rarefaction wave at $t \approx 10 \mathrm{~ms}$ and later further increase. These differences can be understood as follows. Lower order of reconstruction and coarser grids correspond to higher values of numerical diffusion. Increased numerical diffusion damps the velocity fluctuations and in particular, the streamwise fluctuations associated with the growth of the mixing layer. Therefore, increased numerical diffusion yields smaller mixing layer widths. In addition, the formation of small-scale structures is also inhibited resulting in smaller baroclinic circulation deposition during a wave/interface interaction compared to a high-resolution, high-order simulation exhibiting small-scale structures. As a result, the mixing layer width is further decreased. This mechanism is clearly seen in $\S 5.7$ where a comparison of the flow features before and after reshock is presented.

Consider the flow evolution following reshock but prior to the arrival of the reflected rarefaction wave in the time interval $t \in[6.5,10.5] \mathrm{ms}$. The mixing layer widths become closer as the grid resolution doubles and the order of reconstruction increases. At late times, the amplitudes from the third-order simulations differ by $\approx 5 \mathrm{~cm}$ as the grid is refined. By contrast the amplitudes obtained from the fifth- and ninth-order simulations differ by $\approx 1 \mathrm{~cm}$ as the grid is refined. The plot of the ratio of the widths show evidence of 'convergence' as the value approaches unity for the ninth order simulations.

\subsection{The effect on mixing profiles}

An analysis of the effects of grid refinement and order of reconstruction on quantities representative of local mixing are presented in this section. The comparisons are conducted at time $t=6 \mathrm{~ms}$ immediately before reshock, at $t=7 \mathrm{~ms}$ immediately after reshock, at $t=12 \mathrm{~ms}$ late after reshock, and at $t=18 \mathrm{~ms}$ at very late time following reshock as in $\S 4.5$.

Figure 38 shows comparisons of the mole fraction profile $\langle X\rangle$ at the selected times. At $t=6 \mathrm{~ms}$, there is generally good agreement once the order and resolution are sufficiently high. The results corresponding to third-order reconstruction at medium resolution are closer to the results corresponding to fifth-order reconstruction at coarse resolution. Similar behavior is observed for times immediately following reshock at $t=7 \mathrm{~ms}$, with results in generally good agreement as the resolution and the order are increased. At $t=12 \mathrm{~ms}$, the results begin to diverge, indicating that differences between the small-scale features are more pronounced and are captured by the mole fraction. This behavior is also observed when considering the late-time behavior at $t=18 \mathrm{~ms}$.

Figure 39 shows comparisons of the product mole fraction profile $\left\langle X_{p}\right\rangle$. This quantity is extremely sensitive to both the order and resolution for all times. Note that the spikes at the beginning and end (corresponding to the top of the bubble and bottom of the spike) are not captured equally between the methods, due to the different amounts of numerical dissipation that result in a larger or smaller well-mixed, diffusion layer. Consider time $t=6 \mathrm{~ms}$ before reshock and note that the larger values closer to the middle of the layer (corresponding to the roll-ups) appear different between the orders and resolutions, with no apparent agreement. This is due to the different structure of the roll-ups observed across the different methods, as shown in $\S 5.7$. As the order is increased and the grid points are doubled, additional spiral 

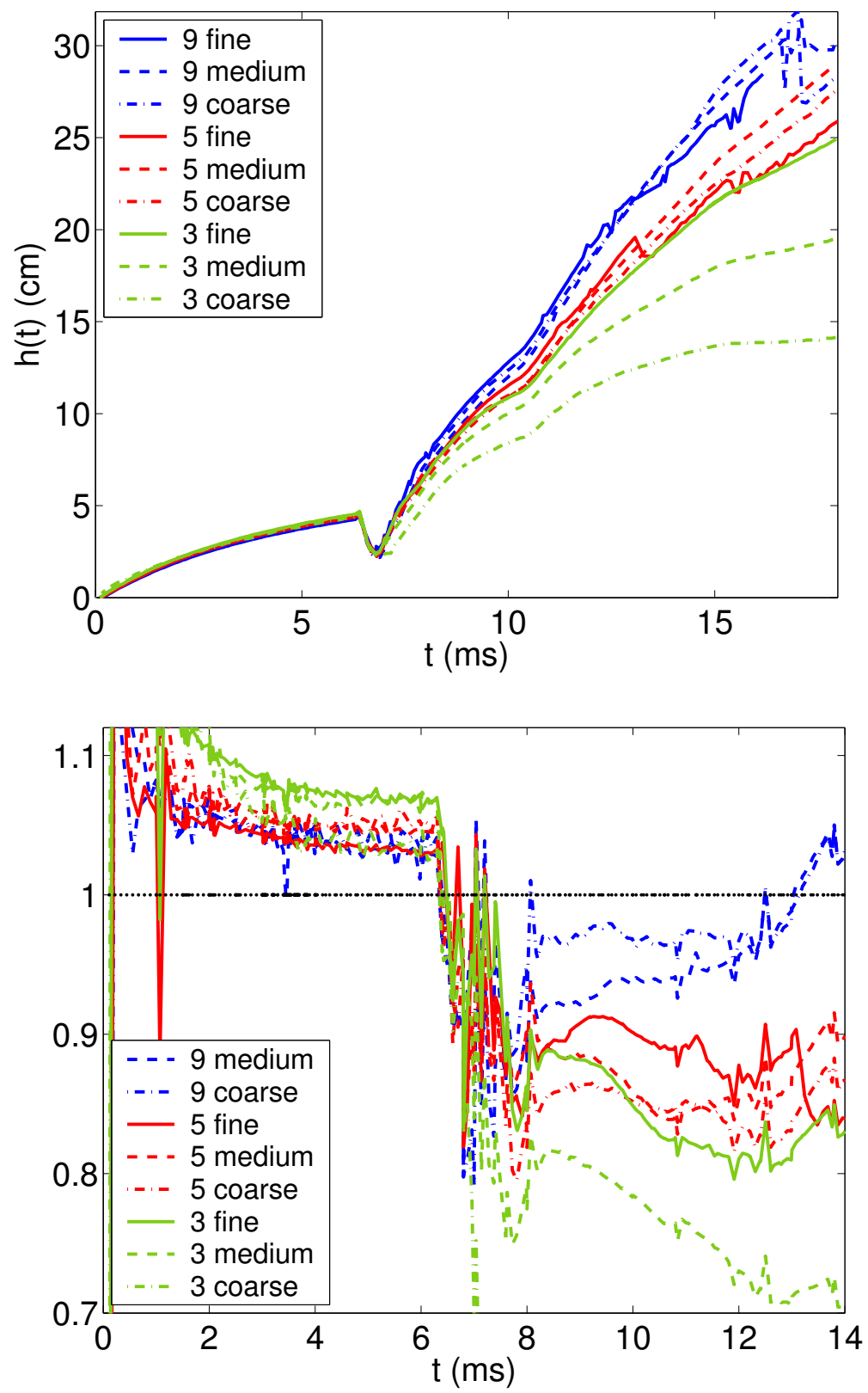

Figure 37: The effects of grid resolution and order of reconstruction on the mixing layer width (top). For times prior to reshock, a weak dependence on the resolution and order is observed. For times following reshock, a stronger dependence is observed, with lower-order reconstruction and coarser grids resulting in smaller post-reshock widths. This is due to the increased numerical dissipation associated with lower-order reconstruction and coarser grids. The ratios of the mixing layer width with respect to the ninth-order simulation on the fine grid (bottom). Following reshock, the ratios corresponding the ninth-order simulations on the medium and coarse grids are bounded between $\approx 0.95-1.05$ for $t>10 \mathrm{~ms}$, providing evidence for 'convergence'. The mixing layer widths obtained from the fifth- and third-order simulations show no 'convergence', even at late times following reshock. 
$t=6 \mathrm{~ms}$
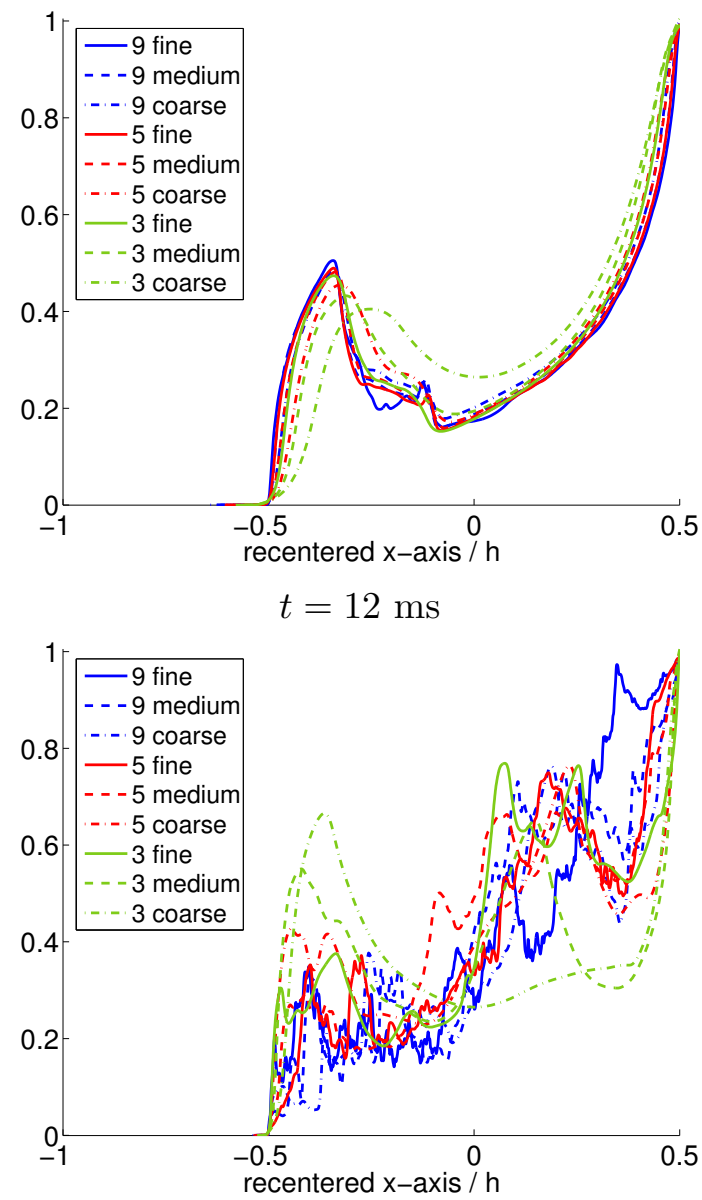

$t=7 \mathrm{~ms}$
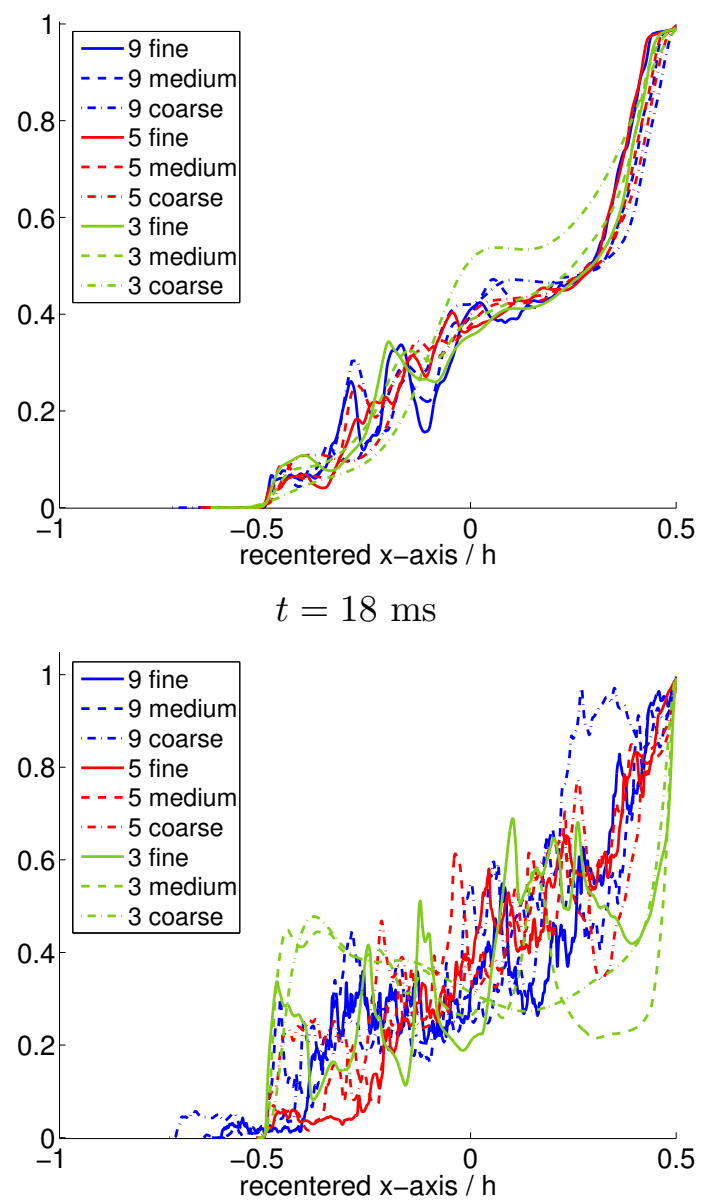

Figure 38: Comparison of the mole fraction profile $\langle X\rangle$ at $t=6,7,12,18$ ms when the order of reconstruction and grid resolution are varied. 
roll-ups appear, causing the product mole fraction to oscillate for high orders of reconstruction. At $t=7 \mathrm{~ms}$, the product mole fraction obtained with increasing order of reconstruction and grid resolution exhibits differences across the entire mixing layer: these differences are further amplified for later times. Note that the results obtained using the third-order WENO method at coarse resolution are in very poor agreement with all of the other results. Again, this is due to the very large numerical dissipation present in low-order WENO reconstructions, and the different structure in the small-scale flow features that is further amplified after interactions with reflected waves.

Figure 40 shows the product mole fraction profile $X_{p}(\langle X\rangle)$. At $t=6 \mathrm{~ms}$ (before reshock), the profiles are in close agreement as the resolution and order of reconstruction are increased. This agreement persists at $t=7 \mathrm{~ms}$ immediately after reshock, but the agreement decreases at later times. The reason for this difference is that $X_{p}(\langle X\rangle)$ is most sensitive to the small-scale flow features. The differences in small-scale features are not captured by $X_{p}(\langle X\rangle)$ until long after reshock. Therefore, as the order is increased and the resolution is doubled, additional small-scale features with a different spatial distribution are observed at late times, causing $X_{p}(\langle X\rangle)$ to exhibit large variations.

Finally, Figs. 41 and 42 show the molecular mixing fractions $\theta$ and $\xi$. The molecular mixing fraction $\theta$ is very sensitive to the order and resolution at all times. Consider $\theta$ at $t=6 \mathrm{~ms}$. No clear agreement is observed, with the smallest and largest values obtained from the ninth- and fifth-order simulations on the fine grid, respectively, indicating that $\theta$ is highly sensitive to the numerical parameters. Note that $\xi$ at $t=6 \mathrm{~ms}$ shows agreement as the order and resolution are increased, indicating that $\xi$ is more robust than $\theta$. Similar observations apply at $t=7 \mathrm{~ms}$. For late times, the mixing fractions are very sensitive to the structure of the flow resulting from the differences in order and grid resolution. The high-amplitude oscillations in $\theta$ and $\xi$ observed at late times indicate that the structures become more fragmented as the resolution and order are increased.

\subsection{The effect on mixing fractions}

The effects of grid refinement and order of reconstruction on the production and mixing fractions are presented in this section.

The top row in Fig. 43 shows the time-evolution of the production fractions $P_{t}(t)$ and $P_{m}(t)$. The same observations noted for $\left\langle X_{p}\right\rangle$ and $X_{p}(\langle X\rangle)$ in Figs. 39 and 40 apply to $P_{t}(t)$ and $P_{m}(t)$. In particular, the large differences observed for $\left\langle X_{p}\right\rangle$ at $t=6 \mathrm{~ms}$, particularly in the regions outside the roll-ups where the difference is due to the width of the diffusion layer, result in increased values of $P_{t}$ as the resolution and order of reconstruction are decreased. Diffusion is also central to understanding the large differences observed after reshock, and to the fact that the results using third-order reconstruction at low resolutions are very different from the other results. The plot of $P_{m}$ shows an interesting trend: before reshock, the values corresponding to third-order reconstruction at low resolution are the lowest, while after reshock the values corresponding to ninth-order reconstruction at high resolution are the lowest. Smaller values of $P_{m}$ indicate that the two fluids are less mixed, as less product is formed. Prior to reshock, the third-order results display roll-ups that are less tight when compared to the fifth- and ninthorder results, resulting in overall decreased product formation. Following reshock, the ninthorder results have the largest mixing layer width, resulting in less overall product formation.

The bottom row of Fig. 43 shows the mixing fractions $\Xi$ and $\Theta$. As noted earlier, $\Xi$ and $\Theta$ quantify (numerical diffusion-induced) mixing. Although both quantities provide similar 

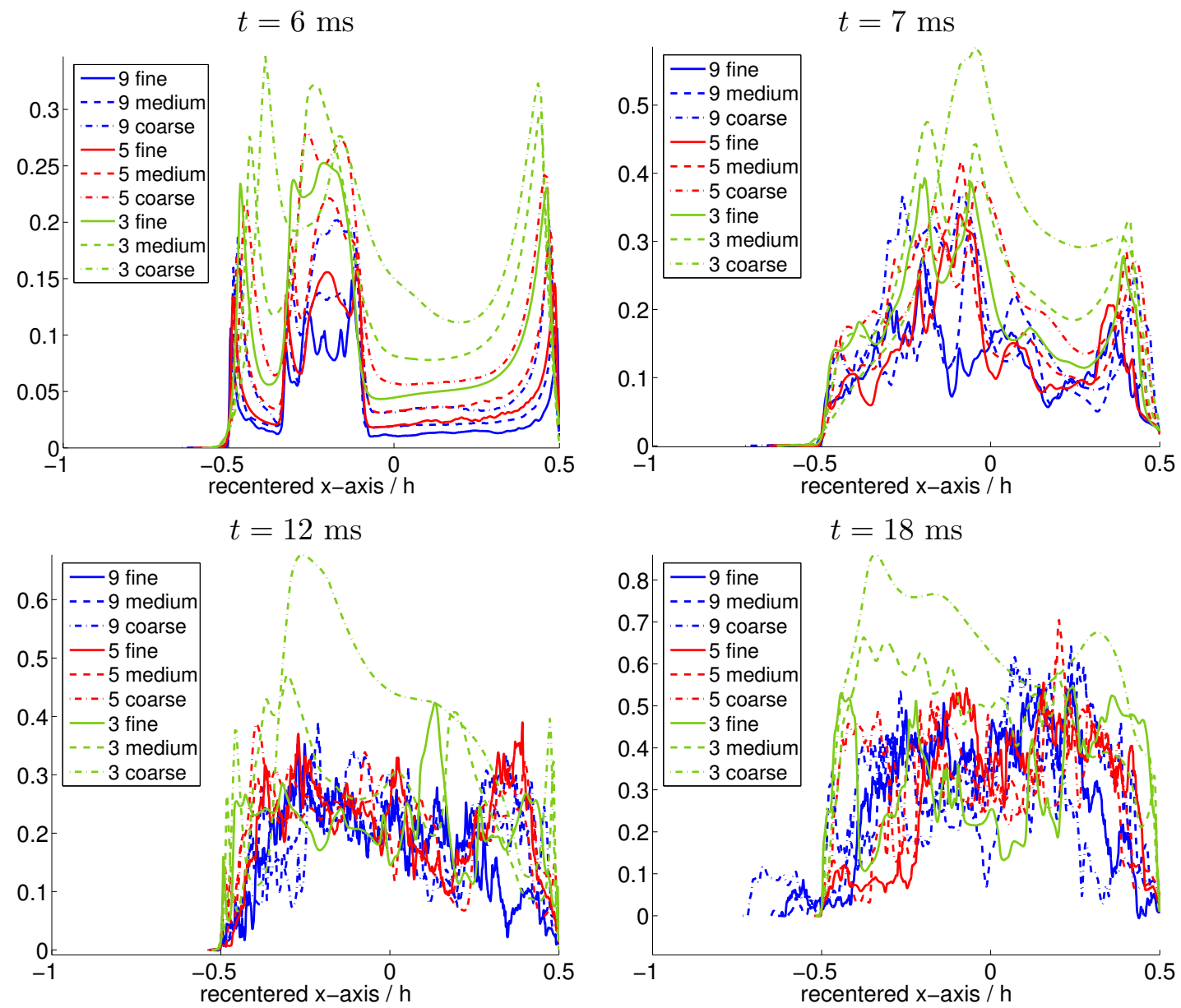

Figure 39: Comparison of the product mole fraction profile $\left\langle X_{p}\right\rangle$ at $t=6,7,12,18 \mathrm{~ms}$ when the order of reconstruction and grid resolution are varied. 

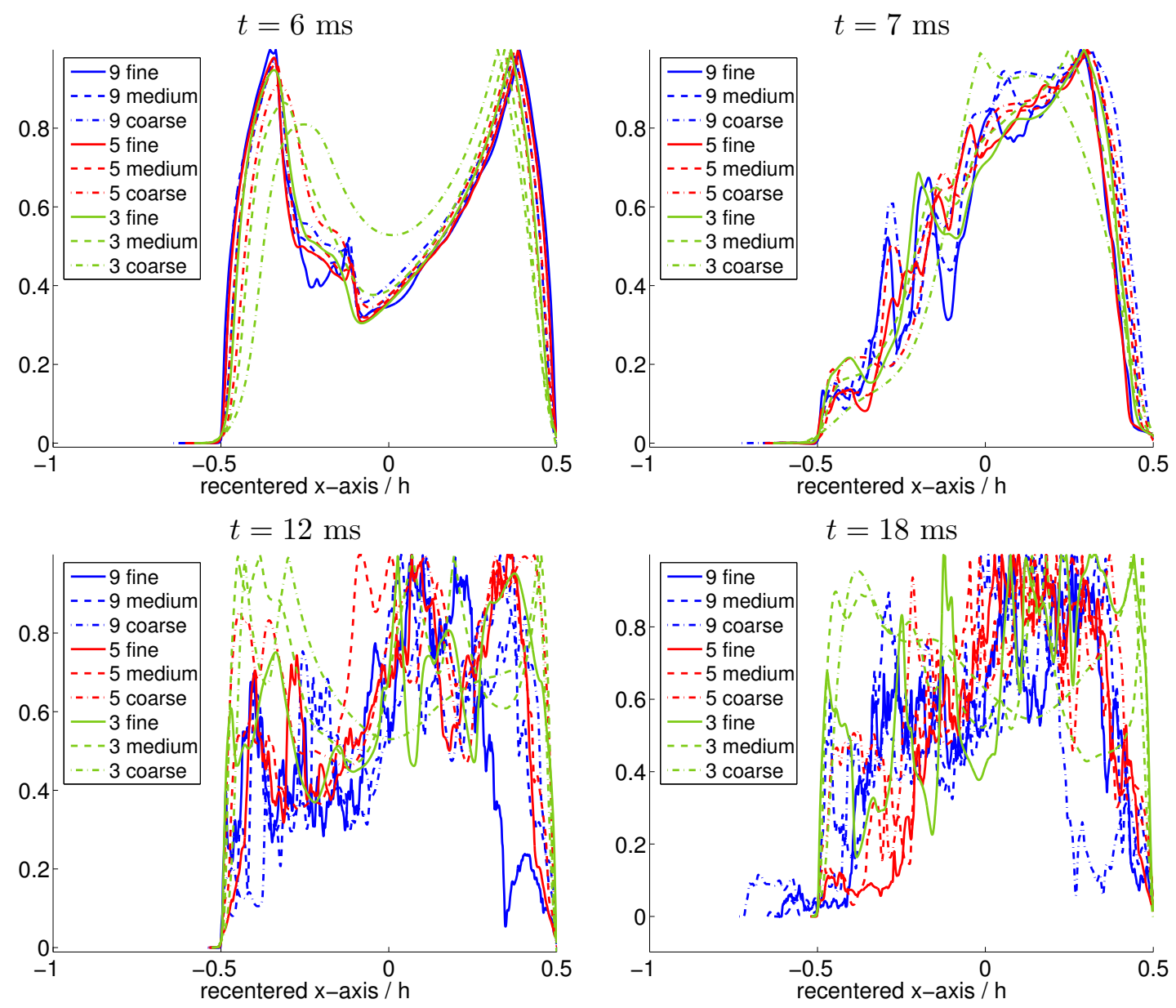

Figure 40: Comparison of the product mole fraction profile $X_{p}(\langle X\rangle)$ at $t=6,7,12,18 \mathrm{~ms}$ when the order of reconstruction and grid resolution are varied. 

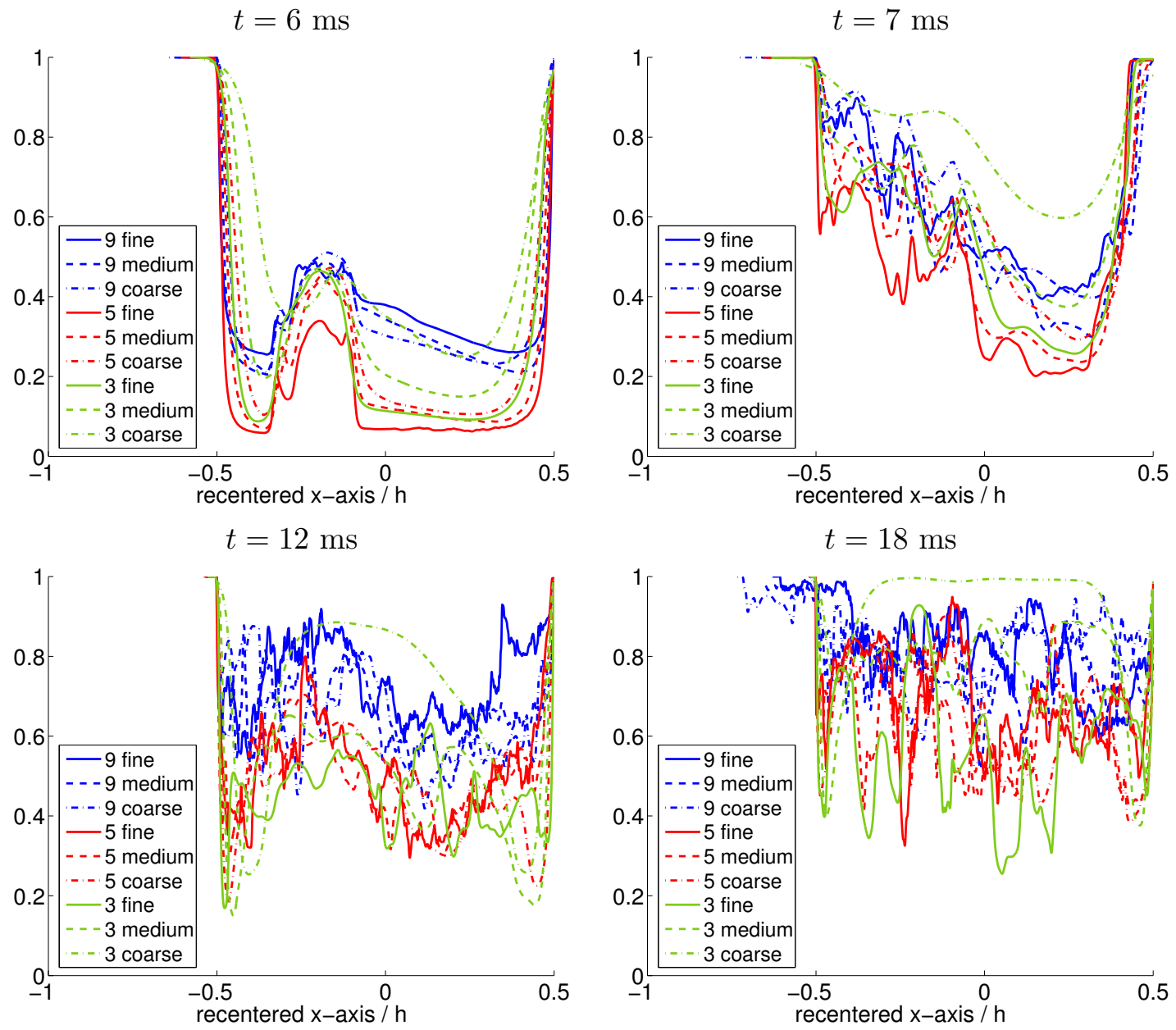

Figure 41: Comparison of the molecular mixing fraction $\theta$ at $t=6,7,12,18$ ms when the order of reconstruction and grid resolution are varied. 

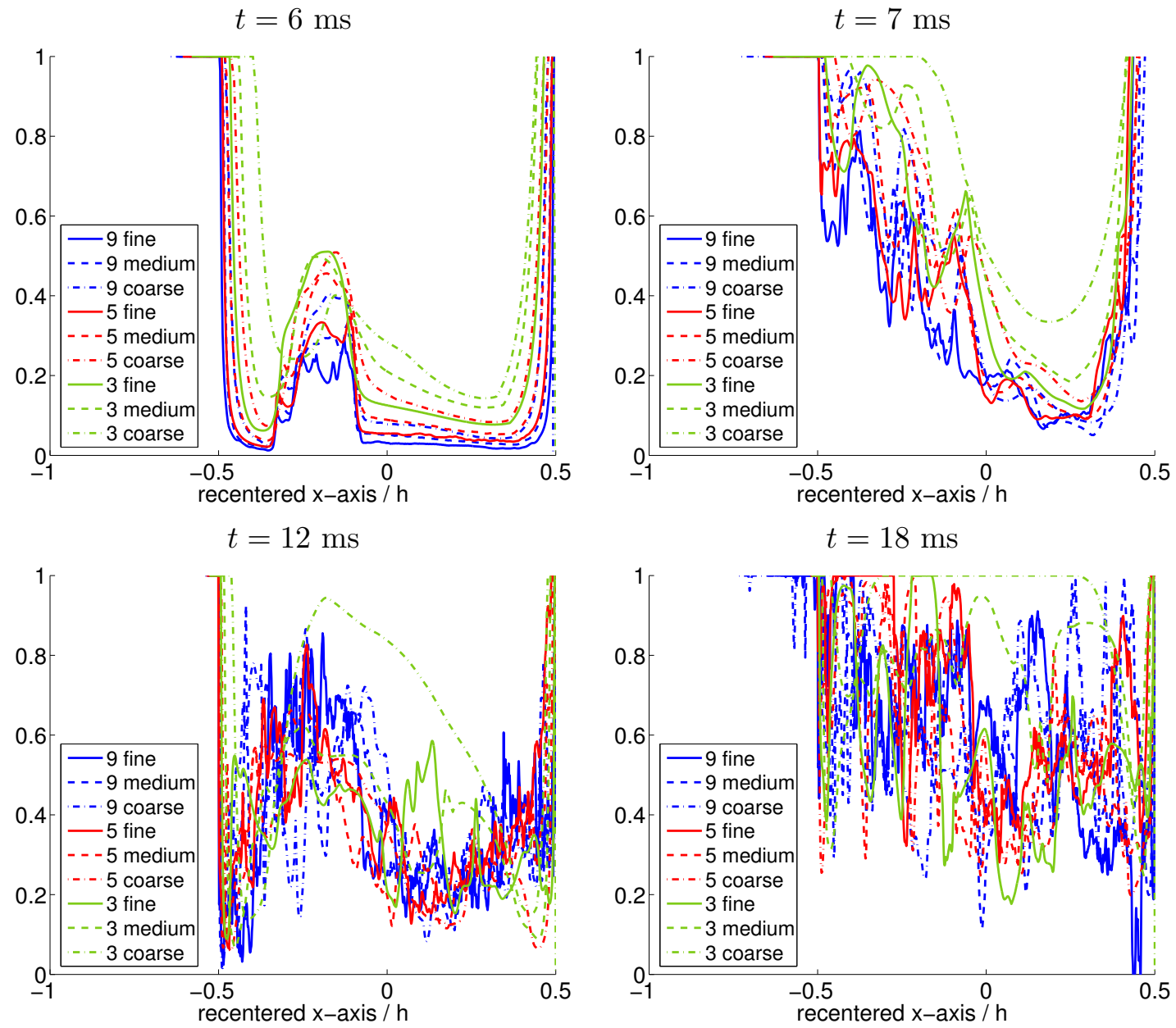

Figure 42: Comparison of the molecular mixing fraction $\xi$ at $t=6,7,12,18$ ms when the order of reconstruction and grid resolution are varied. 
$P_{t}$

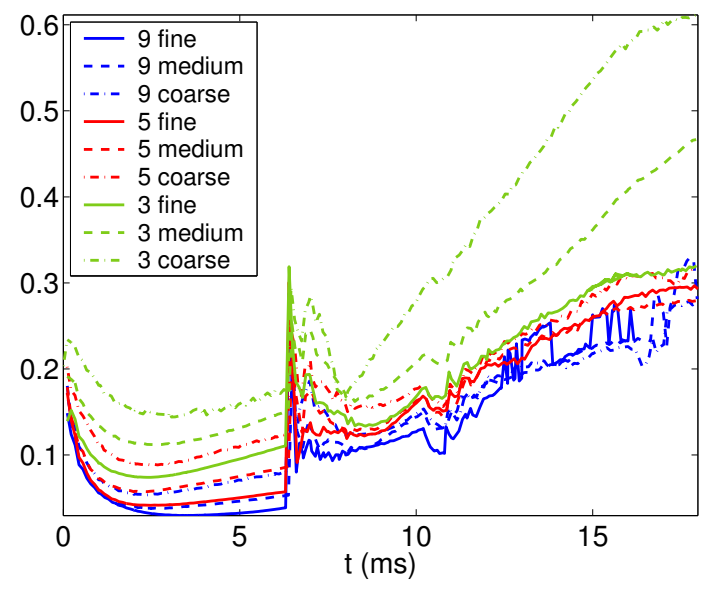

$\Xi$

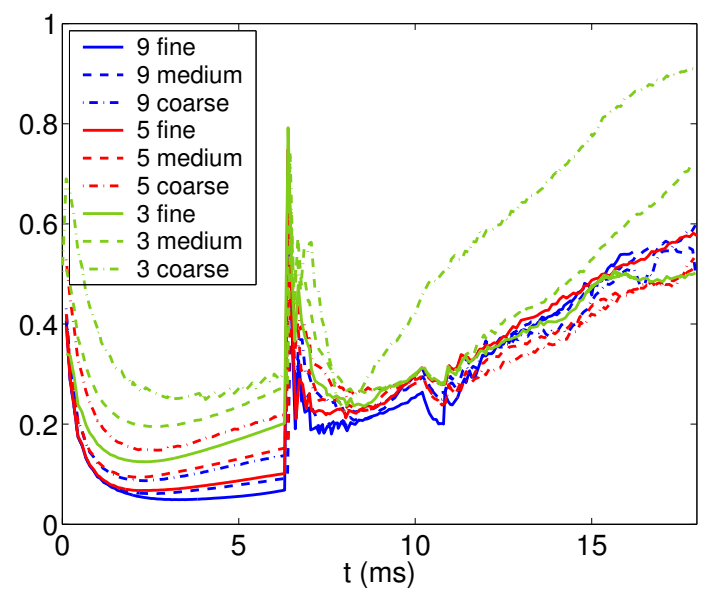

$P_{m}$

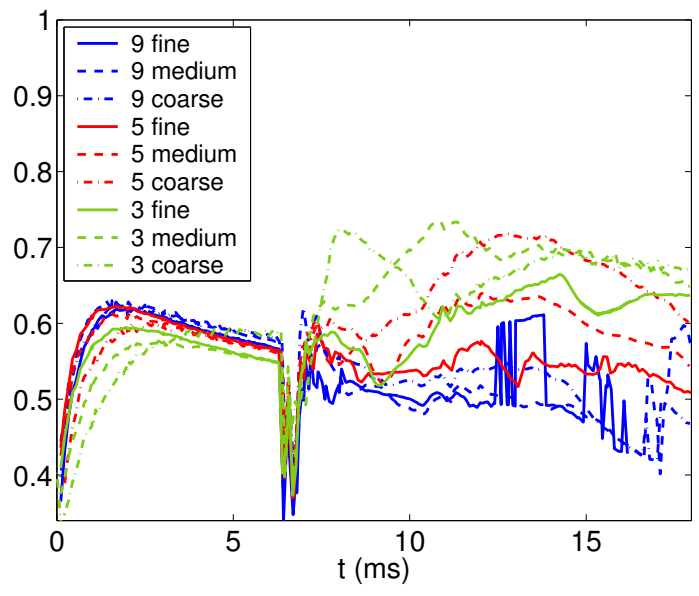

$\Theta$

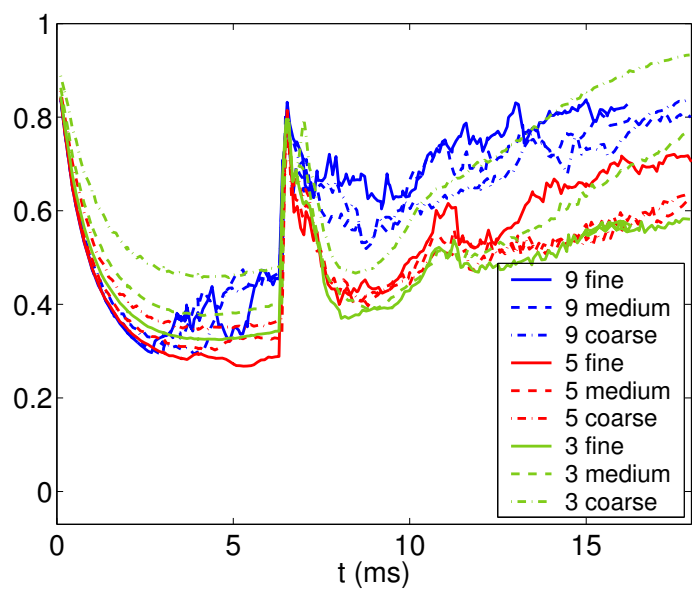

Figure 43: Comparison of the mixing fractions $P_{t}, P_{m}, \Xi$, and $\Theta$ when the order of reconstruction and grid resolution are varied. 
physical insight into the mixedness of the fluids, the results obtained when comparing different orders of reconstruction and different grid resolutions exhibit differences. First consider $\Xi$. The ninth-order simulation at the highest resolution yields the lowest value of $\Xi$ until shortly after reshock when it begins to increase. This is due to the rapid decrease in $P_{m}$ observed above. The rapid increase corresponding to third-order reconstruction at coarse resolution is due to the increase in $P_{t}$ observed above. The plot indicates that for simulations using the same order of reconstruction, $\Xi$ decreases as the resolution increases. Now consider $\Theta$, for which the lowest value is given by the fifth-order reconstruction at fine resolution for times before reshock and by third-order reconstruction at high resolution following reshock. Furthermore, note that no clear trend exists when the grid resolution is varied. For third-order reconstruction, $\Theta$ decreases as the grid resolution is increased, while for ninth-order reconstruction no such trend is observed. These results suggest that global mixing is most sensitive to changes in the grid resolution and the order of reconstruction. Furthermore, the results also indicate that it is difficult to predict a priori how a change in resolution or order of reconstruction affects these mixing quantities.

\subsection{The effect on spectra}

An analysis of the effects of grid refinement on spectra is presented here. Note that as the number of grid points increases, the range of scales resolved increases and, therefore, the tails of the spectra become longer. Also shown are plots of the ratio of each quantity to the value obtained from the ninth-order simulation on the fine grid (the ratio is shown only to the largest value of $k$ obtained from the third-order, coarse grid simulation).

Figure 44 shows a comparison of the fluctuating kinetic energy spectrum $E(k, t)$. At all times considered, differences among all results are apparent across all orders of reconstruction and grid resolutions. This difference in energy becomes more apparent at late time $(t=18 \mathrm{~ms})$ when all of the low-wavenumber modes in the spectrum have been damped out. The levels of energy observed in the fifth-order simulation at fine resolution are similar to the energy observed in the ninth-order simulation at medium resolution. This is further evidence that doubling the resolution gives qualitatively similar results as increasing the order of reconstruction. At intermediate and small scales, the spectra become steeper as the order increases. At late times, the ninth-order simulation on the finest grid has the lowest energy content in the largest scales and the most energy content in the intermediate scales. Figure 45 shows the ratio of fluctuating kinetic energy spectrum from the simulations to the spectrum obtained from the ninth-order simulation on the fine grid. The figure indicates that large differences exist at $t=6 \mathrm{~ms}$, with ratios oscillating above and below one. As time progresses, the oscillations damp out and it becomes clear that the energy content from the ninth-order simulation is the largest. At $t=18$ ms and at large wavenumbers, the energy from the third-order simulation on the coarse grid is larger than the energy in the ninth-order simulations, as indicated by the ratio greater than unity. This phenomenon is due to aliasing which causes the energy to accumulate at large wavenumbers as the Nyquist wavenumber (127) is approached [36, 17].

Figure 46 shows a comparison of the fluctuating enstrophy spectrum $E_{\omega^{\prime \prime} \omega^{\prime \prime}}(k, t)$, which is extremely sensitive to both order and resolution at all times. In particular, as the resolution and the order of reconstruction increase, the fluctuating enstrophy content of both the small and large scales increases. The fluctuating enstrophy spectrum from the ninth-order fine grid simulation differs from that corresponding to the third-order coarse grid simulation by an order of magnitude in the large scales and by several orders of magnitude in the intermediate and 


$$
t=6 \mathrm{~ms}
$$

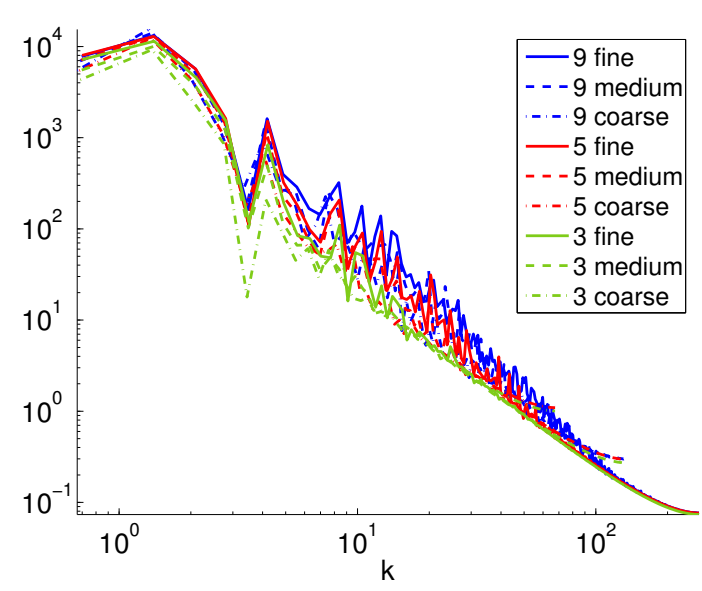

$$
t=12 \mathrm{~ms}
$$

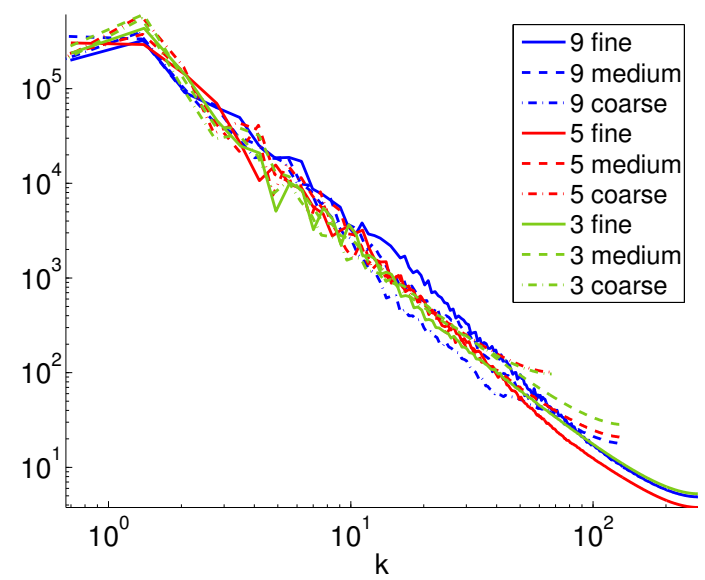

$t=7 \mathrm{~ms}$

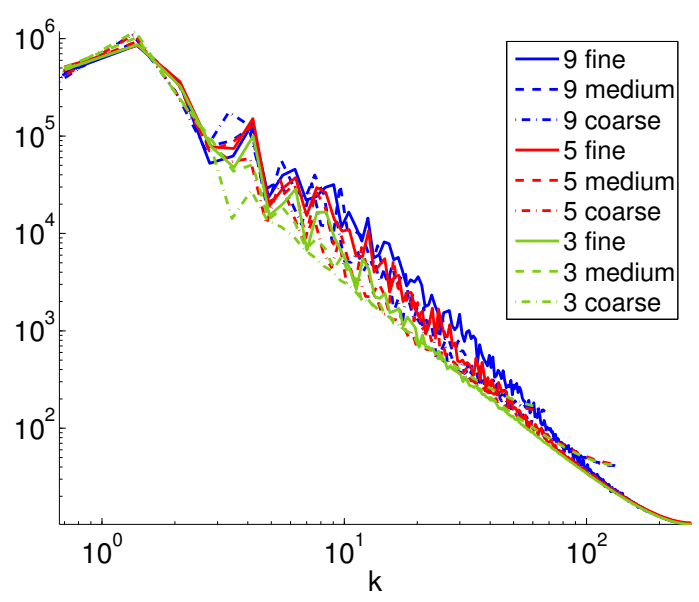

$t=18 \mathrm{~ms}$

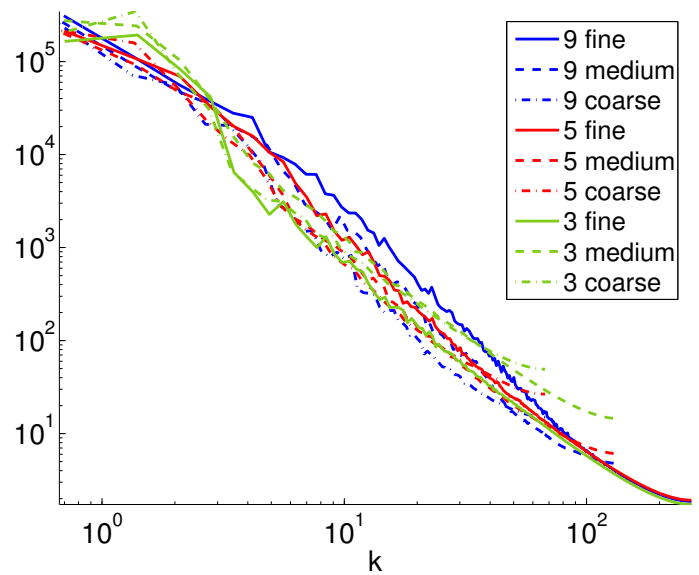

Figure 44: Comparison of the fluctuating kinetic energy spectrum $E(k, t)$ at $t=6,7,12,18 \mathrm{~ms}$ when the order of reconstruction and grid resolution are varied. 

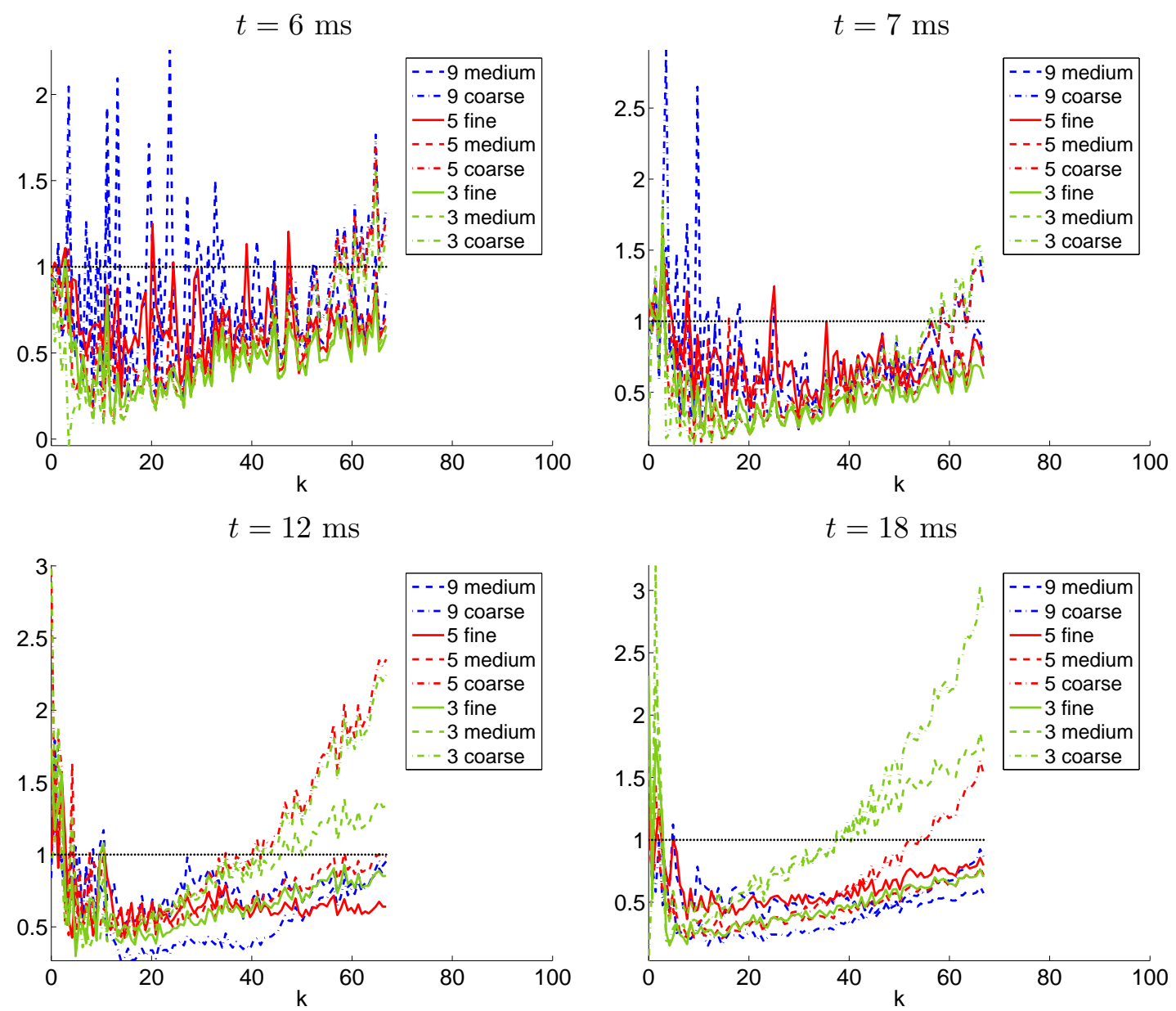

Figure 45: Ratio of the fluctuating kinetic energy spectrum from the simulations to the spectrum from the ninth-order simulation on the fine grid at $t=6,7,12,18$ ms when the order of reconstruction and grid resolution are varied. 

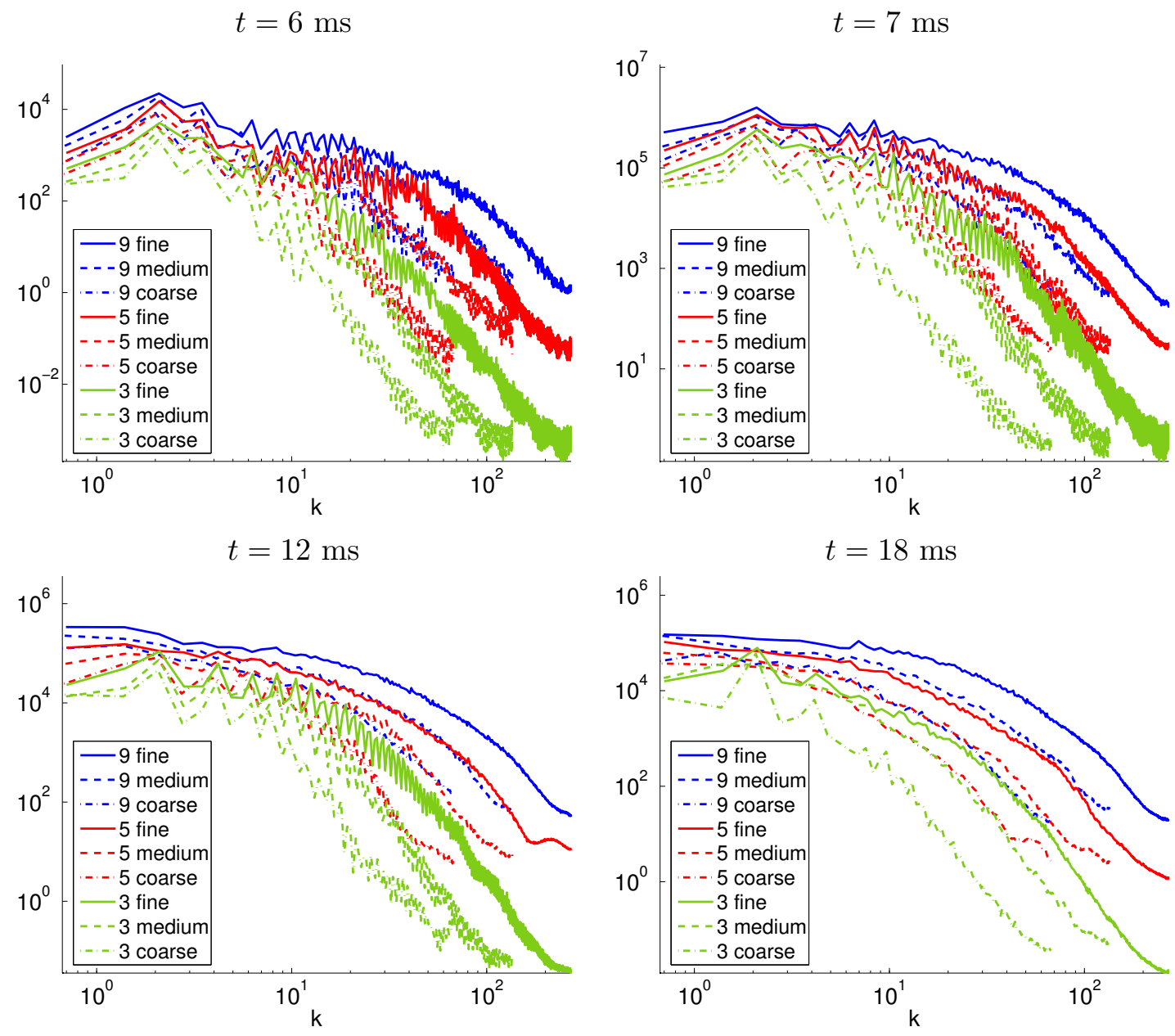

Figure 46: Comparison of the fluctuating enstrophy spectrum $E_{\omega^{\prime \prime} \omega^{\prime \prime}}(k, t)$ at $t=6,7,12,18 \mathrm{~ms}$ when the order of reconstruction and grid resolution are varied. 

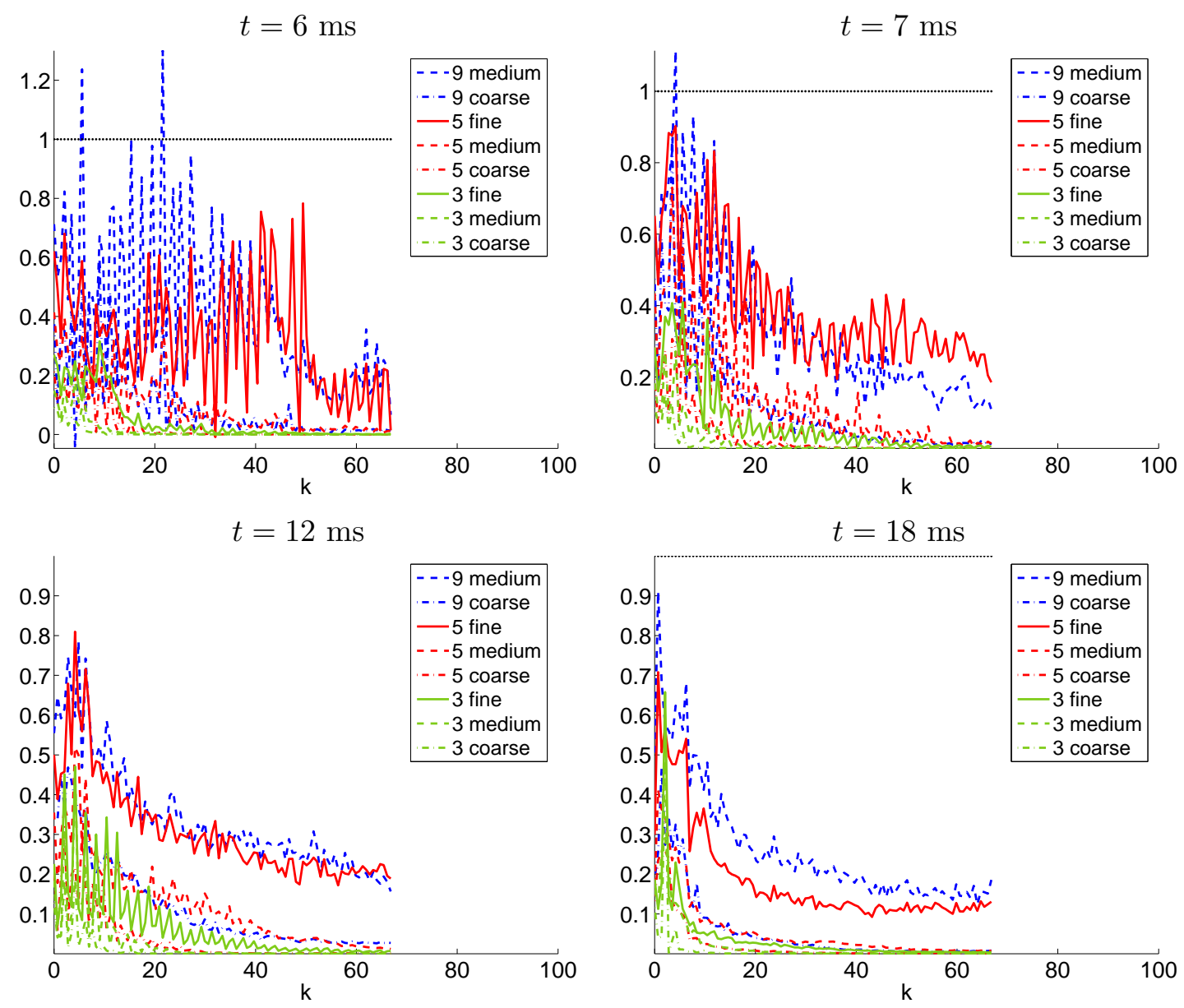

Figure 47: Ratio of the fluctuating enstrophy spectrum from the simulations to the spectrum from the ninth-order simulation on the fine grid at $t=6,7,12,18 \mathrm{~ms}$ when the order of reconstruction and grid resolution are varied. 
small scales. The plots in $\S 5.7$ show that the vorticity corresponding to higher resolutions and higher orders increases in magnitude and is more localized, which is also reflected in the fluctuating enstrophy spectrum. At $t=18 \mathrm{~ms}$, all of the oscillatory modes have been damped out and the spectrum begins decaying. The lower order and lower resolution spectra are much steeper than those at higher order and higher resolution. As the vorticity is constructed from the difference of two velocity gradients, its high-frequency components are more sensitive to numerical damping than are primitive fields such as the velocity. This sensitivity is amplified quadratically as the enstrophy is the square of the vorticity. Figure 47 shows the ratio of fluctuating enstrophy spectrum from the simulations to the spectrum obtained from the ninthorder simulation on the fine grid. The ratio is always below unity for all times and for all simulations, indicating that the fluctuating enstrophy spectrum is very sensitive to the order of reconstruction and grid resolution.

Figure 48 shows the density variance spectrum $E_{\rho^{\prime} \rho^{\prime}}(k, t)$. The density variance spectra are very similar for small wavenumbers, but decrease steeply and rapidly as the wavenumber increases due to the numerical dissipation (which smooths out the fluctuations). As the order is increased or the grid resolution is increased, the numerical dissipation is decreased and the smoothing effects occur at progressively larger wavenumbers. Therefore, the density variance spectrum provides a useful measure of the effects of the numerical dissipation on the flow. The lower order and lower resolution spectra are steeper than those at higher order and higher resolution. Note that the density variance spectrum from the ninth-order simulation on the finest grid apparently exhibits an inertial subrange over slightly more than a decade in wavenumbers at $t=12$ and $18 \mathrm{~ms}$. Figure 49 shows the ratio of the density variance spectrum from the simulations to the spectrum obtained from the ninth-order simulation on the fine grid. At early times, large fluctuations exist near unity indicating a non-uniform modal distribution of the density variance. At later times, as the apparent inertial range develops, the ratio approaches unity, decreasing rapidly as the cutoff wavenumber is reached.

Finally, Fig. 50 shows the pressure variance spectrum $E_{p^{\prime} p^{\prime}}(k, t)$. As the resolution increases, the pressure variance spectrum exhibits differences even in the low-wavenumber range, with higher resolutions and higher orders resulting in increased energy. This is due to the fact that the pressure is obtained from the energy equation with increased amounts of energy deposited on the interface as the resolution and order are increased. The steep decline in the spectrum is also due to the numerical dissipation, which smooths out large-wavenumber fluctuations. The lower order and lower resolution spectra are steeper than those at higher order and higher resolution. Note that the pressure variance spectrum from the ninth-order simulation on the finest grid apparently exhibits an inertial subrange over approximately a decade in wavenumbers at $t=12$ and $18 \mathrm{~ms}$. Figure 51 shows the ratio of the pressure variance spectrum from the simulations to the spectrum obtained from the ninth-order simulation on the fine grid. The figure at $t=6 \mathrm{~ms}$ indicates that increased energy is observed for the simulation on the medium grid at high wavenumbers. In particular, a closer examination of the corresponding plot in Fig. 50 shows that there is a sudden increase in pressure variance similar to what is observed from aliasing. This result is unexpected, and it is not clear what mechanism causes this phenomenon. It is noteworthy that this phenomenon is observed in all of simulations. In particular, the ninth-order simulation on the fine grid also displays an increase in pressure variance in the large wavenumbers. However, the increase occurs at larger wavenumbers than for the ninth-order simulation on the medium grid.

As shown above, the investigation of energy spectra provides quantitative information regarding both the effects of small-scale flow features present at the time of reshock and the 

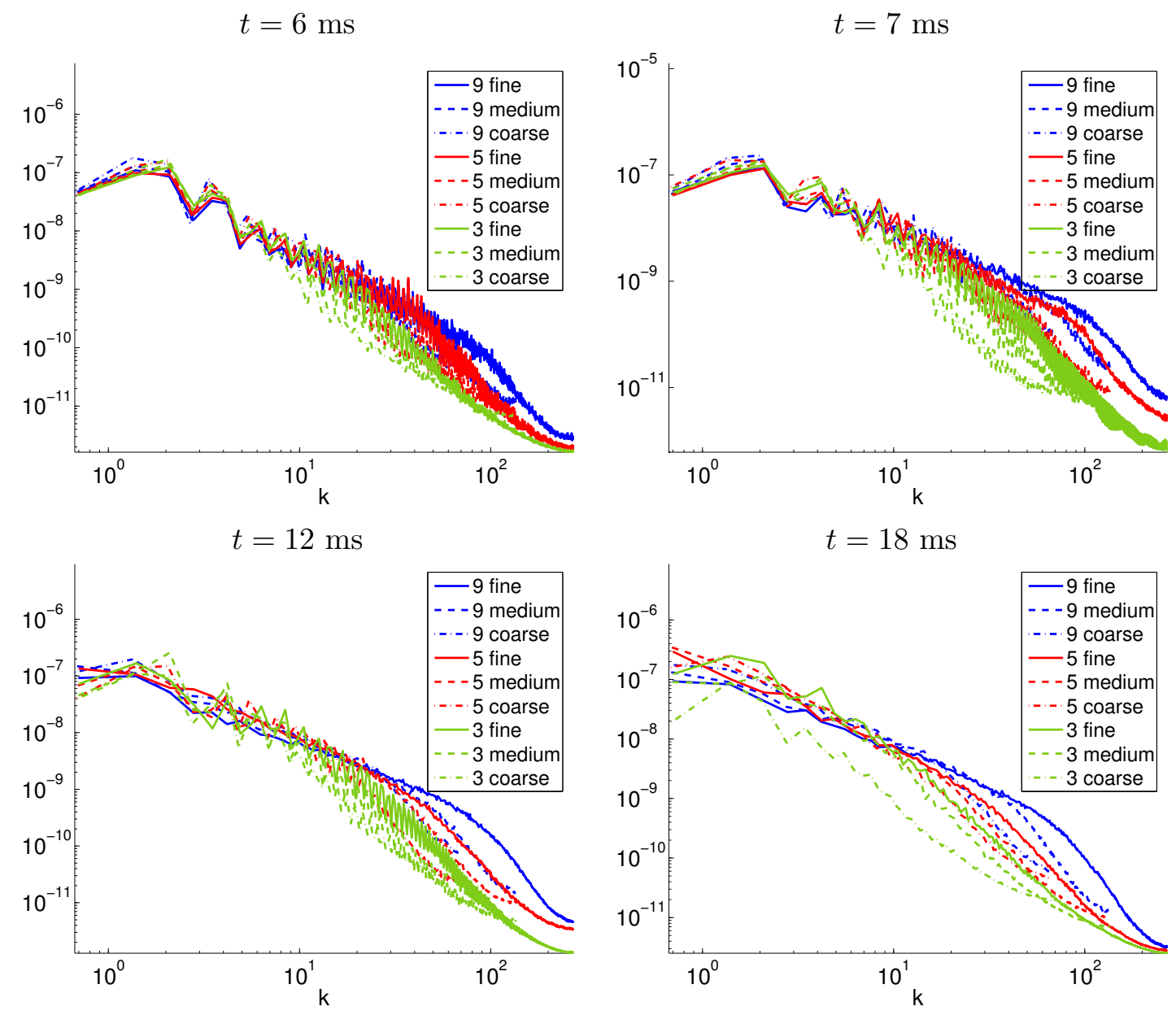

Figure 48: Comparison of the density variance spectrum $E_{\rho^{\prime} \rho^{\prime}}(k, t)$ at $t=6,7,12,18$ ms when the order of reconstruction and grid resolution are varied. 

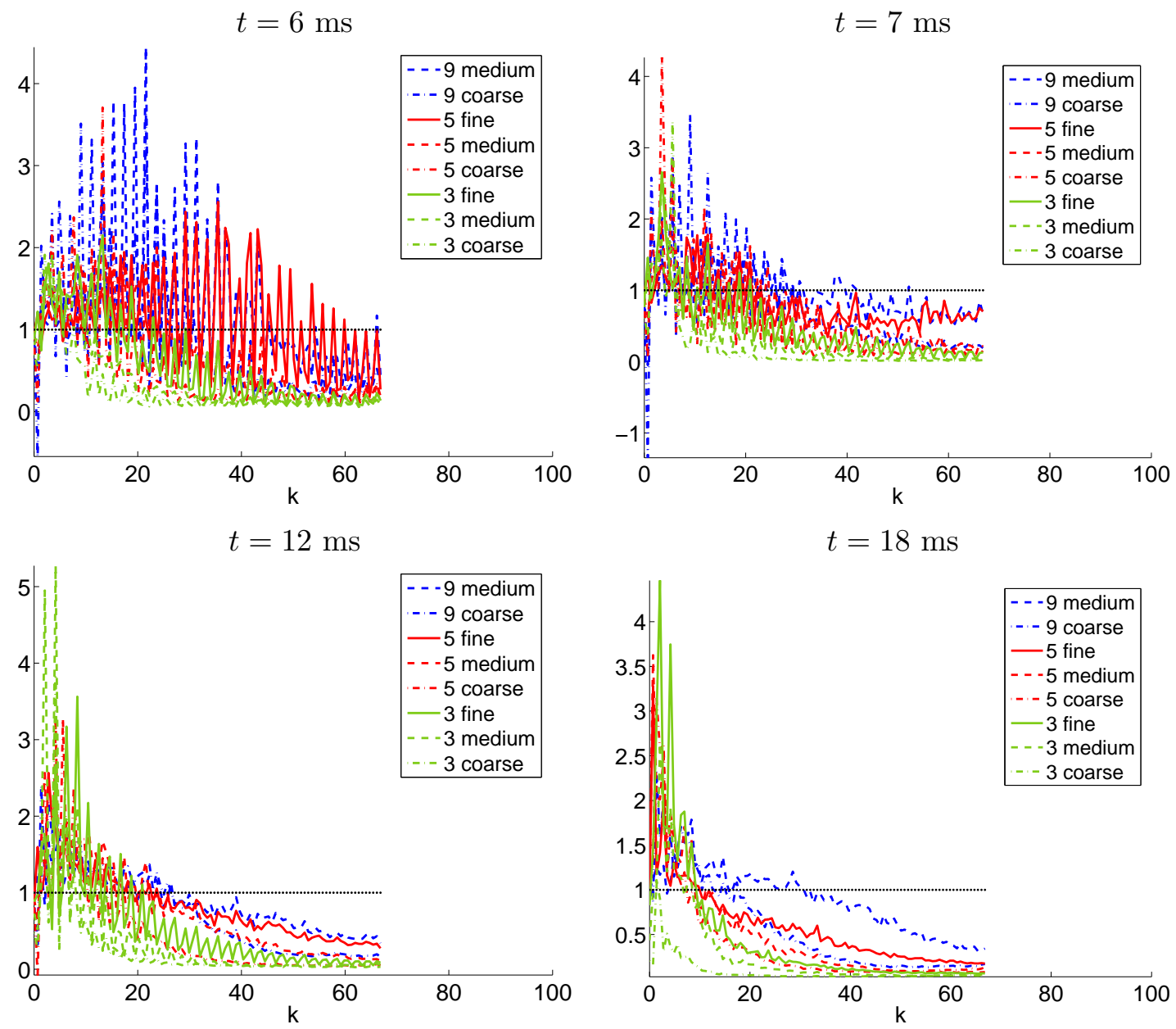

Figure 49: Ratio of the density variance spectrum from the simulations to the spectrum from the ninth-order simulation on the fine grid at $t=6,7,12,18 \mathrm{~ms}$ when the order of reconstruction and grid resolution are varied. 

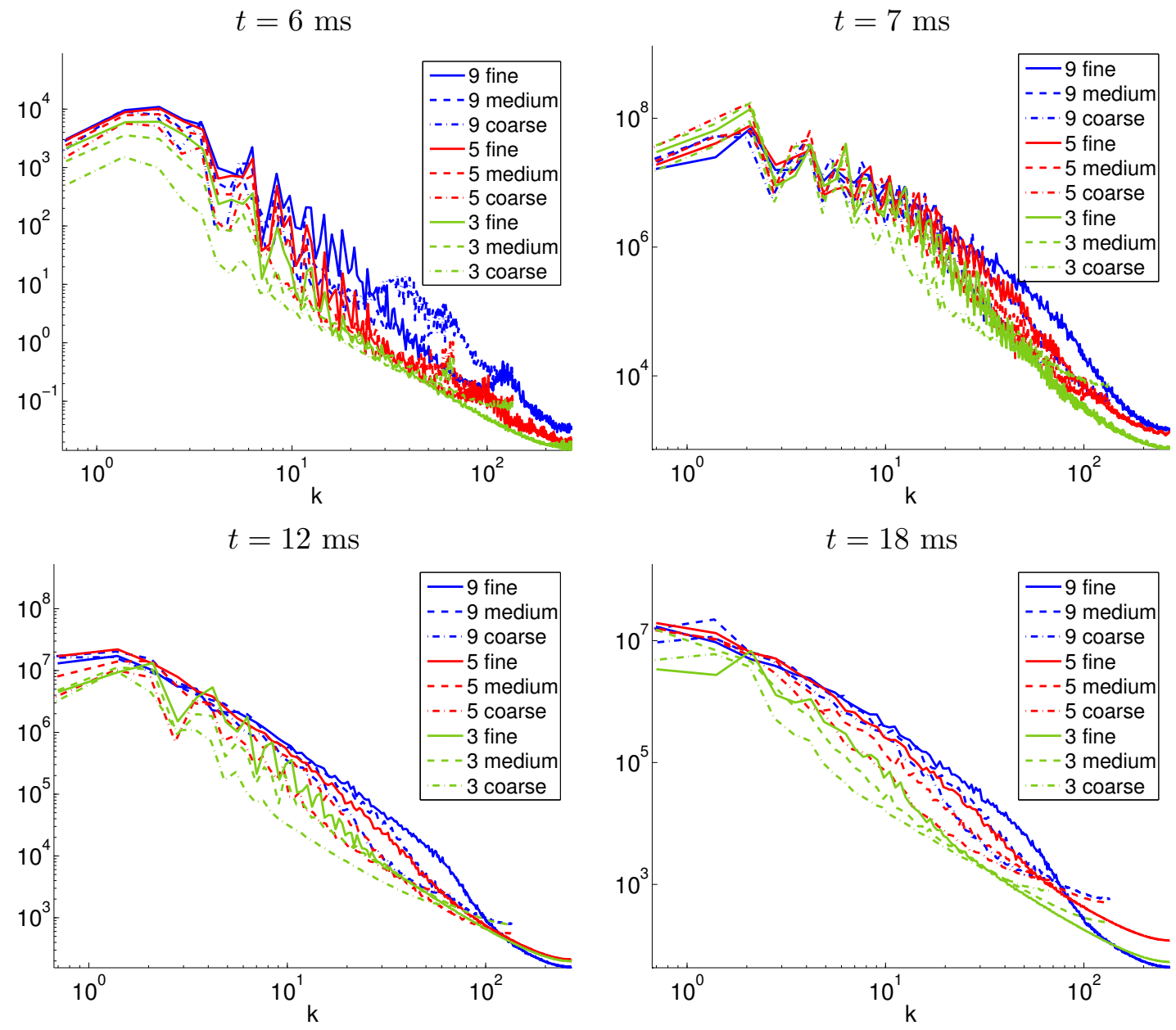

Figure 50: Comparison of the pressure variance spectrum $E_{p^{\prime} p^{\prime}}(k, t)$ at $t=6,7,12,18$ ms when the order of reconstruction and grid resolution are varied. 

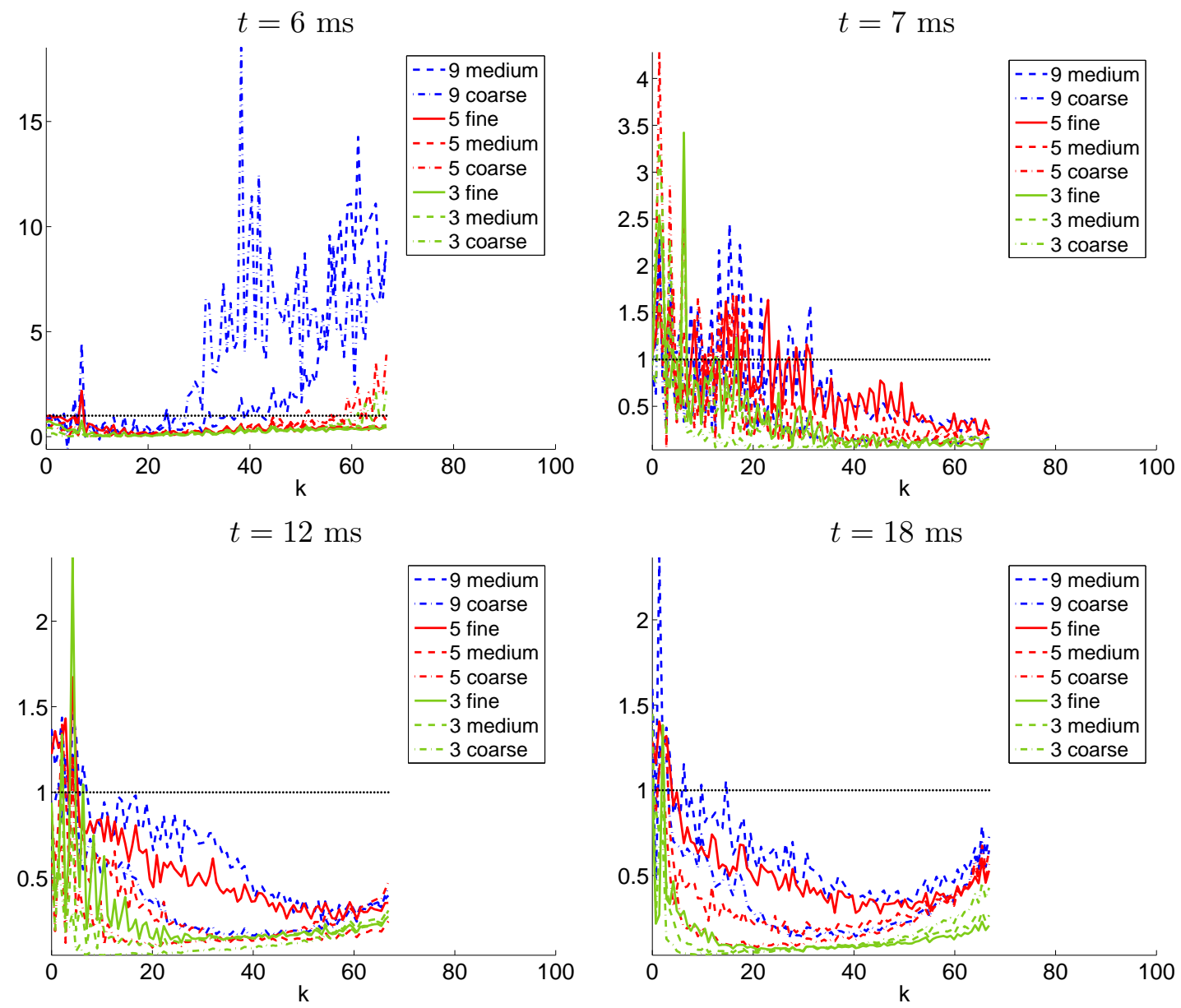

Figure 51: Ratio of the pressure variance spectrum from the simulations to the spectrum from the ninth-order simulation on the fine grid at $t=6,7,12,18 \mathrm{~ms}$ when the order of reconstruction and grid resolution are varied. 
effects of intrinsic numerical dissipation of an algorithm. The effects of small-scale flow features and fluctuations are captured by the energy spectra. In particular, the jump in magnitude across different resolutions and orders of reconstruction is a direct consequence of the presence of small-scale flow features. The effects of numerical viscosity can be best captured by the density variance spectrum, in which the steep decline at high-wavenumbers is a direct effect of numerical dissipation smoothing out small-scale fluctuations. As observed from the ratio of each spectrum from the simulations with different orders and resolutions to the spectrum obtained from the ninth-order simulation on the fine grid, there is no apparent 'convergence' of the spectra: this indicates that quantities sensitive to the small-scale structure of the flow do not generally exhibit 'convergence' while quantities determined primarily by the largescale structures appear to be 'converged' at sufficiently high order of reconstruction and grid resolution, i.e., the mixing layer amplitude or width (as shown in $\S 5.2$ ).

\subsection{The effect on statistics}

An investigation of the effects of grid refinement and order of reconstruction on the statistics (i.e., wavenumber-integrated energy spectra) is presented here.

Figure 52 shows the fluctuating kinetic energy and its components. As the order of reconstruction and resolution are increased, the overall energy increases before reshock. However, this is no longer the case following reshock. For example, consider $E_{u^{\prime \prime} u^{\prime \prime}}(t)$. The ninth-order result at coarse resolution has the lowest energy, which can be explained by recalling that the mixing layer width obtained from the ninth-order simulation is larger than that obtained from the third-order simulation, resulting in less overall energy. Also note that $E_{v^{\prime \prime} v^{\prime \prime}}(t)$ is much more sensitive to the spatial resolution and to the order of reconstruction than $E_{u^{\prime \prime} u^{\prime \prime}}(t)$, both before and after reshock (particularly at late times). Following reshock, the simulations at lowest resolution are no longer the simulations with the least energy content: this is again due to the reduced mixing layer width observed for coarser simulations, which yields increased levels of energy.

The subtle differences observed for the kinetic energy are no longer apparent when considering the fluctuating enstrophy $E_{\omega^{\prime \prime} \omega^{\prime \prime}}(t)$. Figure 52 shows that the enstrophy increases as the order and spatial resolution increase. The enstrophy differentiates between the orders of reconstruction and grid resolutions, and the enstrophies from simulations with increased grid resolution or higher order of reconstruction are very similar. Just before reshock, as well as for all times following reshock, the fluctuating enstrophy from the ninth-order fine grid simulation differs from that obtained from the third-order coarse grid simulation by an order of magnitude.

The density variance $E_{\rho^{\prime} \rho^{\prime}}(t)$ shown in Fig. 52 exhibits agreement once the order and grid resolution are sufficiently high. This result is consistent with the previous observation that the density variance spectrum does not significantly change as a function of grid resolution and order of reconstruction, with the exception of a steep decline at large wavenumbers. Contributions from the small scales (large wavenumbers) are not weighted heavily in the computations of statistics, which is consistent with the small changes. The density variance is rapidly damped in the third-order, coarse and medium grid simulations. The pressure variance $E_{p^{\prime} p^{\prime}}(t)$ shown in Fig. 52 exhibits little sensitivity to changes in the order of reconstruction and grid resolution. 

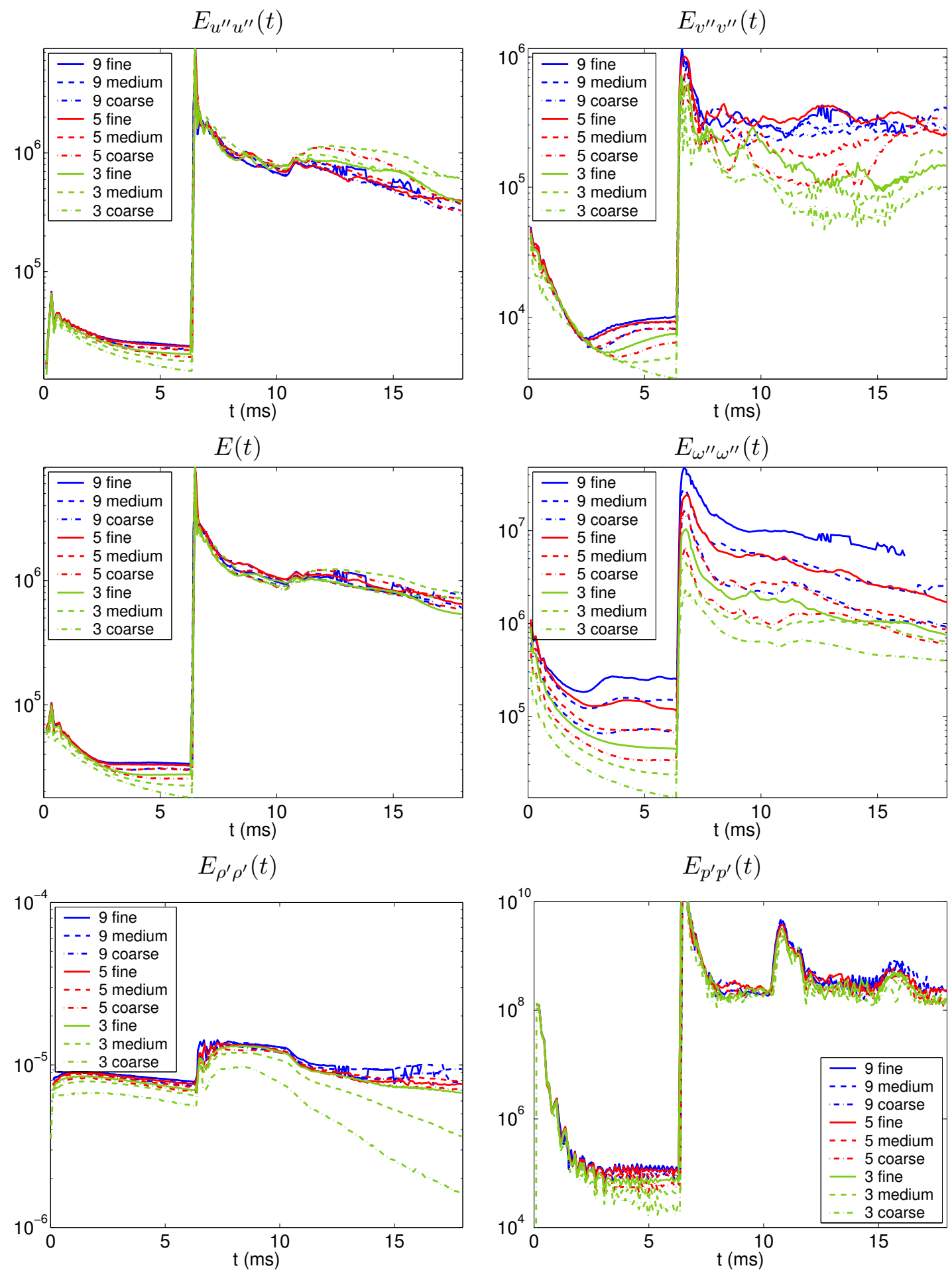

Figure 52: Comparison of $E_{u^{\prime \prime} u^{\prime \prime}}(t), E_{v^{\prime \prime} v^{\prime \prime}}(t), E(t), E_{\omega^{\prime \prime} \omega^{\prime \prime}}(t), E_{\rho^{\prime} \rho^{\prime}}(t)$, and $E_{p^{\prime} p^{\prime}}(t)$ when the order of reconstruction and grid resolution are varied. 


\subsection{Temporal progression of density and vorticity}

Presented here is the evolution of the density and vorticity fields at intervals of $1 \mathrm{~ms}$ resulting from the single-mode, two-dimensional Richtmyer-Meshkov instability with reshock obtained from WENO simulations with third-, fifth-, and ninth-order of reconstruction and grid resolutions of 128, 256, 512 points per initial perturbation wavelength. The following convention is adopted in the presentation of the results. The top, middle, and bottom rows show the results of the ninth-, fifth-, and third-order simulations, respectively. The left, middle, and right columns display results obtained with increasing spatial resolution. The purpose of this section is to qualitatively illustrate how the evolution of the instability changes as the numerical parameters are varied. The figures also aid in interpreting the results in previous sections.

At early times, the interface is more diffused for the lower order and lower resolution. As time evolves, a qualitative correspondence in both the density and vorticity occurs between the simulations on the diagonal, i.e., results obtained using a $(2 n-1)$-th order method with $n=2,3$ and $N$ grid points per initial perturbation wavelength are similar to those obtained using a $(2 n-1)$-th order method with $n=3,5$ and $N / 2$ grid points per initial perturbation wavelength, respectively. The higher-order and finer grid results show sharper, smaller-scale features with well-defined roll-ups (more concentrated vortex cores), as well as thinner diffusion layers. The vortex cores are larger in the lower order and lower resolution simulations.

The amplitudes from the higher order and higher resolution simulations are slightly larger than those from the lower order and lower resolution simulations before reshock. After reshock, the difference in amplitude increases due to the mechanisms described in $\S 5$. The third-order simulations remain highly diffuse for all grid resolutions, even at late times following reshock. As shown in the figures with $t \geq 7 \mathrm{~ms}$, the third-order simulations retain significant coherency and symmetry following reshock. The third-order results are characteristic of simulations with a high degree of numerical diffusion, as well as simulations that are under-resolved.

By $t=6 \mathrm{~ms}$, the medium grid ninth-order simulation exhibits additional small-scale structure within the roll-ups. This additional structure has also been reported by several investigators using the piecewise-parabolic method (PPM) [105, 82], and is apparently a manifestation of a physical process observed in experiments [44, 78, 23]. By contrast, the fine grid ninth-order simulation begins to show asymmetrical and fragmented structure within the roll-ups, as well as on the interface. Such structure was also observed in ninth- and eleventh-order numerical simulations at high resolutions conducted at Brown University; it is speculated that as the roll-ups form, the interface separating the two gases is stretched increasingly thinner and the differences in flow velocities across the interface, along with the numerical perturbations, cause the formation of an instability similar to Kelvin-Helmholtz instability [27]. It is believed that this is a manifestation of a numerical instability due to the small numerical diffusion that is unable to provide sufficient regularization of the Euler equations as the grid Reynolds number $[95,81]$

$$
R e_{\Delta x}=\frac{c_{s} \Delta x}{\nu_{n u m}}
$$

increases (where $\nu_{\text {num }}$ is some parameterization of the numerical viscosity of the method and $c_{s}$ is the speed of sound). It would be of interest to perform a grid convergence study using explicit molecular dissipation and diffusion to determine whether this fragmentation persists. Following reshock the ninth-order simulations exhibit the most small-scale, disordered structure with significant fragmentation of the density and the vorticity, and are most reminiscent of the experimental PLIF images in Collins and Jacobs. By contrast, the lower order and lower 
resolution simulations continue to exhibit relatively ordered structure, even following reshock.

This study constitutes the first systematic investigation of the effects of numerical simulation parameters on the instability evolution. The results were also obtained for a twodimensional Eulerian flow. Therefore, the differences in instability evolution depend solely on the numerical dissipation of the method $\nu_{n u m}$ and not on a physical dissipation or vortex stretching in three-dimensional simulations. In part four of this report [59] three dimensional simulations are considered and a systematic investigation of two- and three-dimensional effects is discussed. 

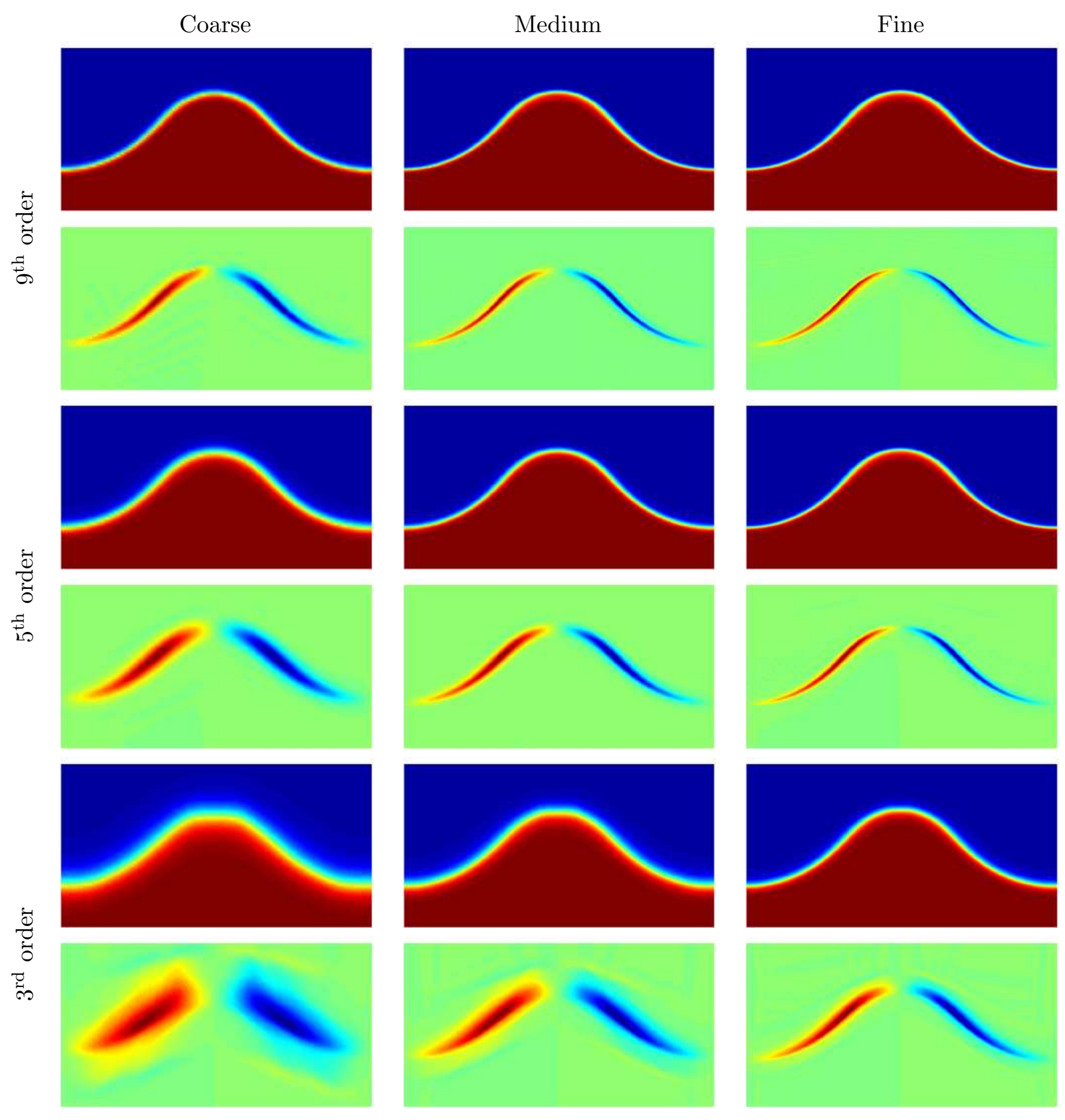

Figure 53: Density and vorticity at $t=1 \mathrm{~ms}$. 

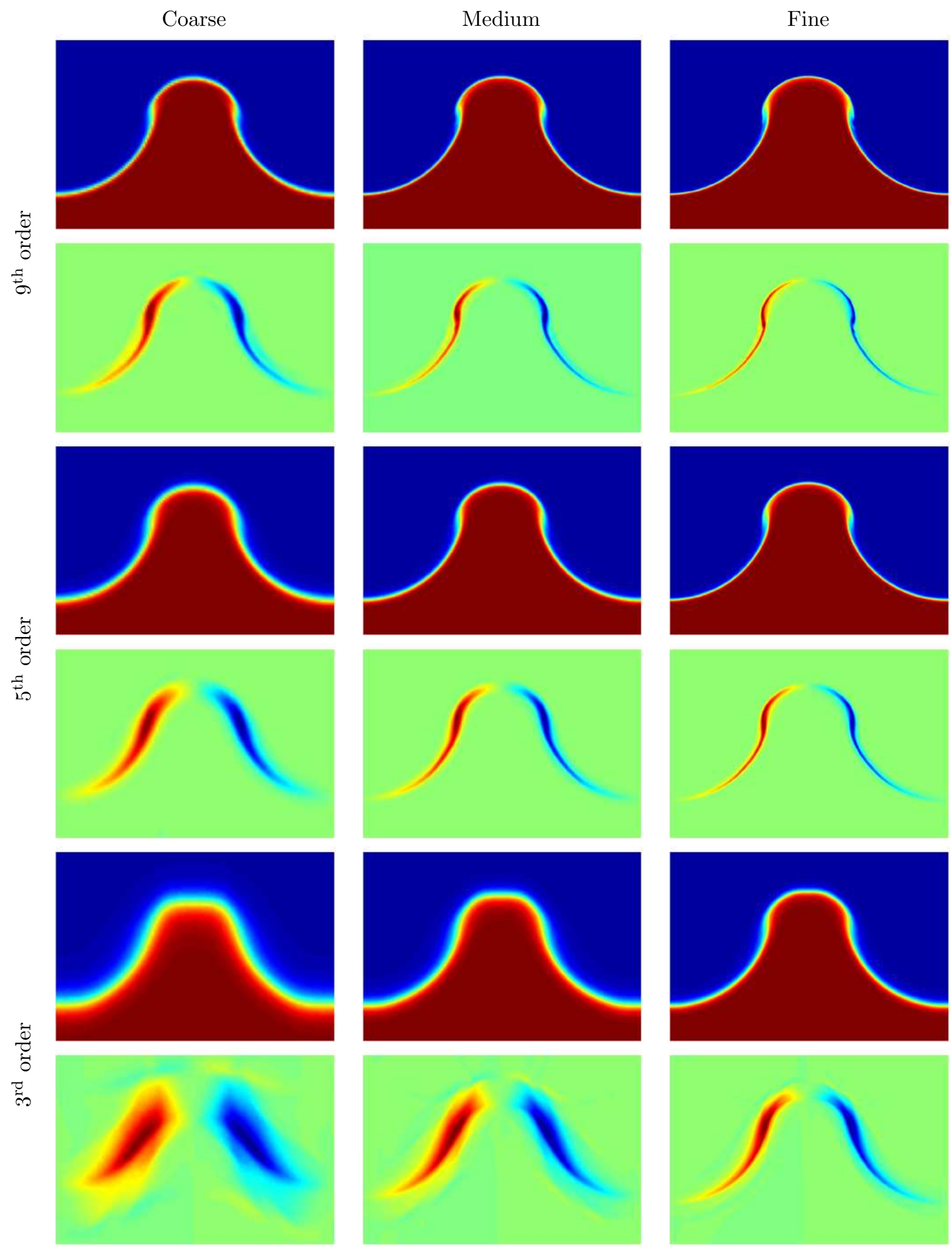

Figure 54: Density and vorticity at $t=2 \mathrm{~ms}$.
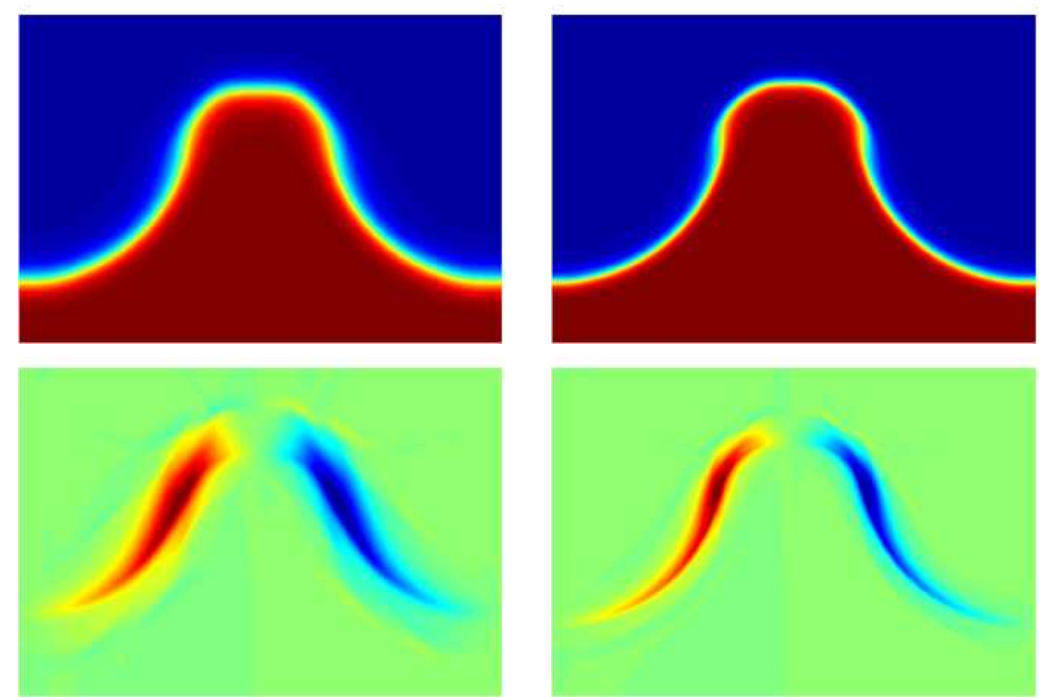

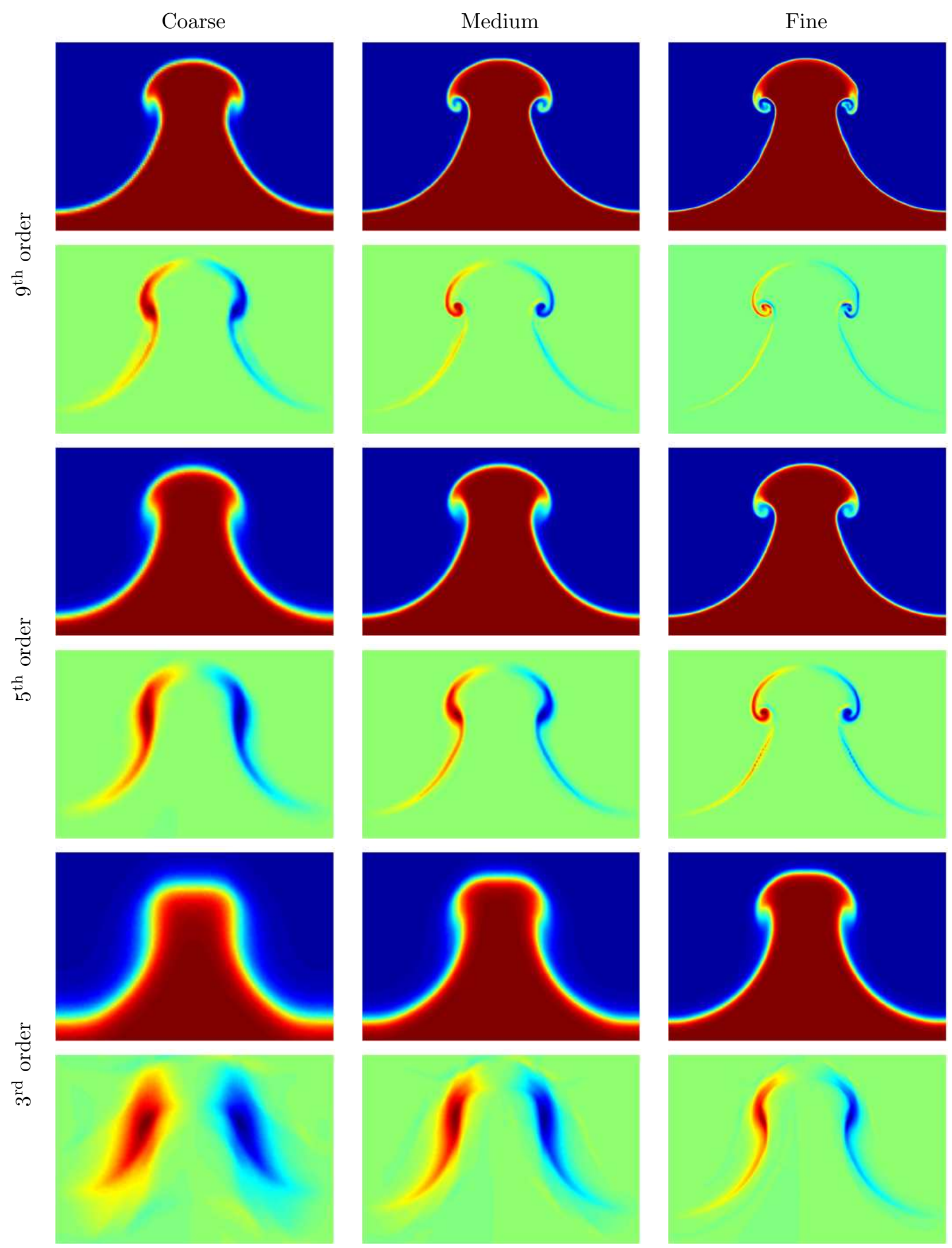

Figure 55: Density and vorticity at $t=3 \mathrm{~ms}$. 

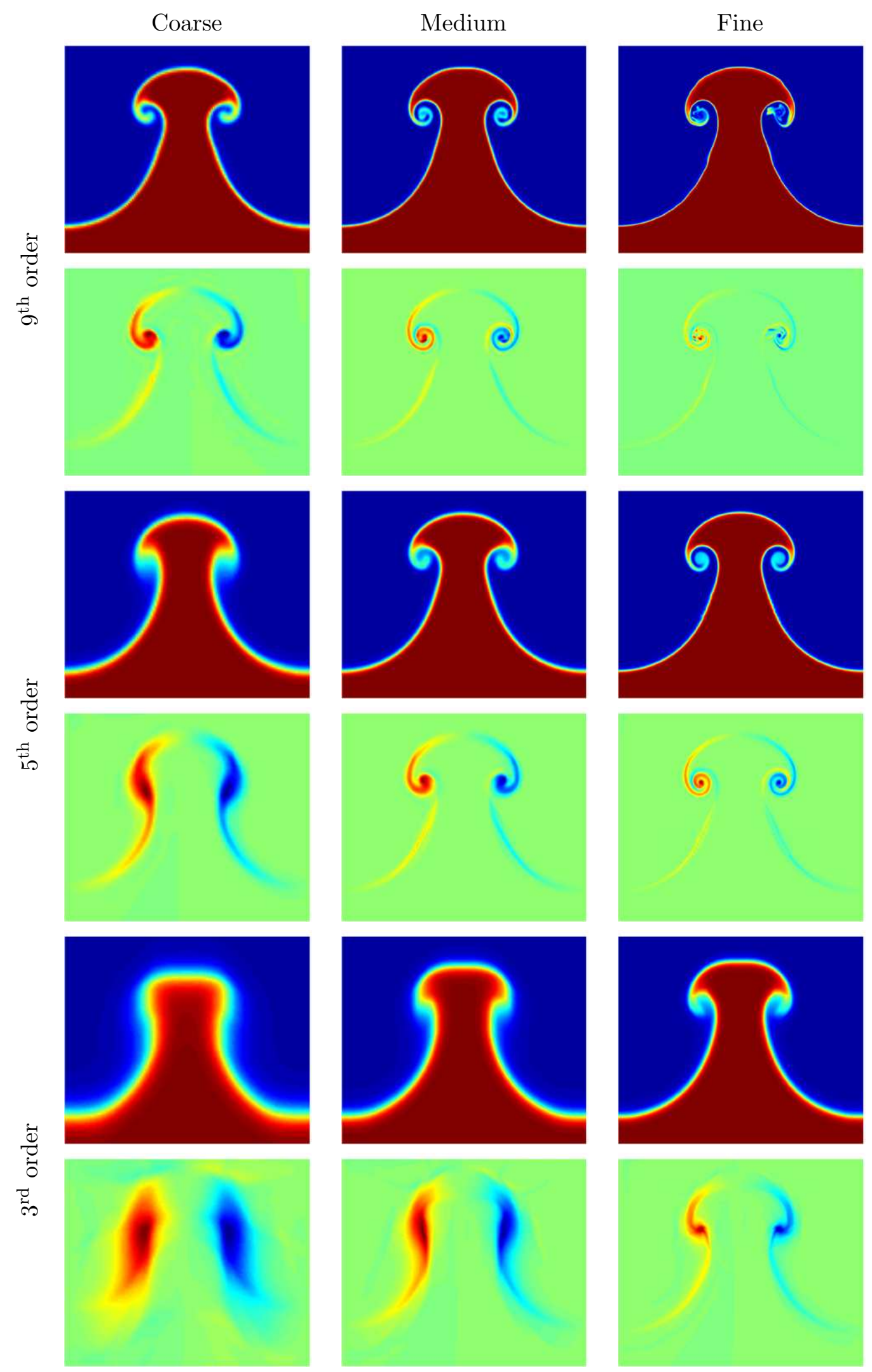

Figure 56: Density and vorticity at $t=4 \mathrm{~ms}$. 

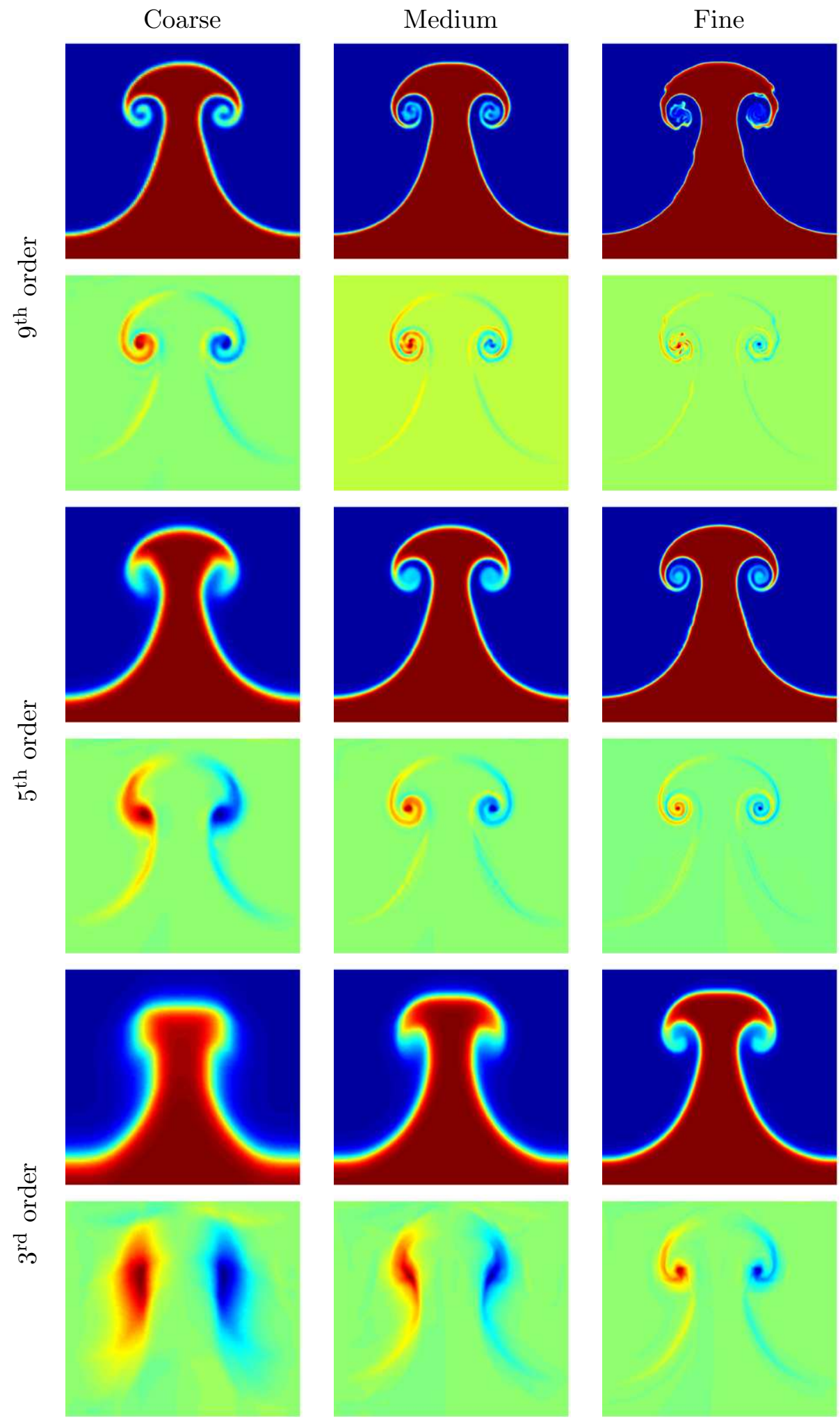

Figure 57: Density and vorticity at $t=5 \mathrm{~ms}$. 

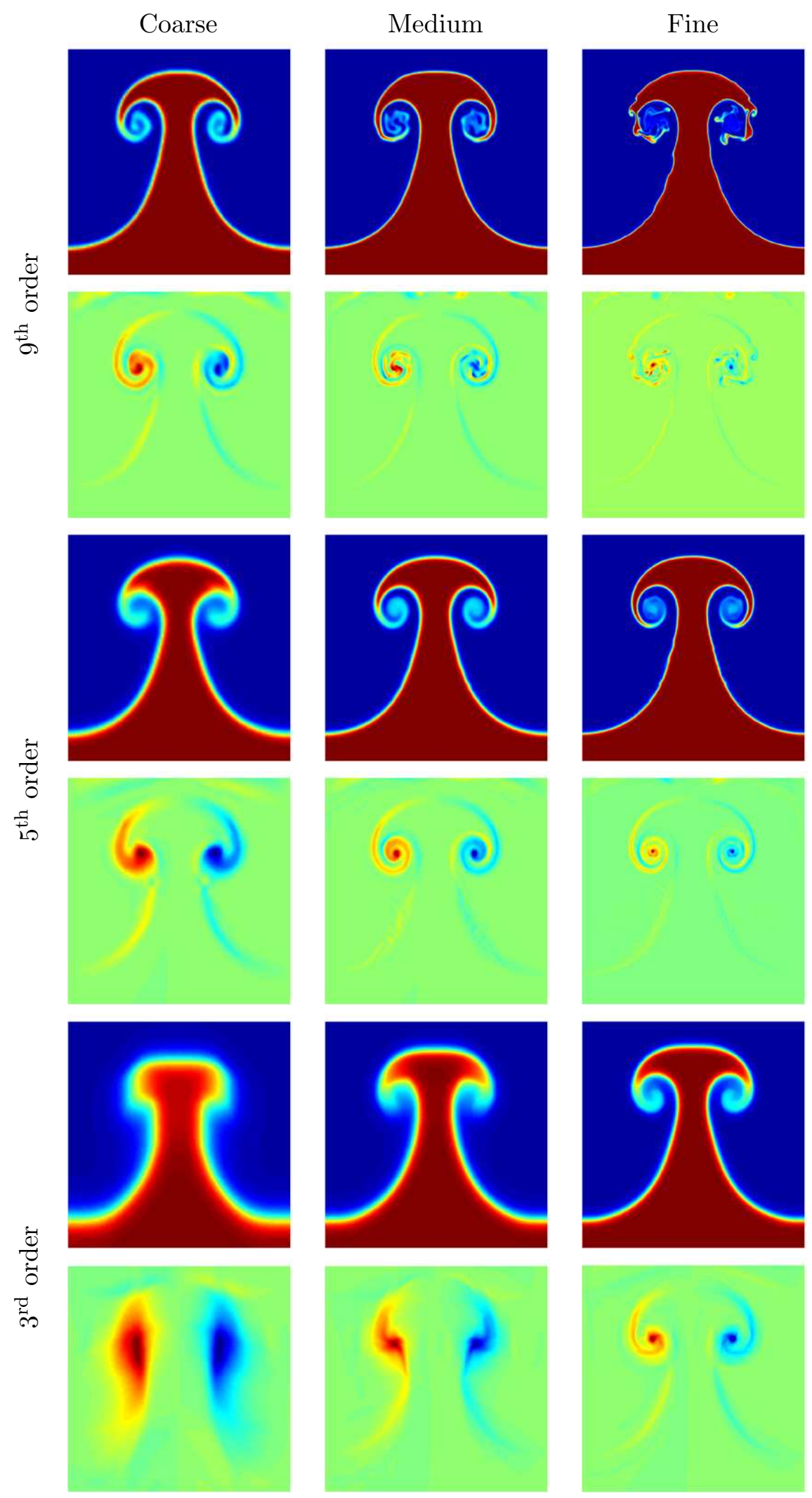

Figure 58: Density and vorticity at $t=6 \mathrm{~ms}$. 


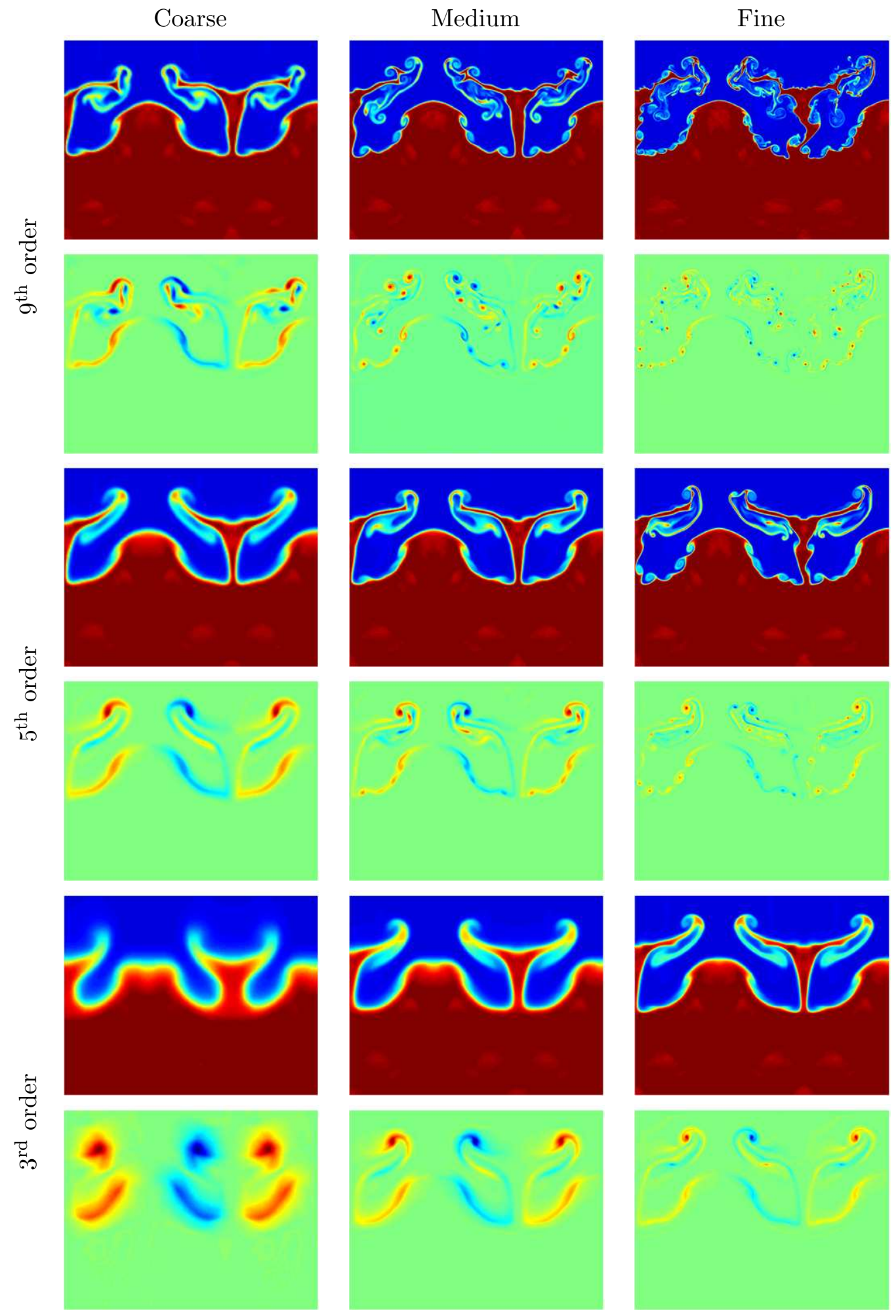

Figure 59: Density and vorticity at $t=7 \mathrm{~ms}$. 

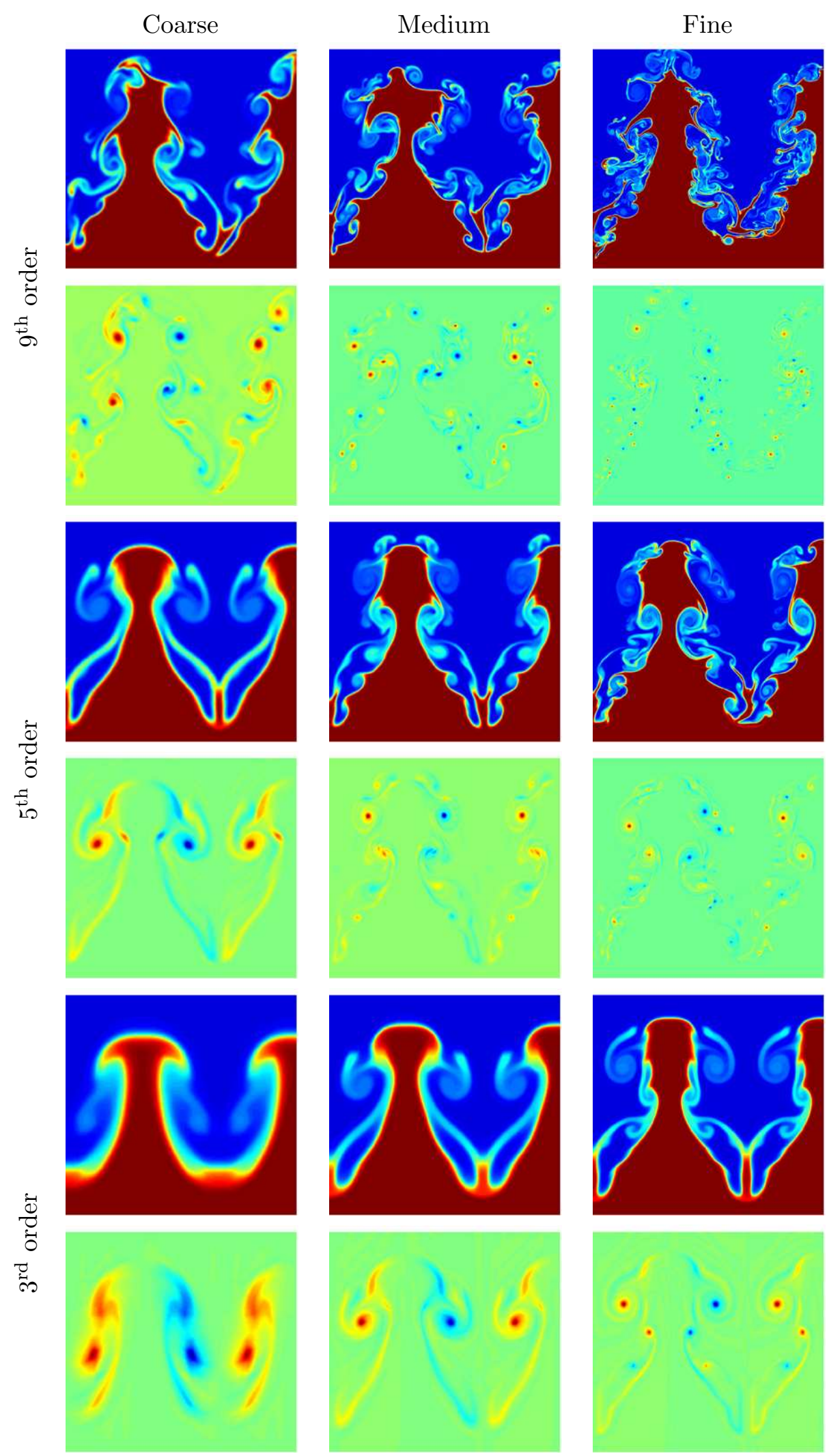

Figure 60: Density and vorticity at $t=8 \mathrm{~ms}$. 


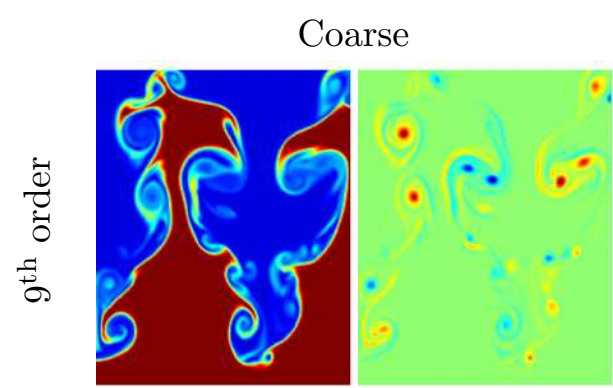

Medium
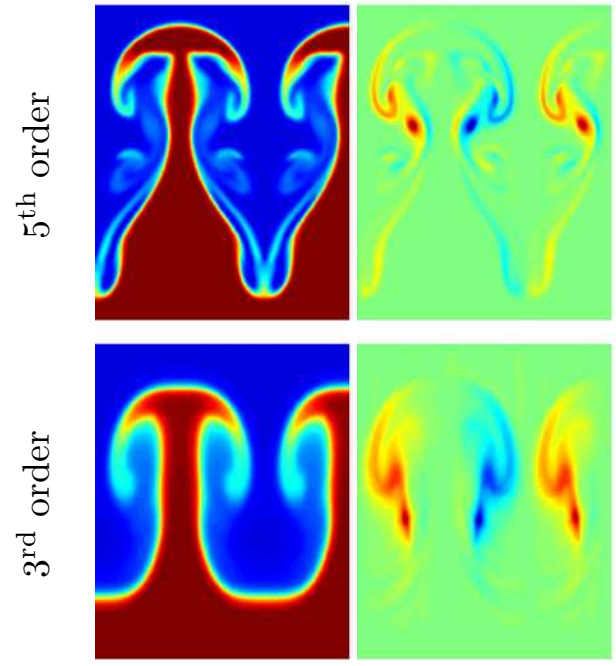
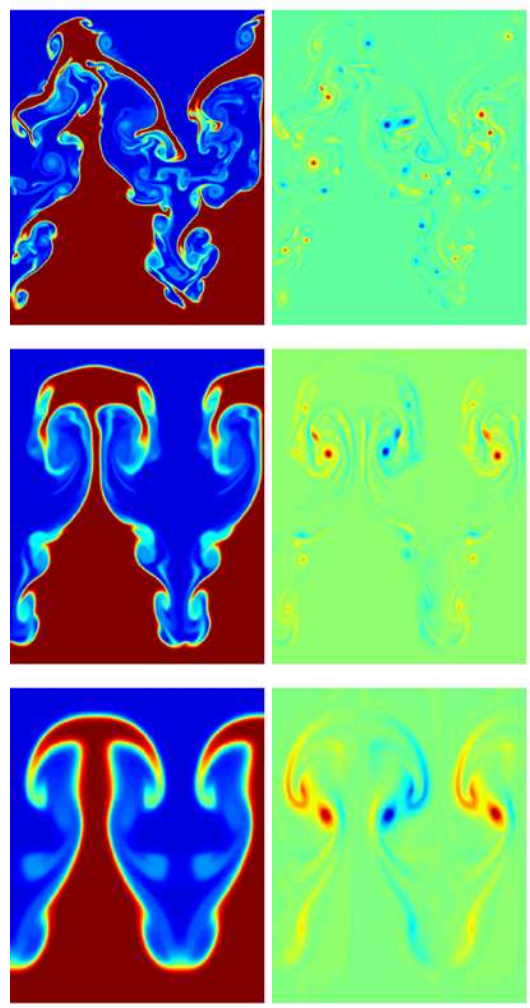

Fine
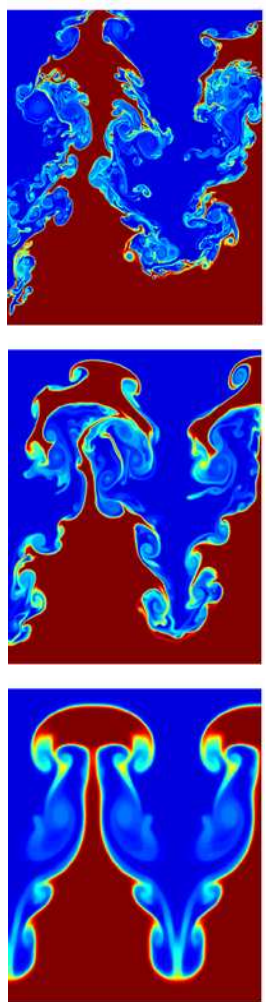

Figure 61: Density and vorticity at $t=9 \mathrm{~ms}$. 

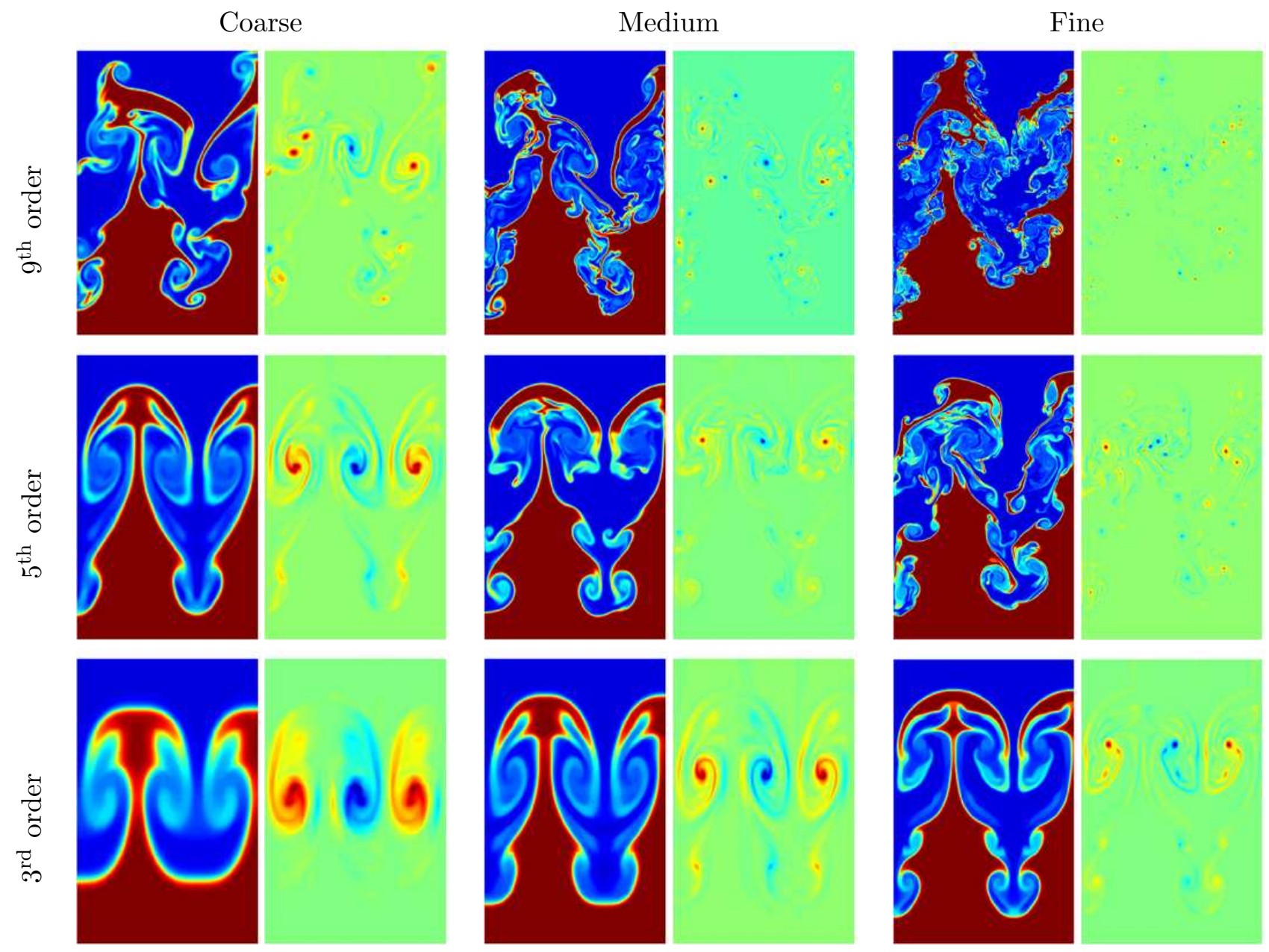

Figure 62: Density and vorticity at $t=10 \mathrm{~ms}$. 

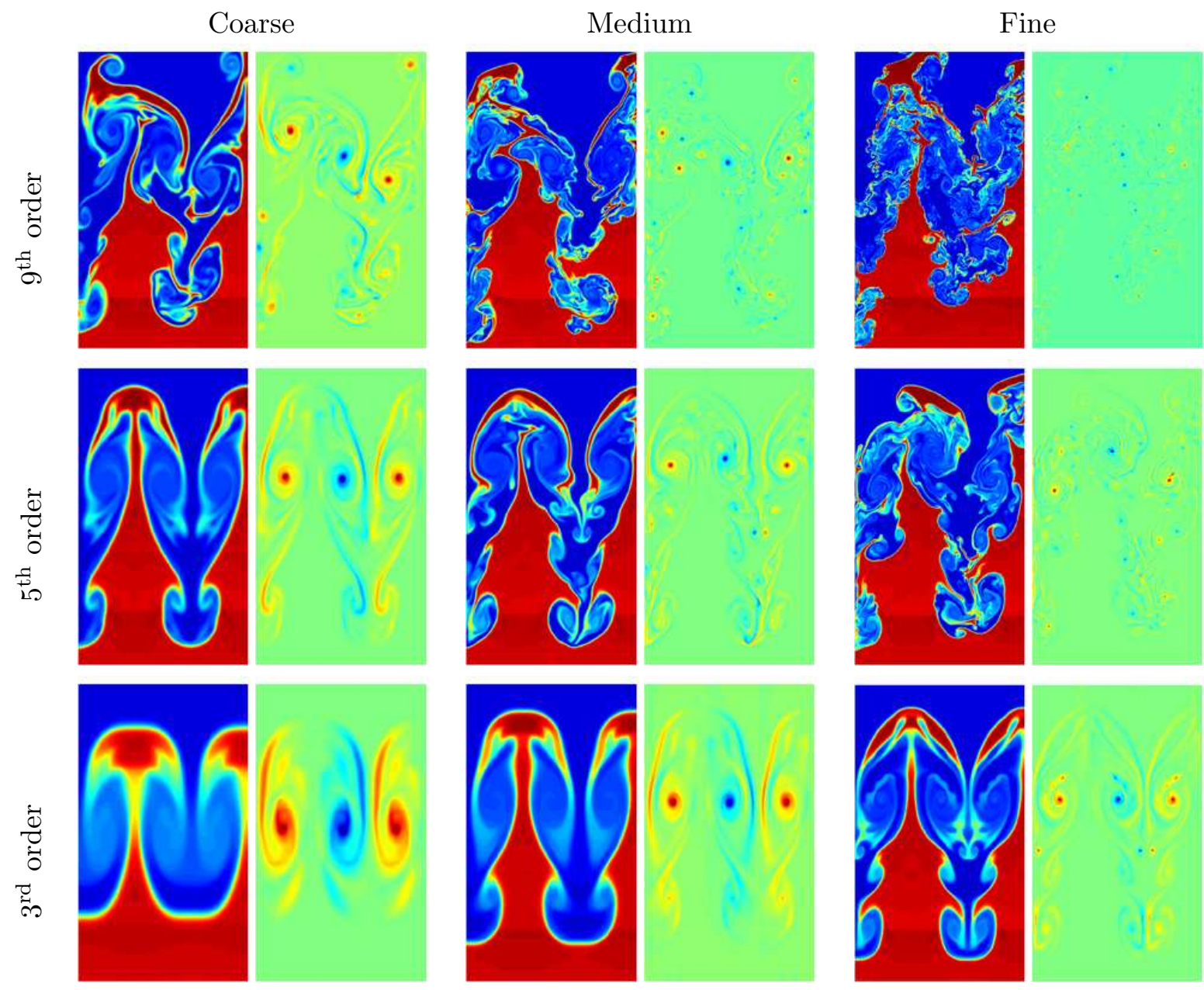

Figure 63: Density and vorticity at $t=11 \mathrm{~ms}$. 

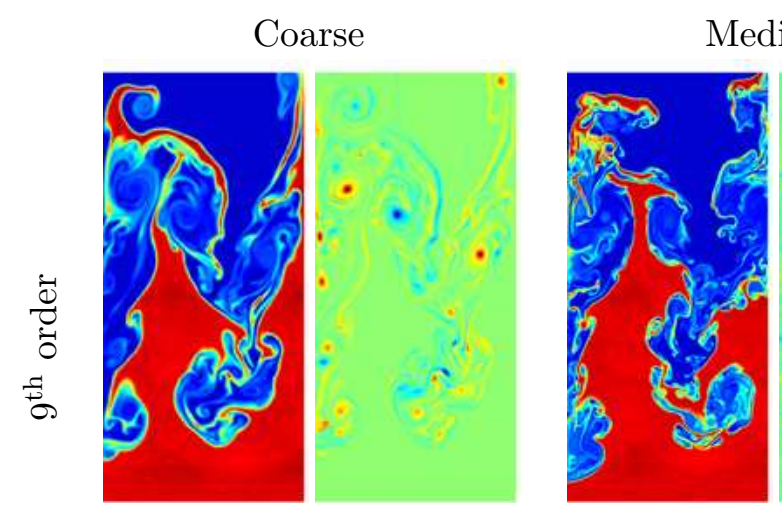

Fine
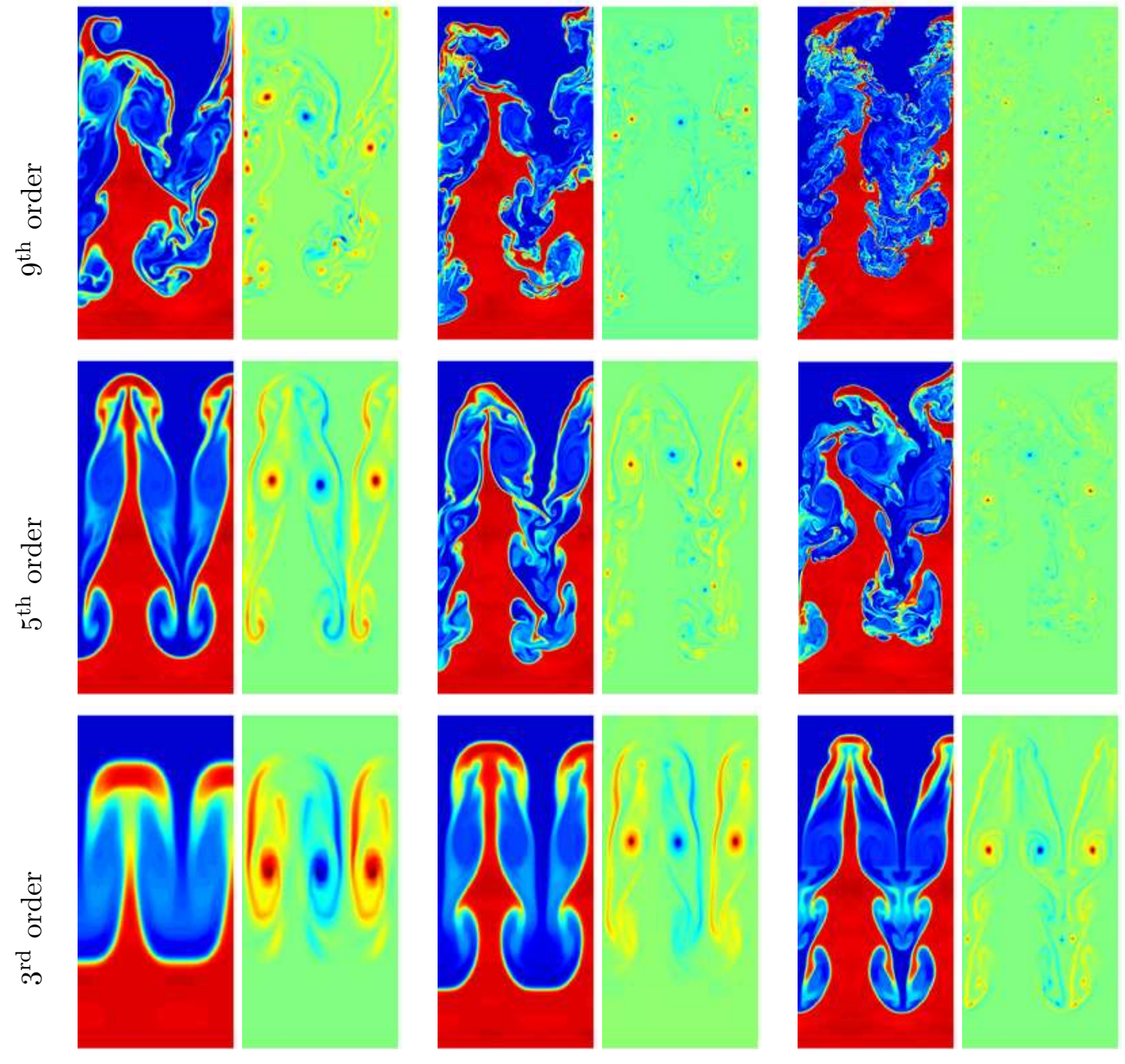

Figure 64: Density and vorticity at $t=12 \mathrm{~ms}$. 

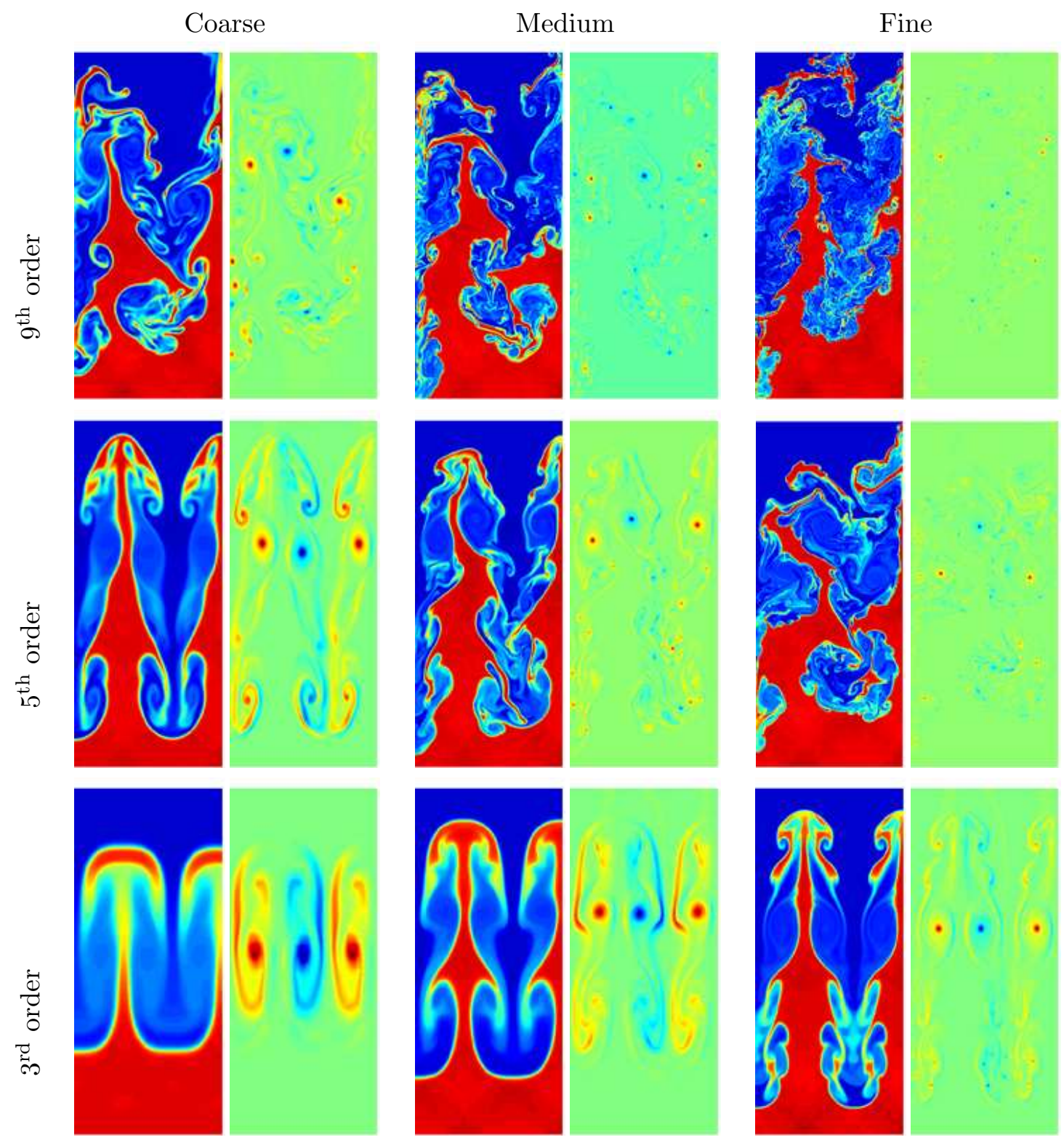

Figure 65: Density and vorticity at $t=13 \mathrm{~ms}$. 

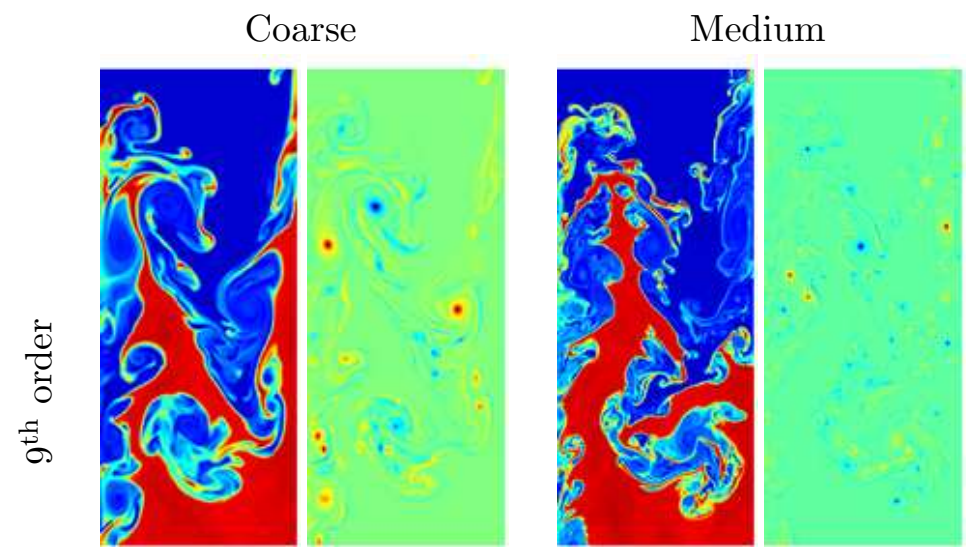

Fine
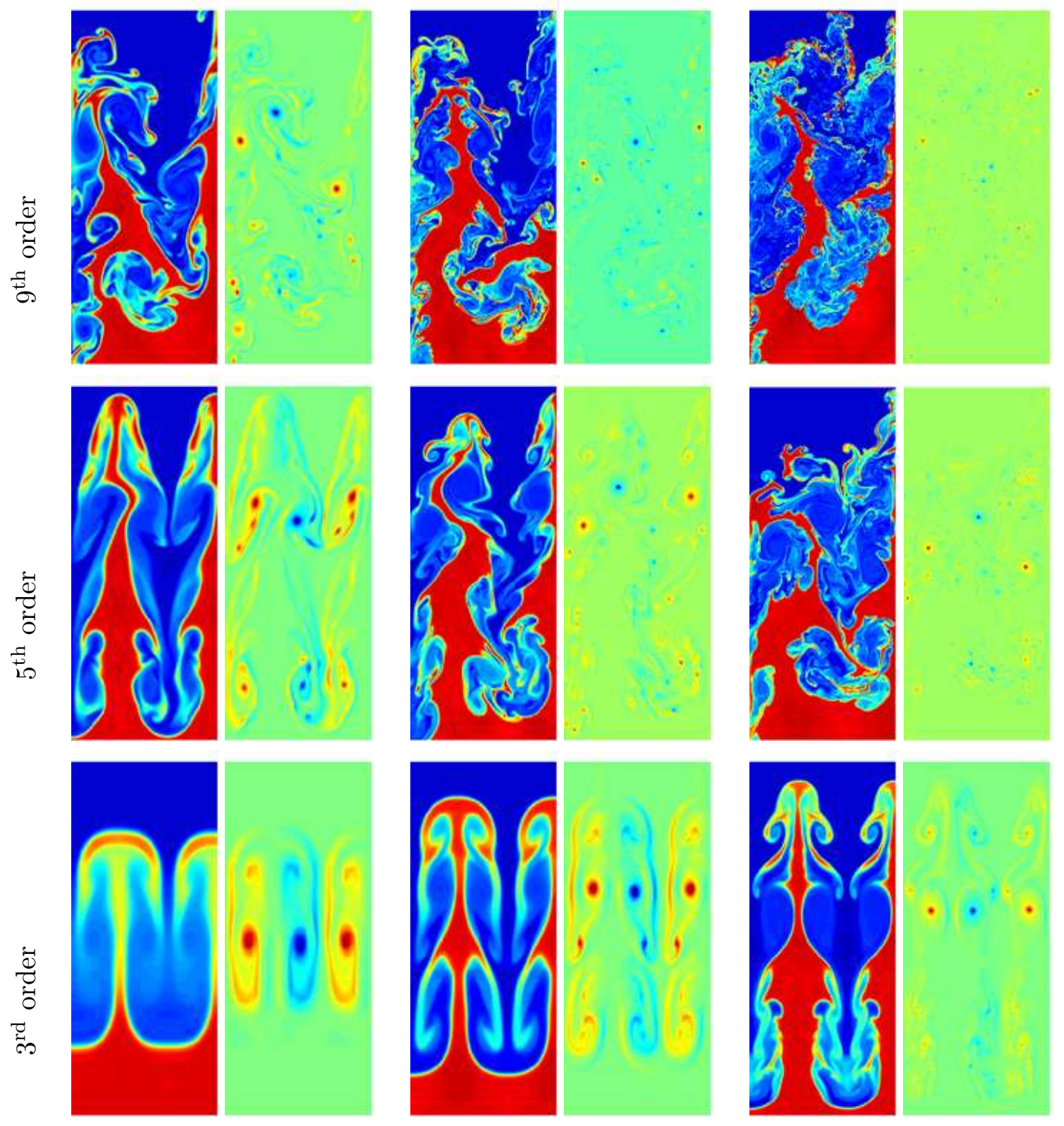

Figure 66: Density and vorticity at $t=14 \mathrm{~ms}$. 

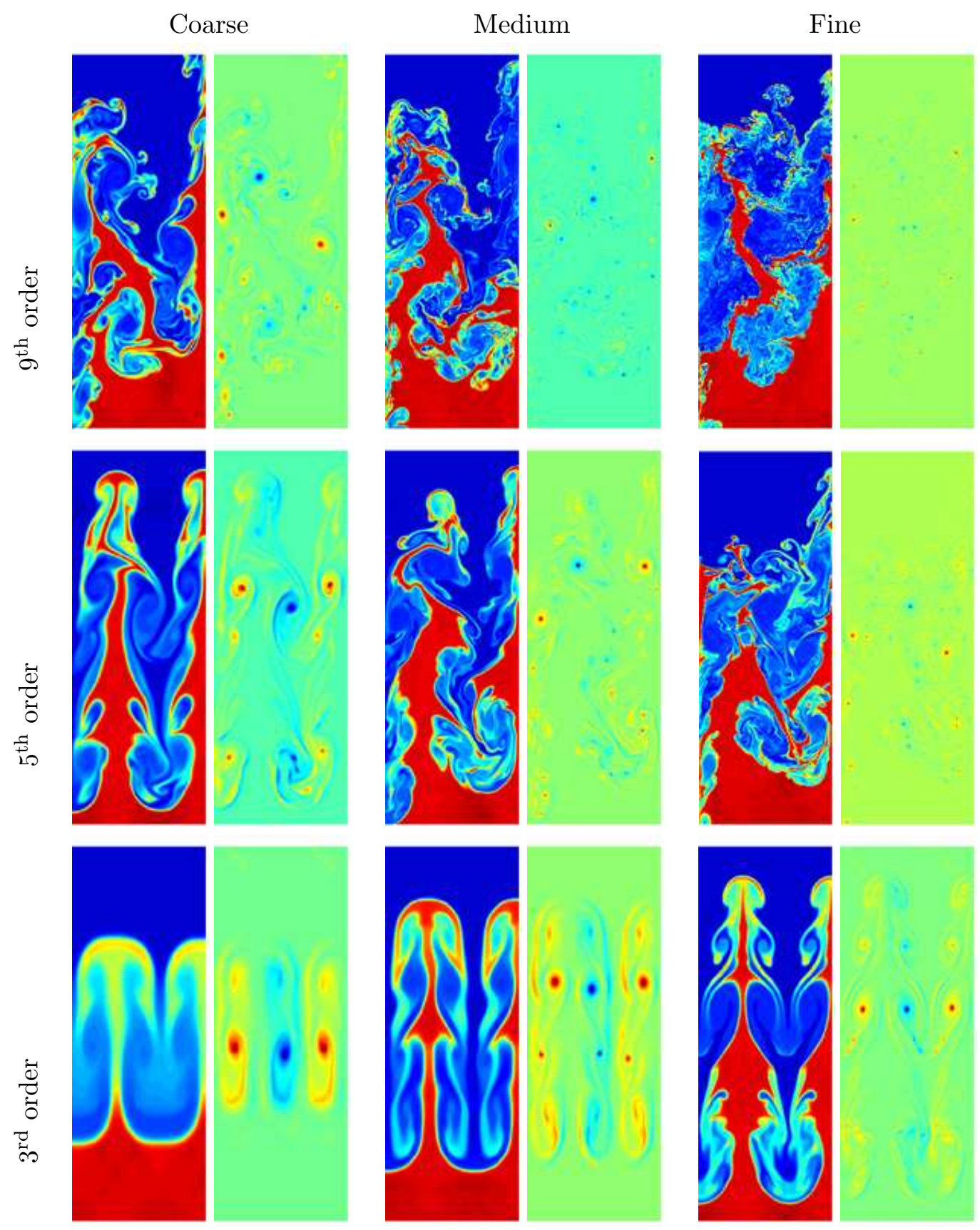

Figure 67: Density and vorticity at $t=15 \mathrm{~ms}$. 

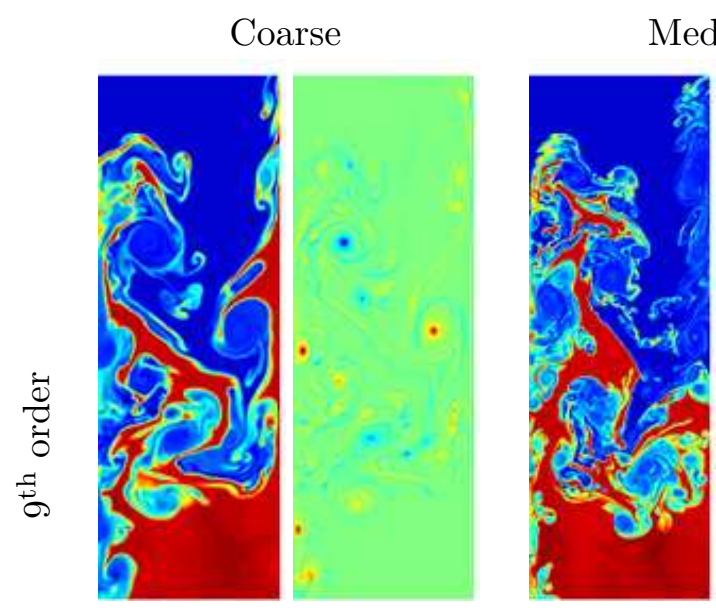

Fine
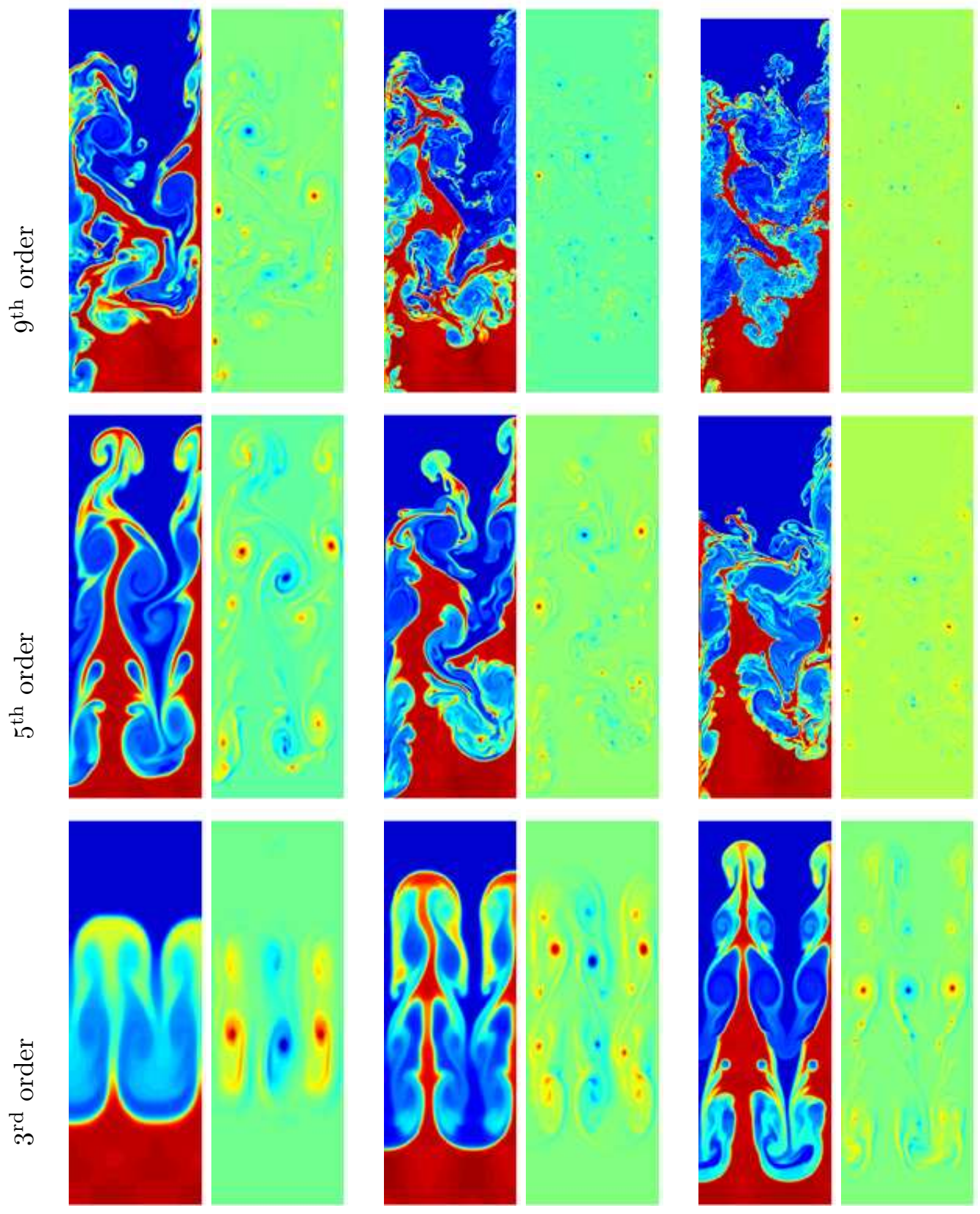

Figure 68: Density and vorticity at $t=16 \mathrm{~ms}$. 

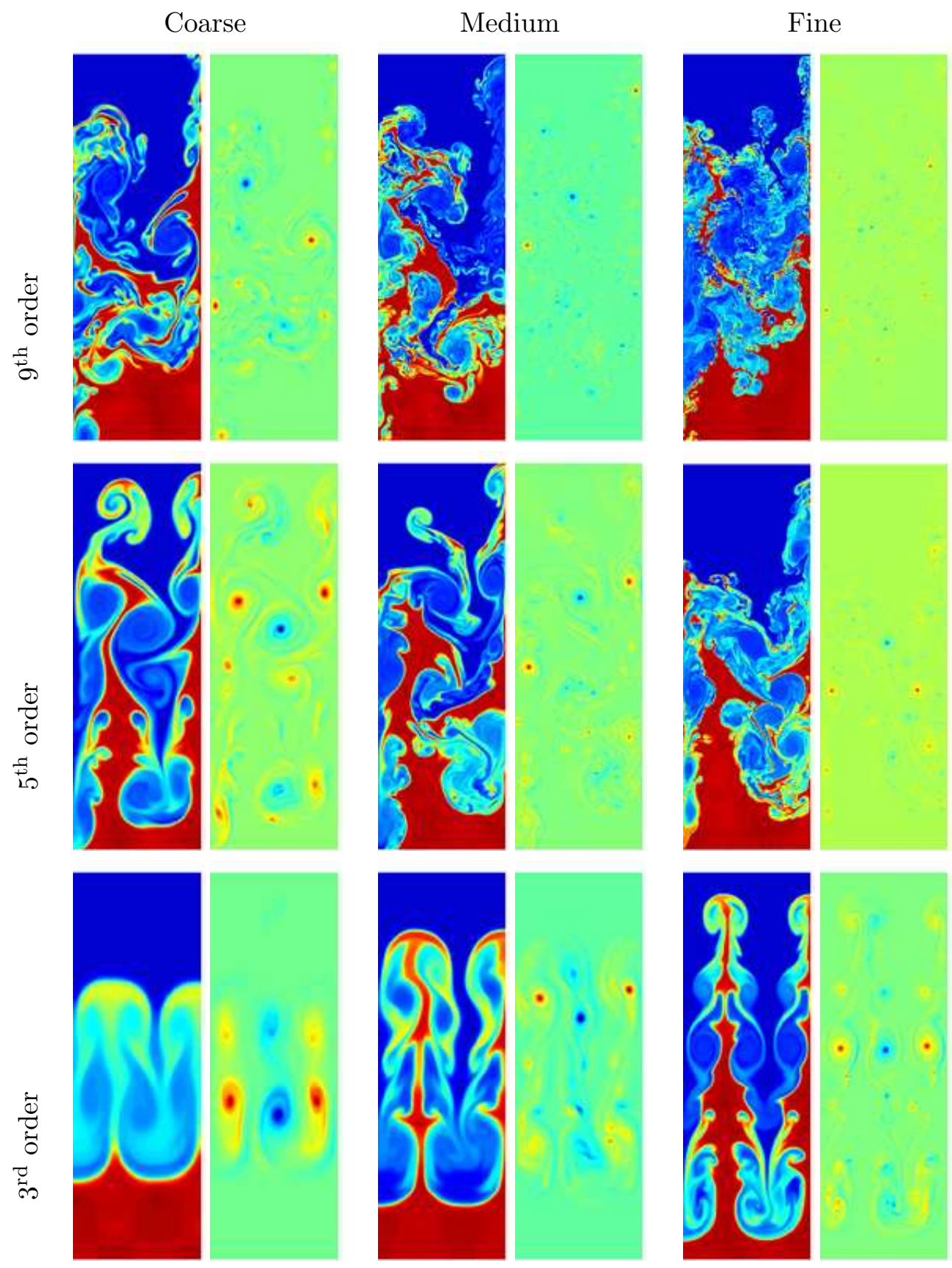

Figure 69: Density and vorticity at $t=17 \mathrm{~ms}$. 

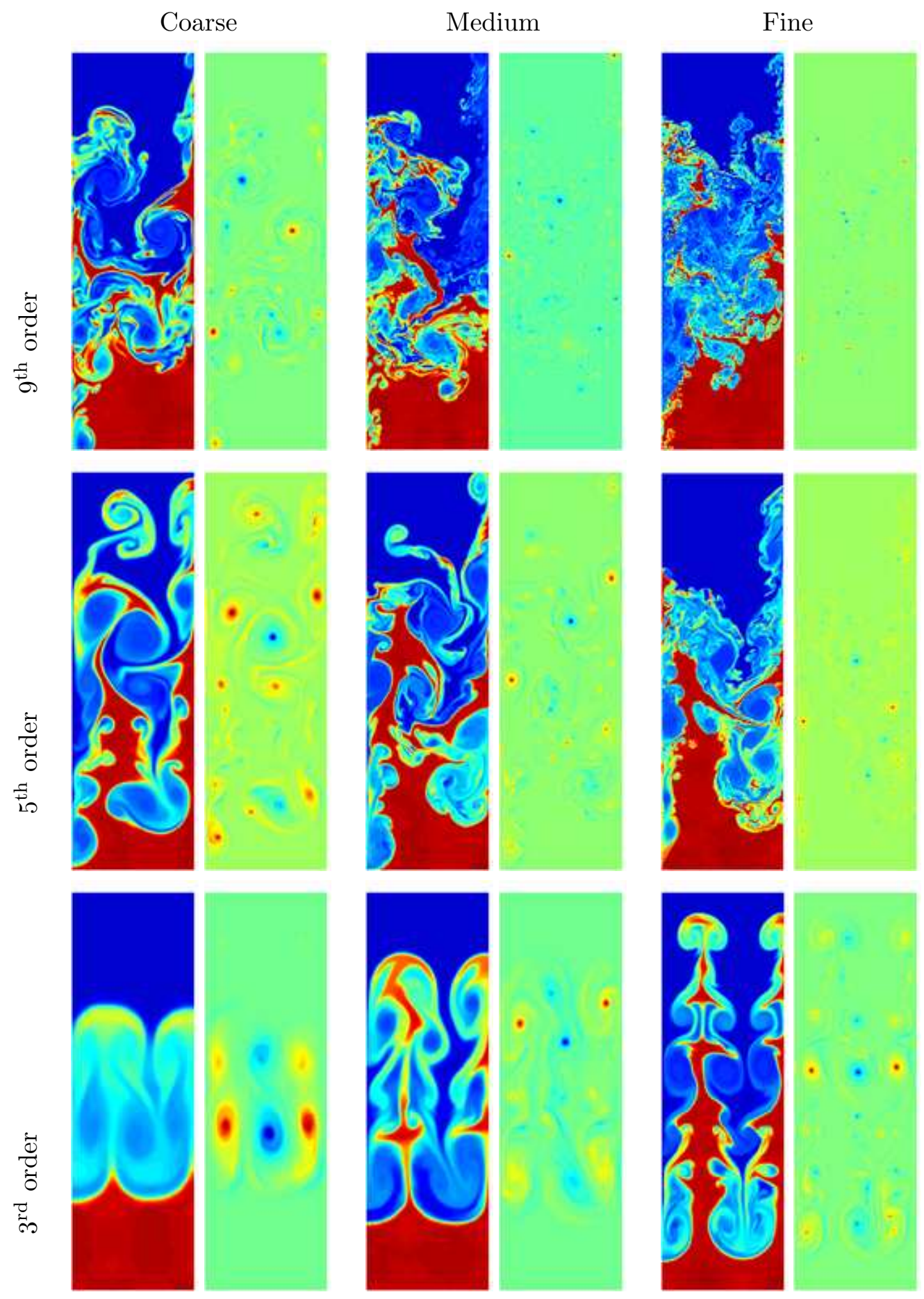

Figure 70: Density and vorticity at $t=18 \mathrm{~ms}$. 


\begin{tabular}{|c||c|c|c|}
\hline Order & Coarse resolution & Medium resolution & Fine resolution \\
\hline \hline 9 & 0.4 & 2.3 & 20.7 \\
5 & 0.2 & 1.0 & 9.5 \\
3 & 0.17 & 0.8 & 7.1 \\
\hline
\end{tabular}

Table 3: Ratio of CPU times for advancing the simulations by $\Delta t=0.1 \mathrm{~ms}$ compared with the time needed for the fifth-order simulation at medium resolution.

\subsection{Computational scaling of the simulations}

The simulations presented in this report were conducted on the Blue Pacific supercomputer at the Lawrence Livermore National Laboratory. For each case, 32 nodes were used with a total of 128 processors. Each simulation was carried out to a time of at least $t=18 \mathrm{~ms}$. The CPU times required to advance the simulation between $t=12.5 \mathrm{~ms}$ and $t=12.6 \mathrm{~ms}$ was compared with the time required for the fifth-order medium grid simulation. The late times are chosen because at earlier times the domain increases due to the adaptive domain feature of the HOPE code [57]. The ratio of the CPU times for the simulations and the time needed for the fifth-order medium grid case are presented in Table 3. Increasing the order of WENO reconstruction requires twice the computational time, whereas doubling the grid resolution requires five to eight times more computational time.

The analysis presented in this report suggests that similar results are observed when either the order of reconstruction was increased, keeping the grid resolution fixed, or the resolution was doubled, keeping the order of reconstruction fixed. The CPU times suggest that the use of high-order methods is less computationally expensive than increasing the grid resolution. Thus, the use of high order methods can lead to a significant advantage in three-dimensional simulations of complex hydrodynamic flows. 


\section{Summary and conclusions}

The high-resolution weighted essentially non-oscillatory (WENO) shock-capturing method implemented in the HOPE code has been applied to investigate the classical two-dimensional, single-mode Richtmyer-Meshkov instability in planar geometry with reshock. The current simulations were conducted in the spirit of monotone-integrated large-eddy simulation (MILES), i.e., molecular dissipation and diffusion terms in the governing equations were neglected. The initial conditions and computational domain approximate the Mach 1.21 air(acetone)/SF 6 shock tube experiment of Collins and Jacobs [23]. Only the test section of the shock tube was simulated, so that the reflected rarefaction wave present in the experiment was not explicitly captured in the simulations. A single value of the adiabatic exponent was used, as additional algorithm development is required for the two-fluid implementation in the HOPE code. Future simulations with explicit molecular dissipation and with subgrid-scale models are also planned to more accurately model the flow physics. Simulations with eleventh-order reconstruction are also envisaged.

The simulations were performed in two spatial dimensions using the Euler equations for the following reasons:

1. the initial conditions based on the Collins and Jacobs shock tube experiment are effectively two-dimensional, so that a two-dimensional simulation is expected to capture the essential flow features prior to reshock;

2. it is possible to achieve much higher spatial resolution in two dimensions than in three dimensions, thereby affording highly-resolved simulations that are less affected by numerical diffusion;

3. two-dimensional simulations allow the study of the breakup of structures and the transfer of energy to small scales in the absence of the vortex stretching mechanism, and facilitate comparisons to previous two-dimensional simulations;

4. it is computationally challenging to perform three-dimensional simulations with explicit molecular dissipation and diffusion that capture all of the spatio-temporal scales present in a complex flow, particularly following reshock, while sharply capturing the shock.

The complex issues concerning the consequences of the reduction of the accuracy of numerical solutions of nonlinear problems to first-order upon shock passage $[65,18,19]$ are not addressed in the present work. In this sense, the spatial truncation error of a higher-order method can be parameterized in terms of the grid resolution $\Delta x$ by

$$
\varepsilon(r, \Delta x)=c \Delta x+c_{r}(\Delta x)^{r},
$$

where $c$ and $c_{r}$ are constants dependent upon the flow and on the specific numerical scheme, and $r$ is the spatial order of accuracy of the scheme $(n . b$. , the error increases with increasing shock strength). The first term on the right side is the error due to the shock, and the second term on the right is the error in a smooth flow region. Note that only those regions in the computational domain where information propagates through a shock are susceptible to such shock-capturing errors. While these issues are common to all shock-capturing methods (irrespective of their formal order of accuracy) when the initial shock passes through a perturbed interface to generate the Richtmyer-Meshkov instability and when the reshock of the evolving 
interface occurs, it is quite apparent from the present investigation that the properties of the relatively smooth and nearly-incompressible flow following reshock is strongly affected by the order of the method. This topic deserves further investigation and analysis.

Numerical simulations were performed using third-, fifth-, and ninth-order spatial flux reconstruction and uniform spatial grid resolutions with 128, 256, and 512 points per initial perturbation wavelength. To our knowledge, the present work represents the first comprehensive application of the WENO method to the computational study of the classical RichtmyerMeshkov instability with reshock. It should be noted that a detailed numerical study of the single-mode, 'impulsive Richtmyer-Meshkov' instability experiment with reshock of Jacobs, Jones and Niederhaus [46, 78, 79] was performed by Kotelnikov and Zabusky [52] and Kotelnikov, Ray and Zabusky [51] using the vortex-in-cell method and the contour advection semiLagrangian method (n.b., Kotelnikov, Ray and Zabusky [51] also simulated the Jacobs et al. [45] and Rightley et al. [88] Mach 1.2 experiment with reshock using a Godunov method, and compared the results with their incompressible results). Furthermore, to our knowledge, the present work is the first systematic investigation of the effects of both order of reconstruction and grid resolution in a given high-resolution method, as applied to this instability. The comparison of results obtained with different grid resolutions and reconstructions is similar in spirit to the work of Cook, Cabot and Greenough [24], in which a centered ENO scheme, a higher-order Godunov method, and a filtered spectral/compact difference method (applied to the Shu-Osher problem, Collins-Jacobs experiment prior to reshock, and Taylor-Green vortex) were compared. The comparison of results obtained with fifth- and ninth-order WENO reconstruction in the present study showed that similar structures can be obtained using the higher-order reconstruction with one-half of the grid resolution in each direction - a similar conclusion obtained previously for the Rayleigh-Taylor instability [93]. This result can be explained using (202) as follows. The error can be decreased by halving $\Delta x$ or by doubling $r$. Let $\varepsilon(r, \Delta x)$ in Eq. (202) represent the error for a given $\Delta x$ and $r$. Let $\Delta x^{\prime}=\Delta x / 2$ and $r^{\prime}=2 r$ (corresponding to doubling the grid resolution and doubling the order). Doubling the grid resolution gives

$$
\begin{aligned}
\varepsilon\left(r, \Delta x^{\prime}\right) & =c \Delta x^{\prime}+c_{r}\left(\Delta x^{\prime}\right)^{r} \\
& =c \frac{\Delta x}{2}+c_{r}(\Delta x)^{r}\left(\frac{1}{2}\right)^{r},
\end{aligned}
$$

while doubling the order of spatial reconstruction gives

$$
\begin{aligned}
\varepsilon\left(r^{\prime}, \Delta x\right) & =c \Delta x+c_{r^{\prime}}(\Delta x)^{r^{\prime}} \\
& =c \Delta x+c_{r^{\prime}}(\Delta x)^{r}(\Delta x)^{r} .
\end{aligned}
$$

If $c_{r^{\prime}} \sim c_{r}$ then, comparing the truncation errors in Eqs. (203) and (204) reveals that doubling the order of reconstruction yields an overall smaller truncation error in the smooth region of the flow as $\Delta x<1 / 2$. Since much of the instability evolution occurs in regions far away from the shock, this explains why doubling the order of reconstruction gives comparable results to doubling the grid resolution. Note that near the shock, doubling the grid resolution results in a smaller error than doubling the order of reconstruction. This reflects the trade-off between the formal design order of a scheme and spatial resolution, which becomes even more computationally significant in three spatial dimensions.

While the numerical simulations presented here have several limitations (including the consideration of only the shock tube test section, and two-dimensional flow), the excellent agreement with experimental data prior to reshock provides encouragement for the continued use of 
the WENO method for the quantitative investigation of complex hydrodynamic flows induced by shocks. In particular, Part 3 [58] and Part 4 [59] of this report will consider the oblique single-mode Richtmyer-Meshkov instability in two dimensions and the multi-mode RichtmyerMeshkov instability with reshock in two and three dimensions, respectively. Presented below is a summary of the key findings in each of the three principal subjects of investigation.

\subsection{Mixing layer growth}

A comprehensive analysis of the instability evolution was presented in the first part of the report in $\S 2$ and 3, including: (1) a comparison of the density from a fifth- and ninth-order WENO simulation (using a uniform grid resolution of 256 points per initial perturbation wavelength) to experimental PLIF images, and; (2) a comparison of the mixing layer growth before and after reshock with the predictions of analytical, semi-analytical, and phenomenological amplitude (mixing layer) growth models. The simulations were conducted up to a time $t=18 \mathrm{~ms}$, much later than reported in the Collins and Jacobs [23] experiment $(t=11 \mathrm{~ms})$.

\subsubsection{Comparison to linear and nonlinear models prior to reshock}

The amplitude growth prior to reshock was compared to the experimental data and to the predictions of various classical and recent models for amplitude growth in the linear and weakly-nonlinear regimes. Excellent quantitative agreement was found between the amplitude obtained from the fifth-order simulation on the medium resolution ( 256 points per initial perturbation wavelength) grid and the experimental data points prior to reshock. The density fields obtained from the simulation were also in excellent qualitative agreement with the corrected density PLIF images from the experiment prior to reshock. As expected, the simulation data was also in excellent agreement with linear models at early times $t$ satisfying $k v_{0} t \lesssim 1$, where $v_{0}$ is given in Eq. (12). The simulation data was also found to be in best agreement with the predictions of the Zhang-Sohn Padé amplitude growth model. Thus, this component of the present work serves as further validation of the HOPE code and of the WENO method against experimental data.

\subsubsection{Comparison to reshock models}

Following reshock, it was shown that the instability evolution did not agree with the experimental PLIF images, as the rarefaction wave present in the experiment was not captured in the numerical simulation. As a result, the density fields obtained from the simulation and the corrected density PLIF images from the experiment showed a time lag of $\approx 1 \mathrm{~ms}$. Simulated density Schlieren images were also presented to illustrate the complex wave interactions occurring during the reshock process, i.e., the reflected and transmitted waves, and the focusing effects that are difficult to image experimentally. The growth of the mixing layer following reshock obtained from the numerical simulation was compared to the predictions of the Mikaelian [71] and Brouillette-Sturtevant [15] post-reshock amplitude growth models. The simulation data was found to be in very good agreement with the linear-in-time growth predicted by the Mikaelian model immediately following reshock. The Brouillette-Sturtevant model also predicts a linear growth, but with a smaller slope than the Mikaelian model. At later times following reshock, the simulation data lies between the predictions of the Mikaelian and Brouillette-Sturtevant models. As also expected, the amplitudes turn over and grow at 
a slower rate at sufficiently large times following reshock. In general, it is clear that additional experimental data is needed to provide a more complete validation, especially following reshock.

\subsubsection{The effects of outflow and reflecting boundary conditions}

The effects of reflecting or outflow boundary conditions at the shock tube end wall (end of the computational domain) on the amplitude growth and other properties were investigated. In particular, it was shown that the mixing layer width computed using the reflecting boundary condition was significantly larger than that computed using the outflow boundary condition following the arrival of the reflected rarefaction wave at $t \approx 10 \mathrm{~ms}$. The widths differed by $\approx 9 \mathrm{~cm}$ at a time $8 \mathrm{~ms}$ following the arrival of the reflected rarefaction wave. As the outflow boundary condition case eliminates wave-interface interactions subsequent to reshock, the flow transitions to a decaying flow at sufficiently large times after reshock. A quasi-decaying flow develops at late times in the reflecting boundary condition case, as multiple (successively weaker) wave-interface interactions occur. To our knowledge, this is the first numerical study that removed the effects of reflected waves on the evolution after reshock, and showed their important role on the dynamics of the flow following reshock.

\subsection{Local and global mixing analysis}

The second part of the report in $\S 4$ considered an extensive investigation of mixing before and after reshock. Mole fractions, fast kinetic models, and fluctuating energy spectra were used to characterize the mixing in the direction of propagation of the shock. Mixing fractions and statistics were also considered to characterize the overall mixing and distribution of energy as a function of time.

\subsubsection{Profiles, mixing fractions, spectra, and statistics}

The analysis included an examination of the profiles of mole fractions and other quantities characterizing the mixing over the extent of the layer as a function of time. Many of these quantities were adopted from previous analysis of Rayleigh-Taylor mixing, suitably modified for their application to shocked compressible flow. The profiles were recentered by the location of the midpoint of the interface and rescaled by the total mixing layer width in order to investigate the dynamics of mixing within the layer and the apparent collapse of the profiles at late times. The observed characteristics of the mixing averaged over the direction of shock propagation were qualitatively explained using the product mole fraction profiles, volume fraction profiles, and other related quantities.

The time-evolution of the one-dimensional Fourier spectra of the fluctuating kinetic energy components, enstrophy, density variance, and pressure variance was also studied. It was shown that the kinetic energy spectrum decays following reshock and at late times during the quasi-decay phase. The streamwise component of the kinetic energy spectrum dominates the spanwise component at all times, indicating that spectral isotropy is not achieved. The fluctuating enstrophy spectrum shows significant energy content in the intermediate and small-scales. The density variance spectrum shows little sensitivity to reshock. By contrast, the pressure variance is highly sensitive to reshock and to the interactions with the reflected waves. To our knowledge, this represents the first examination of the fluctuation enstrophy, density variance and pressure variance spectra in a reshocked Richtmyer-Meshkov mixing layer. 
An apparent inertial range was observed for the density and pressure variance spectra over approximately a decade in wavenumber at late times following reshock. However, spatial resolutions with more than 512 points per initial perturbation wavelength are needed to support a wider range of spatial scales, in order to conclusively determine whether any scaling behavior of the one-dimensional spectra exists.

The time-evolution of global statistics (i.e., wavenumber-integrated spectra), as well as of volume-averaged quantities, was also studied. The decay characteristics following reshock for these quantities were exhibited. The approach to statistical isotropy of the streamwise and spanwise velocity fluctuations at late times was demonstrated. The fluctuating enstrophy displays decay at late times following the interaction with the reflected rarefaction wave at $t \approx 10 \mathrm{~ms}$. The pressure variance showed the most sensitivity to the interaction of reflected waves with the mixing layer. By contrast, the density variance displayed the least sensitivity. These observations are consistent with those pertaining to the density and pressure variance spectra.

\subsubsection{The effects of reshock}

A detailed examination of the effects of reshock on the behavior of mixing profiles, mixing fractions, spectra, and statistics was presented. Several measures of enhanced mixing following reshock were discussed, as well as the amplification of fluctuations reflected in the energy spectra and statistics. It was shown that reshock imparts energy into all scales of the flow, with the amplification varying strongly with wavenumber. In general, the largest and intermediate scales showed the most energy amplification, while the smallest scales showed the least energy amplification. In the case of the kinetic energy fluctuations, the amplification was a constant over a wide range of intermediate and large wavenumbers. It was found that different quantities exhibit different sensitivities following reshock. Pressure fluctuations, as reflected in their spectra, increase strongly. Thus, quantities depending on pressure fluctuations cannot be neglected in turbulent transport and mixing models. By contrast, density fluctuations do not exhibit such strong sensitivity to reshock.

\subsubsection{The effects of outflow and reflecting boundary conditions}

The effects of outflow and reflecting boundary conditions at the end wall of the shock tube test section on mixing quantities, spectra and statistics was also considered. Mixing fractions obtained with the outflow boundary condition were larger than those obtained with the reflecting boundary condition, as the molar fractions in the former were computed over a smaller mixing layer width. By contrast, the statistics obtained with the outflow boundary condition yielded larger values than those obtained with the reflecting boundary condition, as the interaction with the reflected rarefaction wave in the former further excited the fluctuations. In addition, statistical isotropy is not achieved with the outflow boundary condition, as the streamwise and spanwise fluctuating kinetic energy maintain a constant separation in time; wavenumber-integrated spectra (global statistics) also exhibited differences. This comparison further supports the important role that reflected waves have in the dynamics of the flow following reshock. These results also have important implications for modeling turbulent transport and mixing induced by the Richtmyer-Meshkov instability with reshock. 


\subsection{The effects of order of reconstruction and grid resolution}

A comprehensive investigation of the effects of order of reconstruction and grid resolution on the mixing layer growth, mole fractions, spectra, spatially-averaged mixing fractions, and statistics was presented in the third part of the report in $\S 5$ and 5.7. Prior to reshock, these quantities exhibit relatively little sensitivity to the reconstruction order and grid resolution. However, they exhibited much more sensitivity to these numerical parameters following reshock, including in the late-time, quasi-decay phase. Following reshock, the mixing layer width exhibited increased sensitivity to the order of spatial reconstruction and grid resolution. The growth rates obtained from the high-order and high-resolution simulations were larger than those obtained from lower order and lower resolution simulations. This is due to the different amounts of numerical viscosity associated with each such simulation. High-resolution, high-order methods have smaller amounts of intrinsic numerical diffusion. Large numerical diffusion prevents the formation of small-scale features that are responsible for increased growth of the mixing layer following reshock.

The expression (202) suggests that the error of a high-order method vanishes in the limit $\Delta x \downarrow 0$. However, in general, there are no theoretical proofs of the convergence of multidimensional numerical solutions obtained using nonlinear methods for general flows described by the non-dissipative, compressible fluid dynamics equations (i.e., Euler equations) [55]. Hence, there is no guarantee of pointwise convergence of the numerical solution, i.e., grid refinement and increasing the order of resolution is not necessarily expected to yield pointwiseconverged quantities. However, 'convergence' can be observed in large-scale quantities, such as the mixing layer width. In fact, as the order of reconstruction and the grid is refined, the mixing layer width prior to reshock also appears to 'converge'. It is interesting to note that some 'convergence' is also observed for the width following reshock ( $t \geq 6 \mathrm{~ms}$ ), but prior to the arrival of the reflected rarefaction wave at $t \approx 10 \mathrm{~ms}$. As the grid is refined and the order of reconstruction is increased, the discrepancies between the widths decrease and the width obtained from the ninth-order simulation on the medium grid is nearly the same as that obtained from the ninth-order simulation on the fine grid. This 'convergence' is no longer apparent after the arrival of the reflected rarefaction wave, as the difference between the widths increases with time. The late-time width shows significant variation as the order of reconstruction is increased and the grid is refined, indicating that parameterizations of apparent power-law growth at late times using general expressions of the form

$$
h(t)=a+b\left(t+t_{0}\right)^{\theta}
$$

where $a$ and $b$ are parameters, and $t_{0}$ is a virtual time origin [43], must be performed with caution. An analysis of the spectra from the simulations with different orders and resolutions compared to the spectrum obtained from the ninth-order simulation on the fine grid showed that there is no apparent 'convergence' of these spectra. This, together with an analysis of mixing fractions and other quantities sensitive to molecular mixing, shows that quantities sensitive to the small-scale structure of the flow do not generally exhibit 'convergence', while quantities determined primarily by the large-scale structures appear to be 'converged' at sufficiently high order of reconstruction and grid resolution. Hence, large-scale (e.g., amplitude) data is insufficient to validate numerical simulation results, as quantities sensitive to fluctuations can differ significantly, while the large-scale structures are insensitive. These issues, and additional quantities such as probability distributions, other statistics, and turbulent transport quantities, will be discussed further in Part 4 of this report [59]. Difference norms and other quantitative 
measures of the difference between simulation data of different order and resolution is relegated to future work.

Statistics and spectra that had significant contributions from intermediate- and small-scale structures exhibited the most sensitivity as the order of reconstruction was increased and the grid was refined. The third-order simulations exhibited very large numerical diffusion, even at the highest resolution of 512 points per initial perturbation wavelength. This results in the preservation of large-scale structures as the fluctuations associated with small-scale structures are strongly damped. As a result, well-defined, nearly-symmetrical, large-scale structures persisted to late times following reshock. This is reflected in the mole fractions, mixing fractions, spectra, and statistics from the third-order simulations, differing significantly from the results from the higher order simulations. Thus, the use of data from third-order WENO simulations at these spatial resolutions to quantify mixing is not well-justified. By contrast, simulations with increasing order of spatial reconstruction and increasing spatial resolution exhibited the development of asymmetry and fragmentation of large structures at late times following reshock, resulting in increased mixing. This is reflected in the mole fractions that exhibit strongly-oscillatory behavior at late times.

The variation of the order of reconstruction and grid resolution in the present study investigated only the spatial discretization error in the method. It would be of interest to investigate the error in the temporal discretization by comparing the present results obtained using the third-order TVD Runge-Kutta scheme to those obtained using a second- and fourth-order time-evolution scheme, to investigate the nature of the cumulative errors.

\subsection{Conclusions}

Very little experimental data is available after reshock to aid in validating and constraining numerical simulation data. The present simulations provide pointwise data that can be used to compute profiles across the mixing layer, measures of mixing, spectra, and statistics. Thus, this work is representative of an effort to use a validated numerical method to obtain detailed data not available from experiments. For example, there are currently no experimental measurements of molecular mixing or data concerning the structure of the mixing layer. Also, energy spectra have not been obtained from experimental data. Experimental data accurately measuring quantities depending on spatial derivatives or quantities sensitive to small-scale mixing (e.g., mixing fractions) would be very helpful in discriminating between the different simulation results obtained across a range of orders and resolutions.

The quantities investigated in this report are not exhaustive. Additional quantities will be considered in Part 4 of this report [59], including probability distribution functions and turbulent transport quantities. Detailed data from such simulations can be used to investigate turbulence model initialization, as well as the properties and predictions of turbulent transport and mixing models following reshock and late in time.

\section{Acknowledgments}

The authors thank Dr. Wai-Sun Don from Brown university for assistance with all aspects of the HOPE code. Marco Latini also acknowledges support from the Air Force Office of Scientific Research through the National Defense Science and Engineering Graduate Fellowship. This work was performed under the auspices of the U.S. Department of Energy by the University of California, Lawrence Livermore National Laboratory under Contract No. W-7405-Eng-48. 


\section{References}

[1] U. Alon, J. Hecht, D. Mukamel, and D. Shvarts, Scale invariant mixing rates of hydrodynamically unstable interfaces, Phys. Rev. Lett. 72 (1994), 2867-2870.

[2] U. Alon, J. Hecht, D. Ofer, and D. Shvarts, Power laws and similarity of Rayleigh-Taylor and Richtmyer-Meshkov mixing fronts at all density ratios, Phys. Rev. Lett. 74 (1995), $534-537$.

[3] U. Alon, D. Shvarts, and D. Mukamel, Scale-invariant regime in Rayleigh-Taylor bubblefront dynamics, Phys. Rev. E 48 (1993), 1008-1014.

[4] M. J. Andrews and D. B. Spalding, A simple experiment to investigate two-dimensional mixing by Rayleigh-Taylor instability, Phys. Fluids A 2 (1990), 922-927.

[5] D. Arnett, The role of mixing in astrophysics, Astrophys. J. Suppl. 127 (2000), 213-217.

[6] W. D. Arnett, Supernova theory and supernova 1987A, Astrophys. J. 319 (1987), 136142.

[7] W. D. Arnett, B. A. Fryxell, and E. Müller, Instabilities and nonradial motion in SN1987A, Astrophys. J. 341 (1989), L63-L66.

[8] S. Atzeni and J. Meyer-ter Vehn, The Physics of Inertial Confinement Fusion: Beam Plasma Interaction, Hydrodynamics, Hot Dense Matter, International Series of Monographcs on Physics, vol. 125, Oxford University Press, New York, 2004.

[9] D. Balsara and C.-W Shu, Monotonicity preserving weighted essentially non-oscillatory schemes with increasingly high order of accuracy, J. Comput. Phys. 160 (2000), 405-452.

[10] G. I. Barenblatt, Nonlinear Dynamics and Turbulence, Pitman, Boston, 1983.

[11] C. M. Bender and S. A. Orszag, Advanced Mathematical Methods for Scientists and Engineers, Springer-Verlag, New York, 1999.

[12] J. P. Boris, F. F. Grinstein, E. S. Oran, and R. L. Kolbe, New insights into large eddy simulation, Fluid Dyn. Res. 10 (1992), 199-228.

[13] M. Brouillette, The Richtmyer-Meshkov instability, Ann. Rev. Fluid Mech. 34 (2002), 445-468.

[14] M. Brouillette and B. Sturtevant, Growth induced by multiple shock waves normally incident on plane gaseous interfaces, Physica D 37 (1989), 248-263.

[15] _ Experiments on the Richtmyer-Meshkov instability: single-scale perturbations on a continuous interface, J. Fluid Mech. 263 (1994), 271-292.

[16] R. L. Burden and J. D. Faires, Numerical Analysis, Brooks/Cole Publishing Company, Pacific Grove, CA, 1997.

[17] C. Canuto, M. Y. Hussaini, A. Quarteroni, and T. A. Zang, Spectral Methods in Fluid Dynamics, Springer-Verlag, New York, 1990. 
[18] M. H. Carpenter and J. H. Casper, Computational considerations for the simulation of discontinuous flows, Barriers and Challenges in Computational Fluid Dynamics (V. Venkatakrishnan, M. D. Salas, and S. R. Chakravarthy, eds.), Kluwer Academic, Dordrecht, 1998, pp. 63-78.

[19] _ Accuracy of shock capturing in two spatial dimensions, AIAA J. 37 (1999), 1072-1079.

[20] S. Chandrasekhar, Hydrodynamic and Hydromagnetic Stability, Dover Publications, New York, 1961.

[21] A. J. Chorin and J. E. Marsden, A Mathematical Introduction to Fluid Mechanics, third ed., Texts in Applied Mathematics, vol. 4, Springer-Verlag, New York, 1993.

[22] R. H. Cohen, W. P. Dannevik, A. M. Dimits, D. E. Eliason, A. A. Mirin, Y. Zhou, D. H. Porter, and P. R. Woodward, Three-dimensional simulation of a Richtmyer-Meshkov instability with a two-scale initial perturbation, Phys. Fluids 14 (2002), 3692-3709.

[23] B. D. Collins and J. W. Jacobs, PLIF flow visualization and measurements of the Richtmyer-Meshkov instability of an air/SF $F_{6}$ interface, J. Fluid Mech. 464 (2002), 113136.

[24] A. W. Cook, W. H. Cabot, and J. A. Greenough, A High-Order Method for Shock-Induced Mixing, Tech. Report UCRL-JC-144109, Lawrence Livermore National Laboratory, 2001.

[25] A. W. Cook and P. E. Dimotakis, Transition stages of Rayleigh-Taylor instability between miscible fluids, J. Fluid Mech. 443 (2001), 69-99, Corrigendum, J. Fluid Mech. vol 457, pg 410-411, 2002.

[26] G. Dimonte, D. L. Youngs, A. Dimits, S. Weber, M. Marinak, S. Wunsch, C. Garasi, A. Robinson, M. J. Andrews, P. Ramaprabhu, A. C. Calder, B. Fryxell, J. Biello, L. Dursi, P. MacNeice, K. Olson, P. Ricker, R. Rosner, F. Timmes, H. Tufo, Y.-N. Young, and M. Zingale, A comparative study of the turbulent Rayleigh-Taylor instability using high-resolution three-dimensional numerical simulations: The Alpha-Group collaboration, Phys. Fluids 16 (2004), 1668-1693.

[27] W.-S. Don, 2004, Private communication.

[28] P. G. Drazin, Kelvin-Helmholtz instability of finite amplitude, J. Fluid Mech. 42 (1970), 321-335.

[29] P. G. Drazin and W. H. Reid, Hydrodynamic Stability, second ed., Cambridge Monographs on Mechanics and Applied Mathematics, Cambridge University Press, Cambridge, 2003.

[30] D. Drikakis, Embedded turbulence model in numerical methods for hyperbolic conservation laws, Int. J. Num. Meth. Fluids 39 (2002), 763-781.

[31] _ Advances in turbulent flow computations using high-resolution methods, Prog. Aero. Sci. 39 (2003), 405-424. 
[32] R. E. Duff, F. H. Harlow, and C. W. Hirt, Effects of diffusion on interface instability between gases, Phys. Fluids 5 (1962), 417-425.

[33] S. W. Falk and W. D. Arnett, A theoretical model for type II supernovae, Astrophys. J. 180 (1973), L65-L68.

[34] G. Fraley, Rayleigh-Taylor stability for a normal shockwave-density discontinuity interaction, Phys. Fluids 29 (1986), 376-386.

[35] V. N. Goncharov, Analytical model of nonlinear, single-mode, classical Rayleigh-Taylor instability at arbitrary Atwood numbers, Phys. Rev. Lett. 88 (2002), 134501-1-134501-4.

[36] D. Gottlieb and S. A. Orszag, Numerical Analysis of Spectral Methods: Theory and Application, SIAM, Philadelphia, 1977.

[37] X. He, S. Chen, and R. Zhang, A lattice Boltzmann scheme for incompressible multiphase flow and its application in simulation of Rayleigh-Taylor instability, J. Comput. Phys. 152 (1999), 642-663.

[38] J. Hecht, U. Alon, and D. Shvarts, Potential flow models of Rayleigh-Taylor and Richtmyer-Meshkov bubble fronts, Phys. Fluids 6 (1994), 4019-30.

[39] L. F. Henderson, On the refraction of shock waves, J. Fluid Mech. 198 (1989), 365-386.

[40] D. J. Hill and D. I. Pullin, 2004, Private communication.

[41] R. L. Holmes, G. Dimonte, B. Fryxell, M. L. Gittings, J. W. Grove, M. Schneider, D. H. Sharp, A. L. Velikovich, R. P. Weaver, and Q. Zhang, Richtmyer-Meshkov instability growth: experiment, simulation and theory, J. Fluid Mech. 389 (1999), 55-77.

[42] R. L. Holmes, J. W. Grove, and D. H. Sharp, Numerical investigation of RichtmyerMeshkov instability using front-tracking, J. Fluid Mech. 301 (1995), 51-64.

[43] L. Houas and I. Chemouni, Experimental investigation of Richtmyer-Meshkov instability in shock tube, Phys. Fluids 8 (1996), 614-627.

[44] J. W. Jacobs, The dynamics of shock accelerated light and heavy gas cylinders, Phys. Fluids A 5 (1993), 2239-2247.

[45] J. W. Jacobs, D. G. Jenkins, D. L. Klein, and R. F. Benjamin, Nonlinear growth of the shock-accelerated instability of a thin fluid layer, J. Fluid Mech. 295 (1995), 23-42.

[46] J. W. Jacobs, M. A. Jones, and C. E. Niederhaus, Experimental studies of RichtmyerMeshkov instability, Proceedings of the Fifth International Workshop on Compressible Turbulent Mixing (R. Young, J. Glimm, and B. Boston, eds.), World Scientific, New York, 1996, pp. 195-202.

[47] J. W. Jacobs and J. M. Sheeley, Experimental study of incompressible Richtmyer-Meshkov instability, Phys. Fluids 8 (1996), 405-415.

[48] M. A. Jones and J. W. Jacobs, A membraneless experiment for the study of RichtmyerMeshkov instability of a shock-accelerated gas interface, Phys. Fluids 9 (1997), 3078-3085. 
[49] J. Kane, R. P. Drake, and B. A. Remington, An evaluation of the Richtmyer-Meshkov instability in supernova remnant formation, Astrophys. J. 511 (1999), 335-340.

[50] M. M. Koochesfahani and P. E. Dimotakis, Mixing and chemical reactions in a turbulent liquid mixing layer, J. Fluid Mech. 170 (1986), 83-112.

[51] A. D. Kotelnikov, J. Ray, and N. J. Zabusky, Vortex morphologies on reaccelerated interfaces: Visualization, quantification and modeling of one- and two-mode compressible and incompressible environments, Phys. Fluids 12 (2000), 3245-3264.

[52] A. D. Kotelnikov and N. J. Zabusky, Vortex dynamics of a twice-accelerated interface in an incompressible ideal fluid, Astrophys. J. Suppl. 127 (2000), 389-394.

[53] R. H. Kraichnan and D. Montgomery, Two-dimensional turbulence, Rep. Prog. Phys. 43 (1980), 547-619.

[54] K. Kremeyer, S. Nazarenko, and A. C. Newell, Shock bowing and vorticity dynamics during propagation into different transverse density profiles, Physica D 163 (2002), 150165.

[55] C. B. Laney, Computational Gasdynamics, Cambridge University Press, New York, 1998.

[56] M. Latini, Investigation of the Rayleigh-Taylor and Richtmyer-Meshkov Instabilities in Complex Geometries with Vortex Methods, In preparation.

[57] M. Latini and O. Schilling, Weighted Essentially Non-Oscillatory Simulations and Modeling of Complex Hydrodynamic Flows. Part 1. Regular Shock Refraction, Tech. Report UCRL-TR-205132, Lawrence Livermore National Laboratory, 2004.

[58] — Weighted Essentially Non-Oscillatory Simulations and Modeling of Complex Hydrodynamic Flows. Part 3. The Oblique Shock Richtmyer-Meshkov Instability, Tech. report, Lawrence Livermore National Laboratory, 2004.

[59] _ Weighted Essentially Non-Oscillatory Simulations and Modeling of Complex Hydrodynamic Flows. Part 4. Multi-Mode Richtmyer-Meshkov Instability with Reshock, Tech. report, Lawrence Livermore National Laboratory, 2004.

[60] D. Layzer, On the instability of superposed fluids in a gravitational field, Astrophys. J. 122 (1955), 1-12.

[61] M. Lesieur, C. Staquet, P. Le Roy, and P. Comte, The mixing layer and its coherence examined from the point of view of two-dimensional turbulence, J. Fluid Mech. 192 (1988), 511-534.

[62] R. J. Leveque, Finite Volume Methods for Hyperbolic Problems, Cambridge Texts in Applied Mathematics, Cambridge University Press, New York, 2002.

[63] J. D. Lindl, Development of the indirect-drive approach to inertial confinement fusion and the target physics basis for ignition and gain, Phys. Plasmas 2 (1995), 3933-4024.

[64] _ Inertial Confinement Fusion: The Quest for Ignition and Energy Gain Using Indirect Drive, AIP Press, New York, 1998. 
[65] A. Majda and S. Osher, Propagation of error into regions of smoothness for accurate difference approximations to hyperbolic equations, Comm. Pure Appl. Math. 30 (1977), $671-705$.

[66] A. Marquina and P. Mulet, A flux-split algorithm applied to conservative models for multicomponent compressible flows, J. Comput. Phys 185 (2003), 120-138.

[67] D. I. Meiron, G. R. Baker, and S. A. Orszag, Analytic structure of vortex sheet dynamics. Part 1. Kelvin-Helmholtz instability, J. Fluid Mech. 114 (1982), 283-298.

[68] R. Menikoff and C. Zemach, Rayleigh-Taylor instability and the use of conformal maps for ideal fluid flow, J. Comput. Phys 51 (1983), 28-64.

[69] E. E. Meshkov, Instability of the interface of two gases accelerated by a shock wave, Sov. Fluid Dyn. 4 (1969), 101-108.

[70] K. A. Meyer and P. J. Blewett, Numerical investigation of the stability of a shock accelerated interface between two fluids, Phys. Fluids 15 (1972), 753-759.

[71] K. O. Mikaelian, Turbulent mixing generated by Rayleigh-Taylor and Richtmyer-Meshkov instabilities, Physica D 36 (1989), 343-357.

[72] _ Freeze-out and the effect of compressibility in the Richtmyer-Meshkov instability, Phys. Fluids 1 (1994), 356-368.

[73] _ Numerical simulations of Richtmyer-Meshkov instabilities in finite-thickness fluid layers, Phys. Fluids 8 (1996), 1269-1292.

[74] _ Analytic approach to nonlinear Rayleigh-Taylor and Richtmyer-Meshkov instabilities, Phys. Rev. Lett. 80 (1998), 508-511.

[75] C. Mügler and S. Gauthier, Numerical simulations of single-mode Richtmyer-Meshkov experiments, Phys. Rev. E 58 (1998), 4548-4555.

[76] _ Two-dimensional Navier-Stokes simulations of gaseous mixtures induced by Richtmyer-Meshkov instability, Phys. Fluids 12 (2000), 1783-1798.

[77] S. Nakai and H. Takabe, Principles of inertial confinement fusion-physics of implosion and the concept of inertial fusion energy, Rep. Prog. Physics 59 (1996), 1071-1131.

[78] C. E. Niederhaus, Experiments on the Richtmyer-Meshkov Instability of Incompressible Fluids, Ph.D. thesis, University of Arizona, 2000.

[79] C. E. Niederhaus and J. W. Jacobs, Experimental study of the Richtmyer-Meshkov instability of incompressible fluids, J. Fluid Mech. 485 (2003), 243-277.

[80] Sadot O., L. Erez, U. Alon, D. Oron, and L. A. Levin, Study of nonlinear evolution of single-mode and two bubble interaction under Richtmyer-Meshkov instability, Phys. Rev. Lett. 80 (1998), 1654-1657.

[81] E. S. Oran and J. P. Boris, Numerical Simulation of Reactive Flow, second ed., Cambridge University Press, Cambridge, 2001. 
[82] G. Peng, N. J. Zabusky, and S. Zhang, Vortex-accelerated secondary baroclinic vorticity deposition and late-intermediate time dynamics of a two-dimensional Richtmyer-Meshkov interface, Phys. Fluids 15 (2003), 3730-3744.

[83] S. B. Pope, Turbulent Flows, Cambridge University Press, Cambridge, UK, 2000.

[84] J. K. Prasad, A. Rasheed, S. Kumar, and B. Sturtevant, The late-time development of the Richtmyer-Meshkov instability, Phys. Fluids 12 (2000), 2108-2115.

[85] J. Ray, Circulation Deposition and Interface Growth Rate in Richtmyer-Meshkov Environments: Scaling Laws and Reduced Models, Master's thesis, Rutgers University, 1997.

[86] K. I. Read, Experimental investigation of turbulent mixing by Rayleigh-Taylor instability, Physica D 12 (1984), 45-58.

[87] R. D. Richtmyer, Taylor instability in shock acceleration of compressible fluids, Comm. Pure Appl. Math. 8 (1960), 297-319.

[88] P. M. Rightley, P. Vorobieff, and R. F. Benjamin, Evolution of a shock-accelerated thin fluid layer, Phys. Fluids 9 (1997), 1770-1782.

[89] A. Rikanati, U. Alon, and D. Shvarts, Vortex model for the nonlinear evolution of the multimode Richtmyer-Meshkov instability at low Atwood numbers, Phys. Rev. E 58 (1998), $7410-7418$.

[90] O. Sadot, L. Erez, G. Oron, G. Ben-Dor, U. Alon, L. A. Levin, and D. Shvarts, Studies on the nonlinear evolution of the Richtmyer-Meshkov instability, Astrophys. J. Suppl. 127 (2000), 469-473.

[91] R. Samtaney, J. Ray, and N. J. Zabusky, Baroclinic circulation generation on shock accelerated slow/fast gas interfaces, Phys. Fluids 10 (1998), 1217-1230.

[92] R. Samtaney and N. J. Zabusky, Circulation deposition on shock-accelerated planar and curved density stratified interfaces: models and scaling laws, J. Fluid Mech. 269 (1994), $45-78$.

[93] J. Shi, Y.-T. Zhang, and C.-W. Shu, Resolution of high order WENO schemes for complicated flow structures, J. Comput. Phys 186 (2003), 690-696.

[94] S.-I. Sohn, Simple potential-flow model of Rayleigh-Taylor and Richtmyer-Meshkov instabilities for all density ratios, Phys. Rev. E 67 (2003), 026301-1-026301-5.

[95] J. C. Tannehill, D. A. Anderson, and R. H. Pletcher, Computational Fluid Mechanics and Heat Transfer, second ed., Series in Computational and Physical Processes in Mechanics and Thermal Sciences, Taylor and Francis, New York, 1997.

[96] G. I. Taylor, The instability of liquid surfaces when accelerated in a direction perpendicular to their planes, Proc. R. Soc. Lond. A Mat. 201 (1950), 192-196.

[97] G. Tryggvason, Numerical simulations of the Rayleigh-Taylor instability, J. Comput. Phys. 75 (1988), 253-282. 
[98] M. Vandenboomgaerde, S. Gauthier, and C. Mügler, Nonlinear regime of a multimode Richtmyer-Meshkov instability: a simplified perturbation theory, Phys. Fluids 14 (2002), $1111-1122$.

[99] M. Vandenboomgaerde, C. Mügler, and S. Gauthier, Impulsive model for the RichtmyerMeshkov instability, Phys. Rev. E 58 (1998), 1874-1882.

[100] M. Vetter and B. Sturtevant, Experiments on the Richtmyer-Meshkov instability of an air $/ S F_{6}$ interface, Shock Waves 4 (1995), 247-252.

[101] D. L. Youngs, Numerical simulation of turbulent mixing by Rayleigh-Taylor instability, Physica D 12 (1984), 32-44.

[102] _ Three-dimensional numerical simulations of turbulent mixing by Rayleigh-Taylor instability, Phys. Fluids A 3 (1991), 1312-1320.

[103] _ Numerical simulation of mixing by Rayleigh-Taylor and Richtmyer-Meshkov instabilities, Laser Part. Beams 12 (1994), 725-750.

[104] N. J. Zabusky, Vortex paradigm for accelerated inhomogeneous flows: Visiometrics for the Rayleigh-Taylor and Richtmyer-Meshkov environments, Ann. Rev. Fluid Mech. 31 (1999), 495-536.

[105] N. J. Zabusky, A. D. Kotelnikov, Y. Gulak, and G. Peng, Amplitude growth rate of a Richtmyer-Meshkov unstable two-dimensional interface to intermediate times, J. Fluid Mech. 475 (2003), 147-162.

[106] Q. Zhang, Analytical solution of Layzer-type approach to unstable interfacial fluid mixing, Phys. Rev. Lett. 81 (1998), 3391-3394.

[107] Q. Zhang and S. Sohn, Non-linear theory of unstable fluid mixing driven by shock wave, Phys. Fluids 9 (1997), 1106-1124.

[108] S. Zhang, N. J. Zabusky, G. Peng, and S. Gupta, Shock gaseous cylinder interactions: Dynamically validated initial conditions provide excellent agreement between experiments and numerical simulations to late-intermediate time, Phys. Fluids 16 (2004), 1203-1216. 